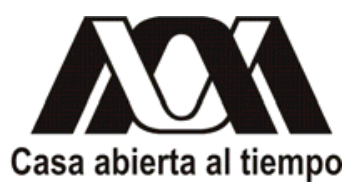

UNIVERSIDAD AUTÓNOMA METROPOLITANA UNIDAD IZTAPALAPA DIVISIÓN DE CIENCIAS BÁSICAS E INGENIERÍA

\title{
Estudio de la transferencia de energía, masa y cantidad de movimiento en sistemas fluido-medio poroso
}

TESIS QUE PRESENTA EL M.C. José Javier Valencia López

PARA OBTENER EL GRADO DE DOCTOR EN CIENCIAS

Asesor: Dr. J. Alberto Ochoa Tapia

Julio, 2005 


\section{Para:}

Yalí, Andrea y Arantxa 


\section{Agradecimientos:}

Al Dr. J. Alberto Ochoa T. por el insuperable apoyo como asesor en mis estudios de posgrado, pero principalmente por su amistad.

Al cuerpo de sinodales: Dra. Rosa María Velasco B.; Dr. Francisco Javier Sánchez B., Dr. Eduardo Ramos M. y Dr. Hugo Jiménez I., por los valiosos comentarios que ayudaron a enriquecer el presente trabajo.

Al Consejo Nacional de Ciencia y Tecnología (CONACyT) por el apoyo económico otorgado para la realización de mis estudios de doctorado. 


\section{Resumen.}

El transporte convectivo provocado por gradientes de temperatura y/o concentración frecuentemente se encuentra en problemas de la ingeniería química. En particular, la situación es importante cuando este tipo de transporte se presenta en cavidades, sean cerradas o abiertas. La mayor parte de los estudios de convección natural reportados son para sistemas de una fase o un medio multifásico homogéneo. Sin embargo, muchos de los problemas en ingeniería química se refieren al intercambio de masa, energía y cantidad de movimiento entre un fluido (región I) y un medio poroso (región II).

La complejidad de los modelos para este sistema aumenta, debido a que es necesario considerar las ecuaciones de transporte en cada región y las condiciones de frontera que las acoplan. Al respecto, la mayoría de los estudios realizados previamente se refieren a sistemas en los que existe sólo transporte bidimensional. Así, como la mayor parte de los estudios encontrados en la literatura se limitan a sistemas de una sola fase, hay también pocos estudios que se refieren a la validez de las condiciones de frontera que se han usado. De esta manera el desarrollo de modelos para el transporte entre un medio poroso y un fluido, en términos de ecuaciones diferenciales y condiciones de frontera, no es trivial.

Motivado en lo anterior, el presente trabajo tiene como objetivo el desarrollo, solución y pruebas numéricas de dos modelos para el proceso de convección natural en una cavidad ocupada parcialmente por un medio poroso en términos de ecuaciones de medio efectivo. En el análisis numérico se imponen gradientes de temperatura y concentración para analizar el efecto sobre los coeficientes de transferencia de los dos modelos mencionados y de la naturaleza bidimensional o tridimensional del transporte.

Los objetivos específicos del presente trabajo son:

1. El estudio del fenómeno de convección natural tridimensional en cavidades fluido-medio poroso.

2. El desarrollo de un modelo de difusión doble con transporte bidimensional, y 


\section{Resumen}

3. El desarrollo de la condición de salto en la interregión fluido-medio poroso para transferencia de masa.

La presentación del trabajo se estructura en cinco capítulos y tres apéndices. A continuación se da una breve descripción de cada uno de ellos.

En el Capítulo 1, se presenta una revisión bibliográfica de los diversos temas alrededor de este trabajo, considerando: flujo en cavidades, flujo en medios porosos, convección natural en sistemas con una región, en sistemas con dos regiones y flujo tridimensional. También se revisan trabajos enfocados al estudio de difusión doble y acoplamiento de ecuaciones en ambas regiones.

En el Capítulo 2, se establece el sistema físico y las simplificaciones impuestas para el análisis de la convección natural considerando un sistema en tres dimensiones; posteriormente se plantean las ecuaciones de transporte de energía, masa y cantidad de movimiento utilizadas para establecer dos modelos que consideran el transporte en cada una de las regiones. El primer modelo utiliza la ecuación de Darcy con la corrección de Brinkman, mientras que el segundo considera solamente la ecuación de Darcy para la transferencia de cantidad de movimiento en la región porosa, ocasionando que para la condición interregional se requiera la condición semi-empírica de Beavers y Joseph. Al final, se presentan en forma de resumen las ecuaciones que conforman cada uno de los dos modelos tridimensionales.

En el Capítulo 3, se presenta el esquema para la solución numérica de las ecuaciones diferenciales parciales (EDP) que conforman los modelos; esto incluye la discretización de las EDP y la descripción del esquema numérico.

En el Capítulo 4, se presentan las pruebas realizadas con el modelo computacional, ellas incluyen el análisis del efecto sobre la convección natural de los grupos adimensionales de Rayleigh $(R a)$, Darcy $(D a)$ y Lewis $(L e)$.

En el Capítulo 5, se presentan las conclusiones y perspectivas relacionadas al estudio presentado en los capítulos 3 y 4.

El desarrollo de las condiciones de salto para la transferencia de masa y las ecuaciones promedio utilizadas se presenta en los Apéndices A y B respectivamente, en el Apéndice C se reporta el desarrollo del modelo bidimensional para el proceso de convección natural ocasionada por gradientes simultáneos de masa y temperatura en una cavidad con un fluido y un medio poroso en la parte inferior, finalmente, en el Apéndice D se muestran las formas discretizadas en diferencias finitas para las derivadas involucradas en el modelo computacional. 
Como aportaciones importantes del trabajo se tienen:

- El desarrollo de un modelo que considera los efectos tridimensionales en la formulación vorticidad-vector potencial para el modelado de flujo convectivo en cavidades fluido-medio poroso, considerando la ley de Darcy o la corrección de Brinkman para representar el flujo en el medio poroso.

- Los resultados para la formulación tridimensional de los efectos de los números de Rayleigh, Prandtl y Lewis sobre el fenómeno de convección natural. Estos resultados se comparan con los obtenidos mediante un modelo que considera sólo efectos bidimensionales.

- El análisis de la ecuación de transferencia de masa (considerando difusión y adsorción) en la frontera entre un fluido homogéneo y un medio poroso, partiendo de las ecuaciones usualmente utilizadas. El desarrollo ha impuesto la condición de continuidad en la frontera para la concentración del soluto y se derivó una condición de salto para el flux que asegura el cumplimiento de la ecuación generalizada para el transporte de masa. 


\section{Índice.}

Resumen. I

Lista de Símbolos.

Lista de Figuras. $\quad$ XIV

Lista de Tablas. $\quad$ XVIII

Capítulo 1

Revisión Bibliográfica. $\quad 1$

1.1 Introducción. $\quad 1$

1.2 Flujo convectivo en cavidades. $\quad 2$

1.3 Flujo convectivo de calor en medios porosos.

1.4 Sistemas fluido medio poroso. 6

1.5 Difusión Doble. $\quad 7$

1.6 Condiciones en la interregión. $\quad 9$

Capítulo 2

Planteamiento del problema. 12

$\begin{array}{ll}2.1 \text { Sistema. } & 13\end{array}$

2.2 Ecuaciones del medio poroso. 13

$\begin{array}{lr}2.3 \text { Transferencia de calor. } & 15\end{array}$

2.3.1 Ecuación en la región fluida. $\quad 15$ 
2.3.2 Ecuación en la región porosa. 16

2.3.3 Ecuación en la interregión. 17

2.4 Transferencia de masa en el problema de difusión doble. 18

2.4.1 Ecuación en la región fluida. $\quad 18$

2.4.2 Ecuación de transferencia de masa en la región porosa. $\quad 19$

2.4.3 Condiciones de frontera y ecuación en la interregión para transferencia de masa. 19

2.5 Transferencia de cantidad de movimiento.

2.5.1 Ecuación para la transferencia de cantidad de movimiento en la región fluida. 21

2.5.2 Ecuación para la transferencia de cantidad de movimiento en la región porosa. 23

a) Ecuación de Darcy. 23

b) Extensiones a la ecuación de Darcy. 25

2.5.3 Formulación verticidad-vector potencial. 26

2.5.4 Condiciones de frontera y ecuación en la interregión para la transferencia de cantidad de movimiento. 28

a) Condición de Beavers y Joseph para la transferencia de cantidad de movimiento en la interregión.

2.6 Resumen de ecuaciones para los dos modelos.

\section{Capítulo 3}

Procedimiento numérico.

3.1 Ecuaciones Elípticas. $\quad 37$

3.2 Ecuaciones Parabólicas. $\quad 37$

3.3 Diferencias finitas. $\quad 39$

3.3.1 Discretización de la ecuación de energía. 40

3.3.2 Ecuación de transferencia de masa. 52

3.4 Condiciones de frontera e interregionales. 55

$\begin{array}{ll}3.5 \text { Estabilidad y convergencia. } & 61\end{array}$ 


\section{Capítulo 4}

Pruebas e interpretación de resultados. $\quad 64$

4.1 Condiciones de frontera. $\quad 65$

4.2 Parámetros numéricos. $\quad 67$

4.3 Efecto de los Números de Rayleigh y Darcy.

$\begin{array}{lr}\text { 4.3.1 Número de Nusselt. } & 79\end{array}$

4.4 Variación de la Relación de Conductividades. 102

4.5 Variación de la interregión. $\quad 104$

4.6 Efecto del término convectivo. 108

4.7 Pruebas considerando efectos tridimensionales. 112

4.8 Pruebas al modelo de difusión doble. 115

4.8.1 Variación de la interregión. $\quad 115$

4.8.2 Variación del Número de Flotación. 118

Capítulo 5

Conclusiones.

5.1 Conclusiones. $\quad 122$

5.2 Perspectivas. 124

$\begin{array}{ll}\text { Bibliografía } & 125\end{array}$

Apéndice A

Condición de salto para el transporte de masa en la frontera de un medio poroso y un fluido homogéneo.

A.1 Ecuaciones puntuales.

A.2 Volumen Promedio. 
Apéndice B

Ecuación de transferencia de masa en el medio poroso.

B.1 Transporte convectivo.

B.2 Transporte difusivo.

B.3 Problema de cerradura.

\section{Apéndice $\mathrm{C}$}

Desarrollo del modelo en dos dimensiones.

C.1 Sistema.

C.2 Formulación matemática.

C.3 Formulación vorticidad-función de corriente.

184

C.4 Ecuaciones adimensionales.

C.5 Condiciones en las paredes del sistema.

C.6 Método numérico.

\section{Apéndice D}

Discretización en diferencias finitas con espaciamiento variable.

D.1 Diferencias centradas.

D.1.1 Primer derivada.

D.1.2 Segunda derivada.

D.2 Diferencias hacia adelante.

D.2.1 Primer derivada.

D.1.2 Segunda derivada.

D.3 Diferencias hacia atrás.

D.3.1 Primer derivada. 


\section{Lista de Símbolos.}

$$
\begin{array}{ll}
a_{v} & =\frac{A_{\beta \sigma}}{V} \text {; Área interfacial por unidad de volumen }\left(\mathrm{m}^{-1}\right) . \\
A_{\beta \sigma} & =A_{\sigma \beta} \text {; Área de la interface } \beta \sigma \text { dentro de la región macroscópica }\left(\mathrm{m}^{2}\right) . \\
A_{\beta e} & \text { Área de entradas y salidas de la fase } \beta \text { en la frontera de la región } \\
& \text { macroscópica }\left(m^{2}\right) .
\end{array}
$$

$A_{\omega \eta} \quad=A_{\eta \omega}$; Área de la superficie de separación contenida en el volumen $V_{\infty}\left(m^{2}\right)$.

C Concentración dimensional $\left(\mathrm{mol} / \mathrm{m}^{3}\right)$.

$C_{i} \quad$ Concentración puntual en la fase $i\left(\mathrm{~mol} / \mathrm{m}^{3}\right)$.

$C_{A s} \quad$ Concentración superficial de $A\left(\mathrm{~mol} / \mathrm{m}^{3}\right)$.

$\tilde{C}_{A} \quad=C_{A}-\left\langle C_{A}\right\rangle^{\beta}$; Desviación espacial de la concentración en la fase $\beta\left(\mathrm{mol} / \mathrm{m}^{3}\right)$.

$\left\langle C_{A}\right\rangle^{\beta} \quad \varepsilon_{\beta}\left\langle C_{A}\right\rangle$; Promedio intrínseco de la concentración en la fase $\beta\left(\mathrm{mol} / \mathrm{m}^{3}\right)$.

$\left\langle C_{A}\right\rangle_{\eta} \quad$ Promedio superficial de la concentración en la fase $\beta$ que es determinada por las ecuaciones que sean válidas en la región homogénea $\eta\left(\mathrm{mol} / \mathrm{m}^{3}\right)$.

$\left\langle C_{A}\right\rangle_{\omega} \quad$ Promedio superficial de la concentración en la fase $\beta$ que es determinada por las ecuaciones que sean válidas en la región homogénea $\omega\left(\mathrm{mol} / \mathrm{m}^{3}\right)$.

$\left\langle C_{A}\right\rangle_{\beta \sigma} \quad$ Concentración promedio interfacial $\left(\mathrm{mol} / \mathrm{m}^{3}\right)$.

$\left(\rho C_{p}\right)_{\beta} \quad$ Capacidad calorífica volumétrica de la fase $\beta\left(\mathrm{J} / \mathrm{m}^{3} \mathrm{~K}\right)$.

$\left(\rho C_{p}\right)_{\sigma} \quad$ Capacidad calorífica volumétrica de la fase $\sigma\left(\mathrm{J} / \mathrm{m}^{3} \mathrm{~K}\right)$.

$\left(\langle\rho\rangle C_{p}\right)_{s}$ Exceso superficial de la capacidad calorífica de acumulación $\left(\mathrm{J} / \mathrm{m}^{3} \mathrm{~K}\right)$.

$\left(\rho C_{p}\right)_{s} \quad$ Exceso superficial de la capacidad calorífica $\left(\mathrm{J} / \mathrm{m}^{3} \mathrm{~K}\right)$.

$C_{p} \quad=\frac{\left[\varepsilon_{\beta}\left(\rho C_{p}\right)_{\beta}+\varepsilon_{\sigma}\left(\rho C_{p}\right)_{\sigma}\right]}{\langle\rho\rangle}$; Capacidad calorífica volumétrica ponderada por la fracción másica $(\mathrm{J} / \mathrm{Kg} \mathrm{K})$. 
$C_{p \omega} \quad$ Capacidad calorífica volumétrica ponderada por la fracción másica para la región $\omega(\mathrm{J} / \mathrm{Kg} \mathrm{K})$.

$C_{p \eta} \quad$ Capacidad calorífica volumétrica ponderada por la fracción másica para la región $\eta(\mathrm{J} / \mathrm{Kg} \mathrm{K})$.

D Diámetro del volumen promedio teniendo la forma de un disco $(m)$.

$\mathbf{D}_{\text {eff }} \quad$ Tensor difusividad $\left(\mathrm{m}^{2} / \mathrm{s}\right)$.

$\mathbf{D}_{D} \quad$ Tensor dispersivo $\left(\mathrm{m}^{2} / \mathrm{s}\right)$.

D* Tensor dispersivo total $\left(\mathrm{m}^{2} / \mathrm{s}\right)$.

Da $\quad=\frac{\mathbf{K}}{L_{x}^{2}}$; Número de Darcy.

$D_{\beta} \quad$ Representa el coeficiente de difusión de la mezcla en la fase $\beta\left(\mathrm{m}^{2} / \mathrm{s}\right)$.

$g^{\prime} \quad$ Aceleración de la gravedad $\left(\mathrm{m} / \mathrm{s}^{2}\right)$.

g Tensor gravedad $\left(\mathrm{m} / \mathrm{s}^{2}\right)$.

$K \quad$ Permeabilidad del medio poroso $\left(\mathrm{m}^{2}\right)$.

$K_{e q} \quad$ Coeficiente lineal de distribución de equilibrio $(m)$.

$k_{\gamma} \quad$ Conductividad térmica $(\mathrm{J} / \mathrm{m} \mathrm{s} \mathrm{K})$.

$K_{\omega}=\frac{\left(\alpha_{\beta}\right)_{\omega}}{\left(\alpha_{\beta}\right)_{\eta}} ;$ Relación de difusividades térmicas.

Le $\quad L e_{\eta}=\frac{\left(\alpha_{\beta}\right)_{\eta}}{\left(\mathrm{D}_{\beta}\right)_{\eta}}, L e_{\omega}=\frac{\left(\alpha_{\beta}\right)_{\eta}}{D_{\text {eff }}}$; Número de Lewis .

$L_{\psi} \quad$ Longitud característica asociada con la función genérica $\psi_{\beta}(m)$.

$L_{\psi 1} \quad$ Longitud característica asociada con el gradiente de la función genérica $\psi_{\beta}$ $(m)$.

$V_{\beta(\mathbf{x})} \quad$ Volumen de la fase $\beta$ contenido dentro del volumen promedio $\left(\mathrm{m}^{3}\right)$.

C Curva cerrada situada en la superficie divisoria de regiones.

$\sigma \quad$ Longitud de arco a lo largo de la curva C $(m)$.

$\delta \quad$ Grosor de la región de salto $(m)$.

$N \quad$ Relación de flotación.

$\mathrm{Nu} \quad$ Número de Nusselt.

$\mathbf{n}_{\beta \sigma} \quad=-\mathbf{n}_{\sigma \beta}$; Vector normal unitario en la dirección de la fase $\beta$ a la fase $\sigma$.

$\mathbf{n}_{\sigma \beta} \quad$ Vector normal unitario en la dirección de la fase $\beta$ a la fase $\sigma$.

$\mathbf{n}_{\omega \eta} \quad=-\mathbf{n}_{\eta \omega}$; Vector normal unitario dirigido de la región $\omega$ hacia la región $\eta$. 
$\mathbf{n}_{s} \quad$ Vector normal unitario tangencial a la superficie divisoria y normal a la curva C.

$\mathbf{P}=\mathbf{I}-\mathbf{n}_{\omega \eta} \mathbf{n}_{\omega \eta} ;$ Tensor proyección.

$\operatorname{Pr} \quad=\frac{\mu_{\beta}}{\rho_{\beta}\left(\alpha_{\beta}\right)_{\eta}} ;$ Número de Prandtl.

$p_{\beta} \quad$ Presión en la fase $\beta(P a)$.

$p_{\gamma}^{\prime} \quad$ Presión dimensional en la región fluida.

$R a_{\theta} \quad=\frac{g \beta_{\theta} \Delta T L_{x}^{3}}{\left(\alpha_{\beta}\right)_{\eta} v_{\beta}} ;$ Número de Rayleigh para la ecuación de energía.

$R a_{\phi} \quad=\frac{g \beta_{\phi} \Delta C_{A} L_{x}^{3}}{\left(D_{\beta}\right)_{\eta} v_{\beta}}$; Número de Rayleigh para la ecuación de transferencia de masa.

Sh Número de Sherwood.

$t \quad$ Tiempo $(s)$.

$T_{\beta} \quad$ Temperatura en la fase $\beta(K)$.

$\left\langle T_{\beta}\right\rangle \quad$ Promedio superficial de la temperatura en la fase $\beta(K)$.

$\left\langle T_{\beta}\right\rangle^{\beta} \quad=\varepsilon_{\beta}\left\langle T_{\beta}\right\rangle$; Promedio intrínseco de la temperatura en la fase $\beta(K)$.

$\widetilde{T}_{\beta} \quad=T_{\beta}-\left\langle T_{\beta}\right\rangle^{\beta}$; Desviación espacial para la temperatura en la fase $\beta(K)$.

$T_{\sigma} \quad$ Temperatura en la fase $\sigma(K)$.

$\left\langle T_{\sigma}\right\rangle \quad$ Promedio superficial de la temperatura en la fase $\sigma(K)$.

$\left\langle T_{\sigma}\right\rangle^{\sigma} \quad=\varepsilon_{\sigma}\left\langle T_{\sigma}\right\rangle$; Promedio intrínseco de la temperatura en la fase $\sigma(K)$.

$\tilde{T}_{\sigma} \quad=T_{\sigma}-\left\langle T_{\sigma}\right\rangle^{\sigma}$; Desviación espacial para la temperatura en la fase $\sigma(K)$.

$\langle T\rangle_{\omega} \quad$ Promedio espacial de la temperatura determinado por la ecuación que es válida en la parte homogénea de la región $\omega(K)$.

$\langle T\rangle_{\eta} \quad$ Promedio espacial de la temperatura determinado por la ecuación que es válida en la parte homogénea de la región $\eta(K)$.

$\mathbf{v}_{\beta} \quad$ Velocidad en la fase $\beta(\mathrm{m} / \mathrm{s})$.

$\left\langle\mathbf{v}_{\beta}\right\rangle \quad$ Promedio superficial de la velocidad en la fase $\beta(\mathrm{m} / \mathrm{s})$.

$\left\langle\mathbf{v}_{\beta}\right\rangle^{\beta} \quad=\varepsilon_{\beta}\left\langle\mathbf{v}_{\beta}\right\rangle$; Promedio superficial de la velocidad en la fase $\beta(\mathrm{m} / \mathrm{s})$.

$\widetilde{\mathbf{v}}_{\beta} \quad=\mathbf{v}_{\beta}-\left\langle\mathbf{v}_{\beta}\right\rangle^{\beta}$; Desviación espacial de la velocidad para la fase $\beta(\mathrm{m} / \mathrm{s})$. 


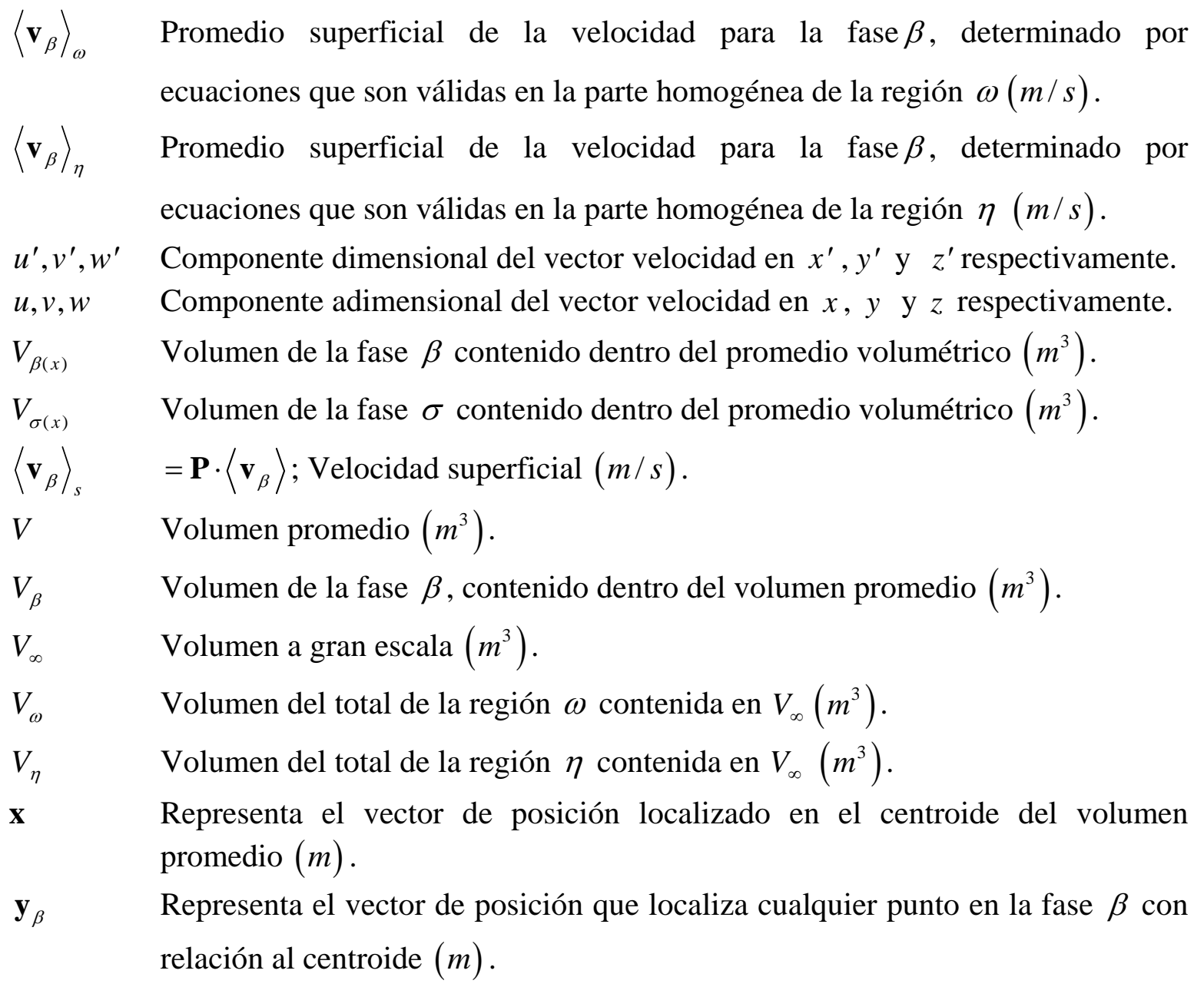

\section{Símbolos griegos:}

$\begin{array}{ll}\alpha_{\gamma} & \text { Difusividad térmica de la fase fluida }\left(\mathrm{m}^{2} / \mathrm{s}\right) . \\ \beta_{\theta} & \text { Coeficiente de expansión térmica de la fase fluida }\left(\mathrm{mol} / \mathrm{m}^{3}\right)^{-1} . \\ \beta_{\phi} & \text { Coeficiente de expansión másica de la fase fluida }\left(\mathrm{mol} / \mathrm{m}^{3}\right)^{-1} . \\ \gamma & \text { Fase fluida. } \\ \eta & \text { Región fluida. } \\ \varepsilon_{\beta} & =\frac{V_{\beta}}{V} \text {; Porosidad o fracción volumen de la fase } \beta . \\ \varepsilon_{\sigma} & =\frac{V_{\sigma}}{V} \text {; Porosidad o fracción volumen de la fase } \sigma . \\ \xi_{\beta} & \text { Cualquier propiedad vectorial. } \\ \theta & \text { Temperatura adimensional. } \\ \mu_{\beta} & \text { Viscosidad de la fase } \beta(\mathrm{Kg} / \mathrm{m} / \mathrm{s}) .\end{array}$




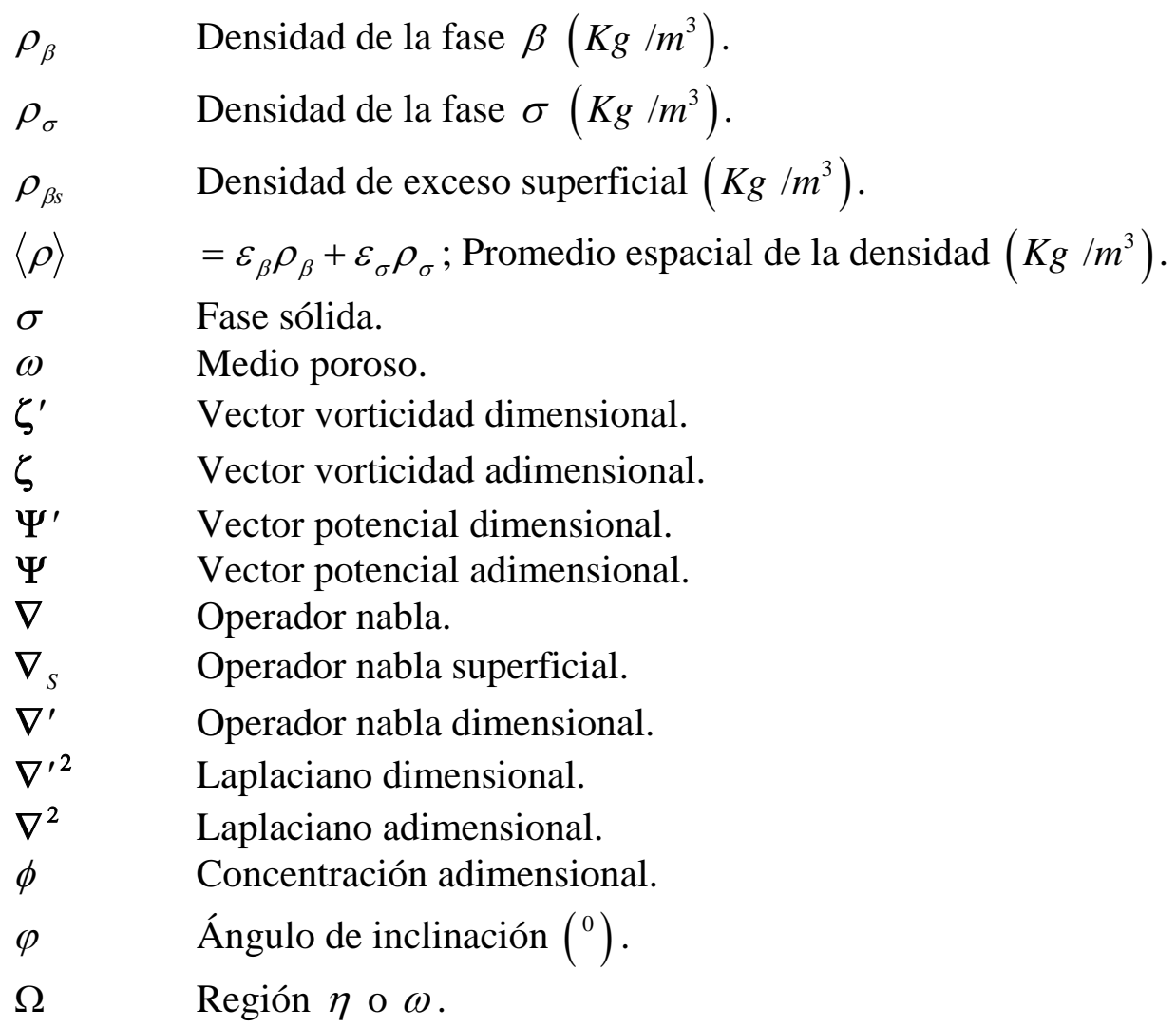

\section{Subíndices:}

$i \quad$ Indica fase $i, i=\beta, \sigma$.

$S \quad$ Posición de la interregión fluido-medio poroso.

$\omega \quad$ Región fluida homogénea.

$\eta \quad$ Región porosa homogénea.

$\sigma \quad$ Fase sólida.

$\beta \quad$ Fase fluida. 


\section{Lista de Figuras.}

Figura 2.1.- $\quad$ Cavidad utilizada para el desarrollo de las ecuaciones. $\quad 14$

Figura 2.2.- $\quad$ Condición interregional de Beavers y Joseph. 31

Figura 3.1.- Asignación del espaciamiento variable y celda computacional para el sistema tridimensional. $\quad 41$

Figura 3.2.- $\quad$ Secuencia de evaluación en el método ADI. 45

Figura 3.3.- $\quad$ Incrementos variables para la discretización de las ecuaciones. 47

Figura 3.4.- Secuencia para la evaluación de las condiciones frontera e interregional. 56

Figura 3.5.- $\quad$ Secuencia de evaluación de las ecuaciones.. $\quad 62$

Figura 4.1.- $\quad$ Condiciones de frontera para la ecuación de energía. 66

Figura 4.2.- Superficies y mapas de contorno para las pruebas del modelo tridimensional.

Figura 4.3.- Mapas de contorno de temperatura modelos tridimensionales: ByJ; Brinkman, $\mathrm{Xs}=0.5$.

Figura 4.4.- Comparación de los perfiles de temperatura a $Y=0.25$ y 0.5, Modelos en 3D, Rayleigh $10^{6}$, Darcy $10^{-3}$.

Figura 4.5.- $\quad$ Comparación de los perfiles de temperatura a $Y=0.25$, Modelos en 2D y 3D, Rayleigh $10^{6}$, Darcy $10^{-3}$.

Figura 4.6.- $\quad$ Superficies, comparación de los perfiles de temperatura para los modelos de a) Brinkman y b) B y J.

Figura 4.7.- Mapas del contorno para el Número de Nusselt: modelos tridimensionales: - - B y J; $\square$ Brinkman, $X_{\mathrm{S}}=0.5$.

Figura 4.8.- Comparación del número de Nusselt local en la pared fría, obtenida mediante a)Brinkman y b) B y J: $R a=10^{6}, D a=10^{-3}$, parámetro de Beavers y Joseph =1.0 
Figura 4.9.- Comparación del número de Nusselt local en la pared fría a) Brinkman y b)B y J: $R a=10^{6}, D a=10^{-3}$, Parámetros de Beavers y Joseph $=1.0$

Figura 4.10.- Superficie y mapa de contorno para la componente $\mathrm{z}$ de la velocidad $(w)$ modelos tridimensionales: - -B y J; Brinkman; $X \mathrm{~s}=0.5, R c=1.0$

Figura 4.11.- Mapas de contorno para el potencial $\left(\Psi_{z}\right)$ modelos tridimensionales: - - Modelo de Beavers y Joseph; Corrección de Brinkman a la ecuación de Darcy; Xs=0.5, $R c=1.0$

Figura 4.12.- Comparación de los perfiles del potencial $\left(\Psi_{z}\right) Y=0.25$ y 0.5 , Modelos en 3D, $R a=10^{6}, \mathrm{Da}=10^{-3}$

Figura 4.13.- Comparación de los perfiles del potencial $\left(\Psi_{z}\right) Y=0.25$, Modelos en 2 y $3 \mathrm{D}, R a=10^{6}, \mathrm{Da}=10^{-3}$

Figura 4.14.- Comparación de los perfiles del potencial $\left(\Psi_{z}\right) Y=0.25$, Modelos 3D, Darcy $=10^{-3}$

Figura 4.15.- Superficies, para el potencial $\left(\Psi_{z}\right)$ modelos tridimensionales a) Brinkman b) Beavers y Joseph

Figura 4.16.- Mapas de contorno para la componente $\mathrm{z}$ de la velocidad $(w)$ modelos tridimensionales: - - B y J.; Brinkman, $\mathrm{Xs}=0.5$, $R C=1.0$

Figura 4.17.- Comparación de los perfiles de la componente $\mathrm{Z}$ de la velocidad (w) a $Y=0.25, Y=0.5$ Modelos en 2 y $3 \mathrm{D}, R a=10^{6}, \mathrm{Da}=10^{-3}$.

Figura 4.18.- Comparación de los perfiles de la componente $\mathrm{Z}$ de la velocidad $(w)$ a $Y=0.25, Y=0.5$, Modelos en 3D, $R a=10^{6}, \mathrm{Da}=10^{-3}$.

Figura 4.19.- Comparación de los perfiles de la componente $\mathrm{Z}$ de la velocidad (w) a $Y=0.5$, Modelos 3D, $D a=10^{-4}$.

Figura 4.20.- Superficies para la componente $\mathrm{Z}$ del vector velocidad $(w)$, variación del $\mathrm{Ra}$ y $\mathrm{Da}$, modelos tridimensionales

Figura 4.21.- Isotermas para analizar la relación de conductividades, modelos tridimensionales: - - B y J; Brinkman. 
Figura 4.22.- Posición de la interregión para las pruebas en tres dimensiones.

Figura 4.23.- Variación de la interregión: a)Isotermas b)Velocidad c) líneas de corriente d) Número de Nusselt, modelos tridimensionales: - - B y $\mathrm{J} ; \quad$ Brinkman, $R c=1.0$

Figura 4.24.- Mapas de contorno para la componente $\mathrm{z}$ de la velocidad $(w)$ modelos tridimensionales: - - Jiménez-Islas, Brinkman, Xs=0.5, $R C=1.0$

Figura 4.25.- Término convectivo: mapas de contorno para la componente Z de la velocidad $(w)$ modelos tridimensionales: - - B y J;

Brinkman.

Figura 4.26.- Mapas de contorno para la componente $Z$ de la velocidad $(w)$ e isotermas Con término convectivo : - - Sin término convectivo: Modelo de Brinkman.

Figura 4.27.- Influencia del término convectivo: Componente $\mathrm{Z}$ de la velocidad (w) .

Figura 4.28 Condiciones de frontera para analizar efectos tridimensionales.

Figura 4.29.- Mapas de contorno para la temperatura y las componente $X$ y $Y$ del potencial: Presente trabajo (Brinkman), - - Jiménez (1999).

Figura 4.30 Condición de frontera para la variación de la interregión, modelo bidimensional.

Figura 4.31 Mapas de contorno para: a) Isotermas b) líneas de concentración

c) líneas de corriente d) velocidad, prueba de variación de la interregión.

Figura 4.32.- Condiciones de frontera para el análisis de fuerzas de flotación.

Figura 4.33 Mapas de contorno para a) Función de corriente, b) Isotermas y c) Líneas de concentración, flujo inverso, $\mathrm{Ra}=10^{4}, \mathrm{Pr}=10.0, \mathrm{X}_{\mathrm{I}}$ $=0.0$

Figura 4.34 Superficies para la función de corriente, prueba para analizar el flujo inverso.

Figura A-1 Sistema considerado.

Figura A-2. Posible ubicación del volumen promedio que muestra que la fracción volumétrica es función de la posición del volumen promedio.

Figura A-3. Volumen promedio y vectores de posición asociados para el sistema fluido-medio poroso. 
Figura A-4. Continuidad del campo intrínseco y la aproximación en la solución de las ecuaciones válidas en la parte homogénea de las regiones.

Figura A-5. Volumen promedio a gran escala en la interregión fluido - medio poroso.

Figura B-1. Transporte convectivo en un sistema poroso (Fluido-sólido).

Figura C-1. Sistema en dos dimensiones con inclinación $\varphi$.

Figura C.2. Notación para condiciones de frontera en la transferencia de energía.

Figura C-3 Espaciamiento en la interregión para la discretización de la condición de Beavers y Joseph

Figura D.1.- Celda computacional utilizada para la discretización de las ecuaciones diferenciales en diferencias finitas. 


\section{Lista de Tablas.}

Tabla 3.1 Coeficientes utilizados para el método del falso estado transitorio. 39

Tabla 3.2 Coeficientes utilizados en la ecuación de energía. 40

Tabla 4.1 Niveles de los parámetros analizados en el caso tridimensional. $\quad 65$

Tabla 4.2 Valores para el método de falso estado transitorio en ambas $\begin{array}{ll}\text { regiones. } & 67\end{array}$

Tabla 4.3 Iteraciones y tiempos de cómputo para diferentes $R a, D a$ y tamaño $\begin{array}{ll}\text { de malla. } & 69\end{array}$

Tabla 4.4 Efecto del número de Rayleigh, Darcy y tamaño de la red sobre el número de Nusselt promedio de las paredes fría y caliente obtenidos con los modelos de Darcy y con la corrección de Brinkman.

Tabla C-1. Variables adimensionales. 186

Tabla C-2. Números adimensionales. $\quad 186$

Tabla C-3 Parámetros utilizados en la ecuación de energía. 193 


\section{Capítulo 1}

\section{Revisión Bibliográfica.}

\subsection{Introducción.}

El flujo por convección natural se presenta cuando se generan fuerzas de flotación debido a un gradiente de temperatura y/o concentración en una cavidad, (Leonardi, 1984). La importancia que ha adquirido este fenómeno en años recientes ha obligado a que los avances sean específicos para cada problema particular tanto en el aspecto físico, geométrico, métodos de solución, y condiciones de frontera entre otros aspectos.

Considerando que uno de los objetivos del presente trabajo es desarrollar un modelo tridimensional para la convección natural en un sistema fluido-medio poroso, en el presente capítulo se presenta una revisión bibliográfica, cuyo objetivo es analizar las alternativas que se han utilizado para plantear y resolver dicho problema, ya sea bidimensional o tridimensional; considerando una o dos regiones, así como las diferentes alternativas numéricas para su solución. Para ello se presenta una revisión bibliográfica que se ha dividido en los siguientes temas:

1) Flujo convectivo en cavidades: Se muestran los trabajos publicados para analizar la convección natural en sistemas considerando dos o tres dimensiones, en cavidades que contienen un fluido.

2) Flujos convectivo en medios porosos: Se presentan los trabajos que consideran un medio multifásico homogéneo en el fenómeno de convección natural.

3) Flujo en cavidades fluido-medio poroso: Considera los trabajos que resuelven el problema de convección libre en cavidades formadas por un fluido y un medio poroso, la mayoría de lo trabajos reportados cubren solo cavidades bidimensionales. 
4) Fenómeno de difusión doble: La revisión abarca los trabajos bidimensionales realizados al fenómeno de convección natural provocado por gradientes simultáneos de temperatura y de concentración en cavidades con un fluido o un sistema fluidomedio poroso.

5) Condiciones para acoplar las ecuaciones en sistemas multirregionales: Se presentan los trabajos que muestran las diferentes formas que se han utilizado para acoplar las ecuaciones de transporte (básicamente la ecuación de transferencia de cantidad de movimiento) en un sistema fluido-medio poroso.

En cada uno de los temas en que se ha dividido la revisión se presenta una pequeña introducción para analizar su aplicación y posteriormente una revisión de los trabajos publicados, finalmente como conclusión del capítulo se presentará un resumen para mostrar los aspectos que se consideran en el problema que se aborda en este trabajo.

\subsection{Flujo convectivo en cavidades.}

El análisis de flujo convectivo en cavidades tiene muchas aplicaciones tecnológicas en ingeniería térmica, tales como el enfriamiento de mecanismos electrónicos, aislamiento térmico de edificios, sistemas de almacenamiento de energía, colectores de energía solar, seguridad en reactores nucleares y compartimientos de seguridad para incendios. Otras aplicaciones están relacionadas a ventanas de doble cristal para aislamiento térmico, cavidades de aislamiento edificios (Ciofalo y Karayiannis, 1991; Kelkar y Patankar, 1990). El problema es de igual forma interesante para los geofísicos en simulación de las condiciones reales de movimiento de fluido como resultado de las fuerzas de flotación por gradientes de temperatura dentro de los pozos.

Batchelor (1954) fue uno de los primeros autores en establecer la formulación matemática para flujo en cavidades, por limitaciones de cómputo, en su trabajo sólo se proporcionaron soluciones aproximadas. Poots (1962) realizó cálculos manuales y mostró las primeras gráficas para isotermas y líneas de corriente dentro de una cavidad. Hellums y Churchill (1962) desarrollaron cálculos numéricos y compararon sus resultados con datos experimentales obtenidos anteriormente por Martini y Churchill (1960). Posteriormente Ostrach (1967) realizó cálculos para valores altos de Número de Rayleigh, en ese mismo año Samuels y Churchill (1967) usaron el método de diferencias finitas para calcular la inestabilidad hidrodinámica debido a la convección natural en una región cerrada rectangular horizontal. En estos primeros trabajos la convección natural es descrita por las ecuaciones de conservación de masa, energía y cantidad de movimiento. Los cálculos que se presentan suponen que el movimiento del fluido es bidimensional y se utilizan ecuaciones basadas en la formulación función de corriente-vorticidad. 
Posteriormente, Leonardi en 1984 presenta un trabajo que considera los efectos tridimensionales, el autor realizó un estudio numérico de los efectos que se tienen en la convección natural al variar las propiedades de un fluido almacenado en un contenedor rectangular; Las pruebas numéricas se realizaron para aire y agua como fluidos y los efectos que se analizaron están relacionados con la variación de densidad, presión, viscosidad y conductividad térmica. Los resultados muestran que todas estas propiedades tienen efectos importantes sobre el flujo y perfiles de temperatura para cavidades que contienen aire, mientras que para aquellas que contienen agua, sólo la densidad y viscosidad tienen un efecto considerable. Continuando con los estudios en tres dimensiones, Le Peutrec y Lauriat (1990) consideraron los efectos de la transferencia de calor por convección natural en las paredes laterales dentro de cavidades, los autores investigaron los efectos de las pérdidas de calor por conducción en las paredes laterales, los resultados reportados por estos permiten evaluaciones cuantitativas de los efectos de las pérdidas de calor a los alrededores.

Aunque desde 1980 se tenían soluciones numéricas para cavidades tridimensionales, los estudios en dos dimensiones continuaron presentándose, aunque ahora los estudios se realizaron para aspectos específicos del fenómeno, así pues, Barakos y col. (1994) al igual que Hanjalic (1994) realizaron estudios para analizar la transición de flujo laminar a turbulento en una cavidad bidimensional, mientras que Bian y col. (1994) y Martins-Costa y Saldanha de Gama. (1994) analizan los efectos de la convección natural en la cercanía de las paredes, Poujol y col. (2000) presentan un estudio experimental para la convección natural en una cavidad cúbica, los autores enfocan su estudio a fluidos con alto número de Prandtl (230), los resultados experimentales fueron comparados con la solución numérica de las ecuaciones gobernantes en un sistema bidimensional, Prud'homme y col. (2003) realizaron estudios de estabilidad numérica para una cavidad vertical bidimensional sujeto a diferencias de temperatura en las cuatro paredes, estableciendo los rangos del número de Prandtl para los cuales se presenta estabilidad; Dong. y Li (2004) analizan el método vorticidad-función de corriente para analizar la transferencia de calor en el proceso de convección natural aplicado a geometrías complejas.

Respecto a los estudios numéricos para el análisis de la convección en cavidades, se puede observar que se enfocan al análisis de varios aspectos del flujo confinado, dentro de éstos se encuentran las interacciones entre las partes cercanas a las fronteras con las partes medias (Aziz y Hellums, 1985; Ostrach, 1988), los efectos geométricos de las cavidades como el relación de aspecto y la inclinación (Bennacer y Gobin ,1996; Catton, 1978; Hoogendoorn, 1986; Fusegi y Hyun, 1994), mejoras a los métodos numéricos basados en la discretización en diferencias finitas (Ding y col., 2004) y consideraciones a modelos en tres dimensiones para bajos números de Prandtl (Wakitani, 2001).

Los avances en el campo computacional han contribuido a que en años recientes los estudios numéricos se enfoquen a la utilización de métodos combinados o mejoras a los 
utilizados tradicionalmente. Como ejemplos se tiene que Peng y col. (2003), utilizan un método al que denominan "modelo térmico de Lattice Boltzmann" para simular la convección natural en una cavidad tridimensional, mientras que Colomer y col. (2004), utilizan el "método de ordenadas discretas" para encontrar la solución del mismo problema. Por otra parte Ding y col. (2004) utilizan un método combinado de expansión en series de Taylor con mínimos cuadrados ponderados para analizar el flujo convectivo en una cavidad bidimensional. Adicionalmente Wakashima y Saitho (2004) utilizan un método tiempoespacio de alto orden para analizar los efectos del Número de Rayleigh en una cavidad tridimensional conteniendo aire.

La revisión realizada a flujos en cavidades muestra que se cuenta con una solución del problema general, esta solución se ha incluido en paquetes de cómputo comerciales de dinámica de fluidos, de igual forma, se han publicado textos con revisiones de los fundamentos de flujo convectivo en cavidades (Kaviany, 1991; Nield y Bejan, 1992), sin embargo, la vigencia del problema es evidente con la publicación de estudios recientes, estos avances se enfocan a reducir el tiempo de cómputo, a explorar nuevos métodos de solución o analizar detalles de la transferencia en las proximidades de las paredes.

\subsection{Flujo convectivo de calor en medios porosos.}

La transferencia de calor por convección en un medio poroso saturado con un fluido, ha adquirido una atención considerable en las décadas recientes debido a su relevancia en una amplia gama de aplicaciones, tales como: Diseño de aislamientos térmicos, almacenamiento y preservación de granos y cereales (Jiménez-Islas y col. 2004; Jiménez Islas, 1999) y la explotación eficiente de yacimientos petrolíferos. De igual forma, muchas operaciones industriales en las áreas de ingeniería química, bioquímica y metalúrgica involucran el paso de un fluido a través de un lecho empacado con partículas sólidas, lo anterior para obtener áreas de contacto interfacial fluido-sólido o bien un mejor mezclado de fluido. Algunos ejemplos típicos de aplicaciones que involucran a tales sistemas incluyen reacciones heterogéneas con catalizador y cromatográficas, sistemas de filtración, torres empacadas de adsorción y destilación, filtros empacados, migración de desechos radiactivos a través del suelo entre otras (Greenkorn, 1983; Nield y Bejan, 1992).

En 1984, Prasad y Kulacki (a,b) presentaron uno de los primeros trabajos que consideran la geometría y los efectos básicos en el fenómeno de convección natural en medios porosos, los estudios se realizaron en una cavidad bidimensional conteniendo medio poroso inmerso en un fluido, los autores consideraron una cavidad rectangular sometida a un gradiente de temperatura en sus paredes laterales, así como el efecto de una fuente calorífica en una pared lateral. Posteriormente Prasad (1987) analiza el efecto de la generación volumétrica de calor para un sistema con las mismas características físicas utilizadas en sus 
trabajos de 1984. En todas las publicaciones anteriores la solución numérica del problema se obtiene mediante el método del volumen finito de control (Patankar, 1980), los autores reportan los efectos del número de Rayleigh para medio poroso y del aspecto geométrico sobre patrones de flujo, las isotermas, el número de Nusselt promedio, así como un análisis de la generación del flujo multicelular.

La mayoría de los estudios efectuados para analizar el fenómeno de convección natural en un medio poroso, toman como ecuación de transferencia de cantidad de movimiento la Ley de Darcy (Whitaker, 1986). No obstante, se reconoce que los efectos no Darcianos son significativos en materiales de alta porosidad y permeabilidad (Lauriat, 1989). Existe un número considerable de estudios que han considerado estos efectos, entre ellos se pueden mencionar los trabajos reportados por Hsu y Cheng (1985) y Kim y Vafai (1989), en ambos casos se estudiaron los efectos de frontera basados en la corrección de Brinkman a la ecuación de Darcy. Por otro lado Bejan y Poulikakos (1984) y Plumb y Huenefeld (1981), han utilizado la corrección de Forschheimer a la ecuación de Darcy para incluir los efectos inerciales.

Varias aplicaciones relacionadas con el medio poroso requieren de un análisis detallado de la transferencia de calor por convección en diferentes geometrías (Prasad y Chui, 1989; Jiménez-Islas y col., 1999), orientaciones y configuraciones (Prasad y col., 1985; Dong y Li, 2004).

De igual forma, la aplicación de la convección en medios porosos asociado con tejidos biológicos ha mostrado gran interés, como ejemplo se tienen los trabajos presentados por: Dash y col. (1996); Preziosi y Farina (2002); Shih y col. (2002); Nield y col. (2004); Kuznetsov y Jiang (2001) y Kuznetsov y Avramenko (2002). Una revisión sobre los trabajos que abarcan este tema la realizaron Khaled y Vafai (2003), en éste se realiza una división de las aplicaciones de la convección en tejidos biológicos.

La dependencia con el tiempo en el proceso de convección natural en una cavidad bidimensional fue estudiada por Saeid y Pop (2004), los autores reportan el tiempo para alcanzar el estado estacionario en función del Número de Rayleigh, la cavidad analizada considera el gradiente de temperatura en las paredes verticales. Por otra parte, Tan y col. (2003) presentaron un estudio similar para el análisis de una cavidad horizontal calentada por el fondo.

Al igual que para el proceso de convección natural en cavidades con un fluido, para medios porosos se han publicado recopilaciones sobre flujo y transporte de energía, entre los que destacan Nield y Bejan (1992) e Ingham y Pop (1998), en ellos se ya se incluyen trabajos que incluyen el proceso turbulento en medios porosos, mismos que han adquirido importancia en años recientes (Silva y col., 2003; De Lemos y Mesquita, 2003). 


\subsection{Sistema fluido-medio poroso.}

Para dar una visión de la importancia que adquiere el acoplar los problemas de flujo en cavidades formados por un fluido homogéneo, con el fenómeno que ocurren en medios porosos (condiciones de salto en la frontera fluido-medio poroso), a continuación se presenta un resumen de los antecedentes y revisiones recientes, incluyendo los trabajos que consideran los métodos de solución del sistema de ecuaciones.

Se ha realizado un gran número de estudios en sistemas bidimensionales para el proceso de convección natural en sistemas fluido-medio poroso (Whitaker, 1986; Poulikakos y col., 1986; Chen y Chen, 1988; Nishimura y col., 1986; Beckermann y col., 1987,1988; Sathe y col., 1988); dentro de estos estudios se encuentran los que consideran el caso de un fluido superpuesto horizontalmente sobre un medio poroso (Tien y Hong, 1985; Nield, 1977). Por otro lado se encuentran trabajos que consideran un almacenamiento vertical rectangular y dentro de ellos los que toman en cuenta una separación permeable (Poulikakos y Bejan, 1983), o bien aquellos que consideran que las regiones están divididas por un medio impermeable (Tong y Subramanian, 1986).

De los trabajos que consideran el sistema fluido medio poroso, son muy pocos los que analizan un sistema tridimensional (Singh y col., 1993; Jiménez Islas, 1999). Los autores generalmente utilizan aproximaciones para definir las condiciones de frontera en la interregión fluido-medio poroso. La aproximación más frecuente para representar la transferencia de cantidad de movimiento en la región porosa es la que involucra la corrección de Brinkman a la ecuación de Darcy. En este caso las ecuaciones diferenciales que gobiernan la transferencia de cantidad de movimiento en el fluido y el medio poroso son de segundo orden por lo que es posible el acoplamiento matemático. Un ejemplo de esto lo presentaron Singh y col. (1993) mediante un estudio numérico sobre convección natural en una cavidad tridimensional con un sistema fluido-medio poroso utilizando la corrección de Brinkman a la ecuación de Darcy.

En algunos casos cuando es utilizada la corrección de Brinkman, la viscosidad efectiva del medio poroso se considera mediante un parámetro empírico. Recientemente Ochoa-Tapia y Whitaker (1995-a,b) desarrollaron una condición de salto para acoplar la ecuación de Navier-Stokes en la parte fluida con la ecuación de Darcy con la corrección de Brinkman en la región porosa. Estos autores demostraron que no existe ninguna razón teórica para utilizar la viscosidad efectiva como un parámetro empírico. Como una extensión de este trabajo Silva y De Lemos (2003) aplican el procedimiento para analizar el efecto turbulento en la interregión fluido-medio poroso.

Otro método para acoplar las ecuaciones de transferencia de cantidad de movimiento entre un fluido y un medio poroso es hacer uso de expresiones semiempíricas propuestas por 
Beavers y Joseph (1967), estas correcciones aunque sencillas, requieren de la determinación de parámetros empíricos dependientes del medio poroso.

\subsection{Difusión Doble.}

Cuando la convección en una cavidad es resultado de gradientes de temperatura y concentración recibe el nombre de "convección doblemente difusiva” o simplemente "difusión doble”. El fenómeno ocurre en una amplia variedad de campos como la oceanografía, geología y metalurgia. Particularmente el fenómeno de convección de calor y masa en cavidades que contienen un medio poroso ha sido de gran interés debido a sus múltiples aplicaciones como: Reactores catalíticos y almacenamiento de granos.

Nield (1968) realizó uno de los primeros estudios para estudiar el flujo convectivo en arreglos horizontales con condiciones de frontera verticales para concentración y temperatura, éste fue continuado por Khan y Zebib (1981) para una superficie vertical en un medio poroso, Poulikakos (1986) realizó un estudio de difusión doble en medios porosos utilizando la extensión de Brinkman a la ecuación de Darcy; Rudraiah y col. (1982) y Trevisan y Bejan (1985) estudiaron los efectos de los Números de Prandtl, Lewis y Darcy en un sistema consistente de un medio poroso. Lee y col. (1988) reportan los efectos de la difusión de calor y masa en sistemas homogéneos (aire y agua principalmente) formados por superficies verticales. Trevisan y Bejan (1987), realizaron un estudio teórico y numérico de transferencia de masa en un sistema bidimensional consistente de un medio poroso calentado en la parte inferior. El mismo sistema fue estudiado por Rosenberg y Spera (1992) considerando diferentes condiciones de frontera e iniciales. Un estudio experimental de difusión doble en medios porosos lo llevaron a cabo Murray y Chen (1989) encontrando buena concordancia de desarrollos teóricos con los experimentales. Mamou y col. (1998) estudiaron la estabilidad numérica en el proceso de convección doble para una cavidad porosa bidimensional sujeto al movimiento horizontal, provocado por gradientes opuestos de temperatura y concentración. Los estudios de estos autores permitieron determinar el valor crítico del Número de Rayleigh y la estructura del flujo dentro de la cavidad. Gobin y col. (1998), se enfocaron al estudio del fenómeno de difusión doble en una cavidad bidimensional consistente de un fluido y un medio poroso, los autores utilizan la formulación matemática de un dominio.

Sezai y Mohamad (1999) desarrollaron un modelo matemático basado en la extensión de Brinkman a la ecuación de Darcy, para el estudio de la difusión doble en cavidades tridimensionales. El sistema considerado es una cavidad con un medio poroso impregnado de un fluido y sujeto a gradientes horizontales de temperatura y concentración impuestas a lo largo de dos paredes verticales opuestas. Por otro lado Mahidjiba y col. (2000) enfocan su estudio de difusión doble a considerar condiciones de frontera independientes para masa y 
energía, mostrando resultados numéricos para diversas condiciones de frontera mediante perfiles de temperatura y concentración.

Sezai y Mohamad (2000) estudiaron una cavidad tridimensional con una sóla región (fluida), y con paredes isotérmicas con gradientes de temperatura y concentración en paredes opuestas, haciendo variar diferentes parámetros como el Número de Rayleigh, Lewis, y la relación de flotación. Gracias a los resultados de este trabajo se pudo demostrar que el flujo convectivo con doble difusión con fuerzas de flotación opuestas es estrictamente tridimensional para ciertos rangos de los parámetros. Los resultados para cavidades conteniendo fluido fueron contrastados con aquellos obtenidos para una cavidad tridimensional consistente de un medio poroso (Sezai y Mohamad, 1999; Diersch y Kolditz, 1998).

Posteriormente, un trabajo sobre difusión doble fue realizado por Mamou y col. (2001), los autores enfocaron su análisis al estudio de estabilidad numérica en cavidades bidimensionales conteniendo un fluido. Bajo el mismo enfoque pero aplicado a un medio poroso fue realizado un estudio años antes por Mamou y col. (1998), Otro trabajo relacionado con doble difusión es el estudio analítico realizado en una cavidad con un lecho horizontal con gradientes de temperatura y concentración verticales (Kalla y col., 2001 a), en este caso, el estudio se enfoca a los efectos cruzados de transferencia de masa y calor al combinar las condiciones de frontera en paredes opuestas de una cavidad bidimensional. Gobin y col. (2005) presentan un estudio matemático basado en la formulación de un dominio, los autores presentan resultados ara una cavidad bidimensional con un arreglo poroso orientado verticalmente, el estudio numérico considera el análisis de las fuerzas de flotación, la transferencia de masa y calor como función de la permeabilidad del medio poroso (efectos sobre los números de Nuselt y Lewis en función del número de Darcy).

En cuestiones de métodos de solución, Wang y col. (2003) obtienen una solución explícita (previamente planteada por Liao, 1995; 1997; 1999 a,b; 2002 y Liao y Campo 2002) al fenómeno de difusión doble en una pared vertical porosa. Chakraborty y Dutta (2003) presentan un modelo matemático para el proceso de doble difusión en la solidificación de una mezcla binaria en una cavidad tridimensional enfriada lateralmente, los resultados muestran variaciones importantes al comparar los resultados obtenidos en dos dimensiones.

Para analizar los aspectos geométricos, Costa (2004) utiliza las formulaciones presentadas por Chamkha (2002); Kalla y col. (2001 a,b) y Bera y Khalili (2002) para presentar un modelo de difusión doble para una geometría paralelográmica haciendo variar la relación de aspecto y el ángulo de inclinación para determinar las condiciones para las cuales se tiene una mayor transferencia de energía. Jiménez y col. (2004) presentan un estudio numérico para el fenómeno de difusión doble en una cavidad cilíndrica considerando un sistema bidimensional, los autores muestran la aplicación directa del fenómeno al 
almacenamiento de granos alimenticios, específicamente para analizar la dinámica del almacenamiento de sorgo, los autores establecen el tiempo de almacenamiento para obtener el equilibrio entre el contenido de humedad en el grano y la humedad del aire, concluyendo la necesidad de análisis considerando las tres dimensiones.

\subsection{Condiciones en la interregión.}

Las características del tipo de flujo de fluidos y la transferencia de calor en la interfase de un sistema que consta de dos regiones: un fluido y un medio poroso ha tenido gran interés por el número de aplicaciones en ingeniería tales como el enfriamiento electrónico, enfriamiento por transpiración, proceso de secado, aislamiento térmico, colectores solares, extracción de crudo e ingeniería geotérmica.

Uno de los primeros trabajos que se enfocaron a estudiar las condiciones de frontera para cantidad de movimiento fue presentado por Beavers y Joseph (1967). Los autores desarrollaron experimentos y detectaron un deslizamiento en la velocidad en la cercanía de la interregión. Por su parte, Neale y Nader (1974) proponen una continuidad en la velocidad y los gradientes en la interfase al introducir un término de segundo orden (término de Brinkman) en la ecuación de cantidad de movimiento para modelar el medio poroso. Posteriormente Vafai y Kim (1990) presentaron una solución exacta para el flujo en la interfase entre un fluido y un medio poroso incluyendo los efectos inerciales. En estudios posteriores en los que se analizan los efectos de la interregión entre un medio poroso y un fluido homogéneo, fue desarrollada la condición de salto para el flux, basada en la forma no-local de las ecuaciones de promedio para el transporte de cantidad de movimiento (Ochoa-Tapia y Whitaker, 1995a,b y 1998a) y para transferencia de calor (Ochoa-Tapia y Whitaker, 1997, 1998b). En la condición de salto para la transferencia de cantidad de movimiento Ochoa-Tapia y Whitaker (1995a) muestran resultados teóricos, los cuales fueron comparados con datos experimentales (Ochoa-Tapia y Whitaker, 1995b), esta propuesta de la condición de salto para la transferencia de cantidad de movimiento, ha sido utilizada en diferentes trabajos para su comprobación y/o aplicación a diferentes sistemas por Kuznetsov (1996,1997,1998a,b y 1999). De igual forma, se han presentado otras condiciones de frontera donde los efectos inerciales llegan a ser importantes en la región frontera (Ochoa-Tapia y Whitaker, 1998a).

Los avances en el desarrollo de condiciones de salto no se limitan a la ecuación de transferencia de cantidad de movimiento, así pues, la condición de salto que se aplica en la frontera entre un medio poroso y un fluido cuando la condición de equilibrio térmico local no es válida, fue igualmente desarrollada por Ochoa-Tapia y Whitaker (1997), estos autores aplican las ecuaciones de transporte separadamente para describir el comportamiento de cada una de las fases y encontraron una condición que contiene un término superficial de exceso de intercambio de calor que controla la forma de cómo es distribuido el flux total entre ambas 
fases. Posteriormente Ochoa-Tapia y Whitaker (1998b) desarrollaron la condición de salto cuando la condición de equilibrio térmico es impuesta y encontraron que la forma no local simplifica al modelo clásico de una ecuación para el transporte de energía. Alazmi y Vafai (2001), han analizado diferentes tipos de condiciones interfaciales, catalogándolas como condiciones interfaciales con y sin deslizamiento, dichos autores han marcado las diferencias entre las diferentes formulaciones poniendo especial interés en las presentadas por OchoaTapia y Whitaker (1998b).

Por otro lado, el proceso de transporte de masa en la frontera de un medio poroso y un fluido homogéneo ocurre en una amplia variedad de aplicaciones tecnológicas y por lo tanto ha sido objeto de gran variedad de estudios. Como ejemplos se tienen las columnas de adsorción (Aris, 1959), y el fenómeno de dispersión convectiva que resulta como consecuencia de la transferencia de masa interfacial en cavidades cilíndricas (Lenhoff y Lightfoot, 1984) incluyendo aquellas que consideran reacciones de primer orden (Lenhoff y Lightfoot, 1986). La cromatografía de flujo radial para la separación de varios productos biológicos ha sido reportado por diferentes autores (Chen and Hou, 1985; Saxena y col., 1987; Huang y col., 1988; Lee y col., 1990; Saxena y Weil, 1987). Una extensión de las separaciones cromatográficas puede ser encontrada en los reactores empacados de lecho fijo con flujo radial. Estos son usados en una gran variedad de aplicaciones industriales como la síntesis de metanol, regeneración de catalizadores, desulfuración en fase vapor (Balakotaiah y Luss, 1981), por mencionar algunas. Los trabajos para determinar la condición de salto para la transferencia de masa son limitados y específicos para cada problema (Ochoa y Soria, 1995), lo anterior muestra la importancia del desarrollo de una condición de salto para la ecuación de transferencia de masa en la interregión fluido-medio poroso.

En 2003, Goyeau y col. presentaron un estudio sobre la condición interfacial entre un fluido y un medio poroso basado en la formulación de Beavers y Joseph y la formulación de un dominio, los autores desarrollaron un modelo que incluye un coeficiente de deslizamiento dependiente de la velocidad del fluido y de las propiedades efectivas del medio poroso, en sus resultados muestran que las formulaciones de uno y dos dominios son equivalentes cuando el medio poroso es homogéneo. Dentro de sus conclusiones los autores consideran su análisis como un paso intermedio para obtener el problema de cerradura para el problema del acoplamiento interfacial de las ecuaciones de transferencia de cantidad de movimiento.

\section{RESUMEN:}

La revisión bibliográfica desarrollada muestra que el flujo convectivo en cavidades y en medios porosos ha sido ampliamente estudiado y los avances que se tienen van desde aspectos teóricos hasta geométricos y numéricos. Para el fenómeno de difusión doble se dispone de estudios realizados en cavidades conteniendo un fluido o un medio poroso, sin 
embargo cuando se tiene que acoplar un sistema fluido-medio poroso los avances son más limitados, pues los estudios se reducen a analizar sistemas bidimensionales utilizando la extensión de Brinkman a la ecuación de Darcy.

Por otro lado, existen algunos autores que han desarrollado trabajos teóricos para el acoplamiento de las ecuaciones, principalmente para las ecuaciones de transferencia de cantidad de movimiento y las de energía. Por lo que el presente trabajo tiene tres objetivos principales: Primero se pretende analizar los efectos tridimensionales de la convección natural y hacer un estudio comparativo entre las formas de acoplar la ecuación de transferencia de cantidad de movimiento (Darcy y extensión de Brinkman). El segundo objetivo es realizar una comparación del modelado con un sistema bidimensional, y en el tercero el desarrollo de la condición de salto para la transferencia de masa en un sistema fluido medio poroso. 


\section{Capítulo 2}

\section{Planteamiento del modelo.}

Considerando la revisión bibliográfica sobre flujo en cavidades, flujo en medios porosos y condiciones de salto presentadas en el capítulo anterior y teniendo en cuenta los objetivos específicos, en el presente capítulo se desarrollan las ecuaciones utilizadas en los modelos matemáticos que describen la convección natural en cavidades causada por gradientes de temperatura y masa.

Se ha dividido este planteamiento considerando cada una de las ecuaciones utilizadas en ambas regiones, es decir, se presenta el desarrollo de cada una de las ecuaciones de transporte para cada una de las regiones y se establecen las condiciones de frontera e interregionales así como el cambio a variables adimensionales. Posteriormente para su solución se replantea la ecuación de cantidad de movimiento a la formulación vorticidadvector potencial. Finalmente se presenta un resumen de los conjuntos de ecuaciones utilizadas para cada uno de los modelos.

Dentro de los objetivos del presente trabajo se encuentra el desarrollo de la condición de salto para la transferencia de masa en el fenómeno de difusión pasiva. El procedimiento detallado de este desarrollo se presenta en el Apéndice A. Para el caso de la condición de salto de las ecuaciones de transferencia de cantidad de movimiento y energía se presentan los resultados finales ya que fueron desarrollados previamente (Ochoa Tapia y Whitaker, 1995a,b,c, Ochoa y Whitaker, 1997,1998a,b). De cualquier forma, los fundamentos del método del promedio volumétrico se presentan en los apéndices A y B. 


\subsection{Sistema.}

Aunque el modelo matemático se desarrollará de forma general, se tomará un sistema simplificado para realizar las pruebas al modelo numérico. Este sistema está formado por una cavidad rectangular, la cual contiene un fluido (fase $\beta$ ) y un material granular (fase $\sigma$ ) distribuido en dos regiones como se muestra en la Figura 2.1. La región superior (región $\eta$ ) está constituida por el fluido homogéneo y la parte inferior por un medio poroso (región $\omega$ ), este último está formado por las fases $\sigma$ y $\beta$. El sistema con las dimensiones y ejes utilizados se seleccionó de tal forma que el eje $x$ está dirigido en la dirección del vector gravedad, mientras que los ejes $y$ y $z$ se encuentran en el plano horizontal como se muestra en la Figura 2.1.

El medio poroso se considera macroscópicamente homogéneo, mientras que la interregión se establece horizontal y permeable de tal manera que existe flujo entre ambas regiones. Adicionalmente se considera flujo incompresible con propiedades físicas constantes excepto la densidad, la simplificación anterior es conocida como la aproximación de Boussinesq (Dong y Li , 2004; Bird y col., 2002; Ravi y col., 1994; Singh y col., 1993; Gray y Giorgini, 1976), misma que se explica posteriormente.

Para evitar la complejidad que se tiene al considerar los detalles de la geometría del medio poroso, se plantean las ecuaciones en términos de ecuaciones de transporte promedio para ambas regiones. De acuerdo con Ochoa-Tapia y Whitaker (1995a,b,c) las ecuaciones promedio en la región fluida tienen la misma forma que las ecuaciones puntuales de transporte, debido a que las restricciones de escalas establecidas por el método de promedio volumétrico son satisfechas (Whitaker, 1999), con respecto al promedio de las ecuaciones en la región porosa, a continuación se describe el procedimiento utilizado.

\subsection{Ecuaciones del medio poroso.}

La forma usual para la obtención de las ecuaciones en medios porosos en términos de variables macroscópicas comienza planteando las ecuaciones estándar que gobiernan la región fluida, posteriormente se obtienen las ecuaciones macroscópicas mediante un promedio sobre el volumen o el área. Existen varias formas para realizar el promediado, entre éstas se encuentran el promedio espacial y el promedio estadístico. En la aproximación espacial, una variable macroscópica se define como un promedio apropiado sobre un elemento de volumen suficientemente grande, la longitud del elemento diferencial es mucho más grande que la del poro, pero considerablemente inferior que la longitud del dominio del fluido. En la aproximación estadística el promedio se lleva a cabo sobre un conjunto de la estructura del poro, el cual tiene su equivalente macroscópico. Una dificultad de esta aproximación es que usualmente la información estadística acerca del medio poroso se basa sobre una muestra, y esto es posible solamente si se asume homogeneidad estadística. 


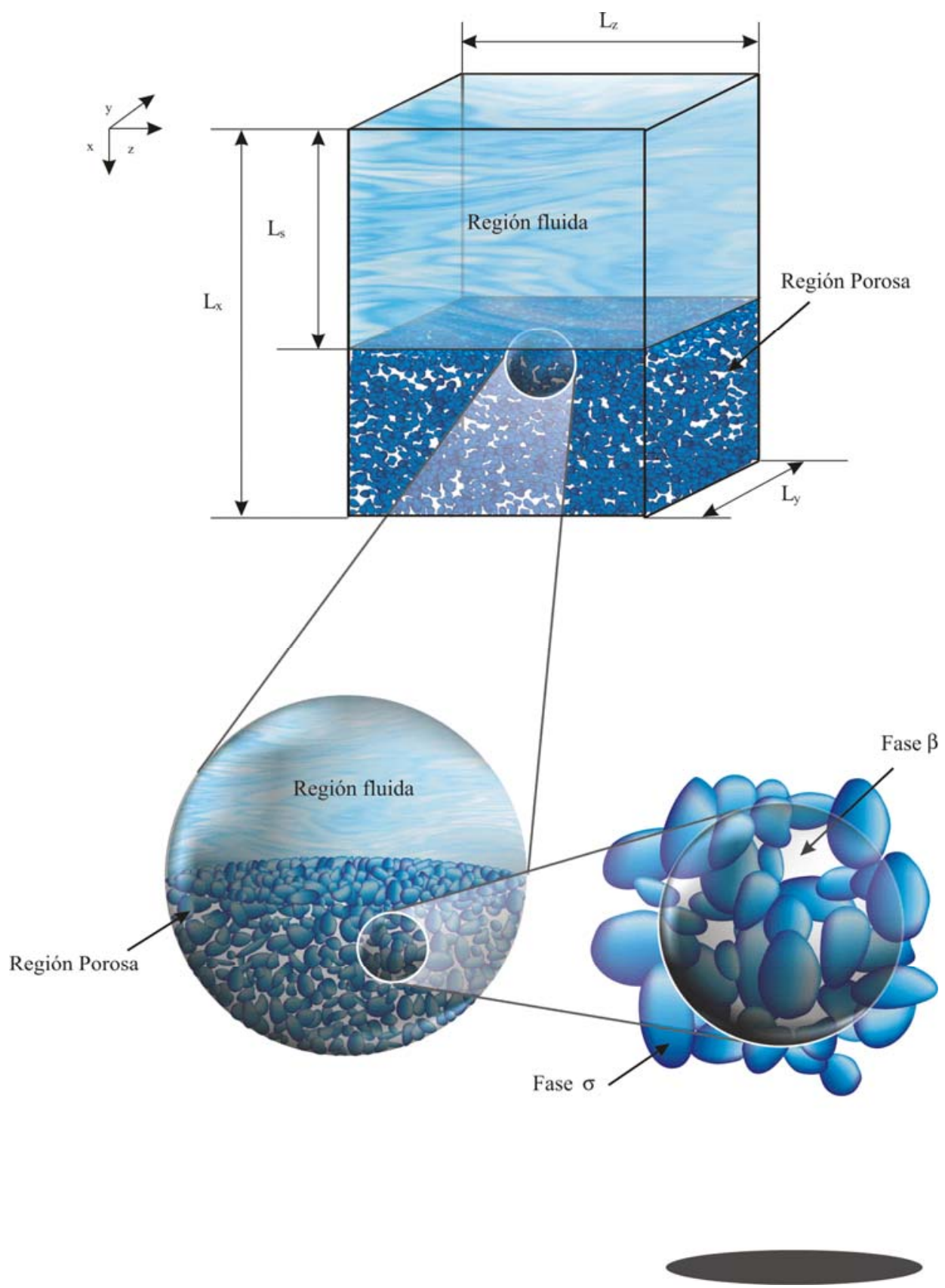

Figura 2.1.- Cavidad y regiones utilizadas para el desarrollo de las ecuaciones. 
En el desarrollo del presente trabajo se utilizó un promedio espacial, considerando el uso del método del promedio volumétrico (Howes y Whitaker, 1985; Whitaker, 1999) para la obtención de las ecuaciones en su forma promediada, este método ya ha sido previamente probado para describir fenómenos de transferencia de calor (Carbonell y Whitaker, 1984), masa (Whitaker,1967; Ochoa-Tapia y col.,1994) y cantidad de movimiento en medios porosos (Whitaker, 1966, 1969, 1986 a, b).

\subsection{Transferencia de calor.}

En la convección natural, una de las causas que propicia el movimiento del fluido dentro de la cavidad lo constituye la diferencia de temperatura que existe entre las paredes, y es precisamente el problema en particular que aborda el presente trabajo. En las secciones siguientes se plantean las ecuaciones de energía y las simplificaciones realizadas para cada una de las regiones consideradas.

\subsubsection{Ecuación de transferencia de calor en la región fluida.}

En la región fluida $(\eta)$, para la ecuación de transferencia de calor en régimen permanente se consideran los términos convectivo y conductivo. Como se mencionó anteriormente, todas las propiedades se consideran constantes, excepto la densidad en el término de flotación. La forma dimensional para la ecuación de transferencia de energía utilizada es la siguiente:

$$
\underbrace{\left\langle\mathbf{v}_{\beta}^{\prime}\right\rangle_{\eta} \cdot \nabla^{\prime}\left\langle T_{\beta}\right\rangle_{\eta}}_{\text {Término convectivo }}=\underbrace{\left(\alpha_{\beta}\right)_{\eta} \nabla^{\prime 2}\left\langle T_{\beta}\right\rangle_{\eta}}_{\text {Término conductivo }}
$$

Donde $\left(\alpha_{\beta}\right)_{\eta}$ es la difusividad térmica del fluido, $\left\langle\mathbf{v}_{\beta}^{\prime}\right\rangle_{\eta} \mathrm{y}\left\langle T_{\beta}\right\rangle_{\eta}$ representan el promedio superficial de la velocidad y temperatura en la región fluida respectivamente. La definición de los diferentes promedios utilizados se presentan en el desarrollo de la condición de salto para la transferencia de masa (Apéndice A).

La ecuación (2.1) se expresa en función de variables primitivas dimensionales $\left(\left\langle\mathbf{v}_{\beta}^{\prime}\right\rangle \mathrm{y}\left\langle T_{\beta}\right\rangle_{\eta}\right)$. La forma adimensional se obtiene mediante la utilización de los siguientes grupo de variables: 


$$
\begin{array}{cc}
\mathbf{v}_{\lambda}=\frac{\left\langle\mathbf{v}_{\beta}^{\prime}\right\rangle_{\lambda} L_{x}}{\left(\alpha_{\beta}\right)_{\eta}} & \text { para } \lambda=\eta, \omega \\
\theta_{\lambda}=\frac{\left\langle T_{\beta}\right\rangle_{\lambda}-T_{\text {ref }}}{\Delta T} & \text { para } \lambda=\eta, \omega \\
\nabla=L_{x} \nabla^{\prime} & \\
\nabla^{2}=L_{x}^{2} \nabla^{\prime 2} & \\
X=\frac{x}{L_{x}} & \\
Y=\frac{y}{L_{x}} &
\end{array}
$$

En las ecuaciones (2.2) y $(2.4 \mathrm{a}, \mathrm{b})$ se ha tomado como longitud característica $L_{x}$, que es la altura de la cavidad (Figura 2.l). Aplicando las variables anteriores a la ecuación (2.1) se tiene la versión adimensional para la transferencia de energía.

$$
\underbrace{\nabla \cdot\left(\mathbf{v}_{\eta} \theta_{\eta}\right)}_{\text {Convección }}=\underbrace{\nabla^{2} \theta_{\eta}}_{\text {Conducción }}
$$

En la ecuación (2.5) desaparece el símbolo de promedio “ \langle\rangle ” debido a la definición de las variables adimensionales, la decisión se tomó para simplificar la notación en las ecuaciones, sin embargo, hay que tener en cuenta que la ecuación resultante considera variables promedio.

\subsubsection{Ecuación de transferencia de calor en la región porosa.}

Para la ecuación de energía en la región porosa se utilizó el resultado del desarrollo del modelo de una ecuación para la transferencia de calor en medios porosos, el desarrollo se encuentra en los trabajos de Whitaker (1991, 1992b)

$$
\underbrace{\left\langle\mathbf{v}_{\beta}^{\prime}\right\rangle_{\omega} \cdot \nabla^{\prime}\left\langle T_{\beta}\right\rangle_{\omega}}_{\text {Término convectivo }}=\underbrace{\left(\alpha_{\beta}\right)_{\omega} \nabla^{\prime 2}\left\langle T_{\beta}\right\rangle_{\omega}}_{\text {Término conductivo }}
$$

Utilizando las variables adimensionales especificadas por las ecuaciones. (2.2)-(2.4), la ecuación de energía en la región porosa queda como:

$$
\underbrace{\nabla \cdot\left(\mathbf{v}_{\omega} \theta_{\omega}\right)}_{\text {Convección }}=\underbrace{K_{\omega} \nabla^{2} \theta_{\omega}}_{\text {Conducción }}
$$


Donde $K_{\omega}$ representa la relación de difusividades térmicas entre la región porosa y fluida, la cual está expresada por:

$$
K_{\omega}=\frac{\left(\alpha_{\beta}\right)_{\omega}}{\left(\alpha_{\beta}\right)_{\eta}}
$$

\subsubsection{Condiciones de frontera y ecuación en la interregión para la transferencia de calor.}

Las condiciones de frontera en las paredes se imponen dependiendo de las pruebas que se realicen, éstas se especifican en el Capítulo 4. De manera general se podrán manejar condiciones de pared o áreas isotérmicas sobre paredes, consideraciones de gradientes de temperatura en paredes opuestas en combinación con paredes adiabáticas (Dirichlet, Newman y Cauchy o Robins), específicamente:

$$
\begin{aligned}
& \left(\theta_{\lambda}\right)_{b}=a \quad \lambda=\eta, \omega \quad \text { Dirichlet } \\
& \left(\frac{d \theta_{\lambda}}{d n}\right)_{b}=a \quad \lambda=\eta, \omega \quad \text { Newman } \\
& \left(\frac{d \theta_{\lambda}}{d n}\right)_{b}+a\left(\theta_{\lambda}\right)_{b}=c \quad \lambda=\eta, \omega \quad \text { Cauchy o Robins }
\end{aligned}
$$

Donde el subíndice $b$ indica cualquier pared de la cavidad, $n$ la componente normal a la pared $b$, mientras que $a$ y $c$ son constantes.

Una vez que la conductividad térmica en el medio poroso ha sido determinada, la aplicación de las condiciones de frontera en la interregión es usualmente sencilla. En la zona que divide el medio poroso y el fluido se puede establecer la continuidad de la temperatura, aplicando la suposición de equilibrio térmico local (Haddad y col., 2004; Duval y col., 2004; Whitaker, 1986b, 1991 y Quintard y Whitaker, 1995) y la continuidad de las componentes normales al flux.

$$
\begin{aligned}
& \theta_{\omega}=\theta_{\eta} \quad \text { Continuidad en el campo en } x=L_{S} \\
& -\mathbf{n}_{\omega \eta} \cdot K_{\omega} \nabla \theta_{\omega}=-\mathbf{n}_{\omega \eta} \cdot \nabla \theta_{\eta} \quad \text { Continuidad en el flux en } x=L_{S}
\end{aligned}
$$




\subsection{Transferencia de masa en el problema de difusión doble.}

El problema de convección doblemente difusiva se considera como un proceso simultáneo de transferencia de calor y masa debido a las fuerzas de flotación. Los gradientes que provocan las fuerzas son inducidos por efectos combinados de temperatura y concentración de especies presentes en el fluido saturado.

Debido a la inclusión simultánea de los términos de difusión térmica y másica surge un efecto importante en la convección debido a que el calor se difunde más rápidamente que una substancia disuelta, esto sin considerar la posibilidad de la difusión cruzada: efecto Soret y Dufour (Postelnicu, 2004). El efecto Soret se refiere al flux másico producido por gradientes de temperatura y el efecto Dufour a efectos de flux de calor producidos por gradientes de concentración, los cuales se consideran despreciables en el presente trabajo.

\subsubsection{Ecuación de transferencia de masa en la región fluida.}

Para la ecuación de transferencia de masa en la región fluida se consideran los términos convectivo y difusivo, la ecuación que incluye estos dos términos en función de variables primitivas queda expresada mediante:

$$
\underbrace{\left\langle\mathbf{v}_{\beta}^{\prime}\right\rangle_{\eta} \cdot \nabla^{\prime}\left\langle C_{A \beta}\right\rangle_{\eta}}_{\text {Término convectivo }}=\underbrace{\left(\mathrm{D}_{\beta}\right)_{\eta} \nabla^{\prime 2}\left\langle C_{A \beta}\right\rangle_{\eta}}_{\text {Término difusivo }}
$$

Utilizando las variables adimensionales definidas por las ecuaciones (2.2)-(2.4) junto con la definición adimensional para la concentración definida por la ecuación (2.13), se tiene la ecuación adimensional para la transferencia de masa en la región fluida:

$$
\mathbf{v}_{\eta} \cdot \nabla \phi_{\eta}=\frac{1}{L e_{\eta}} \nabla^{2} \phi_{\eta}
$$

Donde

$$
\phi_{\lambda}=\frac{\left\langle C_{A \beta}\right\rangle_{\lambda}-C_{A \beta_{\text {ref }}}}{\Delta C} \quad \text { para } \lambda=\eta, \omega
$$

En la ecuación (2.12) se ha incorporado el número de Lewis $\left(L e_{\eta}\right)$, el cual considera la relación entre las difusividades térmica y másica mediante: 


$$
L e_{\eta}=\frac{\left(\alpha_{\beta}\right)_{\eta}}{\left(\mathrm{D}_{\beta}\right)_{\eta}}
$$

\subsubsection{Ecuación de transferencia de masa en la región porosa.}

De igual forma que en la región fluida, la forma dimensional para la ecuación de transferencia de masa en el medio poroso se expresa en términos de variables promedio. Se considera la forma más simple de transporte convectivo en un medio poroso que es el que toma en cuenta la convección pasiva y difusión en un medio poroso rígido e impermeable. El término pasivo se refiere a que no se considera adsorción o reacción en la interfase sólidolíquido. Este problema ya ha sido investigado previamente por Whitaker (1999). La ecuación resultante en términos de variables primitivas promedio es la siguiente:

$$
\left\langle\mathbf{v}_{\beta}^{\prime}\right\rangle_{\omega} \cdot \nabla^{\prime}\left\langle C_{A \beta}\right\rangle_{\omega}=\mathbf{D}_{e f f} \nabla^{\prime 2}\left\langle C_{A \beta}\right\rangle_{\omega}
$$

Donde $\mathbf{D}_{\text {eff }}$ es la difusividad efectiva, y su dependencia con respecto a la difusividad en la fase $\beta$ se muestra en el Apéndice B.

Haciendo el cambio a variables adimensionales utilizando las ecuaciones (2.2)-(2.4), (2.13) y (2.14), la ecuación (2.15) se reescribe como:

$$
\mathbf{v}_{\omega} \cdot \nabla \phi_{\omega}=\frac{1}{L e_{\omega}} \nabla^{2} \phi_{\omega}
$$

El número de Lewis $\left(L e_{\omega}\right)$ en la región porosa involucra la relación de la conductividad térmica en la región fluida con respecto a la difusividad másica en la región porosa:

$$
L e_{\omega}=\frac{\left(\alpha_{\beta}\right)_{\eta}}{D_{\text {eff }}}
$$

\subsubsection{Condiciones de frontera y ecuación en la interregión para la transferencia de masa.}

Al igual que en la ecuación de energía, para la ecuación de transferencia de masa las condiciones de frontera en las paredes se impondrán dependiendo de las pruebas que se realicen, éstas se especifican en el Capítulo 4. En forma general los tipos de condiciones de 
frontera que considera el modelo para las paredes, están dadas por formas similares a las ecuaciones (2.9).

Uno de los desarrollos importantes en el presente trabajo es la deducción de una condición de salto para el proceso de difusión pasiva en la zona que divide el medio poroso y el fluido, los detalles del desarrollo para esta condición se presentan en el Apéndice A, los resultados finales son:

$$
\begin{gathered}
\phi_{\omega}=\phi_{\eta} \\
-\mathbf{n}_{\omega \eta} \cdot\left[\mathbf{D}_{\omega}^{*} \cdot \nabla \phi_{\omega}-\mathbf{D}_{\eta} \nabla \phi_{\eta}\right]=0
\end{gathered}
$$

\subsection{Transferencia de cantidad de movimiento.}

En el caso de la transferencia de cantidad de movimiento se utilizará la ecuación de Navier-Stokes para la región fluida, mientras que para la región porosa la tendencia general en el modelamiento de la convección libre está basada en la formulación derivada de la ecuación de Darcy o en alguna de sus extensiones (Nield y Bejan, 1992).

En sistemas donde la convección natural es el transporte predominante, la variación de la densidad con la temperatura tiene una gran importancia y es conveniente modificar la ecuación de movimiento para tener en cuenta los efectos de flotación, esto es, la inclusión del término gravitacional $(\rho \mathbf{g})$. Debido a lo anterior se necesita una ecuación de estado para las ecuaciones de masa, cantidad de movimiento y energía, sin embargo esto ocasionaría que las ecuaciones diferenciales aumenten su grado de no linealidad y por consecuencia la solución se complica.

La forma práctica que han utilizado muchos autores para resolver este problema consiste en suponer las propiedades constantes excepto la densidad en el término de fuerzas volumétricas (Nield y Bejan, 1992; Nield, 1977; Gebhart y col., 1988 y Bird y col., 2002), a esta simplificación se le conoce como la aproximación de Boussinesq, estudios sobre la validez de esta aproximación han sido realizados por Gray y Giorgini (1976), Jiménez-Islas y col. (2004).

Sin embargo, en la convección con doble difusión el acoplamiento se lleva a cabo debido a que la densidad de la mezcla líquida depende no solo de la temperatura, sino también de la concentración (y en general, de la presión). En este caso la aproximación de Boussinesq se continuó aplicando teniendo en cuenta que para cambios isobáricos suficientemente pequeños en temperatura y concentración la densidad del fluido tiene una dependencia lineal 
con respecto a la temperatura y concentración, quedando la siguiente aproximación en términos de variables dimensionales promedio como:

$$
\rho \cong \rho_{\beta}\left[\beta_{\theta}\left(\langle T\rangle-\left\langle T_{r e f}\right\rangle\right)-\beta_{\phi}\left(\left\langle C_{A \beta}\right\rangle-\left\langle C_{\text {Aref }}\right\rangle\right)\right]
$$

Donde $\beta_{\theta}$ y $\beta_{\phi}$ son los coeficientes de expansión térmica y másica respectivamente, y ambos son evaluados con respecto al estado de referencia:

$$
\begin{array}{ll}
\beta_{\phi}=-\frac{1}{\rho_{0}}\left(\frac{\partial \rho_{\beta}}{\partial\left\langle C_{A \beta}\right\rangle}\right)_{P, T} \quad \text { Coeficiente de expansión másica } \\
\beta_{\theta}=-\frac{1}{\rho_{0}}\left(\frac{\partial \rho_{\beta}}{\partial\langle T\rangle}\right)_{P, C} \quad \text { Coeficiente de expansión térmica }
\end{array}
$$

\subsubsection{Ecuación para la transferencia de cantidad de movimiento en la región fluida.}

Con las observaciones realizadas al término de flotación, la ecuación de cantidad de movimiento para la región fluida (ecuación de Navier-Stokes) en términos de variables primitivas en su forma dimensional es:

$$
\begin{aligned}
\underbrace{\nabla^{\prime} \cdot\left(\left\langle\mathbf{v}_{\beta}^{\prime}\right\rangle_{\eta}\left\langle\mathbf{v}_{\beta}^{\prime}\right\rangle_{\eta}\right)}_{\text {Convección }} & =-\underbrace{\frac{1}{\rho_{\beta}} \nabla^{\prime}\left\langle p_{\beta}^{\prime}\right\rangle_{\eta}^{\beta}}_{\text {Fuerzas de presión }}+\underbrace{v_{\beta} \nabla^{\prime 2}\left\langle\mathbf{v}_{\beta}^{\prime}\right\rangle_{\eta}}_{\text {Transporte viscoso }} \\
& +\underbrace{\mathbf{i} g \rho_{\beta}\left[\beta_{\theta}\left(\left\langle T_{\beta}\right\rangle_{\eta}-T_{r e f}\right)-\beta_{\phi}\left(\left\langle C_{A \beta}\right\rangle_{\eta}-C_{A \beta_{\text {ref }}}\right)\right]}_{\text {Flotación }}
\end{aligned}
$$

Donde $\left\langle p_{\beta}^{\prime}\right\rangle_{\eta}^{\beta}$ representa el promedio intrínseco para la presión (ver Apéndice A).

Expresando la ecuación (2.20) en términos de variables adimensionales definidas por las ecuaciones (2.2), (2.3) y (2.13) para las variables vector velocidad, temperatura y concentración respectivamente e incluyendo la adimensionalización de la presión, definida mediante:

$$
p_{\lambda}=\frac{\left\langle p_{\beta}^{\prime}\right\rangle_{\lambda} L_{x}^{2}}{\left(\alpha_{\beta}\right)_{\lambda} \mu_{\beta}} \quad \text { para } \lambda=\eta, \omega
$$


Con lo anterior, la ecuación de Navier-Stokes en términos de la presión adimensional queda expresada por:

$$
\nabla \cdot\left(\mathbf{v}_{\eta} \mathbf{v}_{\eta}\right)=-\frac{\mu_{\beta}}{\rho_{\beta}\left(\alpha_{\beta}\right)_{\eta}} \nabla p_{\eta}+\frac{v_{\beta}}{\left(\alpha_{\beta}\right)_{\eta}} \nabla^{2} \mathbf{v}_{\eta}+\mathbf{i}\left[\frac{g \beta_{\theta} \Delta T L_{x}^{3}}{\left(\alpha_{\beta}\right)_{\eta}^{2}} \theta-\frac{g \beta_{\phi} \Delta C_{A} L_{x}^{3}}{\left(\mathrm{D}_{\beta}\right)_{\eta}\left(\alpha_{\beta}\right)_{\eta}} \phi\right]
$$

Reordenando la ecuación (2.22) junto con la incorporación de los siguientes números adimensionales

Número de Rayleigh térmico

$$
R a_{\theta}=\frac{g \beta_{\theta} \Delta T L_{x}^{3}}{\left(\alpha_{\beta}\right)_{\eta} v_{\beta}}
$$

Número de Rayleigh másico

$$
R a_{\phi}=\frac{g \beta_{\phi} \Delta C_{A} L_{x}^{3}}{\left(\mathrm{D}_{\beta}\right)_{\eta} v_{\beta}}
$$

Número de Prandtl

$$
\operatorname{Pr}=\frac{v_{\beta}}{\left(\alpha_{\beta}\right)_{\eta}}=\frac{\mu_{\beta}}{\rho_{\beta}\left(\alpha_{\beta}\right)_{\eta}}
$$

Introduciendo el número de Lewis definido por la ecuación (2.14), la ecuación (2.22) en términos de 4 números adimensionales es:

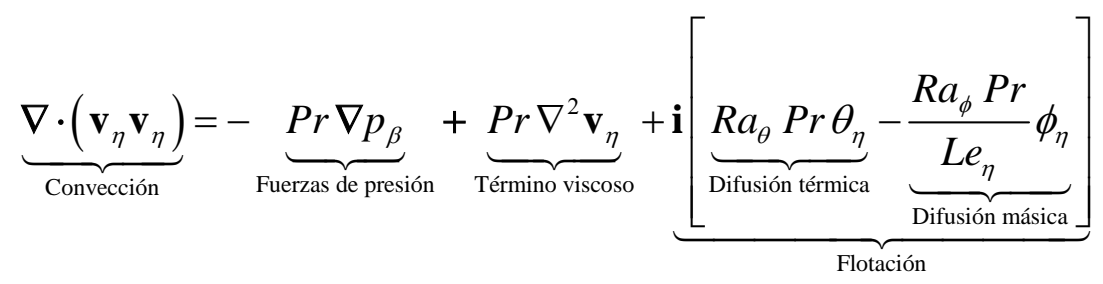

En la ecuación (2.25) $R a_{\theta}$ y $R a_{\phi}$ incluyen la difusividad térmica y difusividad másica respectivamente, estos números afectan al término de flotación. Puede verse que el proceso convectivo está sujeto a dos términos difusivos, razón por la cual este fenómeno recibe el nombre de convección doblemente difusiva. 


\section{ECUACIÓN DE CONTINUIDAD}

Adicionalmente a la ecuación de cantidad de movimiento, se tiene que satisfacer la ecuación de continuidad, para el caso de un flujo incompresible la ecuación en términos de variables adimensionales es:

$$
\nabla \cdot \mathbf{v}_{\eta}=0
$$

El desarrollo de la forma promediada de la ecuación de continuidad se puede consultar en los trabajos reportados por Ochoa-Tapia y Whitaker (1995 a, b).

\subsubsection{Ecuación para la transferencia de cantidad de movimiento en la región porosa.}

Para establecer el modelo de la transferencia de cantidad de movimiento en la región porosa, se cuenta con varios estudios (Chan y col., 1970; Hirt y Cook, 1972; Ettefagh y col., 1991; Holst y Aziz, 1972; Hickox y col., 1996; Jiménez-Islas y col., 1999; Lauriat y Prasat, 1987). En la presente sección se discutirán algunas de las formas de la ecuación de cantidad de movimiento consideradas como una analogía a la ecuación de Navier-Stokes en el medio poroso. En particular se pondrá especial interés en la ley de Darcy y en una de sus extensiones denominada como la corrección de Brinkman a la ecuación de Darcy o simplemente como la corrección de Brinkman.

\section{a) Ecuación de Darcy.}

La ecuación fundamental para representar el movimiento de un fluido a través de un medio poroso es la ley de Darcy (Nield y Bejan, 1992), la cual ha sido ampliamente utilizada como ecuación de transferencia de cantidad de movimiento para describir al medio poroso con flujo unidireccional (Vafai, 2000; Ingram y Pop, 1998). Esta ley revela una proporcionalidad entre la velocidad de flujo y la diferencia de presión aplicada. Esto queda expresado mediante:

$$
u_{\omega}=-\frac{K}{\mu_{\beta}} \frac{\partial p_{\omega}}{\partial x}
$$

Donde el coeficiente $K$ se le conoce como permeabilidad específica o permeabilidad intrínseca del medio poroso, el cual es independiente de la naturaleza del fluido pero dependiente de la geometría del medio y tiene unidades de longitud al cuadrado. $\frac{\partial p_{\omega}}{\partial x}$ es el gradiente de presión en la dirección del flujo y $\mu_{\beta}$ es la viscosidad cinemática del fluido. En 
el caso de flujo en una fase $K$ simplemente se conoce como permeabilidad, la expresión en su forma tridimensional para la ecuación (2.27) queda:

$$
\mathbf{v}_{\omega}=-\frac{\mathbf{K}}{\mu_{\beta}} \cdot \nabla p_{\beta}
$$

Donde la permeabilidad $\mathbf{K}$ es un tensor simétrico de segundo orden.

Sobre la postulación de la ley de Darcy se han hecho varias consideraciones tales como: ignorar los efectos inerciales, las pérdidas por fricción y se han considerado solamente con la caída de presión y las fuerzas volumétricas. Por lo tanto, esta ley es válida solamente para pequeñas velocidades de flujo (Greenkorn, 1983; Gebhart y col., 1988).

Muchos autores han utilizado conceptos estadísticos para soportar la ley de Darcy y algunos han realizado suposiciones importantes para obtener el sistema de ecuaciones de forma cerrada. No obstante, Whitaker (1986a) ha derivado la ecuación de Darcy para el caso de flujo incompresible sin considerar ninguna suposición constitutiva, en el desarrollo teórico, se asume que no existen cambios abruptos en la estructura del medio, la forma general que presenta este autor para la ecuación de Darcy es:

$$
\left\langle\mathbf{v}_{\beta}\right\rangle_{\omega}=-\frac{\mathbf{K}}{\mu_{\beta}} \cdot\left[\nabla\left\langle p_{\beta}^{\prime}\right\rangle_{\omega}-\rho_{\beta} \mathbf{g}\right]
$$

Incorporando la aproximación de Boussinesq expresada por la ecuación (2.17a) a la ecuación (2.29) la ley de Darcy en su forma dimensional queda como:

$$
0=-\frac{1}{\rho_{\beta}} \nabla^{\prime}\left\langle p_{\beta}^{\prime}\right\rangle_{\omega}^{\beta}+\mathbf{i} g \rho_{\beta} \beta\left[\left(\left\langle T_{\beta}\right\rangle_{\omega}-T_{r e f}\right)-\left(\left\langle C_{A \beta}\right\rangle_{\omega}-C_{A \beta_{r e f}}\right)\right]-\mathbf{K}^{-1} \cdot \mu_{\beta}\left\langle\mathbf{v}_{\beta}^{\prime}\right\rangle_{\omega}
$$

En la ecuación anterior no se han incluido los términos inerciales teniendo en cuenta las sugerencias realizadas por Whitaker $(1986 a, 1996)$ al llevar a cabo su desarrollo teórico. Cambiando a variables adimensionales especificadas por las ecuaciones (2.2), (2.3) y (2.13) e incluyendo los números de Rayleigh y Prandtl establecidos por las ecuaciones (2.23)-(2.24), la ecuación de Darcy queda como:

$$
0=-\operatorname{Pr} \nabla p_{\omega}+\underbrace{\mathbf{i}\left[\operatorname{Ra_{\theta }} \operatorname{Pr} \theta-\frac{R a_{\phi} P r}{L e_{\omega}} \phi\right]}_{\text {Flotación }}-\frac{\operatorname{Pr}}{D a} \mathbf{v}_{\omega}
$$


En la ecuación (2.31) se ha incluido el Número de Darcy (Da). Para el caso de un medio poroso isotrópico, el tensor se transforma en el escalar $K$, que es el término clásico que representa la permeabilidad del medio poroso.

$$
D a=\frac{K}{L_{x}^{2}}
$$

\section{b) Extensiones a la ecuación de Darcy.}

La utilización de la ley de Darcy implica que en las fronteras del sistema existe deslizamiento, ya que este modelo carece de términos difusivos que expliquen la distorsión de los perfiles de velocidad en las proximidades de una frontera sólida. Para solventar esta situación, Brinkman (1947) sugirió que a la ley de Darcy se le podría adicionar un término de segundo orden $\left(\bar{\mu} \nabla^{2} \mathbf{v}\right)$ que representa las pérdidas de energía por transporte viscoso, esta alternativa es la que comúnmente se conoce como la corrección de Brinkman a la ecuación de Darcy o simplemente como la corrección de Brinkman (Nazar y col., 2003):

$$
\nabla^{\prime}\left\langle p_{\beta}^{\prime}\right\rangle=-\mu_{\beta} \mathbf{K}^{-1} \cdot\left\langle\mathbf{v}_{\beta}^{\prime}\right\rangle_{\omega}+\underbrace{\bar{\mu} \nabla^{\prime 2}\left\langle\mathbf{v}_{\beta}^{\prime}\right\rangle_{\omega}}_{\text {Corrección de Brinkman }}
$$

En la extensión de Brinkman se cuenta con dos términos que involucran la viscosidad. El primero es el término de la ecuación de Darcy y el segundo es un término análogo al Laplaciano que aparece en la ecuación de Navier-Stokes. Brinkman también propone que $\mu=\bar{\mu}$ aunque, en un sentido riguroso, estos parámetros solamente son aproximadamente iguales (Neale y Nader, 1974).

Con la extensión de Brinkman a la ecuación de Darcy, es factible la aplicación de la condición de no deslizamiento en las paredes, aunque en la mayoría de las situaciones el efecto del transporte viscoso es despreciable, generalmente para valores del número de Darcy menores de $10^{-4}$ (Jiménez-Islas y col., 1999), No obstante, es conveniente proporcionar un soporte teórico para justificar el uso del criterio de no deslizamiento y para el acoplamiento de ecuaciones en la frontera interregional que se presentan en problemas con interfase fluidomedio poroso. Ochoa-Tapia y Whitaker $(1995 a, b)$ han propuesto una condición de salto para acoplar la ecuación de Darcy con la extensión de Brinkman a la ecuación de Navier-Stokes, lo que permite obtener un campo de velocidades continuo en todo el dominio.

A continuación se muestra la ecuación de Darcy con la corrección de Brinkman, a esta ecuación se le incluyó el término convectivo para obtener todos los términos de la ecuación de Navier-Stokes, aunque se ha demostrado que este término tiene poca importancia para flujo incompresible en medios porosos (Nield y Bejan, 1992), Jiménez y col., (1999) en sus 
resultados muestran que esté término tiene importancia para medios porosos permeables, con un valor del número de Darcy a partir de $10^{-4}$.

$$
\begin{aligned}
\underbrace{\nabla^{\prime} \cdot\left(\left\langle\mathbf{v}_{\beta}^{\prime}\right\rangle_{\eta}\left\langle\mathbf{v}_{\beta}^{\prime}\right\rangle_{\eta}\right)}_{\text {Convección }} & =-\underbrace{\frac{1}{\rho_{\beta}} \nabla^{\prime}\left\langle p_{\beta}^{\prime}\right\rangle_{\eta}^{\gamma}}_{\text {Fuerzas de presión }}+\underbrace{v_{\beta} \nabla^{\prime 2}\left\langle\mathbf{v}_{\beta}^{\prime}\right\rangle_{\eta}}_{\text {Corrección de Brinkman }} \\
& +\underbrace{\mathbf{i} g \rho_{\beta}\left[\beta_{\theta}\left(\left\langle T_{\beta}\right\rangle_{\omega}-T_{r e f}\right)-\beta_{\phi}\left(\left\langle C_{A \beta}\right\rangle_{\omega}-C_{A \beta_{\text {ref }}}\right)\right]}_{\text {Flotación }}-\frac{v}{K}\left\langle\mathbf{v}_{\beta}^{\prime}\right\rangle_{\omega}
\end{aligned}
$$

Transformando la ecuación (2.34) a variables y números adimensionales, se tiene la corrección de Brinkman a la ecuación de Darcy:

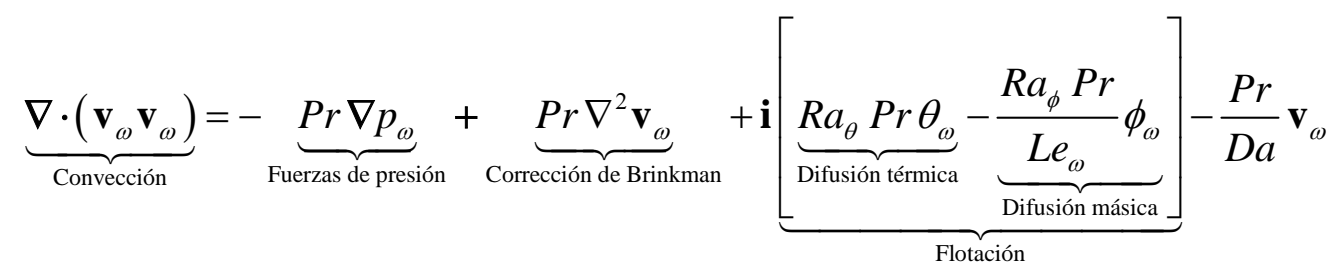

\section{CONTINUIDAD}

Sin importar el modelo utilizado para la transferencia de cantidad de movimiento en la región porosa, la ecuación de continuidad para esta región fue desarrollada previamente en su forma promediada por Ochoa-Tapia y Whitaker (1995 a,b), y su forma adimensional es:

$$
\nabla \cdot \mathbf{v}_{\lambda}=0 \quad \text { para } \lambda=\omega, \eta
$$

\subsubsection{Formulación vorticidad-vector potencial}

Como una alternativa para resolver la ecuación de cantidad de movimiento se expresa en términos de la formulación vorticidad-vector potencial en lugar de establecerlas en términos de la presión, (Raul y col., 1990; Bird y col., 2002) donde la vorticidad se define mediante:

$$
\zeta_{\lambda}=\nabla \times \mathbf{v}_{\lambda}
$$

Y las componentes del vector vorticidad en función de las componentes de la velocidad se obtienen al desarrollar la ecuación (2.37):

$$
\zeta_{X}=\frac{\partial w}{\partial Y}-\frac{\partial v}{\partial Z}
$$




$$
\begin{aligned}
& \zeta_{Y}=\frac{\partial u}{\partial Z}-\frac{\partial w}{\partial X} \\
& \zeta_{Z}=\frac{\partial v}{\partial X}-\frac{\partial u}{\partial Y}
\end{aligned}
$$

El vector velocidad en términos del vector potencial como:

$$
\mathbf{v}_{\lambda}=\nabla \times \Psi_{\lambda}
$$

Las componentes del vector velocidad en términos de las componentes del vector potencial desarrollando la ecuación (2.38) son:

$$
\begin{aligned}
& u=\frac{\partial \Psi_{Z}}{\partial Y}-\frac{\partial \Psi_{Y}}{\partial Z} \\
& v=\frac{\partial \Psi_{X}}{\partial Z}-\frac{\partial \Psi_{Z}}{\partial X} \\
& w=\frac{\partial \Psi_{Y}}{\partial X}-\frac{\partial \Psi_{X}}{\partial Y}
\end{aligned}
$$

Como resultado de la aplicación de las definiciones anteriores, la ecuación de continuidad queda automáticamente satisfecha ya que la divergencia del rotacional de cualquier vector es cero.

Aplicando las ecuaciones (2.37) y (2.38) a cada una de las ecuaciones de transferencia de cantidad de movimiento, se tendrá una ecuación para el vector potencial y otra para el vector vorticidad, omitiendo el desarrollo las ecuaciones serán:

Región fluida: Navier-Stokes

$$
\begin{gathered}
\nabla^{2} \Psi_{\eta}=-\zeta_{\eta} \\
\underbrace{\nabla \times\left(\zeta_{\eta} \times \mathbf{v}_{\eta}\right)}_{\text {Convección }}=\underbrace{\operatorname{Pr} \nabla^{2} \zeta_{\eta}}_{\text {Fuerzas viscosas }}+\underbrace{\left(R a_{\theta} \operatorname{Pr}\right) \nabla \times\left(\mathbf{i} \theta_{\omega}\right)}_{\text {Dlotación }}-\underbrace{\left(\frac{R a_{\phi} P r}{L e_{i}}\right) \nabla \times\left(\mathbf{i} \phi_{\omega}\right)}_{\text {Difusión térmica }}
\end{gathered}
$$

Medio poroso: Darcy

$$
\begin{gathered}
\nabla^{2} \Psi_{\omega}=-\zeta_{\omega} \\
0=\underbrace{\left(R a_{\theta} P r\right) \nabla \times(\mathbf{i} \theta)}_{\text {Difusión térmica }}-\underbrace{\left(\frac{R a_{\phi} P r}{L e_{\omega}}\right) \nabla \times(\mathbf{i} \phi)}_{\text {Difusión másica }}-\left(\frac{P r}{D a}\right) \zeta_{\omega}
\end{gathered}
$$


Medio poroso: Corrección de Brinkman

$$
\begin{aligned}
& \nabla^{2} \Psi_{\omega}=-\zeta_{\omega} \\
& \underbrace{\nabla \times\left(\zeta_{\omega} \times \mathbf{v}_{\omega}\right)}_{\text {Convección }}=\underbrace{\operatorname{Pr} \nabla^{2} \zeta_{\omega}}_{\text {Corrección de Brinkman }}+\underbrace{\left(\operatorname{Ra} a_{\theta} \operatorname{Pr}\right) \nabla \times\left(\mathbf{i} \theta_{\omega}\right)}_{\text {Flotación }}-\underbrace{\left(\frac{\operatorname{Ra} a_{\phi} P r}{L e_{\omega}}\right) \nabla \times\left(\mathbf{i} \phi_{\omega}\right)}_{\text {Difusión térmica }}-\left(\frac{\operatorname{Pr}}{D a}\right) \zeta_{\omega}
\end{aligned}
$$

Las ecuaciones para la transferencia de cantidad de movimiento en términos del vector vorticidad también se pueden expresar en función del número de flotación $(N)$, el cual relaciona los términos de flotación debido al transporte de energía con respecto al transporte de masa, quedando para cada uno de los modelos:

Navier-Stokes

$$
\nabla \times\left(\zeta_{\eta} \times \mathbf{v}_{\eta}\right)=\operatorname{Pr} \nabla^{2} \zeta_{\eta}+\left(R a_{\theta} \operatorname{Pr}\right)\left[\nabla \times\left(\mathbf{i} \theta_{\eta}\right)-N \nabla \times\left(\mathbf{i} \phi_{\eta}\right)\right]
$$

Darcy:

$$
0=\left(R a_{\theta} \operatorname{Pr}\right)\left[\nabla \times\left(\mathbf{i} \theta_{\eta}\right)-N \nabla \times\left(\mathbf{i} \phi_{\eta}\right)\right]-\left(\frac{P r}{D a}\right) \zeta_{\omega}
$$

Brinkman:

$\nabla \times\left(\zeta_{\omega} \times \mathbf{v}_{\omega}\right)=\operatorname{Pr} \nabla^{2} \zeta_{\omega}+\left(R a_{\theta} \operatorname{Pr}\right)\left[\nabla \times\left(\mathbf{i} \theta_{\omega}\right)-N \nabla \times\left(\mathbf{i} \phi_{\omega}\right)\right]-\left(\frac{P r}{D a}\right) \zeta_{\omega}$

Donde el número de flotación está definido mediante:

$$
N=\frac{\beta_{\theta} \Delta \theta}{\beta_{\phi} \Delta \phi}=\frac{R a_{\theta}}{R a_{\phi}}
$$

\subsubsection{Condiciones de frontera y ecuación en la interregión para la transferencia de cantidad de movimiento.}

Las condiciones de frontera para resolver las ecuaciones de cantidad de movimiento se escriben en términos de las variables vorticidad-vector potencial.

\section{VECTOR POTENCIAL:}

Las condiciones de frontera para el vector potencial fueron discutidas por varios autores (Leonardi, 1984; Singh y col., 1993; Hirasaki y Hellums, 1968; 1970; Aziz y Hellums, 1985, entre otros) llegando a la conclusión que en las superficies las componentes del vector 
potencial deben de satisfacer el no deslizamiento y la no penetración, esto implica que para la derivada normal del componete normal es cero, mientras que las componentes tangenciales son cero:

$$
\begin{gathered}
\frac{\partial \Psi_{n}}{\partial n}=0 \\
\Psi_{t}=0
\end{gathered}
$$

Donde “ $n$ ” indica la componente normal y “ $t$ ” cualquiera de las componentes tangenciales.

\section{INTERREGIÓN}

Cuando se utiliza la corrección de Brinkman, a la ecuación de Darcy, en la interregión se tienen las siguientes condiciones para el vector potencial:

$$
\begin{gathered}
\Psi_{\eta}=\Psi_{\omega} \\
\frac{\partial \Psi_{\eta}}{\partial x}=\frac{\partial \Psi_{\omega}}{\partial x}
\end{gathered}
$$

\section{VORTICIDAD}

Para obtener las condiciones de frontera para la vorticidad se utilizó una aproximación sugerida por Mallinson y De Vahl Davis (1973, 1997) y utilizadas posteriormente por Leonardi (1984), teniéndose las siguientes relaciones:

$$
\begin{aligned}
& \zeta_{n}=0 \\
& \zeta_{t_{1}}=-\frac{\partial^{2} \Psi_{t_{1}}}{\partial n^{2}} \\
& \zeta_{t_{2}}=-\frac{\partial^{2} \Psi_{t_{2}}}{\partial n^{2}}
\end{aligned}
$$

Donde $n$ indica la coordenada cartesiana normal a la pared y t1 y t2 son las restantes coordenadas.

Las condiciones en la interregión quedan expresadas por:

$$
\begin{aligned}
& \zeta_{f}=\zeta_{p} \\
& \frac{\partial \zeta_{f}}{\partial x}=\frac{\partial \zeta_{p}}{\partial x}
\end{aligned}
$$




\section{a) Condición de Beavers y Joseph para la transferencia de cantidad de movimiento en la interregión.}

Si el medio poroso se encuentra adyacente a una región fluida con la cual está saturada el medio poroso (ver Figura 2.2), entonces de acuerdo con Beavers y Joseph (1967), la condición de frontera en la interregión puede expresarse mediante la siguiente relación empírica para el caso bidimensional:

$$
\frac{\partial u_{\omega}}{\partial X}=\frac{\alpha_{b j}}{\sqrt{K}}\left(u_{\eta}-u_{\omega}\right)
$$

De acuerdo a la Figura 2.2, $u_{\omega}$ se evalúa a una distancia relativamente pequeña del plano interregional, de tal forma que existe una pequeña zona dentro del medio poroso donde tiene lugar la transición de la velocidad. La cantidad $\alpha_{b j}$ es un parámetro adimensional que es independiente de la viscosidad del fluido, pero es dependiente de las propiedades del material que caracteriza a la región permeable. En sus experimentos Beavers y Joseph (1967) han determinado valores de $\alpha_{b j}$ para diferentes materiales.

En el presente trabajo se aplicó la expresión generalizada utilizada por Ochoa-Tapia y Whitaker (1995 a) para las condiciones de Beavers y Joseph expresada mediante la ecuación (2.58); en ella se consideran los términos que involucran las derivadas de las componentes del vector velocidad :

$$
-\mathbf{t}_{\omega \eta} \cdot \frac{\partial \mathbf{v}_{\omega}}{\partial X}=\frac{\alpha_{b j}}{\sqrt{D a}} \mathbf{t}_{\omega \eta} \cdot\left(\mathbf{v}_{\omega}-\mathbf{v}_{\eta}\right) \quad \text { para } \mathbf{t}_{\omega \eta}=\mathbf{j}, \mathbf{k}
$$

Estas condiciones se expresan en términos de las componentes de la vorticidad en función del vector potencial mediante la aplicación de las ecuaciones (3.37 a)-(3.37 c):

$$
\begin{aligned}
& \zeta_{X \eta}=\left(\frac{\partial \Psi_{Z}}{\partial Y}-\frac{\partial \Psi_{Y}}{\partial Z}\right)_{\omega}-\left(\frac{\partial \Psi_{Z}}{\partial Y}-\frac{\partial \Psi_{Y}}{\partial Z}\right)_{\eta}+\left(\frac{\partial w}{\partial Y}-\frac{\partial v}{\partial Z}\right)_{\eta} \\
& \zeta_{Y \eta}=\frac{\alpha_{b j}}{\sqrt{D a}}\left[\left(\frac{\partial \Psi_{Y}}{\partial X}-\frac{\partial \Psi_{X}}{\partial Y}\right)_{\eta}-\left(\frac{\partial \Psi_{Y}}{\partial X}-\frac{\partial \Psi_{X}}{\partial Y}\right)_{\omega}\right]+\frac{\partial u_{\eta}}{\partial Z} \\
& \zeta_{Z \eta}=-\frac{\alpha_{b j}}{\sqrt{D a}}\left[\left(\frac{\partial \Psi_{X}}{\partial Z}-\frac{\partial \Psi_{Z}}{\partial X}\right)_{\eta}-\left(\frac{\partial \Psi_{X}}{\partial Z}-\frac{\partial \Psi_{Z}}{\partial X}\right)_{\omega}\right]-\frac{\partial u_{\eta}}{\partial Y}
\end{aligned}
$$




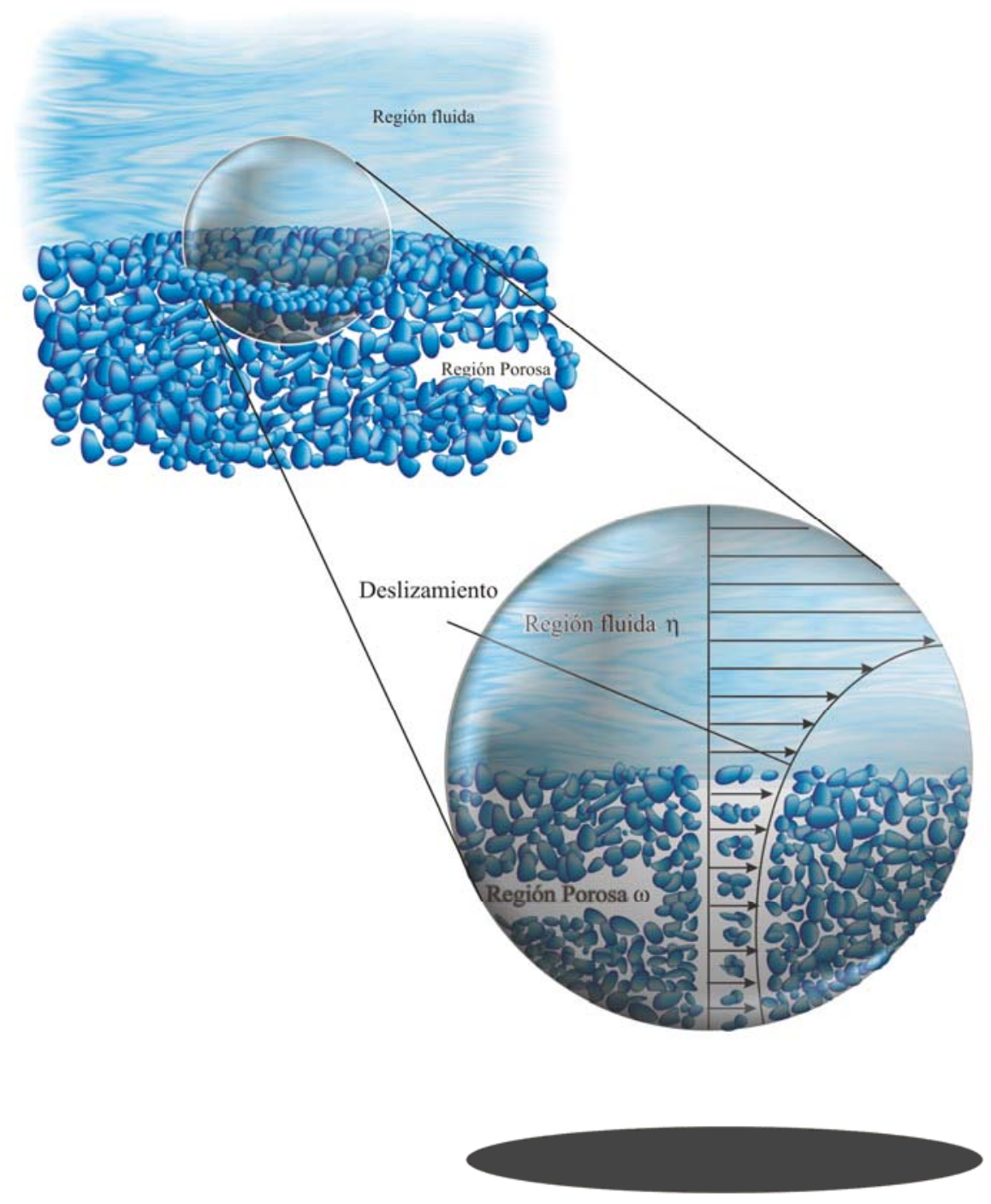

Figura 2.2.- Condición interregional de Beavers y Joseph. 
Los términos que involucran las derivadas de las componentes del vector velocidad en las ecuaciones (2.59)-(2.61) se pueden evalúan en una iteración anterior en el método numérico, o bien utilizar la definición de las componentes de la velocidad en términos del vector potencial especificadas por las ecuaciones (2.38 a)-(2.38 c) para tener las siguientes condiciones interregionales para los componentes de la vorticidad:

$$
\begin{aligned}
& \zeta_{X \eta}=\left(\frac{\partial \Psi_{Z}}{\partial Y}-\frac{\partial \Psi_{Y}}{\partial Z}\right)_{\omega}-\left(\frac{\partial \Psi_{Z}}{\partial Y}-\frac{\partial \Psi_{Y}}{\partial Z}\right)_{\eta}+\left(\frac{\partial^{2} \Psi_{Y}}{\partial X \partial Y}-\frac{\partial^{2} \Psi_{X}}{\partial Y^{2}}\right)_{\eta}-\left(\frac{\partial^{2} \Psi_{X}}{\partial Z^{2}}-\frac{\partial^{2} \Psi_{Z}}{\partial Z \partial X}\right)_{\eta} \\
& \zeta_{Y \eta}=\frac{\alpha_{b j}}{\sqrt{D a}}\left[\left(\frac{\partial \Psi_{Y}}{\partial X}-\frac{\partial \Psi_{X}}{\partial Y}\right)_{\eta}-\left(\frac{\partial \Psi_{Y}}{\partial X}-\frac{\partial \Psi_{X}}{\partial Y}\right)_{\omega}\right]+\left(\frac{\partial^{2} \Psi_{Z}}{\partial Z \partial Y}-\frac{\partial^{2} \Psi_{Y}}{\partial Z^{2}}\right)_{\eta} \\
& \zeta_{Z \eta}=-\frac{\alpha_{b j}}{\sqrt{D a}}\left[\left(\frac{\partial \Psi_{X}}{\partial Z}-\frac{\partial \Psi_{Z}}{\partial X}\right)_{\eta}-\left(\frac{\partial \Psi_{X}}{\partial Z}-\frac{\partial \Psi_{Z}}{\partial X}\right)_{\omega}\right]-\left(\frac{\partial^{2} \Psi_{Z}}{\partial Y^{2}}-\frac{\partial^{2} \Psi_{Y}}{\partial Y \partial Z}\right)_{\eta}
\end{aligned}
$$

\subsection{Resumen de ecuaciones para los dos modelos.}

En las secciones anteriores se han desarrollado cada una de las ecuaciones en la región fluida, y para el caso del medio poroso se ha puesto particular atención a la ecuación de cantidad de movimiento, considerando dos modelos, estos son: la ecuación de Darcy y su acoplamiento en la interregión mediante la condición de Beavers y Joseph (Darcy-Beavers y Joseph) y el segundo método que considera la corrección de Brinkman a la ecuación de Darcy para la ecuación de cantidad de movimiento en la región porosa (Brinkman), a continuación se presenta un resumen de los dos modelos:

\section{Brinkman}

REGIÓN FLUIDA

$$
\begin{gathered}
\nabla^{2} \Psi_{\eta}=-\zeta_{\eta} \\
\nabla \times\left(\zeta_{\eta} \times \mathbf{v}_{\eta}\right)=\operatorname{Pr} \nabla^{2} \zeta_{\eta}+\left(R a_{\theta} \operatorname{Pr}\right) \nabla \times\left(\mathbf{i} \theta_{\eta}\right)-\left(\frac{R a_{\phi} P r}{L e}\right) \nabla \times\left(\mathbf{i} \phi_{\eta}\right) \\
\nabla \cdot\left(\mathbf{v}_{\eta} \theta_{\eta}\right)=\nabla^{2} \theta_{\eta} \\
\mathbf{v}_{\eta} \cdot \nabla \phi_{\eta}=\frac{1}{L e} \nabla^{2} \phi_{\eta}
\end{gathered}
$$

REGIÓN POROSA.

$$
\begin{gathered}
\nabla^{2} \Psi_{\omega}=-\zeta_{\omega} \\
\nabla \times\left(\zeta_{\omega} \times \mathbf{v}_{\omega}\right)=\operatorname{Pr} \nabla^{2} \zeta_{\omega}+\left(R a_{\theta} \operatorname{Pr}\right) \nabla \times\left(\mathbf{i} \theta_{\omega}\right)-\left(\frac{R a_{\phi} P r}{L e}\right) \nabla \times\left(\mathbf{i} \phi_{\omega}\right)-\left(\frac{P r}{D a}\right) \zeta_{\omega} \\
\nabla \cdot\left(\mathbf{v}_{\omega} \theta_{\omega}\right)=K_{\omega} \nabla^{2} \theta_{\omega}
\end{gathered}
$$




$$
\mathbf{v}_{\omega} \cdot \nabla \phi_{\omega}=\frac{1}{L e} \nabla^{2} \phi_{\omega}
$$

\section{Condiciones de frontera}

TEMPERATURA

$$
\begin{array}{ll}
\left(\theta_{\lambda}\right)_{b}=a & \text { Dirichlet } \\
\left(\frac{d \theta_{\lambda}}{d n}\right)_{b}=a & \text { Newman } \\
\left(\frac{d \theta_{\lambda}}{d n}\right)_{b}+a\left(\theta_{\lambda}\right)_{b}=c & \text { Cauchy } \\
\theta_{\omega}=\theta_{\eta} & \\
-\mathbf{n}_{\omega \eta} \cdot K_{\omega} \nabla \theta_{\omega}=-\mathbf{n}_{\omega \eta} \cdot \nabla \theta_{\eta}
\end{array}
$$

CONCENTRACIÓN

$$
\begin{array}{ll}
\left(\phi_{\lambda}\right)_{b}=a & \text { Dirichlet } \\
\left(\frac{d \phi_{\lambda}}{d n}\right)_{b}=a & \text { Newman } \\
\left(\frac{d \phi_{\lambda}}{d n}\right)_{b}+a\left(\phi_{\lambda}\right)_{b}=c & \text { Cauchy } \\
\phi_{\omega}=\phi_{\eta} & \\
-\mathbf{n}_{\omega \eta} \cdot\left[\mathbf{D}_{\omega}^{*} \cdot \nabla \phi_{\omega}-\mathrm{D}_{\eta} \nabla \phi_{\eta}\right]=0
\end{array}
$$

VECTOR POTENCIAL:

$$
\begin{gathered}
\frac{\partial \Psi_{n}}{\partial n}=0 \\
\Psi_{t}=0
\end{gathered}
$$

Las condiciones interregionales:

$$
\begin{aligned}
\Psi_{f} & =\Psi_{p} \\
\frac{\partial \Psi_{f}}{\partial x} & =\frac{\partial \Psi_{p}}{\partial x}
\end{aligned}
$$

VORTICIDAD

$$
\begin{aligned}
& \zeta_{n}=0 \\
& \zeta_{t}=-\frac{\partial^{2} \Psi_{t}}{\partial n^{2}}
\end{aligned}
$$

y en la interregión:

$$
\begin{gathered}
\zeta_{f}=\zeta_{p} \\
\frac{\partial \zeta_{f}}{\partial x}=\frac{\partial \zeta_{p}}{\partial x}
\end{gathered}
$$




\section{Darcy-Beavers y Joseph.}

REGIÓN FLUIDA

$$
\begin{gathered}
\nabla^{2} \Psi_{\eta}=-\zeta_{\eta} \\
\nabla \times\left(\zeta_{\eta} \times \mathbf{v}_{\eta}\right)=\operatorname{Pr} \nabla^{2} \zeta_{\eta}+\left(R a_{\theta} \operatorname{Pr}\right) \nabla \times\left(\mathbf{i} \theta_{\eta}\right)-\left(\frac{R a_{\phi} P r}{L e}\right) \nabla \times\left(\mathbf{i} \phi_{\eta}\right) \\
\nabla \cdot\left(\mathbf{v}_{\eta} \theta_{\eta}\right)=\nabla^{2} \theta_{\eta} \\
\mathbf{v}_{\eta} \cdot \nabla \phi_{\eta}=\frac{1}{L e} \nabla^{2} \phi_{\eta}
\end{gathered}
$$

REGIÓN POROSA

$$
\begin{gathered}
\nabla^{2} \Psi_{\omega}=-\zeta_{\omega} \\
0=\left(R a_{\theta} P r\right) \nabla \times(\mathbf{i} \theta)-\left(\frac{R a_{\phi} P r}{L e}\right) \nabla \times(\mathbf{i} \phi)-\left(\frac{P r}{D a}\right) \zeta_{\omega} \\
\nabla \cdot\left(\mathbf{v}_{\eta} \theta_{\eta}\right)=\nabla^{2} \theta_{\eta} \\
\mathbf{v}_{\omega} \cdot \nabla \phi_{\omega}=\frac{1}{L e} \nabla^{2} \phi_{\omega}
\end{gathered}
$$

\section{Condiciones de frontera}

TEMPERATURA

$$
\begin{array}{ll}
\left(\theta_{\lambda}\right)_{b}=a & \text { Dirichlet } \\
\left(\frac{d \theta_{\lambda}}{d n}\right)_{b}=a & \text { Newman } \\
\left(\frac{d \theta_{\lambda}}{d n}\right)_{b}+a\left(\theta_{\lambda}\right)_{b}=c & \text { Cauchy } \\
\theta_{\omega}=\theta_{\eta} & \\
-\mathbf{n}_{\omega \eta} \cdot K_{\omega} \nabla \theta_{\omega}=-\mathbf{n}_{\omega \eta} \cdot \nabla \theta_{\eta}
\end{array}
$$

CONCENTRACIÓN$$
\left(\phi_{\lambda}\right)_{b}=a \quad \text { Dirichlet }
$$$$
\left(\frac{d \phi_{\lambda}}{d n}\right)_{b}=a \quad \text { Newman }
$$$$
\left(\frac{d \phi_{\lambda}}{d n}\right)_{b}+a\left(\phi_{\lambda}\right)_{b}=c \quad \text { Cauchy }
$$$$
\phi_{\omega}=\phi_{\eta}
$$$$
-\mathbf{n}_{\omega \eta} \cdot\left[\mathbf{D}_{\omega}^{*} \cdot \nabla \phi_{\omega}-\mathbf{D}_{\eta} \nabla \phi_{\eta}\right]=0
$$ 
Condiciones de frontera para el vector vorticidad

$$
\begin{aligned}
& \zeta_{n}=0 \\
& \zeta_{t}=-\frac{\partial^{2} \Psi_{t}}{\partial n^{2}}
\end{aligned}
$$

Condiciones interregionales para el vector potencial

$$
\begin{aligned}
& \zeta_{X \eta}=\left(\frac{\partial \Psi_{Z}}{\partial Y}-\frac{\partial \Psi_{Y}}{\partial Z}\right)_{\omega}-\left(\frac{\partial \Psi_{Z}}{\partial Y}-\frac{\partial \Psi_{Y}}{\partial Z}\right)_{\eta}+\left(\frac{\partial^{2} \Psi_{Y}}{\partial X \partial Y}-\frac{\partial^{2} \Psi_{X}}{\partial Y^{2}}\right)_{\eta}-\left(\frac{\partial^{2} \Psi_{X}}{\partial Z^{2}}-\frac{\partial^{2} \Psi_{Z}}{\partial Z \partial X}\right)_{\eta} \\
& \zeta_{Y \eta}=\frac{\alpha_{b j}}{\sqrt{D a}}\left[\left(\frac{\partial \Psi_{Y}}{\partial X}-\frac{\partial \Psi_{X}}{\partial Y}\right)_{\eta}-\left(\frac{\partial \Psi_{Y}}{\partial X}-\frac{\partial \Psi_{X}}{\partial Y}\right)_{\omega}\right]+\left(\frac{\partial^{2} \Psi_{Z}}{\partial Z \partial Y}-\frac{\partial^{2} \Psi_{Y}}{\partial Z^{2}}\right)_{\eta} \\
& \zeta_{Z \eta}=-\frac{\alpha_{b j}}{\sqrt{D a}}\left[\left(\frac{\partial \Psi_{X}}{\partial Z}-\frac{\partial \Psi_{Z}}{\partial X}\right)_{\eta}-\left(\frac{\partial \Psi_{X}}{\partial Z}-\frac{\partial \Psi_{Z}}{\partial X}\right)_{\omega}\right]-\left(\frac{\partial^{2} \Psi_{Z}}{\partial Y^{2}}-\frac{\partial^{2} \Psi_{Y}}{\partial Y \partial Z}\right)_{\eta}
\end{aligned}
$$

La discretización de estos modelos matemáticos, su planteamiento numérico y solución se presentan en el siguiente capítulo. 


\section{Capítulo 3}

\section{Procedimiento Numérico.}

En el presente capítulo se muestra el procedimiento numérico utilizado para la solución de los sistemas de ecuaciones diferenciales. El procedimiento incluye la forma para discretizar las ecuaciones, el método para resolver el sistema resultante y la secuencia jerárquica de ejecución adoptada.

De las dos regiones que constituyen la cavidad, la fluida está gobernada por el mismo sistema de ecuaciones para ambos modelos, es decir, en el caso de la ecuación de cantidad de movimiento, la ecuación de Navier-Stokes, por lo que el método utilizado es el mismo. Para el caso de la región porosa se tomará el modelo que utiliza la corrección de Brinkman a la ecuación de Darcy para mostrar la forma de discretizar las ecuaciones y la secuencia de evaluación. Los detalles que son exclusivos del modelo que utiliza la ecuación de Darcy en la región porosa y el acoplamiento interregional con las condiciones de Beavers y Joseph (1967) se mostrarán en una sección independiente. Para el caso del modelo en dos dimensiones, la formulación matemática y la parte numérica se presentan en el Apéndice C.

El sistema de ecuaciones diferenciales no-lineales en términos de las variables: temperatura $(\theta)$, concentración $(\phi)$, vector verticidad $(\zeta)$ y vector potencial $(\Psi)$ se resolvió usando un procedimiento similar al presentado por Mallinson y De Vahl Davis (1973) para convección natural en flujos de una sola fase y utilizada posteriormente por Singh y col., (1993). En este método las ecuaciones gobernantes son transformadas a la forma parabólica por la adición del término transitorio, éstas posteriormente se representan en forma discretizada utilizando diferencias finitas. Las ecuaciones discretizadas son resueltas utilizando el método de integración implícita de dirección alternada (ADI) propuesto por Peaceman y Rachford (1955) y aplicado en varios trabajos recientes (Bubnovich y col., 2002; Provisky, 2002; Dai y col., 2000), los detalles de cada uno de estos puntos se muestran en las secciones siguientes. 


\subsection{Ecuaciones Elípticas.}

El hecho de que las ecuaciones gobernantes de flujo multidimensional sean acopladas y elípticas ocasiona una gran complejidad matemática. Para el problema que se presenta se tendrían que resolver simultáneamente para cada una de las dos regiones: 3 ecuaciones diferenciales parciales para el vector vorticidad, 3 ecuaciones para el vector potencial, 1 ecuación para la temperatura y 1 ecuación para la concentración, dando un total de 16 ecuaciones diferenciales parciales por cada región, cada sistema de ecuaciones incluye sus correspondientes condiciones de frontera e interregionales, lo anterior sugiere que la solución del sistema de ecuaciones debe obtenerse mediante un procedimiento numérico. Aún con esta simplificación, las formas discretizadas de las ecuaciones deben de ser resueltas simultáneamente por un método que requiere un procedimiento doblemente iterativo. Sin embargo, una revisión bibliográfica (Mallinson y De Vahl Davis, 1973, 1977; Peaceman y Rachford, 1955) recomienda el método de integración implícita de dirección alternada (ADI); La aplicación específica de este método se encuentra en la solución de ecuaciones diferenciales no-lineales parabólicas. El método indica que para su utilización se requiere de la transformación de las ecuaciones gobernantes (elípticas), esto se logrará al utilizar el método del falso estado transitorio, el cual consiste en la adición de la dependencia artificial del tiempo con el fin de encontrar el estado estacionario, esto es, cuando la variación con respecto al tiempo es significantemente pequeña se alcanza la convergencia; una explicación detallada se encuentra en: Behnia y col. (1996) y Mallinson y De Vahl Davis (1973) y los puntos específicos para el sistema en particular se desarrollan a partir de la sección 3.3.

\subsection{Ecuaciones Parabólicas.}

La transformación de las ecuaciones a la forma parabólica para la aplicación del método del falso estado transitorio se puede realizar para cada una de las ecuaciones gobernantes y en cada región mediante la adición artificial del tiempo, esto incluye la incorporación de un parámetro que ayuda a la estabilidad, así por ejemplo, para el modelo que considera la corrección de Brinkman a la ecuación de Darcy se puede expresar el sistema de ecuaciones de la siguiente manera:

\section{Región fluida $\eta$}

- $\quad$ Cantidad de movimiento: Vorticidad-vector potencial

Vector potencial

$$
\frac{1}{\left(\alpha_{\Psi}\right)_{\eta}} \frac{\partial \Psi}{\partial t}=\zeta_{\eta}+\nabla^{2} \Psi_{\eta}
$$




$$
\begin{gathered}
\text { Vector vorticidad } \\
\frac{1}{\left(\alpha_{\zeta}\right)_{\eta}} \frac{\partial \zeta}{\partial t}=-\nabla \times\left(\zeta_{\eta} \times \mathbf{v}_{\eta}\right)+\operatorname{Pr} \nabla^{2} \zeta_{\eta}+\left(R a_{\theta} \operatorname{Pr}\right) \nabla \times\left(\mathbf{i} \theta_{\eta}\right)-\left(\frac{R a_{\phi} P r}{L e}\right) \nabla \times\left(\mathbf{i} \phi_{\eta}\right)
\end{gathered}
$$

- Energía

$$
\frac{1}{\left(\alpha_{\theta}\right)_{\eta}} \frac{\partial \theta}{\partial t}=\nabla^{2} \theta_{\eta}-\nabla \cdot\left(\mathbf{v}_{\eta} \theta_{\eta}\right)
$$

- $\quad$ Masa

$$
\frac{1}{\left(\alpha_{\phi}\right)_{\eta}} \frac{\partial \phi}{\partial t}=\frac{1}{L e} \nabla^{2} \phi_{\eta}-\mathbf{v}_{\eta} \cdot \nabla \phi_{\eta}
$$

Región porosa $\omega$

- $\quad$ Cantidad de movimiento: Vorticidad-vector potencial

Vector potencial

$$
\frac{1}{\left(\alpha_{\Psi}\right)_{\omega}} \frac{\partial \Psi}{\partial t}=\zeta_{\omega}+\nabla^{2} \Psi_{\omega}
$$

Vector vorticidad

$$
\begin{aligned}
& \frac{1}{\left(\alpha_{\zeta}\right)_{\omega}} \frac{\partial \zeta}{\partial t}=-\nabla \times\left(\zeta_{\omega} \times \mathbf{v}_{\omega}\right)+\operatorname{Pr} \nabla^{2} \zeta_{\omega} \\
&+\left(R a_{\theta} \operatorname{Pr}\right) \nabla \times\left(\mathbf{i} \theta_{\omega}\right)-\left(\frac{R a_{\phi} P r}{L e}\right) \nabla \times\left(\mathbf{i} \phi_{\omega}\right)-\left(\frac{P r}{D a}\right) \zeta_{\omega}
\end{aligned}
$$

- Energía

$$
\frac{1}{\left(\alpha_{\theta}\right)_{\omega}} \frac{\partial \theta}{\partial t}=K_{\omega} \nabla^{2} \theta_{\omega}-\nabla \cdot\left(\mathbf{v}_{\omega} \theta_{\omega}\right)
$$

- Masa

$$
\frac{1}{\left(\alpha_{\phi}\right)_{\omega}} \frac{\partial \phi}{\partial t}=\frac{1}{L e} \nabla^{2} \phi_{\omega}-\mathbf{v}_{\omega} \cdot \nabla \phi_{\omega}
$$


En este conjunto de ecuaciones $\left(\alpha_{\zeta}\right)_{\eta},\left(\alpha_{\Psi}\right)_{\eta},\left(\alpha_{\theta}\right)_{\eta},\left(\alpha_{\phi}\right)_{\eta},\left(\alpha_{\zeta}\right)_{\omega},\left(\alpha_{\Psi}\right)_{\omega},\left(\alpha_{\theta}\right)_{\omega} \mathrm{y}$ $\left(\alpha_{\phi}\right)_{\omega}$ representan parámetros de magnitud positiva propios del método del falso estado transitorio, la buena elección de valores para estos parámetros contribuye a incrementar o disminuir la velocidad de convergencia de las ecuaciones y la estabilidad del método. Los valores de los parámetros que incrementan la velocidad de convergencia dependen de varios factores, entre los cuales se pueden mencionar: el tamaño de la red, el incremento del tiempo artificial, las condiciones de frontera en las paredes, el tipo de espaciamiento (constante o variable), entre otros. Varios autores han sugerido diferentes valores para los coeficientes, coincidiendo en establecer un valor relativamente pequeño para la ecuación de vorticidad en comparación con los coeficientes de las ecuaciones restantes, en la Tabla 3.1 se muestran los valores utilizados por Leonardi (1984) para una cavidad fluida y por Singh y col. (1993) para un sistema fluido-medio poroso. Cabe hacer la aclaración que estos valores se establecieron al utilizar un modelo que considera la corrección de Brinkman como ecuación de transferencia de cantidad de movimiento en la región porosa.

\begin{tabular}{|c|c|c|c|}
\hline Autor & Ecuación & Fluido & Medio poroso \\
\hline \hline \multirow{2}{*}{$\begin{array}{c}\text { Leonardi } \\
1984\end{array}$} & Vorticidad & 0.05 & $\ldots$ \\
\cline { 2 - 4 } & Energía & 1 & $\ldots$ \\
\hline \multirow{2}{*}{$\begin{array}{c}\text { Singh y col. } \\
1993\end{array}$} & Vorticidad & 0.1 & 2.5 \\
\cline { 2 - 4 } & Energía & 5 & 5 \\
\hline
\end{tabular}

Tabla 3.1 Coeficientes utilizados para el método del falso estado transitorio

De acuerdo a la revisión bibliográfica realizada no existe una dependencia específica para establecer los valores de dichas constantes. Por otra parte, dentro de los objetivos del presente trabajo no se tiene contemplada la optimización del método numérico, por lo que los valores de los parámetros anteriores se establecerán tomando las recomendaciones de Singh $y$ col. (1993), y en algunas pruebas se utilizará el conjunto de valores que garanticen la estabilidad y convergencia del método (Valencia, 1993).

\subsection{Diferencias finitas.}

El sistema considerado para las pruebas numéricas se encuentra en coordenadas cartesianas y con una interregión plana y horizontal, razones por las cuales se eligió la discretización de las ecuaciones mediante diferencias finitas. En la discretización se tiene cuidado de utilizar incrementos variables para considerar los cambios bruscos que ocurren en las fronteras o en la interregión. 
Para la generación de los espaciamientos variables se optó por la aproximación a la distribución normal mediante el polinomio de Newton (Brockwell y Davis, 2002), utilizando la siguiente expresión para el espaciamiento k+1:

$$
\Delta_{k+1}=\left(\begin{array}{l}
n \\
k
\end{array}\right) a^{n-k} b^{k}
$$

Donde $\mathrm{a}=\mathrm{b}=0.5$ y $n$ es el número de espaciamientos

La forma simétrica para el eje $X$ se muestra en la Figura 3.1 a). Los espaciamientos se incluyen en un archivo de datos para ser leídos por el programa computacional, de tal forma que pueden asignar arbitrariamente con alguna distribución específica.

El espaciamiento en la dirección $X$, por ser la dirección que considera la interregión, está compuesta por dos distribuciones, una por cada región (ver Figura 2.1 ), los detalles de la discretización con espaciamiento variable se puede consultar en el Apéndice D, dicha variación de los incrementos en la malla computacional se puede observar en la Figura 3.1 b).

A continuación se mostrará la forma en que se llevó a cabo la discretización de las ecuaciones y la secuencia utilizada en el método de integración implícita de dirección alternada (ADI).

\subsubsection{Discretización de la ecuación de energía.}

Con la adimensionalización de la temperatura especificada por las ecuaciones (3.3) y (3.7) se obtienen las ecuaciones de energía para cada región. Cualquiera de estas ecuaciones puede ser representada mediante la siguiente ecuación:

$$
\frac{1}{\left(\alpha_{\theta}\right)_{\lambda}} \frac{\partial \theta}{\partial t}=\mathbf{G} \nabla^{2} \theta_{\lambda}-F \nabla \cdot\left(\mathbf{v}_{\lambda} \theta_{\lambda}\right)
$$

Donde los coeficientes del Laplaciano y Divergencia G y $F$ adquieren los siguientes valores para cada una de las regiones

\begin{tabular}{|l|c|c|}
\cline { 2 - 3 } \multicolumn{1}{c|}{} & Fluido & Medio poroso \\
\hline$G_{i}$ & 1 & $K_{\omega}$ \\
\hline$F$ & 1 & 1 \\
\hline
\end{tabular}

Tabla 3.2 Coeficientes utilizados en la ecuación de energía. 


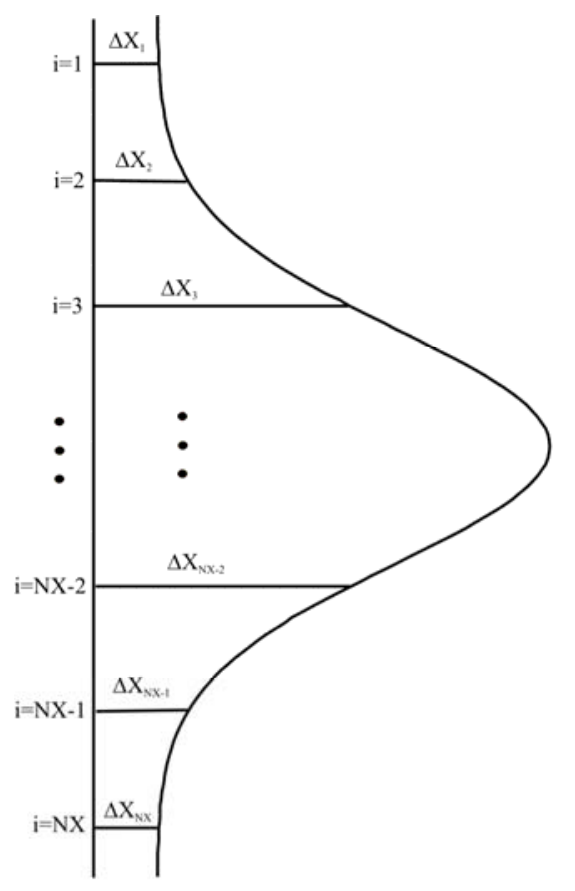

a)

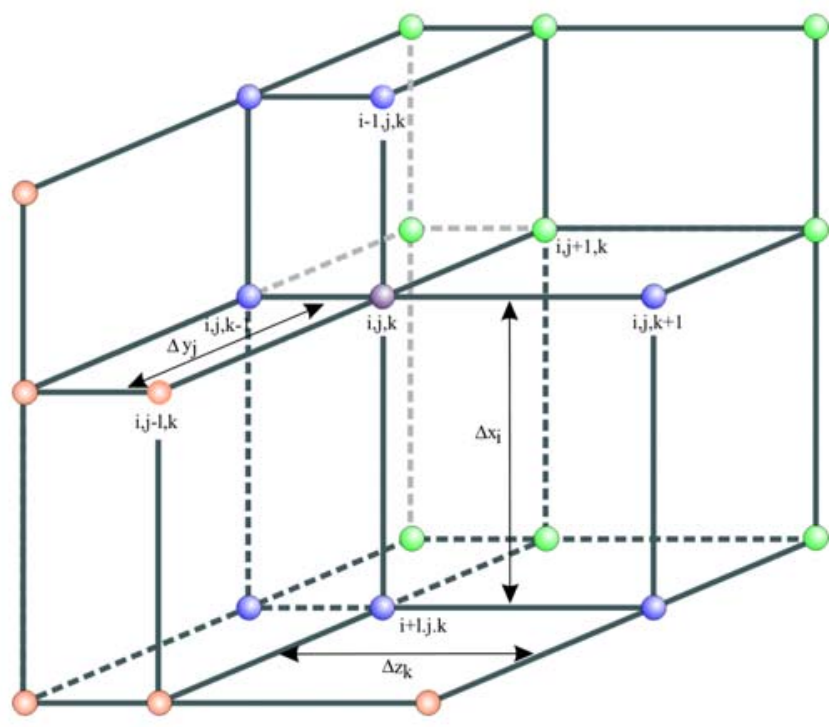

b)

Figura 3.1.- Asignación del espaciamiento variable y celda computacional para el sistema tridimensional. 
Desarrollando el Laplaciano y la Divergencia se obtiene:

$$
\frac{1}{\left(\alpha_{\theta}\right)_{\lambda}} \frac{\partial \theta_{\lambda}}{\partial t}+F\left[\frac{\partial\left(u_{\lambda} \theta_{\lambda}\right)}{\partial X}+\frac{\partial\left(v_{\lambda} \theta_{\lambda}\right)}{\partial Y}+\frac{\partial\left(w_{\lambda} \theta_{\lambda}\right)}{\partial Z}\right]=G_{X} \frac{\partial^{2} \theta_{\lambda}}{\partial X^{2}}+G_{Y} \frac{\partial^{2} \theta_{\lambda}}{\partial Y^{2}}+G_{Z} \frac{\partial^{2} \theta_{\lambda}}{\partial Z^{2}}
$$

Utilizando diferenciación en el tiempo artificial para discretizar el término de acumulación se tiene la siguiente expresión:

$$
\frac{1}{\left(\alpha_{\theta}\right)_{\lambda}} \frac{\partial \theta_{\lambda}}{\partial t}=\frac{\theta_{\lambda}^{k+1}-\theta_{\lambda}^{k}}{\left(\alpha_{\theta}\right)_{\lambda} \Delta t}
$$

Definiendo la variable de iteración como la razón del incremento de la temperatura sobre el incremento del tiempo mediante

$$
W_{\theta}=\frac{\theta_{\lambda}^{k+1}-\theta_{\lambda}^{k}}{\Delta t}
$$

O bien en términos de la temperatura adimensional en la iteración $k+1$

$$
\theta_{\lambda}^{k+1}=W_{\theta} \Delta t+\theta_{\lambda}^{k}
$$

Donde el superíndice $k+1$ indica la iteración en el tiempo actual, por lo que la ecuación (3.13) se puede expresar simplemente como:

$$
\theta_{\lambda}=W_{\theta} \Delta t+\theta_{\lambda}^{k}
$$

Sustituyendo (3.11) y (3.12) en (3.10), se obtiene:

$$
\frac{W_{\theta}}{\left(\alpha_{\theta}\right)_{\lambda}}+F\left[\frac{\partial\left(u_{\lambda} \theta_{\lambda}\right)}{\partial X}+\frac{\partial\left(v_{\lambda} \theta_{\lambda}\right)}{\partial Y}+\frac{\partial\left(w_{\lambda} \theta_{\lambda}\right)}{\partial Z}\right]=G_{X} \frac{\partial^{2} \theta_{\lambda}}{\partial X^{2}}+G_{Y} \frac{\partial^{2} \theta_{\lambda}}{\partial Y^{2}}+G_{Z} \frac{\partial^{2} \theta_{\lambda}}{\partial Z^{2}}
$$

Agrupando en términos de la variación en cada una de las direcciones

$$
\frac{W_{\theta}}{\left(\alpha_{\theta}\right)_{\lambda}}+\underbrace{F \frac{\partial\left(u_{\lambda} \theta_{\lambda}\right)}{\partial X}-G_{X} \frac{\partial^{2} \theta_{\lambda}}{\partial X^{2}}}_{\text {Variación en } X}+\underbrace{F \frac{\partial\left(v_{\lambda} \theta_{\lambda}\right)}{\partial Y}-G_{Y} \frac{\partial^{2} \theta_{\lambda}}{\partial Y^{2}}}_{\text {Variación en } Y}+\underbrace{F \frac{\partial\left(w_{\lambda} \theta_{\lambda}\right)}{\partial Z}-G_{Z} \frac{\partial^{2} \theta_{\lambda}}{\partial Z^{2}}}_{\text {Variación en } \mathrm{Z}}=0
$$


Factorizando en $\theta_{\lambda}$

$$
\begin{aligned}
\frac{W_{\theta}}{\left(\alpha_{\theta}\right)_{\lambda}}+ & \theta_{\lambda} \underbrace{\left[F \frac{\partial\left(u_{\lambda}\right)}{\partial X}-G_{X} \frac{\partial^{2}}{\partial X^{2}}\right]}_{\text {Variación en } X} \\
+ & \underbrace{\theta_{\lambda}\left[F \frac{\partial\left(v_{\lambda}\right)}{\partial Y}-G_{Y} \frac{\partial^{2}}{\partial Y^{2}}\right]}_{\text {Variación en } \mathrm{Z}} \\
+ & \underbrace{\theta_{\lambda}\left[F \frac{\partial\left(w_{\lambda}\right)}{\partial Z}-G_{Z} \frac{\partial^{2}}{\partial Z^{2}}\right]}_{\text {Variación en } Y}=0
\end{aligned}
$$

La ecuación anterior se puede simplificar con la inclusión de operadores diferenciales que involucran la diferencia de términos de segundo y primer orden, quedando la ecuación (3.15b) de la siguiente forma:

$$
\frac{W_{\theta}}{\left(\alpha_{\theta}\right)_{\lambda}}=\partial_{X} \theta_{\gamma}+\partial_{Y} \theta_{\gamma}+\partial_{Z} \theta_{\gamma}
$$

Los operadores diferenciales dependientes de cada dirección quedan definidos mediante las siguientes expresiones:

$$
\partial_{X}=\left(G_{X} \frac{\partial^{2}}{\partial X^{2}}-F \frac{\partial u}{\partial X}\right), \quad \partial_{Y}=\left(G_{Y} \frac{\partial^{2}}{\partial Y^{2}}-F \frac{\partial v}{\partial Y}\right), \quad \partial_{Z}=\left(G_{Z} \frac{\partial^{2}}{\partial Z^{2}}-F \frac{\partial w}{\partial Z}\right)
$$

Sustituyendo $\theta$ en términos del valor de la iteración anterior especificado por la ecuación (3.14), factorizando y reordenando la ecuación resultante para la transferencia de energía:

$$
\left[1-\left(\alpha_{\theta}\right)_{\lambda} \Delta t\left(\partial_{X}+\partial_{Y}+\partial_{Z}\right)\right] \frac{W_{\theta}}{\left(\alpha_{\theta}\right)_{\lambda}}=\left(\partial_{X}+\partial_{Y}+\partial_{Z}\right) \theta_{\lambda}^{k} \quad \lambda=\eta, \omega
$$

Para resolver la expresión anterior, se puede dividir en un sistema de tres ecuaciones cuya característica sea la dependencia de cada una de ella con una sola dirección de los ejes coordenados (ADI), de tal manera que con la suma de las tres ecuaciones se tenga como resultado la ecuación original. Para la ecuación de energía este sistema toma la siguiente forma: 


$$
\begin{array}{ll}
{\left[\frac{1}{\left(\alpha_{\theta}\right)_{\lambda}}-\Delta t \partial_{X}\right] W_{\theta}^{\prime \prime}=\left(\partial_{X}+\partial_{Y}+\partial_{Z}\right) \theta_{\lambda}^{k}} & \text { Implícito en } X \\
{\left[\frac{1}{\left(\alpha_{\theta}\right)_{\lambda}}-\Delta t \partial_{Y}\right] W_{\theta}^{\prime}=W_{\theta}^{\prime \prime}} & \text { Implícito en } Y \\
{\left[\frac{1}{\left(\alpha_{\theta}\right)_{\lambda}}-\Delta t \partial_{Z}\right] W_{\theta}=W_{\theta}^{\prime}} &
\end{array}
$$

Para obtener los valores de las variables intermedias $W_{\theta}, W_{\theta}^{\prime}$ y $W_{\theta}^{\prime \prime}$ se resuelven las ecuaciones implícitamente en la dirección correspondiente (ver Figura 3.2); de tal manera que para encontrar el valor de $W_{\theta}$ " en la ecuación (3.19) se resuelve implícitamente en la dirección $X$, la forma total para la ecuación (3.19) es:

$$
\begin{aligned}
\frac{W_{\theta}^{\prime \prime}}{\left(\alpha_{\theta}\right)_{\lambda}}-\Delta t G_{X} \frac{\partial^{2} W_{\theta}^{\prime \prime}}{\partial X^{2}}+\Delta t F \frac{\partial\left(u_{\lambda}^{k} W_{\theta}^{\prime \prime}\right)}{\partial X}= & \underbrace{G_{X}}_{\text {Conducción }} \begin{array}{l}
\underbrace{\frac{\partial^{2} \theta_{\lambda}^{k}}{\partial X^{2}}+G_{Y} \frac{\partial^{2} \theta_{\lambda}^{k}}{\partial Y^{2}}+G_{Z} \frac{\partial^{2} \theta_{\lambda}^{k}}{\partial Z^{2}}}_{\text {Conveción }} \\
-F\left[\frac{\partial\left(u_{\lambda}^{k} \theta_{\lambda}^{k}\right)}{\partial X}+\frac{\partial\left(v_{\lambda}^{k} \theta_{\lambda}^{k}\right)}{\partial Y}+\frac{\partial\left(w_{\lambda}^{k} \theta_{\lambda}^{k}\right)}{\partial Z}\right]
\end{array}
\end{aligned}
$$

Para tener la forma en diferencias finitas de la ecuación anterior se requiere de la forma discretizada de las derivadas utilizando espaciamiento variable, a continuación se presenta la discretización centrada para la primer y segunda derivada, los detalles de la discretización se pueden consultar en el Apéndice D o bien en trabajos previos (Leonardi, 1984 y Valencia, $1993)$.

- Derivada centrada de primer orden

Para ejemplificar la discretización de las derivadas se toma cualquier propiedad $(\xi)$ y su variación con respecto a la coordenada $X$ en la posición $i$, la discretización de la primera derivada se muestra a continuación:

$$
\frac{\partial \xi}{\partial X}=C_{X_{1, j}} \xi_{i-1, j, k}+C_{X_{2, i}} \xi_{i, j, k}+C_{X_{3, i}} \xi_{i+1, j, k}
$$



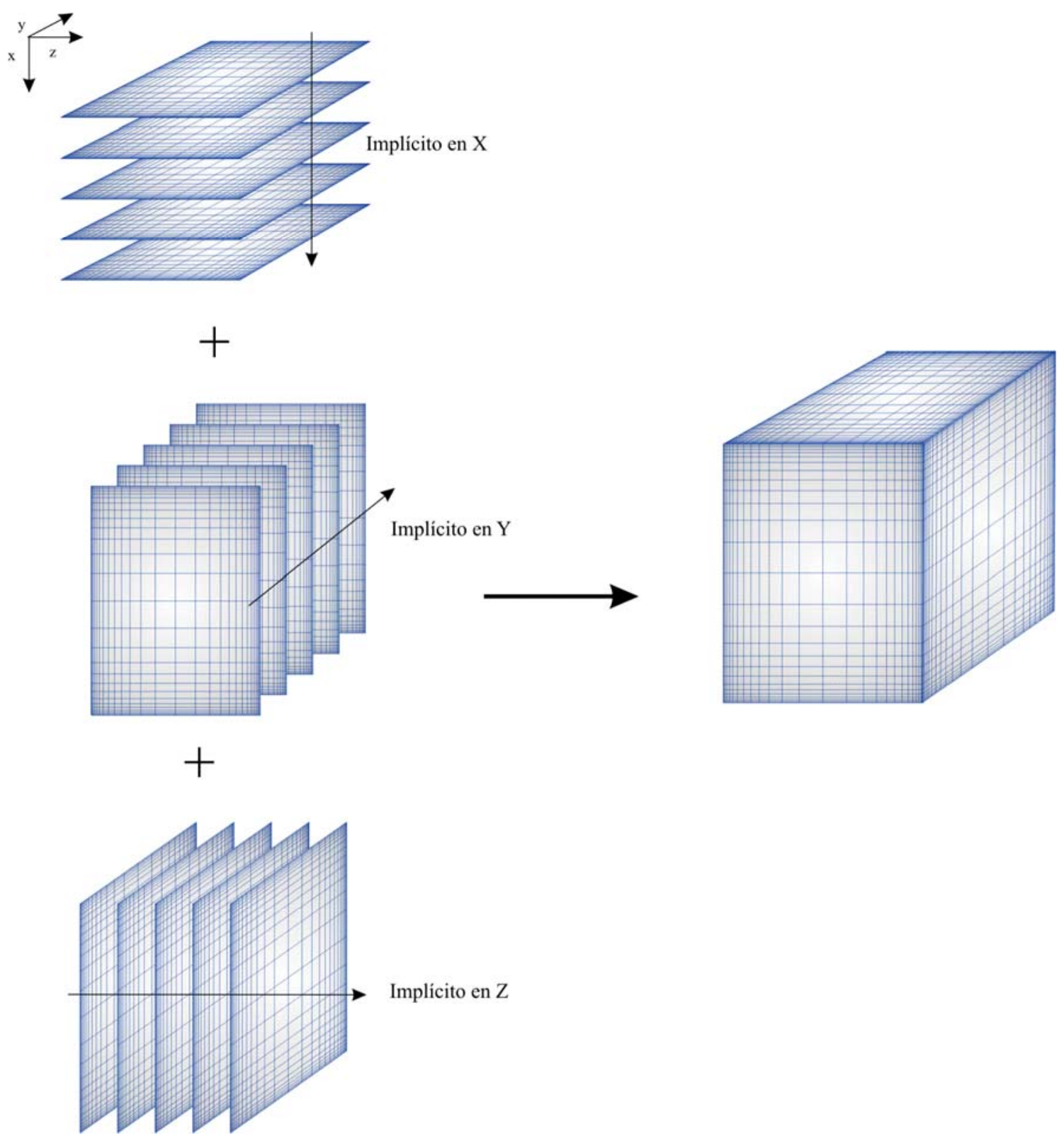

Figura 3.2.- Secuencia de evaluación en el método ADI. 
Los valores de $C_{X_{1, i}}, C_{X_{2, i}}, C_{X_{3, i}}$ involucran los espaciamientos variables que están dados por:

$$
\begin{aligned}
& C_{X_{1, i}}=-\frac{\left(\Delta X_{2}\right)^{2}}{\Delta X_{2}\left(\Delta X_{1}\right)^{2}+\Delta X_{1}\left(\Delta X_{2}\right)^{2}} \\
& C_{X_{2, i}}=\frac{\left(\Delta X_{2}\right)^{2}-\left(\Delta X_{1}\right)^{2}}{\Delta X_{2}\left(\Delta X_{1}\right)^{2}+\Delta X_{1}\left(\Delta X_{2}\right)^{2}} \\
& C_{X_{3, I}}=-\frac{\left(\Delta X_{1}\right)^{2}}{\Delta X_{2}\left(\Delta X_{1}\right)^{2}+\Delta X_{1}\left(\Delta X_{2}\right)^{2}}
\end{aligned}
$$

La ubicación de cada uno de los incrementos para la obtención de las constantes se puede observar en la Figura 3.3. Las derivadas en las direcciones restantes tendrán fórmulas equivalentes.

- Derivada centrada de segundo orden

De forma similar, para la derivada de segundo orden se tiene la siguiente expresión

$$
\frac{\partial^{2} \xi}{\partial X^{2}}=C_{X_{4, i}} \xi_{i-1}+C_{X_{5, i}} \xi_{i}+C_{X_{6, i}} \xi_{i+1}
$$

Donde las constantes de los incrementos variables son:

$$
\begin{aligned}
C_{X_{4, i}} & =\frac{2}{\Delta X_{1}\left(\Delta X_{1}+\Delta X_{2}\right)} \\
C_{X_{5, i}} & =-\frac{2}{\Delta X_{1} \Delta X_{2}} \\
C_{X_{6, i}} & =-\frac{2}{\Delta X_{2}\left(\Delta X_{1}+\Delta X_{2}\right)}
\end{aligned}
$$

Sustituyendo las ecuaciones (3.23)-(3.27) se obtiene como resultado el siguiente sistema tridiagonal para el lecho fluido, en las ecuaciones no se consideran las fronteras ni la interregión, el cálculo para la actualización de estos valores se determinan por separado.

$$
A_{i-1} W_{\theta_{i, j, k}}^{\prime \prime}+B_{i} W_{\theta_{i, j, k}}^{\prime \prime}+C_{i+1} W_{\theta_{i, j, k}}^{\prime \prime}=D_{i} \quad i=2, . ., \mathrm{NXI}-1 ; \mathrm{NXI}+1, \ldots, \mathrm{NX}-1
$$




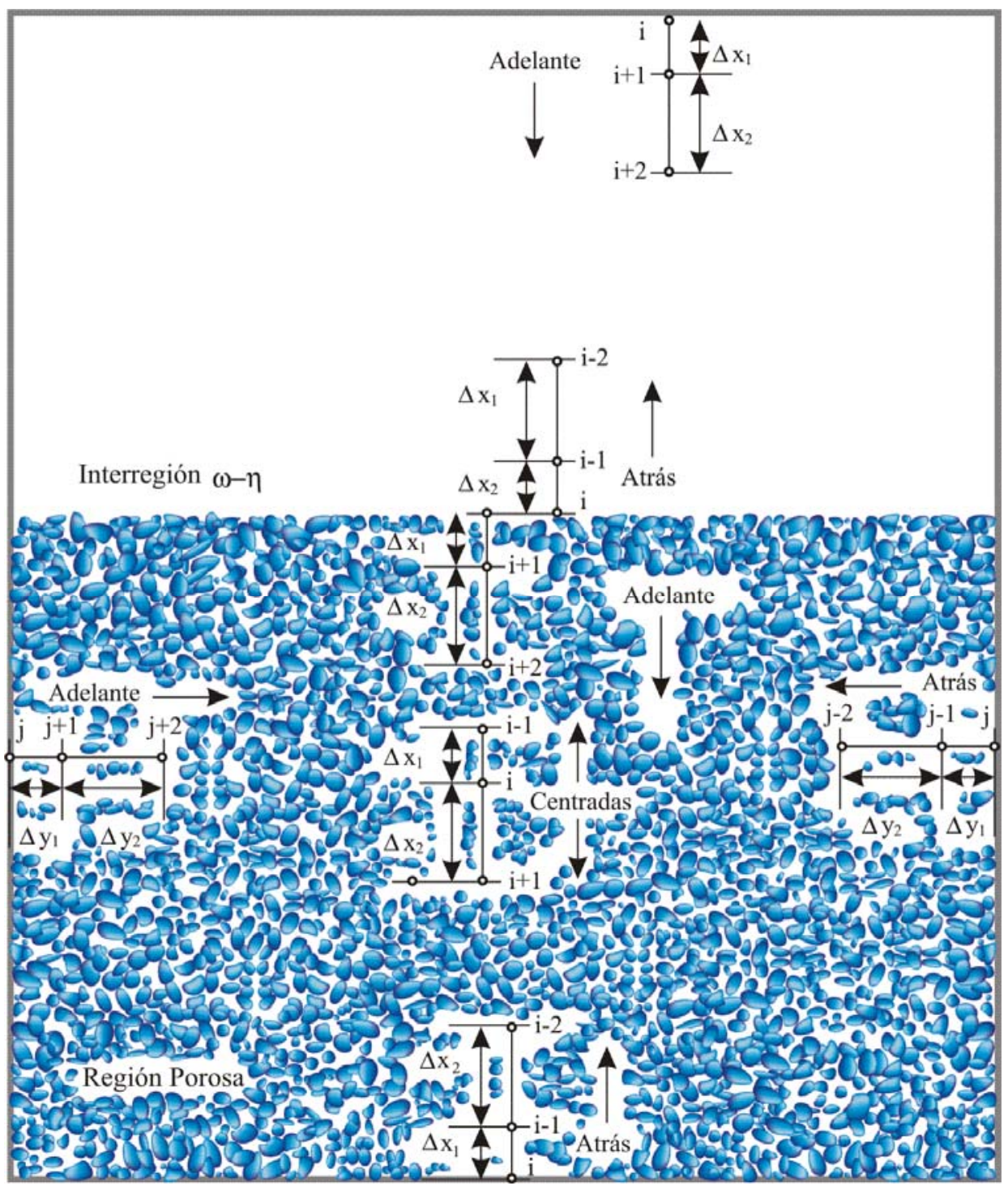

Figura 3.3.- Incrementos variables para la discretización de las ecuaciones. 
Donde:

$$
\begin{aligned}
& A_{i}=-\Delta t\left(G_{X} C_{X_{4, i}}-F C_{X_{1, i}} u_{i-1, j, k}\right) \\
& B_{i}=\frac{1}{\left(\alpha_{\theta}\right)_{\lambda}}-\Delta t\left(G_{X} C_{X_{5, i}}-F C_{X_{2, i}} u_{i, j, k}\right) \\
& C_{i}=-\Delta t\left(G_{X} C_{X_{6, i}}-F C_{X_{3, i}} u_{i+1, j, k}\right) \\
& D_{i}=\underbrace{G_{X}\left(C_{X_{4, i}} \theta_{i-1, j, k}^{k}+C_{X_{5, j}} \theta_{i, j, k}^{k}+C_{X_{6, i}} \theta_{i+1, j, k}^{k}\right)}_{G_{X} \frac{\partial^{2} \theta_{\lambda}^{k}}{\partial X^{2}}} \\
& -\underbrace{F\left(C_{X_{1, i}} u_{i-1, j, k}^{k} \theta_{i-1, j, k}^{k}+C_{X_{2, i}}^{k} u_{i, j, k}^{k} \theta_{i, j, k}^{k}+C_{X_{3, i}} u_{i+1, j, k}^{k} \theta_{i+1, j, k}^{k}\right)}_{F \frac{\partial\left(u_{\lambda}^{k} \theta_{\lambda}^{k}\right)}{\partial X}} \\
& +\underbrace{G_{Y}\left(C_{Y_{4, j}} \theta_{i, j-1, k}^{k}+C_{Y_{5, j}} \theta_{i, j, k}^{k}+C_{Y_{6, j}} \theta_{i, j+1, k}^{k}\right)}_{G_{Y} \frac{\partial^{2} \theta_{\lambda}^{k}}{\partial Y^{2}}} \\
& -\underbrace{F\left(C_{Y_{1, j}} v_{i, j-1, k}^{k} \theta_{i, j-1, k}^{k}+C_{Y_{2, j}} v_{i, j, k}^{k} \theta_{i, j, k}^{k}+C_{Y_{3, j}} v_{i, j+1, k}^{k} \theta_{i, j+1, k}^{k}\right)}_{F \frac{\partial\left(v_{\lambda}^{k} \theta_{\lambda}^{k}\right)}{\partial Y}} \\
& +\underbrace{G_{Z}\left(C_{Z_{4, k}} \theta_{i, j, k-1}^{k}+C_{Z_{5, k}} \theta_{i, j, k}^{k}+C_{Z_{6, k}} \theta_{i, j, k+1}^{k}\right)}_{G_{Z} \frac{\partial^{2} \theta_{\lambda}^{k}}{\partial Z^{2}}} \\
& \underbrace{-F\left(C_{Z_{1, k}} w_{i, j, k-1}^{k} \theta_{i, j, k-1}^{k}+C_{Z_{2, k}} w_{i, j, k}^{k} \theta_{i, j, k}^{k}+C_{Z_{3, k}} w_{i, j, k+1}^{k} \theta_{i, j, k+1}^{k}\right)}_{F \frac{\partial\left(w_{\lambda}^{k} \theta_{\lambda}^{k}\right)}{\partial Z}}
\end{aligned}
$$

La expansión de la ecuación (3.31) forma una matriz tridiagonal:

$$
\left[\begin{array}{ccccccccc}
B_{2} & C_{2} & 0 & 0 & \cdots & 0 & 0 & 0 & 0 \\
A_{3} & B_{3} & C_{3} & 0 & \cdots & 0 & 0 & 0 & 0 \\
0 & A_{4} & B_{4} & C_{4} & \cdots & 0 & 0 & 0 & 0 \\
\vdots & \vdots & \vdots & \vdots & \ddots & \vdots & \vdots & \vdots & \vdots \\
0 & 0 & 0 & 0 & \cdots & A_{N X I-3} & B_{N X I-3} & C_{N X I-3} & 0 \\
0 & 0 & 0 & 0 & \cdots & 0 & A_{N X I-2} & B_{N X I-2} & C_{N X I-2} \\
0 & 0 & 0 & 0 & \cdots & 0 & 0 & A_{N X I-1} & B_{N X I-1}
\end{array}\right]\left[\begin{array}{c}
W_{2}^{\prime \prime} \\
W_{3}^{\prime \prime} \\
W_{4}^{\prime \prime} \\
\vdots \\
W_{N X I-3}^{\prime \prime} \\
W_{N X I-2}^{\prime \prime} \\
W_{N X I-1}^{\prime \prime}
\end{array}\right]=\left[\begin{array}{c}
D_{2}-A_{2} W_{1}^{\prime \prime} \\
D_{3} \\
D_{4} \\
\vdots \\
D_{N X I-3} \\
D_{N X I-2} \\
D_{N X I-1}-C_{N X I-1} W_{8}^{\prime \prime}
\end{array}\right]
$$


El sistema tridiagonal se resuelve utilizando el algoritmo de Thomas (Ames, 1992; Dagtekin y Oztop, 2001; Provitsky y Morris, 2000) para obtener el valor de $W^{\prime \prime}$, con este se puede calcular $W^{\prime}$ (implícito en $Y$ ) aplicando el mismo procedimiento de discretización y solución tridiagonal anterior, ver (Figura 3.2), el sistema en esta dirección está dado por el siguiente conjunto de ecuaciones:

$$
A_{j} W_{\theta_{i, j-1, k}}^{\prime}+B_{j} W_{\theta_{i, j, k}}^{\prime}+C_{j} W_{\theta_{i, j+1, k}}^{\prime}=D_{j} \quad j=2, . ., \mathrm{NY}-1
$$

Con

$$
\begin{aligned}
& A_{j}=-\Delta t\left(G_{Y} C_{Y_{4, j}}-F C_{Y_{1, j}} u_{i, j-1, k}\right) \\
& B_{j}=1-\Delta t\left(G_{Y} C_{Y_{5, j}}-F C_{Y_{2, j}} u_{i, j, k}\right) \\
& C_{j}=-\Delta t\left(G_{Y} C_{Y_{6, j}}-F C_{Y_{3, j}} u_{i, j+1, k}\right) \\
& D_{j}=W^{\prime \prime}
\end{aligned}
$$

Finalmente con el valor de $W^{\prime}$ se calcula $W$, que es la variable que involucra la temperatura adimensional en la iteración actual, el sistema resultante de la discretización en diferencias finitas es el siguiente:

$$
A_{k} W_{\theta_{i, j, k-1}}+B_{k} W_{\theta_{i, j, k}}+C_{k} W_{\theta_{i, j, k+1}}=D_{k} \quad k=2, . ., \mathrm{NZ}-1
$$

Con

$$
\begin{aligned}
& A_{k}=-\Delta t\left(G_{Z} C_{Z_{4, k}}-F C_{Z_{1, k}} u_{i, j, k-1}\right) \\
& B_{k}=1-\Delta t\left(G_{Z} C_{Z_{5, j}}-F C_{Z_{2, k}} u_{i, j, k}\right) \\
& C_{k}=-\Delta t\left(G_{Z} C_{Z_{6, k}}-F C_{Z_{3, k}} u_{i, j, k+1}\right) \\
& D_{k}=W^{\prime}
\end{aligned}
$$

Con el vector $W$ se evalúa la ecuación (3.14) para obtener los nuevos valores de la temperatura adimensional. El proceso iterativo se aplica en forma semejante para cada una de las direcciones de la región porosa.

\section{Vector potencial}

A diferencia de la variable temperatura, para el caso de una variable vectorial se tiene que desarrollar el método ADI para cada una de las componentes del vector. Para ejemplificar lo anterior se mostrará el procedimiento para la componente " $n$ " del vector potencial, expresando las ecuaciones (3.1) y (3.5) mediante:

$$
\frac{1}{\left(\alpha_{\Psi}\right)_{\eta}} \frac{\partial \Psi_{v, \gamma}}{\partial t}=\frac{\partial^{2} \Psi_{n, \gamma}}{\partial X^{2}}+\frac{\partial^{2} \Psi_{n, \gamma}}{\partial Y^{2}}+\frac{\partial^{2} \Psi_{n, \gamma}}{\partial Z^{2}}-\zeta_{n, \gamma} \quad n=X, Y, Z ; \quad \gamma=\eta, \omega
$$


Realizando un procedimiento similar al presentado para la ecuación de energía, se tiene la ecuación para el vector potencial en términos de la variable $W_{\Psi}$ :

$$
\Psi_{n, \gamma}=W_{\Psi} \Delta t+\Psi_{n, \gamma}^{k}
$$

Sustituyendo (3.48) en (3.47) se obtiene:

$$
\frac{\Psi_{v, \gamma}^{k+1}-\Psi_{v, \gamma}^{k}}{\left(\alpha_{\Psi}\right)_{\eta} \Delta t}=\frac{\partial^{2} \Psi_{n, \gamma}}{\partial X^{2}}+\frac{\partial^{2} \Psi_{n, \gamma}}{\partial Y^{2}}+\frac{\partial^{2} \Psi_{n, \gamma}}{\partial Z^{2}}-\zeta_{n, \gamma}
$$

o bien en términos de operadores diferenciales de segundo orden

$$
\frac{W_{\Psi}}{\left(\alpha_{\Psi}\right)_{\eta}}=\Delta t\left(\partial_{X}^{2}+\partial_{Y}^{2}+\partial_{Z}^{2}\right) \Psi_{n, \gamma}-\zeta_{n, \gamma}
$$

En la ecuación anterior, los operadores diferenciales quedan definidos por términos de segundo orden, a diferencia de la ecuación de temperatura en la cual se incluía una diferencia de términos de primer y segundo orden:

$$
\partial_{X}^{2}=\frac{\partial^{2}}{\partial X^{2}} \quad \partial_{Y}^{2}=\frac{\partial^{2}}{\partial Y^{2}} \quad \partial_{Z}^{2}=\frac{\partial^{2}}{\partial Z^{2}}
$$

Ahora, sustituyendo (3.48) en (3.50) se obtiene:

$$
\frac{W_{\Psi}}{\left(\alpha_{\Psi}\right)_{\eta}}=\Delta t\left(\partial_{X}^{2}+\partial_{Y}^{2}+\partial_{Z}^{2}\right) W_{\Psi}+\left(\partial_{X}^{2}+\partial_{Y}^{2}+\partial_{Z}^{2}\right) \Psi_{n, \gamma}^{k}-\zeta_{n, \gamma}
$$

Factorizando y reordenando la ecuación anterior, se tiene:

$$
\left[1-\left(\alpha_{\Psi}\right)_{\eta} \Delta t\left(\partial_{X}^{2}+\partial_{Y}^{2}+\partial_{Z}^{2}\right)\right] \frac{W_{\Psi}}{\left(\alpha_{\Psi}\right)_{\eta}}=\left(\partial_{X}^{2}+\partial_{Y}^{2}+\partial_{Z}^{2}\right) \Psi_{n, \gamma}^{k}-\zeta_{n, \gamma}
$$

La descomposición para la integración implícita en cada una de las direcciones es la siguiente:

$$
\left[\frac{1}{\left(\alpha_{\Psi}\right)_{\eta}}-\Delta t \partial_{X}^{2}\right] W_{\Psi}^{\prime}=\left(\partial_{X}^{2}+\partial_{Y}^{2}+\partial_{Z}^{2}\right) \Psi_{n, \gamma}^{k}-\zeta_{n, \gamma} \quad \text { Implícito en } X
$$




$$
\begin{array}{ll}
{\left[\frac{1}{\left(\alpha_{\Psi}\right)_{\eta}}-\Delta t \partial_{Y}^{2}\right] W_{\Psi}^{\prime}=W_{\Psi}^{\prime \prime}} & \text { Implícito en } Y \\
{\left[\frac{1}{\left(\alpha_{\Psi}\right)_{\eta}}-\Delta t \partial_{Z}^{2}\right] W_{\Psi}=W_{\Psi}^{\prime}} & \text { Implícito en } Z
\end{array}
$$

Como cada una de las ecuaciones (3.54), (3.55) y (3.56) dependen de una dirección, se pueden resolver implícitamente en la dirección del eje correspondiente (Samarskii y Andreyev, 1963), haciendo notar que cuando se alcanza el estado estacionario (artificial) la suma de las tres ecuaciones tiene como resultado la ecuación (3.47). La discretización y solución de la ecuación (3.48) (implícito en $X$ ) es:

$$
\frac{W_{\Psi_{X}}^{\prime \prime}}{\left(\alpha_{\Psi}\right)_{\eta}}-\Delta t \frac{\partial^{2} W_{\Psi_{X}}^{\prime \prime}}{\partial X^{2}}=\left(\frac{\partial^{2} \Psi_{n, \gamma}}{\partial X^{2}}+\frac{\partial^{2} \Psi_{n, \gamma}}{\partial Y^{2}}+\frac{\partial^{2} \Psi_{n, \gamma}}{\partial Z^{2}}\right)-\zeta_{n, \gamma}
$$

La discretización de las derivadas espaciales se realiza en diferencias centradas utilizando las ecuaciones (3.23)-(3.26) o sus formas similares con sus correspondientes constantes, obteniéndose la expresión:

$$
A_{i} W_{\Psi_{i-1, j k}}^{\prime \prime}+B_{i} W_{\Psi_{i, j, k}}^{\prime \prime}+C_{i} W_{\Psi_{i+1, j k}}^{\prime \prime}=D_{i} \quad i=2, \ldots, N X I-1
$$

Con las siguientes expresiones para las constantes $A, B, C$ y $D$

$$
\begin{aligned}
& A_{i}=-\left(\alpha_{\Psi}\right)_{\eta} \Delta t C_{X_{4, i}} \\
& B_{i}=-\left(\alpha_{\Psi}\right)_{\eta} \Delta t C_{X_{5, i}}-1 \\
& C_{i}=-\left(\alpha_{\Psi}\right)_{\eta} \Delta t C_{X_{6, i}} \\
& D_{i}=\zeta_{X_{i, j, k}}+\Psi_{X_{i, j, k}}\left(C_{X_{5, i}}+C_{Y_{5, j}}+C_{Z_{5, k}}\right)+\Psi_{X_{i-1, j, k}}\left(C_{X_{4, i}}\right)+\Psi_{X_{i+1, j, k}}\left(C_{X_{6, i}}\right) \\
& +\Psi_{X_{i, j, 1, k}}\left(C_{Y_{4, j}}\right)+\Psi_{X_{i, j, j+1, k}}\left(C_{Y_{6, j}}\right)+\Psi_{X_{i, j, k-1}}\left(C_{Z_{4, k}}\right)+\Psi_{X_{i, j, k+1}}\left(C_{Z_{6, k}}\right)
\end{aligned}
$$

El sistema tridiagonal adquiere la forma presentada en la ecuación (3.36), el cual se resuelve por el algoritmo de Thomas (Dagtekin y Oztop, 2001; Provitsky y Morris, 2000; Lapidus y Pinder, 1982), posteriormente se resuelven las ecuaciones (3.55) y (3.56) y finalmente se aplica la ecuación (3.48) para obtener el valor de la componente del vector potencial. 


\subsubsection{Ecuación de transferencia de masa}

La similitud que tienen las ecuaciones de energía y masa simplifica la aplicación de la discretización y del método de integración (ADI).

Cualquiera de las ecuaciones de transferencia de masa ecuaciones (3.4) o (3.8) se puede representar mediante:

$$
\frac{1}{\left(\alpha_{\phi}\right)_{\omega}} \frac{\partial \phi_{\eta}}{\partial t}+H \nabla \cdot\left(\mathbf{v}_{\gamma} \phi_{\gamma}\right)=J_{X} \frac{\partial^{2} \phi_{\gamma}}{\partial X^{2}}+J_{Y} \frac{\partial^{2} \phi_{\gamma}}{\partial Y^{2}}+J_{Z} \frac{\partial^{2} \phi_{\gamma}}{\partial Z^{2}}
$$

Siguiendo el mismo procedimiento presentado para la ecuación de transporte de energía, primero se resuelve para la dirección X:

$$
\begin{aligned}
& \frac{W_{\phi}^{\prime \prime}}{\left(\alpha_{\phi}\right)_{\lambda}}-\Delta t J_{X} \frac{\partial^{2} W_{\phi}^{\prime \prime}}{\partial X^{2}}+\Delta t H \frac{\partial\left(u_{\lambda}^{k} W_{\phi}^{\prime \prime}\right)}{\partial X}=\underbrace{J_{X} \frac{\partial^{2} \phi_{\lambda}^{k}}{\partial X^{2}}+J_{Y} \frac{\partial^{2} \phi_{\lambda}^{k}}{\partial Y^{2}}+J_{Z} \frac{\partial^{2} \phi_{\lambda}^{k}}{\partial Z^{2}}}_{\text {Difusión }} \\
& \underbrace{-H\left[\frac{\partial\left(u_{\lambda}^{k} \phi_{\lambda}^{k}\right)}{\partial X}+\frac{\partial\left(v_{\lambda}^{k} \phi_{\lambda}^{k}\right)}{\partial Y}+\frac{\partial\left(w_{\lambda}^{k} \phi_{\lambda}^{k}\right)}{\partial Z}\right]}_{\text {Conveción }}
\end{aligned}
$$

Después de aplicar las ecuaciones (3.9)-(3.16) se obtiene el siguiente sistema tridiagonal

$$
A_{i} W_{\phi_{i-1, j, k}}^{\prime \prime}+B_{i} W_{\phi_{i, j, k}}^{\prime \prime}+C_{i} W_{\phi_{i+1, j, k}}^{\prime \prime}=D_{i}
$$

Donde:

$$
\begin{aligned}
& A_{i}=-\Delta t\left(J_{X} C_{X_{4, i}}-H C_{X_{1, i}} u_{i-1, j, k}\right) \\
& B_{i}=\frac{1}{\left(\alpha_{\phi}\right)_{\lambda}}-\Delta t\left(J_{X} C_{X_{5, i}}-H C_{X_{2, i}} u_{i, j, k}\right) \\
& C_{i}=-\Delta t\left(J_{X} C_{X_{6, i}}-H C_{X_{3, i}} u_{i+1, j, k}\right) \\
& D_{i}=\underbrace{J_{X}\left(C_{X_{4, i}} \phi_{i-1, j, k}^{k}+C_{X_{5, i}} \phi_{i, j, k}^{k}+C_{X_{6, i}} \phi_{i+1, j, k}^{k}\right)}_{J_{X} \frac{\partial^{2} \phi_{\lambda}^{k}}{\partial X^{2}}}
\end{aligned}
$$




$$
\begin{aligned}
& -\underbrace{H\left(C_{X_{1, i}} u_{i-1, j, k}^{k} \phi_{i-1, j, k}^{k}+C_{X_{2, i}}^{k} u_{i, j, k}^{k} \phi_{i, j, k}^{k}+C_{X_{3, i}} u_{i+1, j, k}^{k} \phi_{i+1, j, k}^{k}\right)}_{H \frac{\partial\left(u_{\lambda}^{k} \phi_{\lambda}^{k}\right)}{\partial X}} \\
& +\underbrace{J_{Y}\left(C_{Y_{4, j}} \phi_{i, j-1, k}^{k}+C_{Y_{5, j}} \phi_{i, j, k}^{k}+C_{Y_{6, j}} \phi_{i, j+1, k}^{k}\right)}_{J_{Y} \frac{\partial^{2} \phi_{\lambda}^{k}}{\partial Y^{2}}} \\
& \underbrace{-H\left(C_{Y_{1, j}} v_{i, j-1, k}^{k} \phi_{i, j-1, k}^{k}+C_{Y_{2, j}} v_{i, j, k}^{k} \phi_{i, j, k}^{k}+C_{Y_{3, j}} v_{i, j+1, k}^{k} \phi_{i, j+1, k}^{k}\right)}_{H \frac{\partial\left(v_{\lambda}^{k} \phi_{\lambda}^{k}\right)}{\partial Y}} \\
& +\underbrace{J_{Z}\left(C_{Z_{4, k}} \phi_{i, j, k-1}^{k}+C_{Z_{5, k}} \phi_{i, j, k}^{k}+C_{Z_{6, k}} \phi_{i, j, k+1}^{k}\right)}_{J_{Z} \frac{\partial^{2} \phi_{\lambda}^{k}}{\partial Z^{2}}} \\
& \underbrace{-H\left(C_{Z_{1, k}} w_{i, j, k-1}^{k} \phi_{i, j, k-1}^{k}+C_{Z_{2, k}} w_{i, j, k}^{k} \phi_{i, j, k}^{k}+C_{Z_{3, k}} w_{i, j, k+1}^{k} \phi_{i, j, k+1}^{k}\right)}_{H \frac{\partial\left(w_{\lambda}^{k} \phi_{\lambda}^{k}\right)}{\partial Z}}
\end{aligned}
$$

\section{Ecuación vector vorticidad}

Para la discretización del vector vorticidad se realiza un procedimiento similar al utilizado por el vector potencial, de tal manera que para la componente $\lambda$ se tiene:

$$
\begin{aligned}
\frac{1}{\left(\alpha_{\zeta}\right)_{\eta}} \frac{\partial W^{\prime \prime}}{\partial t}-\Delta t \operatorname{Pr} \frac{\partial W^{\prime \prime}}{\partial X}= & -\nabla \times\left(\zeta_{\lambda}^{k} \times \mathbf{v}_{\lambda}^{k}\right)+\operatorname{Pr} \nabla^{2} \zeta_{\lambda}^{k} \\
& -\left(R a_{\theta} \operatorname{Pr}\right) \nabla \times\left(\mathbf{i} \theta_{\lambda}^{k}\right)-\left(\frac{R a_{\phi} \operatorname{Pr}}{L e}\right) \nabla \times\left(\mathbf{i} \phi_{\lambda}^{k}\right)
\end{aligned}
$$

Con la adimensionalización del vector vorticidad se tiene el siguiente sistema de ecuaciones:

$$
\begin{aligned}
& A_{i} W_{\zeta_{i-1, j, k}}^{\prime \prime}+B_{i} W_{\zeta_{i, j, k}}^{\prime \prime}+C_{i} W_{\zeta_{i+1, j, k}}^{\prime \prime}=D_{i} \\
& A_{i}=-\Delta \operatorname{tPr} C_{X_{4, i}} \\
& B_{i}=-\Delta \operatorname{tPr} C_{X_{5, i}}-\frac{1}{\left(\alpha_{\zeta}\right)_{\lambda}} \\
& C_{i}=-\Delta \operatorname{Pr} C_{X_{6, i}} \\
& D_{i}=-\underbrace{\zeta_{X_{i, j-1, k}} C_{Y_{1, j}} V_{i, j-1, k}-\zeta_{X_{i, j, k}} C_{Y_{2, j}} V_{i, j, k}-\zeta_{X_{i, j+1, k}} C_{Y_{3, j}} V_{i, j+1, k}}_{\frac{d\left(\zeta_{X} v\right)}{d Y}}
\end{aligned}
$$




$$
\begin{aligned}
& +\underbrace{\zeta_{Y_{i, j-1, k}} C_{Y_{1, j}} u_{i, j-1, k}+\zeta_{Y_{i, j, k}} C_{Y_{2, j}} u_{i, j, k}+\zeta_{X_{i, j+1, k}} C_{Y_{3, j}} u_{i, j+1, k}}_{\frac{d\left(\zeta_{Y} u\right)}{d Y}} \\
& -\underbrace{\zeta_{X_{i, j, k-1}} C_{Z_{1, k}} w_{i, j, k-1}-\zeta_{Y_{i, j, k}} C_{Z_{2, k}} w_{i, j, k}-\zeta_{X_{i, j, k+1}} C_{Z_{3, k}} w_{i, j, k+1}}_{\frac{d\left(\zeta_{X} w\right)}{d Z}} \\
& +\underbrace{\zeta_{Z_{i, j, k-1}} C_{Z_{1, k}} u_{i, j, k-1}+\zeta_{Z_{i, j, k}} C_{Z_{2, k}} u_{i, j, k}+\zeta_{Z_{i, j, k+1}} C_{Z_{3, k}} u_{i, j, k+1}}_{\frac{d\left(\zeta_{X} w\right)}{d Z}} \\
& \underbrace{\operatorname{Pr}\left(\zeta_{X_{i-1, j, k}} C_{X_{4, i}}+\zeta_{X_{i, j, k}} C_{X_{5, i}}+\zeta_{X_{i+1, j, k}} C_{X_{6, i}}\right)}_{\operatorname{Pr} \frac{d^{2}\left(\zeta_{X}\right)}{d X^{2}}} \\
& +\underbrace{\operatorname{Pr}\left(\zeta_{X_{i, j-1, k}} C_{Y_{4, j}}+\zeta_{X_{i, j, k}} C_{Y_{5, j}}+\zeta_{X_{i, j+1, k}} C_{Y_{6, i}}\right)}_{\operatorname{Pr} \frac{d^{2}\left(\zeta_{X}\right)}{d Y^{2}}} \\
& \underbrace{\operatorname{Pr}\left(\zeta_{X_{i, j, k-1}} C_{Z_{4, k}}+\zeta_{X_{i, j, k}} C_{Z_{5, k}}+\zeta_{X_{i, j, k+1}} C_{Z_{6, k}}\right)}_{\operatorname{Pr} \frac{d^{2}\left(\zeta_{X}\right)}{d Z^{2}}} \\
& \underbrace{\left(R a_{\theta} P r\right)\left(\theta_{i, j-1, k} C_{Z_{1, j}}-\theta_{i, j, k} C_{Z_{2, j}}-\theta_{i, j+1, k} C_{Z_{3, j}}\right)}_{\left(R a_{\theta} P r\right) \frac{d^{2}\left(\theta_{Y}\right)}{d Z^{2}}} \\
& +\underbrace{\left(R a_{\theta} \operatorname{Pr}\right)\left(\theta_{i, j, k-1} C_{Y_{1, j}}-\theta_{i, j, k} C_{Y_{2, j}}-\theta_{i, j, k+1} C_{Y_{3, j}}\right)}_{\left(R a_{\theta} P r\right) \frac{d^{2}\left(\theta_{Z}\right)}{d Y^{2}}} \\
& \underbrace{-\left(\frac{R a_{\phi} P r}{L e}\right)\left(\phi_{i, j-1, k} C_{Z_{1, j}}-\phi_{i, j, k} C_{Z_{2, j}}-\phi_{i, j+1, k} C_{Z_{3, j}}\right)}_{\left(\frac{R a_{\phi} P r}{L e}\right) \frac{d^{2}\left(\phi_{Y}\right)}{d Z^{2}}} \\
& \underbrace{\left(\frac{R a_{\phi} P r}{L e}\right)\left(\phi_{i, j, k-1} C_{Y_{1, j}}-\phi_{i, j, k} C_{Y_{2, j}}-\phi_{i, j, k+1} C_{Y_{3, j}}\right)}_{\left(\frac{R a_{\phi} P r}{L e}\right) \frac{d^{2}\left(\phi_{2}\right)}{d Y^{2}}}
\end{aligned}
$$

El procedimiento de integración para las diferentes direcciones se realiza como en las ecuaciones anteriores. 


\subsection{Condiciones de frontera e interregionales.}

Las condiciones de frontera e interregionales (ver Figura 3.4) se establecen para las variables temperatura, masa, vector potencial y vector vorticidad, para estas últimas las condiciones de frontera quedan determinadas por las condiciones de no-deslizamiento y no penetración del vector velocidad planteadas en el Capítulo 2. En la presente sección se plantean las condiciones de frontera e interregionales en términos de diferencias finitas.

\section{- Vector potencial}

Para el vector potencial se debe de cumplir la condición de no penetración en las paredes, expresada mediante:

$$
\frac{\partial\left(\Psi_{i}\right)_{n}}{\partial n}=0 \quad i=\gamma, \beta
$$

Para ser congruentes con el desarrollo que se realizó para el potencial, se ejemplificará el planteamiento de las condiciones frontera para $\Psi_{X, \beta}$. El desarrollo es idéntico al presentado para obtener las ecuaciones generales para el vector vorticidad obteniéndose una matriz tridiagonal cambiando el término independiente $D_{i}$.

Para $j=1$

$$
\begin{aligned}
D_{i}= & \zeta_{X_{i, 1, k}}+\Psi_{X_{i, 1, k}}\left(C_{X_{5 i}}+C_{Y_{5 j}}+C_{Z_{5 k}}\right)+\Psi_{X_{i-1,1, k}} C_{X_{4 i}}+\Psi_{X_{i+1, j, k}} C_{X_{6 i}} \\
& +\Psi_{X_{i, 0, k}} C_{Y_{4 i}}+\Psi_{X_{i, 2, k}} C_{Y_{6 j}}+\Psi_{X_{i, j, k-1}} C_{Z_{4 k}}+\Psi_{X_{i, j, k+1}} C_{Z_{6 i}}
\end{aligned}
$$

De la ecuación (3.77), utilizando discretización hacia adelante se obtiene: $\Psi_{i, 1, k}=\Psi_{1,0, k}$. Sustituyendo esta expresión se obtiene:

$$
\begin{aligned}
D_{i}= & \zeta_{X_{i, 1, k}}+\Psi_{X_{i, 1, k}}\left(C_{X_{5 i}}+C_{Y_{5 L}}+C_{Z_{5 k}}\right)+\Psi_{X_{i-1,1, k}} C_{X_{4 i}}+\Psi_{X_{X+1,1, k}} C_{X_{6 i}} \\
& +2 \Psi_{X_{i, 2, k}} C_{Y_{6 L}}+\Psi_{X_{i, j, k-1}} C_{Z_{4 k}}+\Psi_{X_{i, j, k+1}} C_{Z_{6 k}}
\end{aligned}
$$

Donde:

$$
\begin{aligned}
C_{Y_{6 L}} & =\frac{C_{Y_{6 j}}}{2} \\
C_{Y_{5 L}} & =C_{Y_{5 L}}+C_{Y_{5 j}}
\end{aligned}
$$

Este mismo desarrollo se realiza para $j=N Y$, en las direcciones restantes y para cada una de las regiones. 

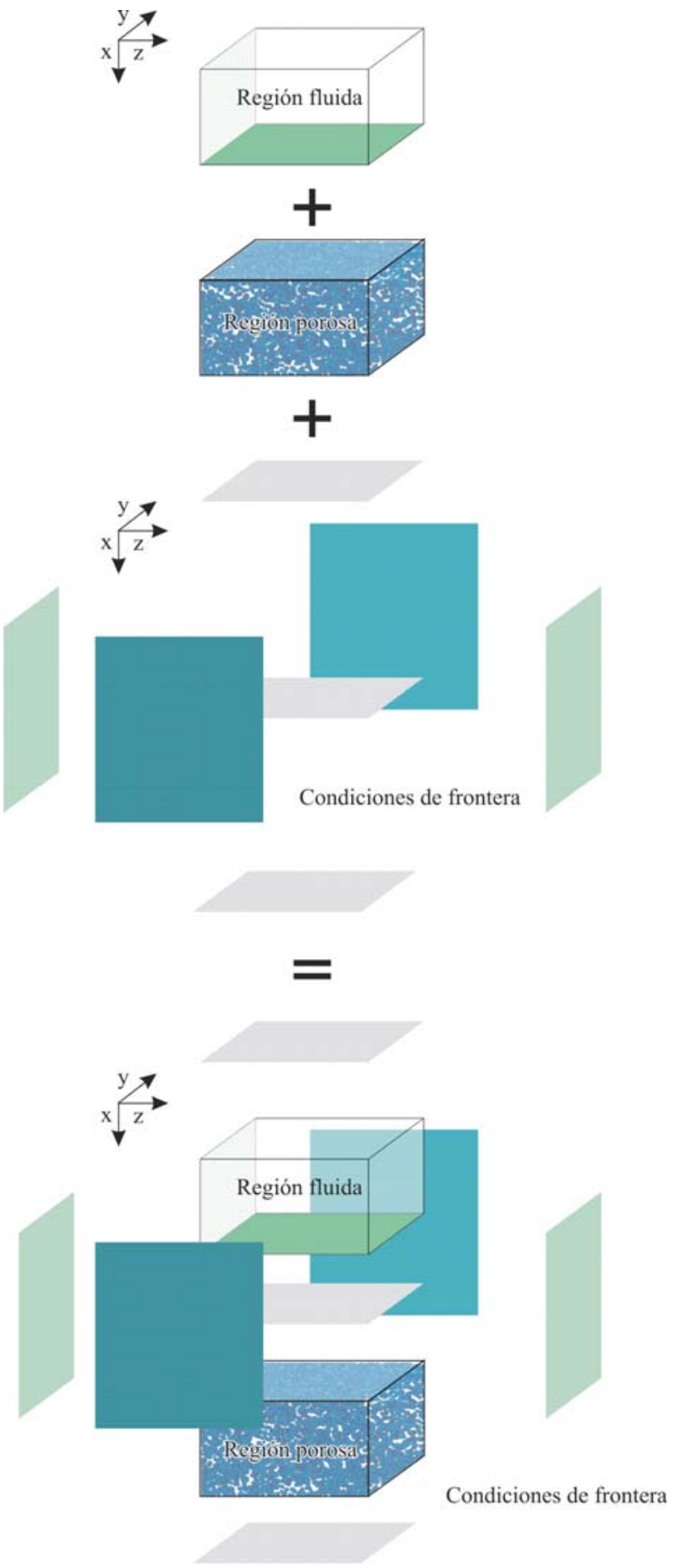

Figura 3.4.- Secuencia para la evaluación de las condiciones frontera e interregional. 
- Condiciones de frontera para la ecuación de energía

La forma discretizada de las condiciones frontera (2.9a)-(2.9c) son:

Dirichlet

$$
\theta_{\lambda, n=\kappa n}=a \quad 0 \leq a \leq 1, \quad \lambda=\omega, \eta, \quad \kappa n=1, X N, Y N, Z N
$$

Newman:

Cauchy:

$$
\left.\frac{\partial \theta_{\lambda}}{\partial n}\right|_{n=\kappa n}=a \quad 0 \leq a \leq 1, \quad \lambda=\omega, \eta, \quad \kappa n=1, X N, Y N, Z N
$$

$$
\left.\frac{\partial \theta_{\lambda}}{\partial n}\right|_{n=\kappa n}=b \theta_{\lambda, n=\kappa n}+c \quad 0 \leq b, c \leq 1, \lambda=\omega, \eta, \quad \kappa n=1, X N, Y N, Z N
$$

La forma adimensional para las condiciones tipo Dirichlet, ecuación (3.81) está dada por:

$$
\begin{array}{ll}
\theta_{1}=\theta_{2} & \text { Discretización hacia adelante } \\
\theta_{L}=\theta_{L-1} & \text { Discretización hacia atrás }
\end{array}
$$

Newman

En las condiciones tipo Newman se aplican diferencias finitas hacia adelante o hacia atrás dependiendo de la posición en que se encuentre como se muestra en la Figura 3.3. Para las fronteras ubicadas en $\kappa n=1$, se utilizan diferencias hacia delante

Discretización hacia delante:

$$
\left.\frac{\partial \theta_{\lambda}}{\partial n}\right|_{i=1}=C F_{n_{1}} \theta_{i}+C F_{n_{2}} \theta_{i+1}+C F_{n_{3}} \theta_{i+2}=a
$$

Donde:

$$
\begin{aligned}
& C F_{n_{1}}=-\frac{\Delta n_{i}+2 \Delta n_{i+1}}{\Delta n_{i+1}\left(\Delta n_{i}+\Delta n_{i+1}\right)} \\
& C F_{n_{2}}=\frac{\Delta n_{i}+\Delta n_{i+1}}{\Delta n_{i} \Delta n_{i+1}} \\
& C F_{n_{3}}=-\frac{\Delta n_{i+1}}{\Delta n_{i}\left(\Delta n_{i}+\Delta n_{i+1}\right)}
\end{aligned}
$$


Discretización hacia atrás:

$$
\left.\frac{\partial \theta_{i}}{\partial n}\right|_{i=N}=C B_{n_{1}} \theta_{i}+C B_{n_{2}} \theta_{i-1}+C B_{n_{3}} \theta_{i-2}=a
$$

Donde:

$$
\begin{aligned}
C B_{n_{1}} & =\frac{\Delta n_{i-2}+2 \Delta n_{i-1}}{\Delta n_{i-1}\left(\Delta n_{i-2}+\Delta n_{i-1}\right)} \\
C B_{n_{2}} & =-\frac{\Delta n_{i-2}+\Delta n_{i-1}}{\Delta n_{i-2} \Delta n_{i-1}} \\
C B_{n_{3}} & =\frac{\Delta n_{i-1}}{\Delta n_{i-2}\left(\Delta n_{i-2}+\Delta n_{i-1}\right)}
\end{aligned}
$$

De igual manera para las condiciones tipo Cauchy, su forma depende del tipo de discretización, para las fronteras ubicadas en $i=1$, se utilizan diferencias hacia delante, mientras que para $i=N$ diferencias hacia atrás (ver Figura 3.3)

Discretización hacia delante:

$$
\left.\frac{\partial \theta_{\lambda}}{\partial n}\right|_{i=1}=C F_{n_{1}} \theta_{i}+C F_{n_{2}} \theta_{i+1}+C F_{n_{3}} \theta_{i+2}=b \theta_{i}+c
$$

Donde el valor de las constantes se especifican en las ecuaciones (3.85 a) - (3.85 b)

Discretización hacia atrás:

$$
\left.\frac{\partial \theta_{i}}{\partial n}\right|_{i=N}=C B_{n_{1}} \theta_{i}+C B_{n_{2}} \theta_{i-1}+C B_{n_{3}} \theta_{i-2}=b \theta_{i}+c
$$

Donde el valor de las constantes se especifican en las ecuaciones (3.86 a) - (3.86 b)

La ecuación de energía también debe satisfacer igualdad de campo y de flux en la interregión, las cuales quedan especificadas por las ecuaciones (2.10a) y (2.10b), y en forma adimensional son:

$$
\begin{array}{ll}
\theta_{\eta}=\theta_{\omega} & \text { en } X=X_{s} \\
\frac{k_{\eta}}{k_{\omega}} \frac{\partial \theta_{\eta}}{\partial X}=-K_{X X} \frac{\partial \theta_{\omega}}{\partial X} & \text { en } X=X_{s}
\end{array}
$$


La discretización en las derivadas de la ecuación anterior dependerán de la fase en la que se evalúa, aplicando discretización hacia atrás para la región fluida y discretización hacia delante en la región porosa:

Aplicando las condiciones interregionales junto con la discretizaciones de las derivadas: se obtiene:

$$
\frac{k_{\eta}}{k_{\omega}} C B_{n_{1}} \theta_{N X I}+C B_{n_{2}} \theta_{N X I-1}+C B_{n_{3}} \theta_{N X I-2}=C F_{n_{1}} \theta_{N X I}+C F_{n_{2}} \theta_{N X I+1}+C F_{N X I+2}
$$

\section{- Condición de frontera para el vector vorticidad}

La discretización para las condiciones frontera de la vorticidad se realizó utilizando una aproximación propuesta por Woods (1954). Para ejemplificar esta discretización se toma como ejemplo la dirección $X$ en la pared.

$$
\zeta_{n}=0, \quad \zeta_{t_{1}}=-\frac{\partial^{2} \Psi_{t_{1}}}{\partial n^{2}}, \quad \zeta_{t_{2}}=-\frac{\partial^{2} \Psi_{t_{2}}}{\partial n^{2}}
$$

De acuerdo con la definición de vorticidad la componente $X$ es:

$$
-\zeta_{X}=\frac{\partial^{2} \Psi_{X}}{\partial X^{2}}+\frac{\partial^{2} \Psi_{X}}{\partial Y^{2}}+\frac{\partial^{2} \Psi_{X}}{\partial Z^{2}}
$$

Donde

$$
\frac{\partial^{2} \Psi_{X}}{\partial Z^{2}}=-\frac{\partial^{2} \Psi_{X}}{\partial X^{2}}-\frac{\partial^{2} \Psi_{X}}{\partial Y^{2}}-\zeta_{X}
$$

Para obtener el valor de la componente correspondiente del vector potencial se realiza una expansión en series de Taylor

$$
\Psi_{X_{2}}=\Psi_{X_{1}} \Delta Z_{1}\left(\frac{\partial \Psi_{X}}{\partial Z}\right)_{1}+\frac{\Delta Z_{1}^{2}}{2}\left(\frac{\partial^{2} \Psi_{X}}{\partial Z^{2}}\right)_{1}+\frac{\Delta Z_{1}^{3}}{6}\left(\frac{\partial^{3} \Psi_{X}}{\partial Z^{3}}\right)_{1}+O\left[\Delta Z_{1}^{4}\right]
$$

Sustituyendo la segunda derivada (3.94) en (3.95) y despejando para $\zeta$ se obtiene:

$$
\begin{aligned}
\zeta_{X_{1}}=\frac{2}{\Delta Z_{1}^{2}}\left(\Psi_{X_{1}}-\Psi_{X_{2}}\right) & +\frac{2}{\Delta Z_{1}}\left(\frac{\partial \Psi_{X}}{\partial Z}\right)_{1}+\frac{\Delta Z_{1}}{3}\left(\frac{\partial^{3} \Psi_{X}}{\partial Z^{3}}\right)_{1} \\
& -\left(\frac{\partial^{2} \Psi_{X}}{\partial X^{2}}\right)_{1}-\left(\frac{\partial^{2} \Psi_{X}}{\partial Y^{2}}\right)_{1}+O\left[\Delta Z_{1}^{2}\right]
\end{aligned}
$$


De la relación entre velocidad y vector potencial se pueden obtener las condiciones de no-desplazamiento de la pared en $Y=0$

$$
\begin{aligned}
& u=\frac{\partial \Psi_{Z}}{\partial Y}-\frac{\partial \Psi_{Y}}{\partial Z}=0 \\
& v=\frac{\partial \Psi_{X}}{\partial Z}-\frac{\partial \Psi_{X}}{\partial Y} \\
& w=\frac{\partial \Psi_{Y}}{\partial X}-\frac{\partial \Psi_{X}}{\partial Y}=0
\end{aligned}
$$

Derivando con respecto a la dirección $Z$ la ecuación (3.94) se obtiene:

$$
\frac{\partial^{3} \Psi_{X}}{\partial Z^{3}}=-\frac{\partial^{3} \Psi_{X}}{\partial X^{3}}-\frac{\partial^{3} \Psi_{X}}{\partial Y^{3}}-\frac{\partial \zeta_{X}}{\partial Z}
$$

Sustituyendo los valores de $\frac{\partial \Psi_{X}}{\partial Z}$ y $\frac{\partial^{3} \Psi_{X}}{\partial Z^{3}}$ de las ecuaciones (3.98) y respectivamente, en la ecuación (3.96) se obtiene:

$$
\begin{aligned}
\zeta_{X_{1}}=\frac{2}{\Delta Z_{1}^{2}}\left(\Psi_{X_{1}}-\Psi_{X_{2}}\right) & +\frac{2}{\Delta Z_{1}}\left(\frac{\partial \Psi_{X}}{\partial Z}\right)_{1}+\frac{\Delta Z_{1}}{3}\left(\frac{\partial^{3} \Psi_{X}}{\partial Z^{3}}\right)_{1} \\
& -\left(\frac{\partial^{2} \Psi_{X}}{\partial X^{2}}\right)_{1}-\left(\frac{\partial^{2} \Psi_{X}}{\partial Y^{2}}\right)_{1}+O\left[\Delta Z_{1}^{2}\right]
\end{aligned}
$$

Las dos últimas derivadas de la ecuación (3.101) se eliminan de acuerdo a

$$
\begin{array}{lc}
\left(\frac{\partial^{2} \Psi_{n}}{\partial n^{2}}\right)=0 & n=X, Y, Z \\
\left(\frac{\partial^{2} \Psi_{X}}{\partial Y^{2}}\right)=0 & \text { Por no desplazamiento }
\end{array}
$$
forma

Despreciando las derivadas de orden superior a 2, la ecuación adquiere la siguiente

$$
\zeta_{X_{1}}=\frac{2}{\Delta Z_{1}^{2}}\left(\Psi_{X_{1}}-\Psi_{X_{2}}\right)+\frac{2}{\Delta Z_{1}}\left(v+\frac{\partial \Psi_{Z}}{\partial X}\right)_{1}+\frac{\Delta Z_{1}}{3}\left(\frac{\partial \zeta_{X}}{\partial Z}\right)+O\left[\Delta Z_{1}^{2}\right]_{1}
$$

Ahora, sustituyendo la definición de derivada hacia adelante en la pared: $\left(\frac{\partial \zeta_{X}}{\partial Z}\right)_{1}=\frac{\zeta_{X_{2}}-\zeta_{X_{1}}}{\Delta Z_{1}}$ y eliminando el término del potencial en la pared se obtiene: 


$$
\zeta_{X_{1}}=-\frac{3}{\Delta Z_{1}^{2}}\left(\Psi_{X_{2}}\right)+\frac{3}{\Delta Z_{1}}\left(v+\frac{\partial \Psi_{Z}}{\partial X}\right)_{1}-\frac{\zeta_{X_{2}}}{2}
$$

El resumen de la secuencia de evaluación de las ecuaciones y condiciones de frontera se muestra en la Figura 3.5

\subsection{ESTABILIDAD Y CONVERGENCIA}

En la deducción y solución de las ecuaciones se generaron diferentes tipos de parámetros, entre ellos los relacionados con el método del falso estado transitorio, factores de relajación en el esquema numérico, entre los más importantes y aunque se ha demostrado que el método del falso estado transitorio es estable para la solución del sistemas de ecuaciones, los valores del incremento del tiempo tienen gran influencia en la velocidad de convergencia. Por ejemplo Leonardi (1984) sugiere para un sistema en dos dimensiones con una sola región un valor del incremento expresado mediante $\Delta t \leq 0.8[\min (\Delta X, \Delta Y)]^{2}$, cuando los valores de $\alpha_{\zeta}=\alpha_{\theta}=1$.

Para sistemas de ecuaciones acopladas el valor del incremento del tiempo así como los valores de las constantes del método del falso estado transitorio quedan relacionadas entre sí, y los valores reportados proveen de estabilidad pero no de rapidez de convergencia. En lo que respecta a los parámetros de relajación para las ecuaciones de vorticidad, potencial y temperatura se cuentan también con algunos valores reportados. Sin embargo, al utilizar dos regiones los valores de los parámetros cambian ya que las constantes están fuertemente relacionadas, por lo que es muy conveniente determinar la combinación de los valores de estas constantes con el fin de incrementar la velocidad en la convergencia. Las pruebas que muestran la dependencia de estos parámetros se reportaron anteriormente (Valencia, 1993).

En cuanto a la convergencia se dice que se alcanza en el momento en que se satisface un determinado criterio, entonces en el método del falso estado transitorio se tiene el estado estable cuando el siguiente criterio de convergencia para cualquier variable satisface la siguiente expresión:

$$
\frac{1}{M_{X} M_{Y} M_{Z}} \sum_{i=1}^{i=M_{X}} \sum_{j=1}^{j=M_{Y}} \sum_{k=1}^{k=M_{Z}}\left|\Delta \varphi_{i j k}^{n+1}-\Delta \varphi_{i j k}^{n}\right| \leq \text { error }_{\varphi}
$$




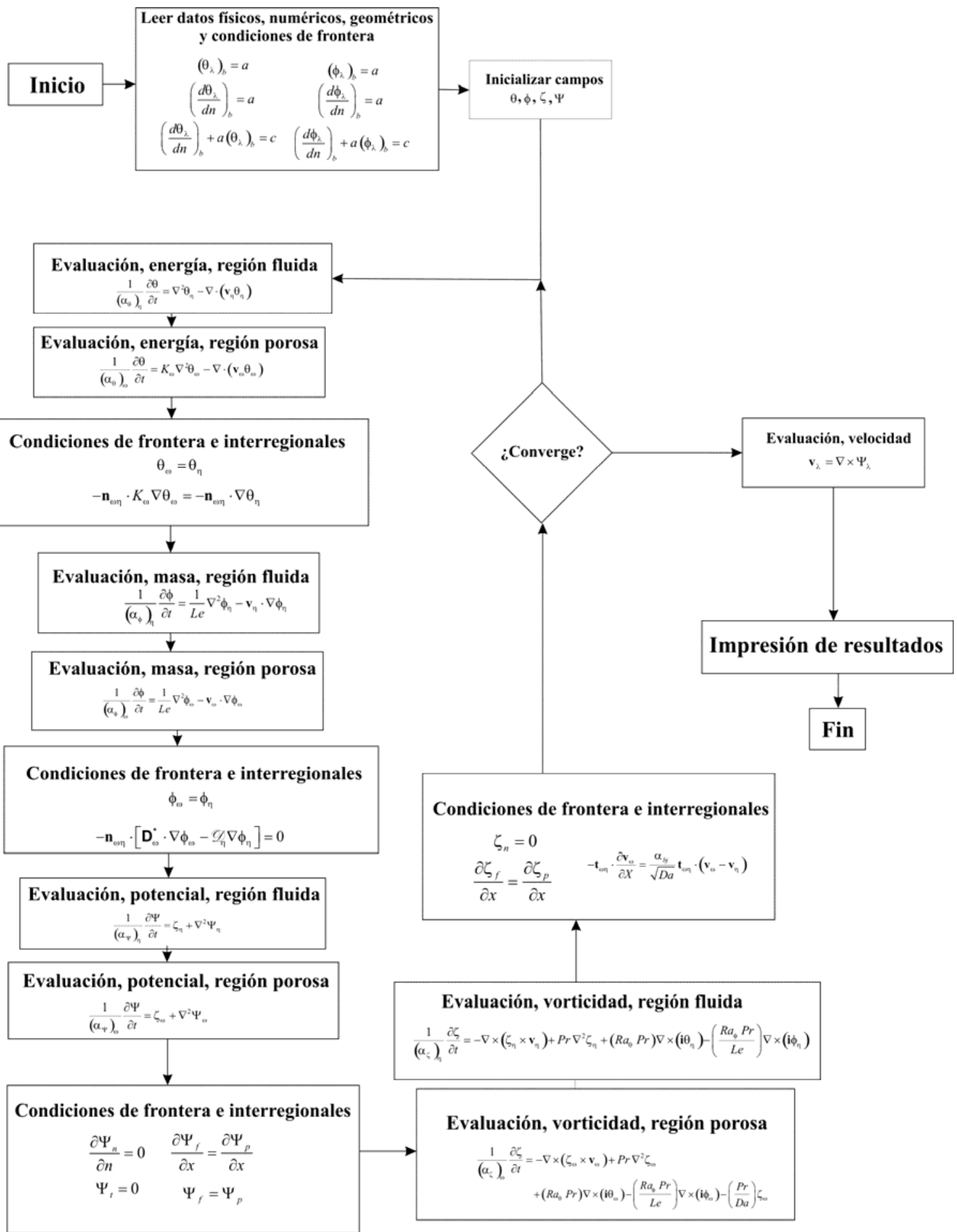

Figuras 3.5.- Secuencia de evaluación de las ecuaciones. 
No obstante, en algunos casos se utiliza el criterio de convergencia basado en el error relativo tomando en cuenta el efecto de la variable con respecto al tiempo artificial (Mallinson y de Vahl Davis, 1973):

$$
\frac{\frac{1}{M_{X} M_{Y} M_{Z}} \sum_{i=1}^{i=M_{X}} \sum_{j=1}^{j=M_{Y}} \sum_{k=1}^{k=M_{Z}}\left|\frac{\Delta \varphi_{i j k}^{n+1}-\Delta \varphi_{i j k}^{n}}{\Delta t}\right|}{\left|\Delta \varphi_{i j k}^{n}\right|_{\max }} \leq \text { error }_{\varphi}
$$

Donde $M_{k}$ indica el número de nodos en la dirección $k, \Delta \varphi_{i, j, k}^{N}$ es la corrección al campo obtenido con el método ADI después de haber realizado el barrido en las tres direcciones en la iteración $N$, y $\varphi_{\max }^{N}$ es el valor máximo del campo después de la iteración $N$. Este criterio de convergencia fue impuesto a todas las variables en la región fluida y porosa, estos son: vector potencial, vector vorticidad, temperatura y concentración. 


\section{Capítulo 4}

\section{Pruebas e interpretación de resultados.}

En un sistema consistente de una cavidad, con una fase fluida en la que se presenta flujo convectivo provocado por diferencias de temperatura y concentración, el gran número de posibilidades de flujo está dado por el número (relativamente grande) de parámetros adimensionales que gobiernan el fenómeno, entre ellos el número de Rayleigh $(R a)$, el número de Lewis $(L e)$, la relación de flotación $(N)$ y la relación de longitudes entre otros, en el caso de un sistema con dos regiones se requiere considerar adicionalmente los parámetros que involucran la permeabilidad del medio poroso $(D a)$, relación de conductividades $(R c)$ etc., lo anterior implica la realización de un gran número de pruebas para analizar el modelo completo; En el presente capítulo se presentan sólo algunas de las pruebas realizadas, en las cuales los cambios muestran efectos importantes, la elección de las condiciones se tomó considerando los trabajos publicados con anterioridad, por ejemplo, en el caso tridimensional se utilizaron las condiciones utilizadas por Singh y col. (1993).

Para simplificar el número de pruebas a realizar, el flujo convectivo bajo consideración fue dividido en dos clases: a) flujo por fuerzas debidas a la transferencia de calor, en donde los efectos de flotación son dominados por los cambios de temperatura y b) fuerzas debidas a la transferencia de masa, donde los efectos de flotación son controlados por los cambios de densidad asociados con los cambios de concentración; al primer grupo se le realizarán pruebas a los modelos en dos y tres dimensiones, mientras que las del segundo grupo sólo al modelo en dos dimensiones. Para cada uno de los modelos tridimensionales se analizaron los siguientes niveles para cada variable: 
Con el fin de presentar la validación de los modelos y de analizar los efectos que muestran en la interregión se realizaron una serie de pruebas a los modelos desarrollados, los resultados se compararon con los obtenidos por el modelo tridimensional que considera la corrección de Brinkman a la ecuación de Darcy para modelar la transferencia de cantidad de movimiento (modelo presentado por Singh y col.,1993), para el caso bidimensional se desarrolló un modelo, el cual se comparó con los resultados obtenidos previamente por diversos autores entre los que se encuentran: Nishimura y col.(1986), el desarrollo del modelo bidimensional se presenta en el Apéndice C.

\begin{tabular}{|c|c|c|c|c|}
\hline$R a$ & $D a$ & $R c$ & Interregión & Tamaño de la malla \\
\hline \hline $10^{4}$ & $10^{-3}$ & 1 & 0.00 & $11^{3}$ \\
\hline $10^{5}$ & $10^{-4}$ & 5 & 0.25 & $21^{3}$ \\
\hline $10^{6}$ & & 10 & 0.50 & $41^{3}$ \\
\cline { 1 - 3 } & & & 0.75 & $81^{3}$ \\
\cline { 3 - 5 } & & & 1.00 & \\
\hline
\end{tabular}

Tabla 4.1 Niveles de los parámetros analizados en el caso tridimensional

\subsection{Condiciones de frontera.}

Como ya se mencionó en el Capítulo 2, el modelo fue desarrollado para manejar diferentes condiciones de frontera, sin embargo gran parte de las pruebas se adaptaron a las condiciones previamente presentadas por otros autores, por ejemplo, para el caso tridimensional Singh y col.(1993) consideran los planos $X$ y $Z$ adiabáticos y la diferencia de temperatura fue fijada entre los planos $Y=0$ y $Y=1$, lo anterior para analizar los efectos de las fuerzas de flotación, estas condiciones se muestran de manera esquemática en la Figura 4.1, para la mayoría de las pruebas presentadas en este trabajo se utilizaron estas condiciones, lo anterior para poder realizar las comparaciones entre modelos en tres dimensiones, en el caso de modelos bidimensionales se utilizan las condiciones adecuadas dependiendo de la prueba y del trabajo con el que se compara. 


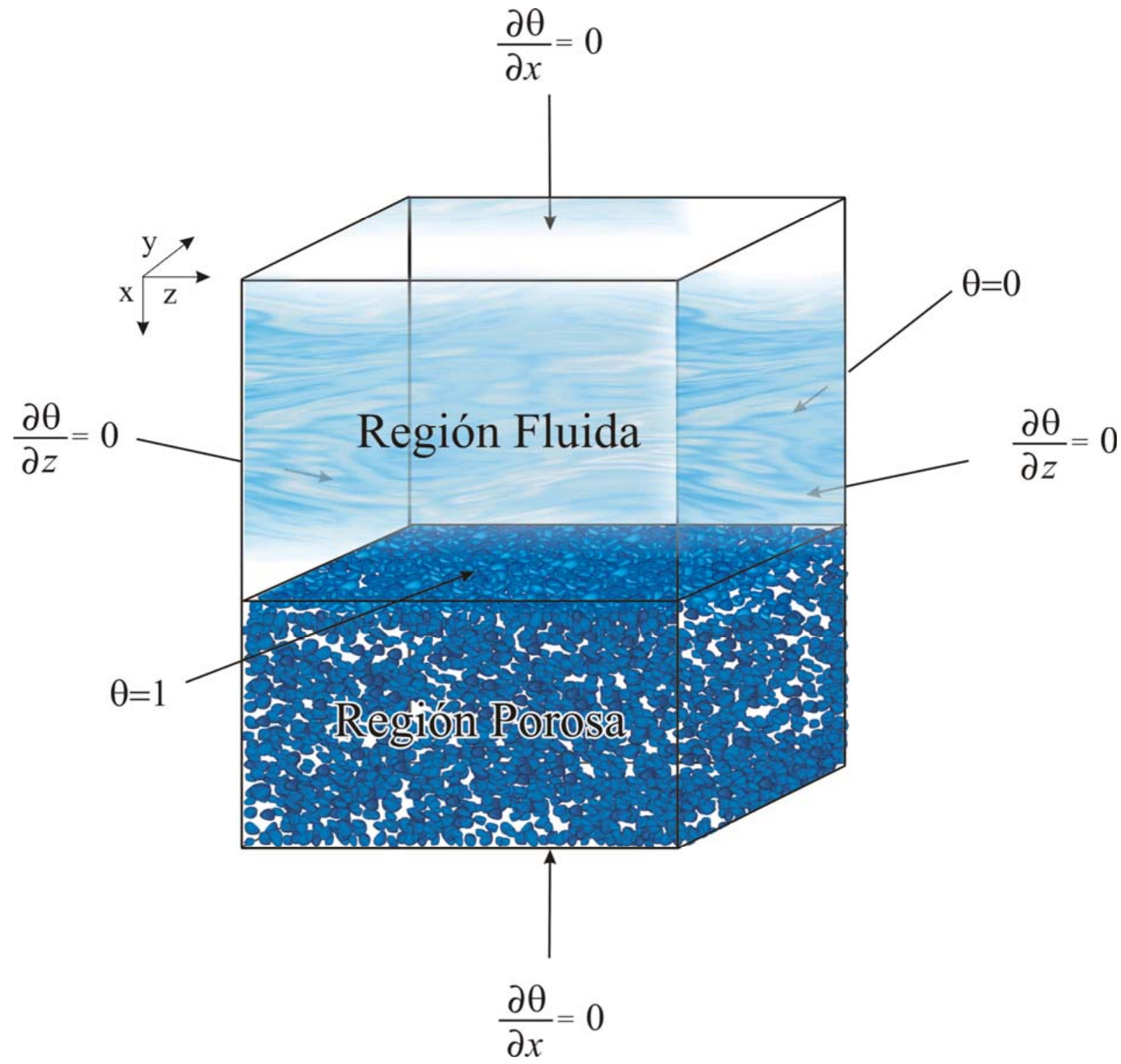

Figura 4.1.- Condiciones de frontera para la ecuación de energía. 


\subsection{Parámetros numéricos.}

En la solución numérica se debe de tener cuidado al establecer diferentes parámetros propios del método, por ejemplo, al incluir el falso transitorio se debe de establecer un valor para el incremento del tiempo artificial $(\Delta t)$ éste tiene una fuerte dependencia con el tamaño mínimo del espaciamiento de la malla, además debe de ser suficientemente grande para asegurar estabilidad en la convergencia y rapidez para encontrar la solución; en el presente trabajo se utiliza la expresión presentada por Singh y col. (1993) para determinar el tamaño de $\Delta t$ (ver sección 3.5).

Otro conjunto de parámetros que influyen en la estabilidad del método son los relacionados con el método del falso transitorio, estos incluyen valores para las variables temperatura, concentración, vector vorticidad y vector potencial para cada una de las regiones (Valencia, 1993). Los valores que se utilizaron para las condiciones de frontera mostradas en la Figura 4.1 se presenta en la Tabla 4.2:

\begin{tabular}{|l|c|c|}
\cline { 2 - 3 } \multicolumn{1}{c|}{} & Región Fluida & Medio Poroso \\
\hline$\alpha_{\theta}$ & 5.0 & 5.0 \\
\hline$\alpha_{\phi}$ & 5.0 & 5.0 \\
\hline$\alpha_{\zeta}$ & 0.1 & 2.5 \\
\hline$\alpha_{\Psi}$ & 5.0 & 25 \\
\hline
\end{tabular}

Tabla 4.2 Valores de los parámetros para el método de falso estado transitorio en ambas regiones.

Sin embargo, estos valores tienen una dependencia de diversos parámetros físicos y numéricos tales como espaciamiento y tamaño de la malla computacional e incremento en el número de Rayleigh ( $R a_{\theta}$ y/o $R a_{\phi}$ ), para la mayoría de las pruebas se utilizaron los parámetros mostrados en la Tabla 4.2, en algunos casos se mejoró la velocidad de convergencia modificando el valor de uno o varios parámetros durante el proceso iterativo, sin embargo el objetivo del trabajo radica en encontrar los valores que muestren estabilidad numérica, un estudio sobre los efectos de estos parámetros sobre la velocidad de convergencia se presentó previamente (Valencia 1993), concluyendo que aunque el método es estable, para cierto conjunto de valores de los parámetros se presenta un comportamiento asintótico y periódico en la convergencia. 
Para escribir el programa de cómputo (MURPHY 3D) se utilizó el lenguaje FORTRAN 90 y para mostrar la variación del tiempo de cómputo requerido por los modelos de Brinkman en comparación con el que utiliza las condiciones de Beavers y Joseph, en la Tabla 4.3 se muestran los resultados obtenidos utilizando una computadora con procesador Pentium IV a 3.2 GHz, Los tiempos de ejecución se reportan para las condiciones de frontera mostradas en la Figura 4.1 con una relación de conductividades $R c=1$ y haciendo variar el tamaño de malla, $R a, D a$ y modelo tridimensional utilizado, como se mencionó anteriormente el número de iteraciones para llegar a la convergencia depende fuertemente de los parámetros del método del falso estado transitorio, para las pruebas que se presentan se utilizaron los valores reportados en la Tabla 4.2.

En la Tabla 4.3 se muestra como a tamaños de malla pequeño, el tiempo de cómputo es muy corto y similar en ambos casos, lo anterior sin importar que se tenga una diferencia en el número de iteraciones, sin embargo, a medida que se incrementa el tamaño de malla el modelo que utiliza las condiciones empíricas de Beavers y Joseph requieren de un mayor número de iteraciones, lo anterior es consecuencia del acoplamiento en ambas regiones, sobre todo por la convergencia de las ecuaciones de vorticidad y que muestran sus efectos en los mapas de contorno mostrados para la componente $Z$ del vector potencial (ver Figura 4.11), en este punto se puede concluir que el modelo de Brinkman presenta un mejor comportamiento en cuanto a la convergencia y tiempo de cómputo requerido, los resultados de las variables se mostraran posteriormente.

Para mostrar el efecto que tiene la elección de los parámetros del método del falso estado estacionario en la convergencia del modelo computacional, se realizaron pruebas con una cavidad cúbica tomando un $R a=10^{6}$, y un $D a=10^{-3}$, la mitad inferior de la cavidad ocupada por el medio poroso, las condiciones de frontera son las especificadas por la Figura 4.1, el espaciamiento es constante para una malla de $41^{3}$. Los resultados se muestran en la Tabla 4.4 en función de las iteraciones y del tiempo de cómputo, para la variación de los parámetros de la ecuación de la vorticidad y potencial, se eligieron las variables anteriores por ser las que muestran mayor sensibilidad a la convergencia, la tabla muestra como los valores para la región fluida utilizados en la ecuación de la vorticidad muestran gran variación en el tiempo de convergencia para un rango de 0.1-0.5, en contraste, los valores para la región porosa tiene poca influencia inclusive para un rango de 2.5-40 la diferencia en el número de iteraciones para alcanzar la convergencia es mínima. 


\begin{tabular}{|c|c|c|c|c|c|c|}
\hline \multirow{2}{*}{$\begin{array}{l}\text { Puntos } \\
\text { en la } \\
\text { malla }\end{array}$} & \multirow[b]{2}{*}{$\mathbf{R a}$} & \multirow[b]{2}{*}{ Da } & \multicolumn{2}{|c|}{ Brinkman } & \multicolumn{2}{|c|}{$\begin{array}{l}\text { Beavers y Joseph con } \\
\qquad \alpha L_{x} / \sqrt{P_{\gamma \omega}}=1.0\end{array}$} \\
\hline & & & Iteraciones & $\begin{array}{c}\text { Tiempo CPU } \\
\text { (seg) }\end{array}$ & Iteraciones & $\begin{array}{c}\text { Tiempo CPU } \\
\text { (seg) }\end{array}$ \\
\hline $11^{3}$ & $10^{4}$ & $10^{-4}$ & 3,120 & 11 & 2,727 & 10 \\
\hline $21^{3}$ & $10^{4}$ & $10^{-4}$ & 2,537 & 89 & 2,450 & 83 \\
\hline $41^{3}$ & $10^{4}$ & $10^{-4}$ & 2,315 & 825 & 2,352 & 814 \\
\hline $81^{3}$ & $10^{4}$ & $10^{-4}$ & 2,184 & 8,833 & 3,489 & 13,877 \\
\hline $11^{3}$ & $10^{4}$ & $10^{-3}$ & 3,133 & 12 & 2,774 & 10 \\
\hline $21^{3}$ & $10^{4}$ & $10^{-3}$ & 2,566 & 90 & 2,487 & 84 \\
\hline $41^{3}$ & $10^{4}$ & $10^{-3}$ & 2,370 & 843 & 2,385 & 825 \\
\hline $81^{3}$ & $10^{4}$ & $10^{-3}$ & 2,286 & 9,242 & 3,525 & 14,021 \\
\hline $11^{3}$ & $10^{5}$ & $10^{-4}$ & 2,283 & 8 & 1,759 & 7 \\
\hline $21^{3}$ & $10^{5}$ & $10^{-4}$ & 1,777 & 62 & 1,479 & 50 \\
\hline $41^{3}$ & $10^{5}$ & $10^{-4}$ & 1,592 & 568 & 1,348 & 466 \\
\hline $81^{3}$ & $10^{5}$ & $10^{-4}$ & 1,525 & 6,181 & 1,976 & 7,933 \\
\hline $11^{3}$ & $10^{5}$ & $10^{-3}$ & 2,397 & 8 & 1,784 & 6 \\
\hline $21^{3}$ & $10^{5}$ & $10^{-3}$ & 2.081 & 73 & 1,672 & 56 \\
\hline $41^{3}$ & $10^{5}$ & $10^{-3}$ & 1,871 & 664 & 1,561 & 540 \\
\hline $81^{3}$ & $10^{5}$ & $10^{-3}$ & 1,762 & 7,120 & 1,962 & 7,876 \\
\hline
\end{tabular}

Tabla 4.3 Iteraciones y tiempos de cómputo para diferentes $R a, D a$ y tamaño de malla.

De igual manera los valores del parámetro para la ecuación del vector potencial toman importancia en la región fluida, el rango utilizado para este parámetro se encuentra entre 1-20, el valor de este parámetro en conjunto con el de la vorticidad determinan la velocidad de convergencia, para las condiciones que se están analizando la combinación que ofrece el mínimo número de iteraciones es 0.5 , 20 para la vorticidad y vector potencial respectivamente con 26 iteraciones, y la combinación con un mayor número de iteraciones es 0.4, 1 con 3276 para un mismo conjunto de condiciones. 


\begin{tabular}{|c|c|c|c|c|c|}
\hline \multicolumn{2}{|c|}{ Vorticidad } & \multicolumn{2}{|c|}{ Potencial } & \multirow[t]{2}{*}{ Iteraciones } & \multirow{2}{*}{$\begin{array}{l}\text { Tiempo de } \\
\text { Cómputo } \\
\text { (seg) }\end{array}$} \\
\hline$\left(\alpha_{\zeta}\right)_{\eta}$ & $\left(\alpha_{\zeta}\right)_{\omega}$ & $\left(\alpha_{\Psi}\right)_{\eta}$ & $\left(\alpha_{\Psi}\right)_{\omega}$ & & \\
\hline 0.1 & 2.5 & 1 & 25 & 1422 & 134 \\
\hline 0.1 & 2.5 & 1 & 40 & 1422 & 133 \\
\hline 0.1 & 2.5 & 5 & 25 & 1309 & 123 \\
\hline 0.1 & 2.5 & 5 & 40 & 1309 & 123 \\
\hline 0.1 & 2.5 & 10 & 25 & 1331 & 124 \\
\hline 0.1 & 2.5 & 10 & 40 & 1331 & 123 \\
\hline 0.1 & 40 & 1 & 25 & 1422 & 135 \\
\hline 0.1 & 40 & 1 & 40 & 1422 & 134 \\
\hline 0.1 & 40 & 5 & 25 & 1309 & 125 \\
\hline 0.1 & 40 & 5 & 40 & 1309 & 125 \\
\hline 0.1 & 40 & 10 & 25 & 1331 & 123 \\
\hline 0.1 & 40 & 10 & 40 & 1331 & 123 \\
\hline 0.25 & 2.5 & 1 & 25 & 1308 & 121 \\
\hline 0.25 & 2.5 & 1 & 40 & 1308 & 121 \\
\hline 0.25 & 2.5 & 5 & 25 & 512 & 47 \\
\hline 0.25 & 2.5 & 5 & 40 & 512 & 48 \\
\hline 0.25 & 2.5 & 10 & 25 & 519 & 48 \\
\hline 0.25 & 2.5 & 10 & 40 & 519 & 49 \\
\hline 0.4 & 2.5 & 1 & 25 & 3276 & 304 \\
\hline 0.4 & 2.5 & 1 & 40 & 3276 & 322 \\
\hline 0.4 & 2.5 & 5 & 25 & 862 & 83 \\
\hline 0.4 & 2.5 & 5 & 40 & 862 & 83 \\
\hline 0.4 & 2.5 & 10 & 25 & 352 & 34 \\
\hline 0.4 & 2.5 & 10 & 40 & 352 & 34 \\
\hline 0.5 & 2.5 & 6 & 25 & 4569 & 447 \\
\hline 0.5 & 2.5 & 6 & 40 & 4569 & 467 \\
\hline 0.5 & 2.5 & 10 & 25 & 686 & 68 \\
\hline 0.5 & 2.5 & 10 & 40 & 686 & 67 \\
\hline 0.5 & 2.5 & 20 & 25 & 268 & 26 \\
\hline 0.5 & 2.5 & 20 & 40 & 268 & 26 \\
\hline
\end{tabular}

Tabla 4.4.- Efecto de los parámetros del método del falso estado transitorio en la convergencia y tiempo de cómputo. 
Para contar con gráficas que muestren la variación en los perfiles de temperatura, concentración, componentes del vector potencial y del vector velocidad, los resultados se presentan en forma de superficies y mapas de contorno tomando una sección transversal ubicada en la parte media de eje $Z$ tal y como se muestra en la Figura 4.2, quedando la superficie y/o mapa de contorno sobre los ejes $X$ y $Y$, esto ayuda de igual manera a tener un monitoreo de la interregión y los efectos que el acoplamiento de ecuaciones tiene en ambas regiones, adicionalmente se presentan perfiles bidimensionales ubicados a lo largo del eje $X$ en la parte media del eje $Y$ del mapa de contorno. Además, ya que los planos comprendidos entre $X=0$ hasta $X=1$ y $Z=0$ hasta $Z=1$ son adiabáticos y el gradiente de temperatura es impuesta entre los planos $Y=0$ y $Y=1$, las variables físicas cambian de forma significativamente en la dirección $Y$ y en la dirección $X$ como resultado de las fuerzas de flotación, por esta razón los mapas de contorno son reportados en el plano $Z=0.5$ para el caso tridimensional y comparados con aquellos calculados en el modelo en dos dimensiones, en los cuales se ha utilizado el tamaño de espaciamiento constante.

\subsection{Efecto de los Números de Rayleigh y Darcy}

El objetivo de la prueba que considera la variación de los números de Rayleigh y Darcy es analizar los efectos sobre el perfil de temperatura, velocidad y vector potencial para verificar de manera cualitativa las tendencias en el régimen laminar, los resultados reportados en esta sección son para una cavidad cúbica con la mitad inferior del volumen llena de medio poroso.

Los resultados que se reportan con el nombre de modelo de "Brinkman" coinciden con los obtenidos con la formulación presentada por Singh y col. 1993, se omite la comparación por no mostrar diferencias.

En la Figura 4.3 se muestran los mapas de contorno para la temperatura, en ellos se muestran simultáneamente los resultados para el modelo que utiliza la corrección de Brinkman a la ecuación de Darcy, con líneas continuas y el modelo que utiliza la condición de Beavers y Joseph con líneas segmentadas. 


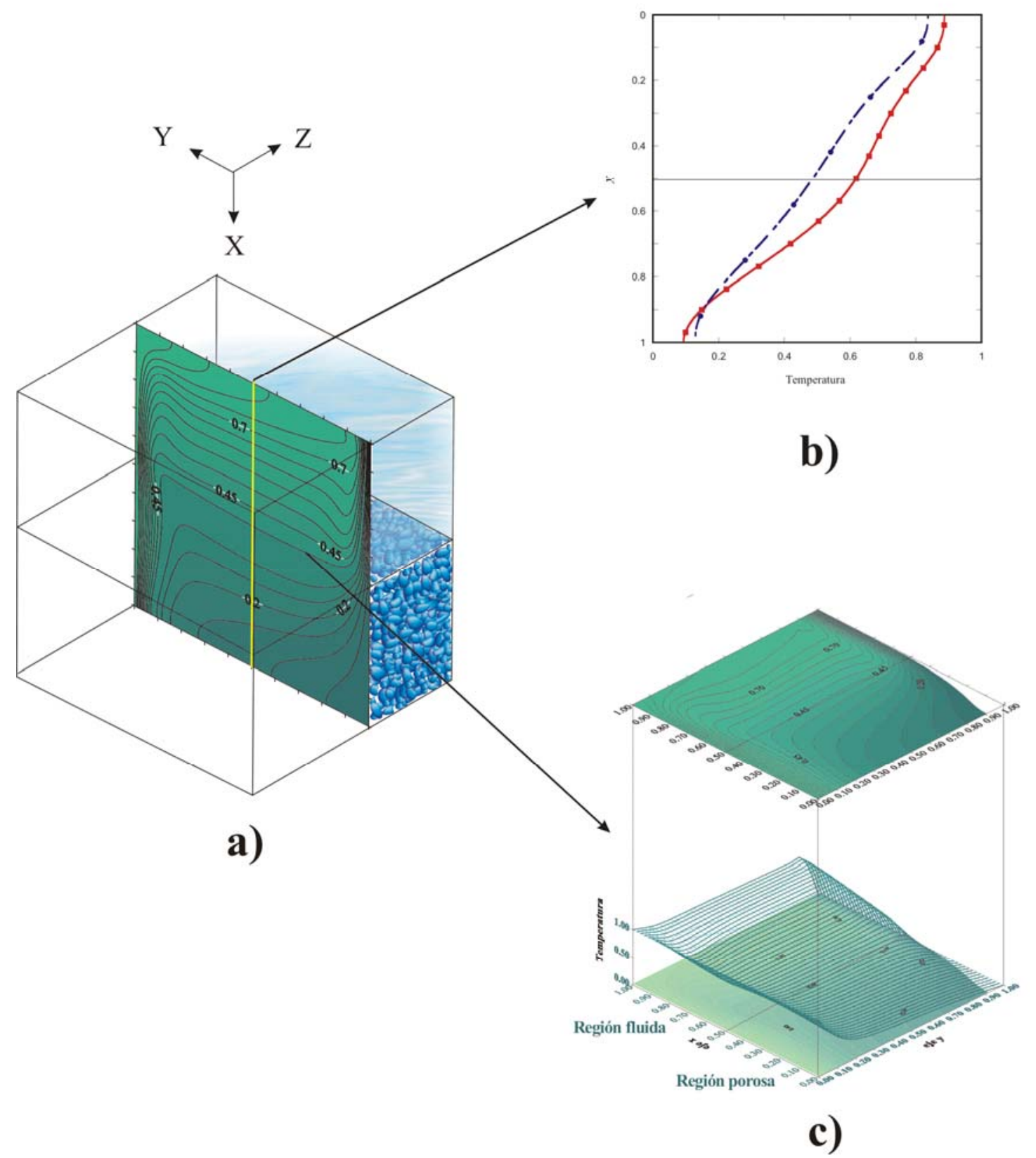

Figura 4.2.- Superficies y mapas de contorno para las pruebas del modelo tridimensional. 
Respecto a las isotermas se puede observar que la convección natural comienza a ser importante conforme aumenta el Número de Rayleigh se incrementa de $10^{4}$ a $10^{6}$, esto se hace evidente por la inclinación de las isotermas y el tamaño entre las líneas al acercarse a las paredes, sobre todo en la región fluida, una vista comparativa de las figuras indican que las isotermas son más densas cerca de las paredes caliente y fría a $R a=10^{5}$ y $10^{6}$ como una consecuencia del incremento de las fuerzas de flotación asociadas con el incremento del $R a$, y que en la región porosa se tiene una contribución conductiva a bajos Rayleigh. De igual manera para el efecto del Número de Darcy, al incrementarse de $10^{-4}$ a $10^{-3}$ se puede observar que los efectos de la convección natural también comienzan a hacerse significantivos en la región porosa, notándose que para un Número de Darcy determinado, la velocidad del flujo que penetra desde la región fluida a la región porosa queda en función del Número de Rayleigh.

En cuanto a la comparación entre los modelos de Brinkman y las condiciones de Beaver y Joseph se puede observar que para valores bajos de $R a$ las isotermas son iguales en ambas regiones, sin embargo conforme se aumenta el $\mathrm{Da}$ los efectos muestran diferencias, sobre todo en la región porosa, lo anterior por los efectos de la permeabilidad. El caso más drástico se tiene para $R a=10^{6}, D a=10^{-3}$ en la que los efectos de permeabilidad en la región porosa y de términos de flotación en la región fluida son de gran importancia.

La isoterma 0.8 para el modelo de Beavers y Joseph muestra un máximo a la distancia 0.19 , este valor también se observa en la isoterma 0.7 para el modelo de Brinkman, este efecto se observa por los fuertes efectos convectivos en la reión fluida muy cercanos a la pared caliente, a valores de $\mathrm{Ra}=10^{6}$ se presentan efectos marcados en las cercanías con la pared caliente comenzando a presentar valores máximos para las isotermas de la región fluida, este punto se muestra claramente en la Figura 4.4.

En la Figura 4.4 se presenta una comparación de los modelos en tres dimensiones en los planos $Y=0.25$ y $Y=0.5$ para la ecuación de energía, se tomaron las condiciones extremas de $R a=10^{6}$ y $D a=10^{-3}$ para mostrar las isotermas, en la figura se puede observar como no existen diferencias entre el mismo modelo para los diferentes planos, sin embargo la diferencia entre modelos es muy notoria sobre todo en las partes cercanas a la interregión. 

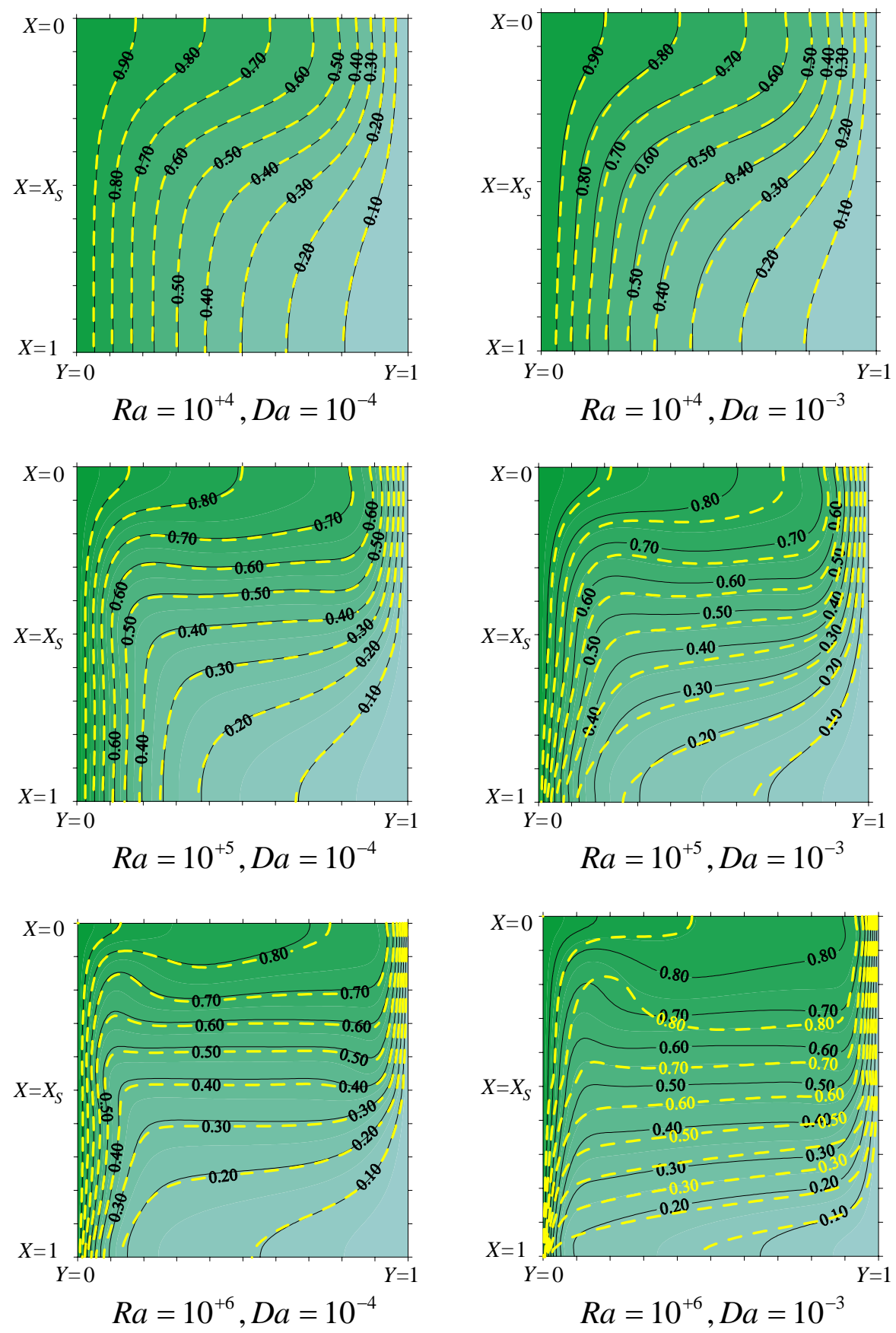

Figura 4.3.- Mapas de contorno de temperatura modelos tridimensionales: - - ByJ; Brinkman, Xs=0.5. 
En la Figura 4.5 se presenta la comparación de la temperatura para los modelos en dos y tres dimensiones mostrando isotermas a $Y=0.25$, en ella se observa que las diferencia se notan entre los modelos mientras que estas diferencia son mínimas para la misma formulación sin importar las dimensiones. Las diferencias más notorias se ubican cerca de la interregión, mientras que en las paredes los valores de temperatura son muy cercanos, lo anterior nuevamente hace notar que para valores altos de Rayleigh y Darcy las formulaciones muestran gran discrepancia en la cercanía de la interregión, esto indica que las diferencias no son afectadas por el hecho de resolver el modelo en tres dimensiones sino por la forma de acoplar las ecuaciones de transferencia de cantidad de movimiento, lo anterior sugiere un análisis más detallado en esta región, en estas situaciones es donde las condiciones de salto son útiles para definir con más formalidad los efectos interregionales.

La comparación en tres dimensiones para las condiciones de $R a$ y $D a$ antes mencionadas se muestran en la Figura 4.6, en ella se nota como a $Y=0$ los perfiles de temperatura muestran valores mayores para el modelo que considera la ecuación de Beavers y Joseph para el acoplamiento de la ecuación de cantidad de movimiento ya que depende del valor del parámetro de Beavers y Joseph.

El valor que en la figura 4.3 aparece como un máximo en las isotermas 0.8 y 0.7 para los modelos de Beavers-Joseph y Brinkman respectivamente, en la Figura 4.4 se presenta como un punto de inflexión a un valor de 0.18 en la posición del eje $\mathrm{X}$, este efecto es causa de la influencia convectiva en la región fluida cerca de la pared caliente.

La mayor diferencia entre modelos se encuentra en la interregión mientras que al mismo tiempo este punto representa la diferencia mínima para el mismo modelo en posición diferente, la diferencia en la interregión es consecuencia de la forma de acoplar las ecuaciones, en el caso del modelo de Beavers y Joseph esta diferencia puede ser reducida al modificar el valor del parámetro semiempírico, sin embargo esto no garantiza el comportamiento en la región fluida, 


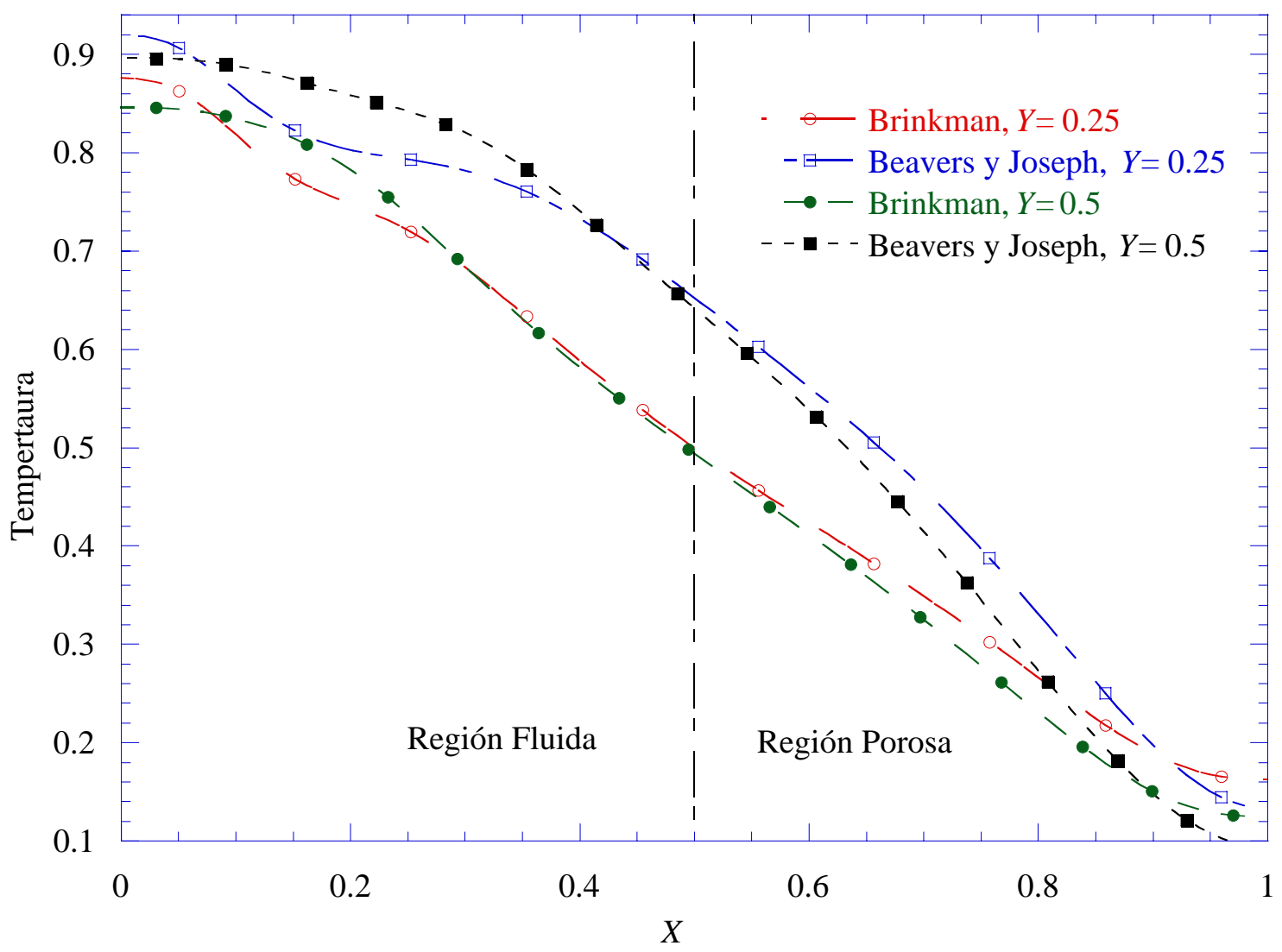

Figura 4.4.- Comparación de los perfiles de temperatura a $Y=0.25$ y 0.5, Modelos en 3D, Rayleigh $10^{6}$, Darcy $10^{-3}$. 


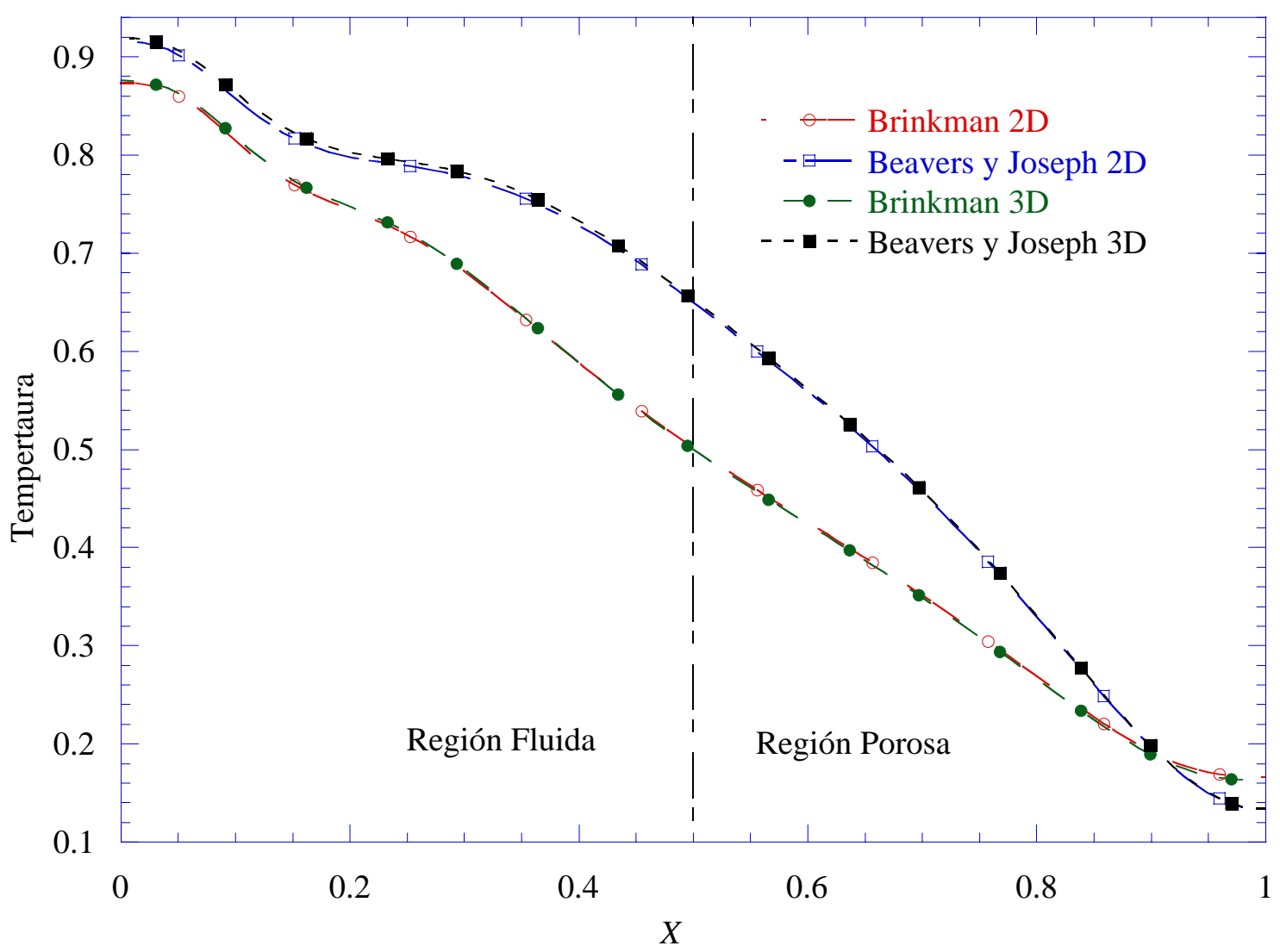

Figura 4.5.- Comparación de los perfiles de temperatura a $Y=0.25$, Modelos en 2D y 3D, Rayleigh $10^{6}$, Darcy $10^{-3}$. 


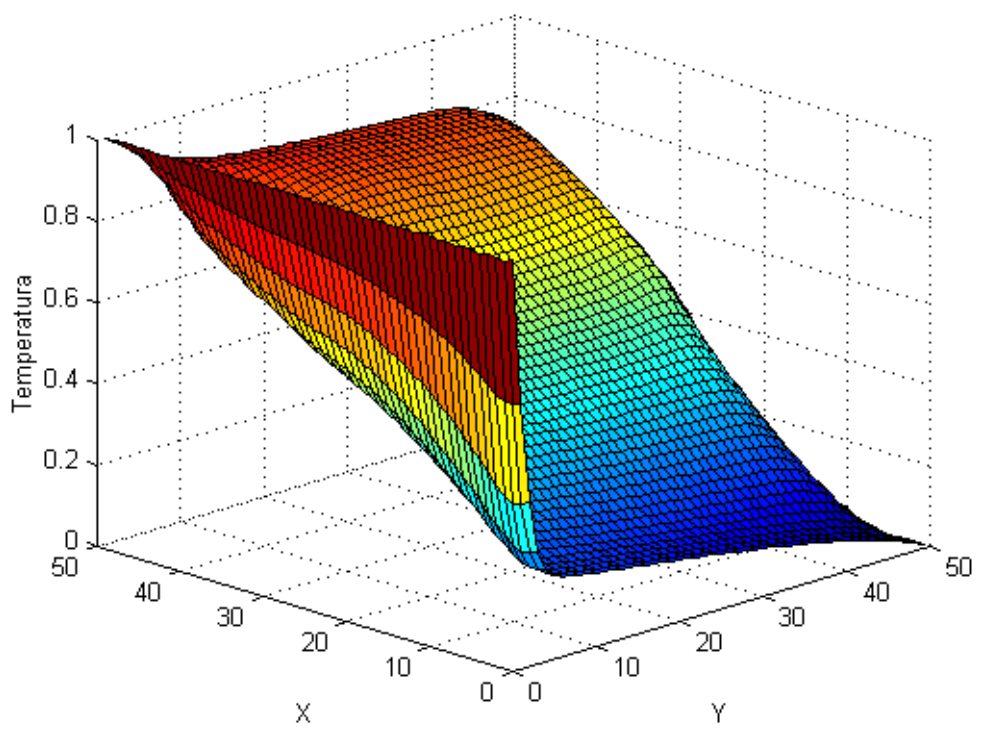

a) Corrección de Brinkman a la ecuación de Darcy

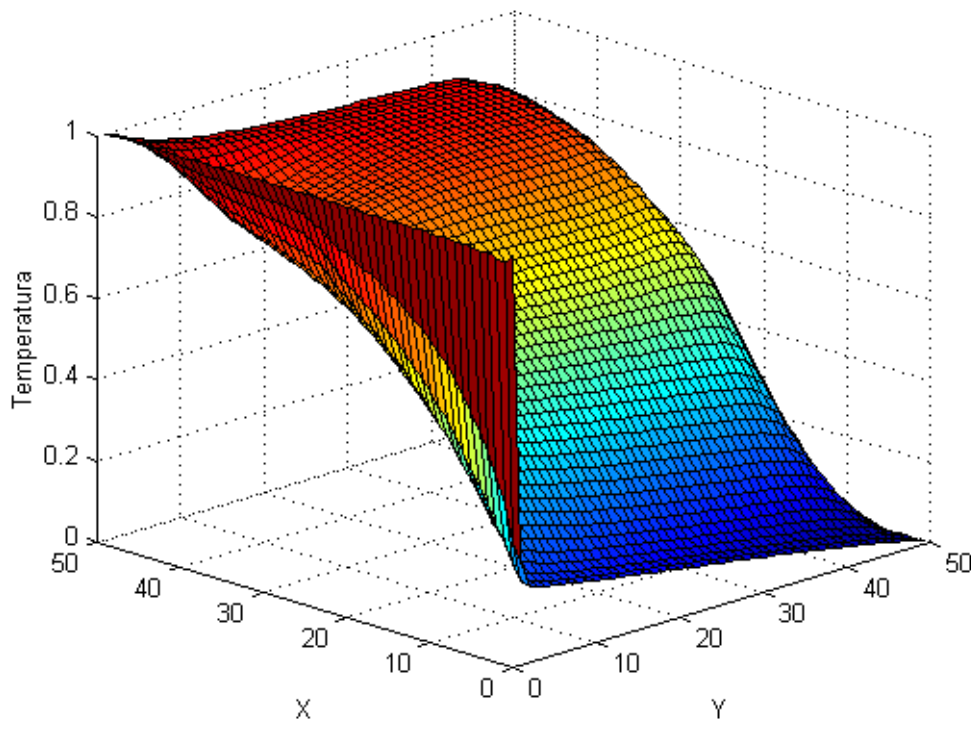

b) Condiciones de Beavers y Joseph en la interregión

Figura 4.6.- Superficies, comparación de los perfiles de temperatura para los modelos de a) Brinkman y b) B y J. 


\subsubsection{Número de Nusselt.}

Desde el punto de vista práctico, resulta de gran interés la evaluación de la velocidad de transferencia de calor resultado de las diferencias de temperatura entre las paredes fría y caliente. En este trabajo los efectos de transferencia de calor son reportados en términos del número de Nusselt para las paredes fría y caliente mostradas en la Figura 4.1, teniéndose las siguientes definiciones

Número de Nusselt (promedio lateral)

$$
\left.\overline{N u}_{X}(X)\right|_{Y^{*}}=\left.\frac{L_{X}}{L_{Z}} \int_{0}^{L_{Z} / L_{X}} N u(X, Z)\right|_{Y^{*}} d Z
$$

Número de Nusselt (promedio global)

$$
\left.\overline{N u}\right|_{Y^{*}}=\left.\frac{L_{X}}{L_{Z}} \int_{0}^{1} \int_{0}^{L_{Z} / L_{X}} N u(X, Z)\right|_{Y^{*}} d Z d X
$$

En donde el subíndice $Y^{*}$ indica la evaluación en $Y=0$ o $Y=L_{Y} / L_{X}$, y $N u(X, Z)$ indica el Número de Nusselt local en las paredes fría y caliente para cualquier región, y está dado por:

$$
N u(X, Z)=\frac{h L_{X}}{k_{\gamma}}=\left\{\begin{array}{ccc}
-\frac{\partial \theta_{\eta}}{\partial Y}, & \text { para } & 0 \leq X<X_{s} \\
-\frac{1}{2}\left(\frac{\partial \theta_{\eta}}{\partial Y}+K_{\omega} \frac{\partial \theta_{\omega}}{\partial Y}\right), & \text { a } & X=X_{s} \\
-K_{\omega} \frac{\partial \theta_{\omega}}{\partial Y}, & \text { para } & X_{s}<X \leq 1
\end{array}\right.
$$



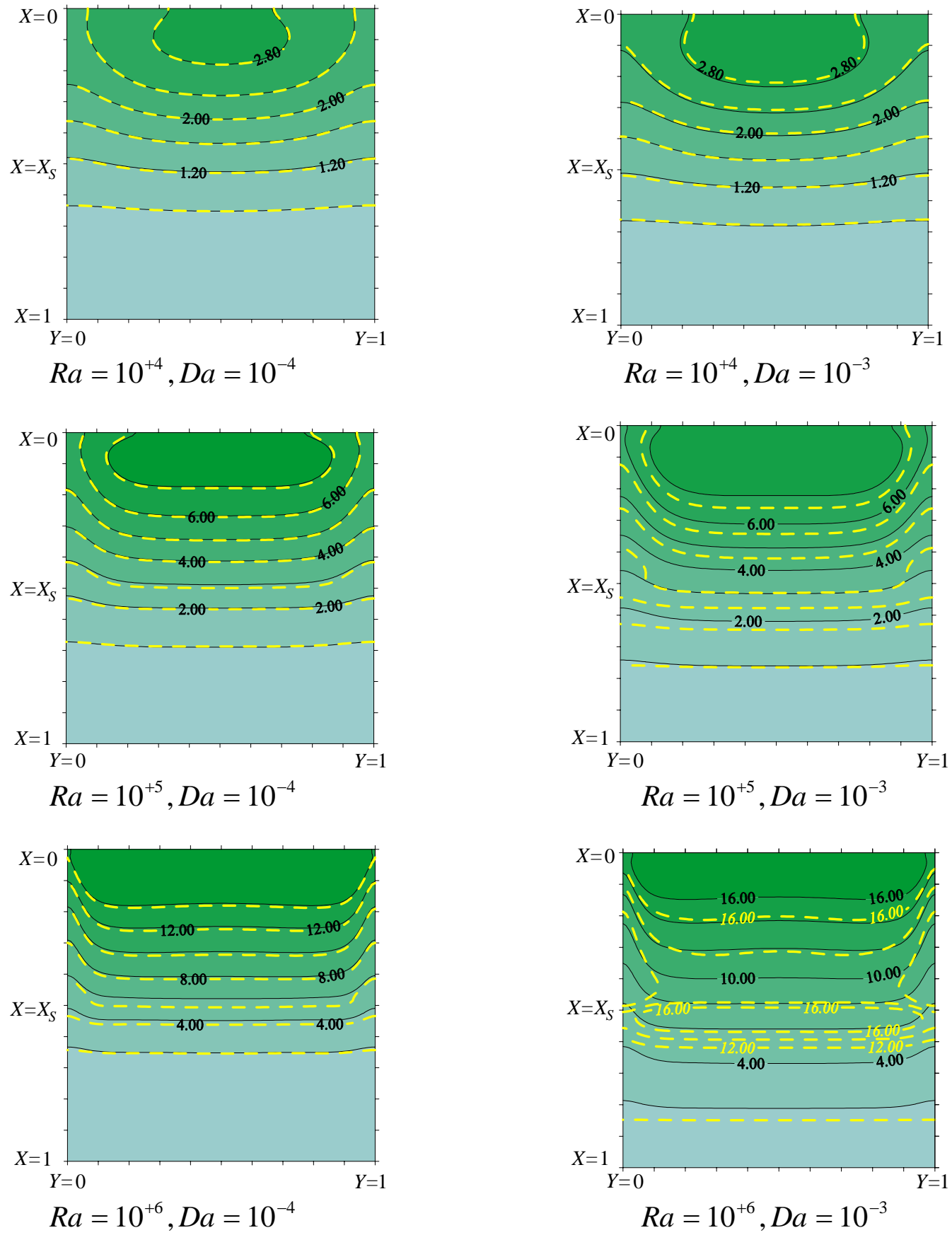

Figura 4.7.- Mapas de contorno para el Número de Nusselt: modelos tridimensionales: ByJ.; Brinkman, $X_{\mathrm{S}}=0.5$. 
La Figura 4.7 muestra simultáneamente los efectos al variar los números de Rayleigh y Darcy, sobre el número de Nusselt. El valores del $N u(X, Z)$ es usado como parámetro para estimar la velocidad de transferencia de calor local, los valores en el plano $Y=1$ se muestra en la Figura 4.7. En ella se observa que cuando el Número de Rayleigh $=10^{04}$ el Número de Nusselt local en el medio poroso tiende a incrementarse conforme el Darcy se incrementa, esto es de esperarse ya que el flujo del fluido es mas vigoroso en esta región. En contraste, el número de Nusselt es en la región fluida relativamente insensible al número de Darcy, indicando que el mecanismo de transferencia de calor en el medio poroso no afecta la región fluida, lo anterior no es tan sorprendente, ya que anteriormente se había hecho notar que la conducción juega un papel dominante a bajos números de Rayleigh. Cuando el número de Rayleigh se ubica entre $10^{4}$ y $10^{5}$, el número de Nusselt local es mas alto, y la fase fluida contribuye significativamente a la transferencia de calor.

Los efectos sobre el Nusselt promedio se muestran en las figuras 4.8 y 4.9, en ellas se puede observar que el $\left.\overline{N u}_{X}(X)\right|_{Y^{*}}$ decrece en la región fluida y se incrementa en la región porosa conforme se incrementa el $D a$. En el caso cuando $R a=10^{+04}$ el fenómeno conductivo es más importante, aquí los valores de $\left.\overline{N u}_{X}(X)\right|_{Y^{*}}$ son más uniformes. En la pared fría $Y=1$, los campos de flujo resultan por el espaciamiento mayor de los perfiles de temperatura, aquí los valores altos del $\left.\overline{\mathrm{Nu}}_{X}(X)\right|_{\mathrm{Y}^{*}}$ se encuentran a valores altos del Número de Rayleigh altos valores del número de Rayleigh, y cuando el $D a=10^{-03}$, es Nusselt es mucho más grande que cuando el $D a$ es $10^{-4}$ y $10^{-5}$. 

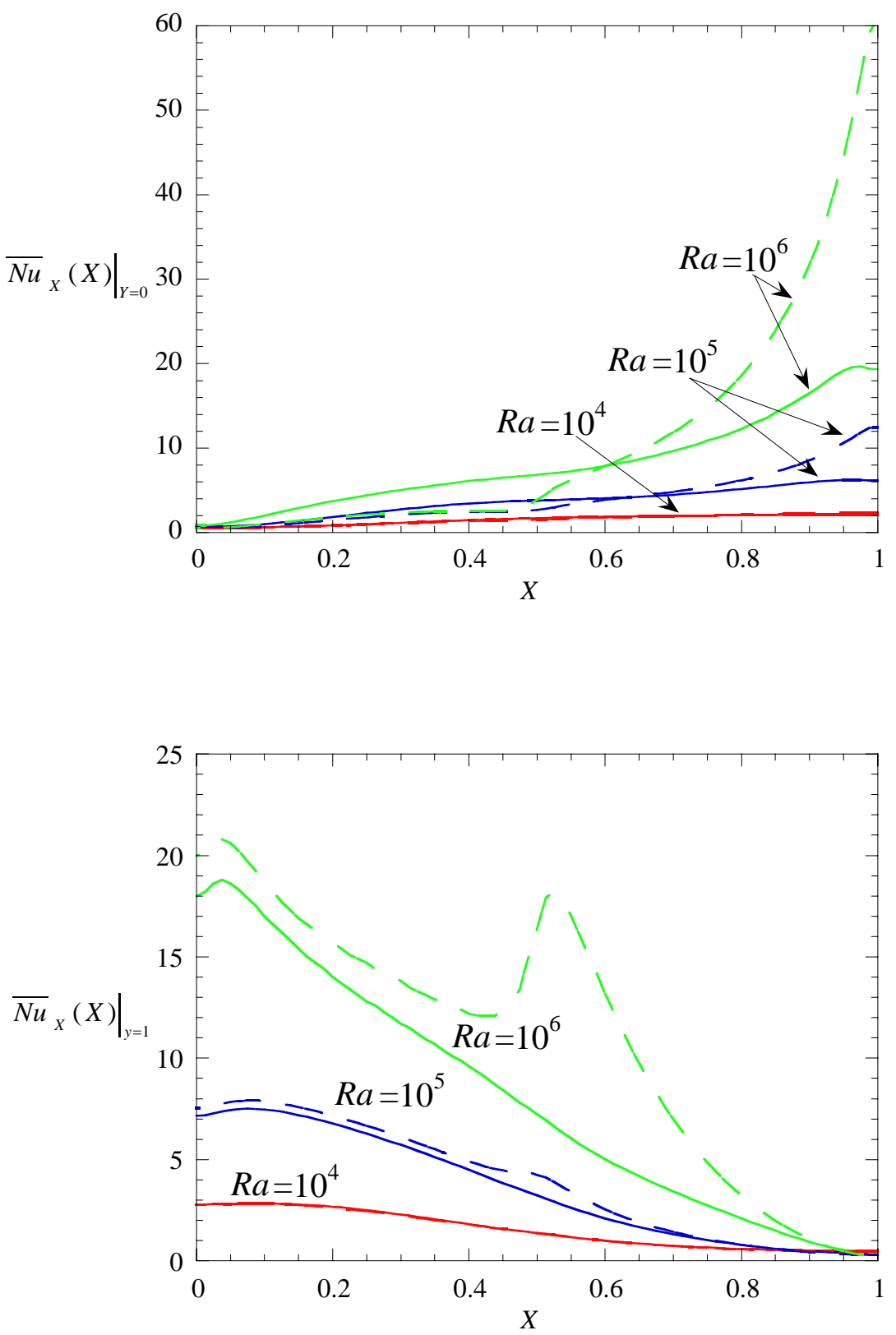

b)

Figura 4.8.- Comparación del número de Nusselt local en la pared fría, obtenida mediante a)Brinkman y b) B y J: $R a=10^{6}, D a=10^{-3}$, parámetro de Beavers y Joseph = 1.0 

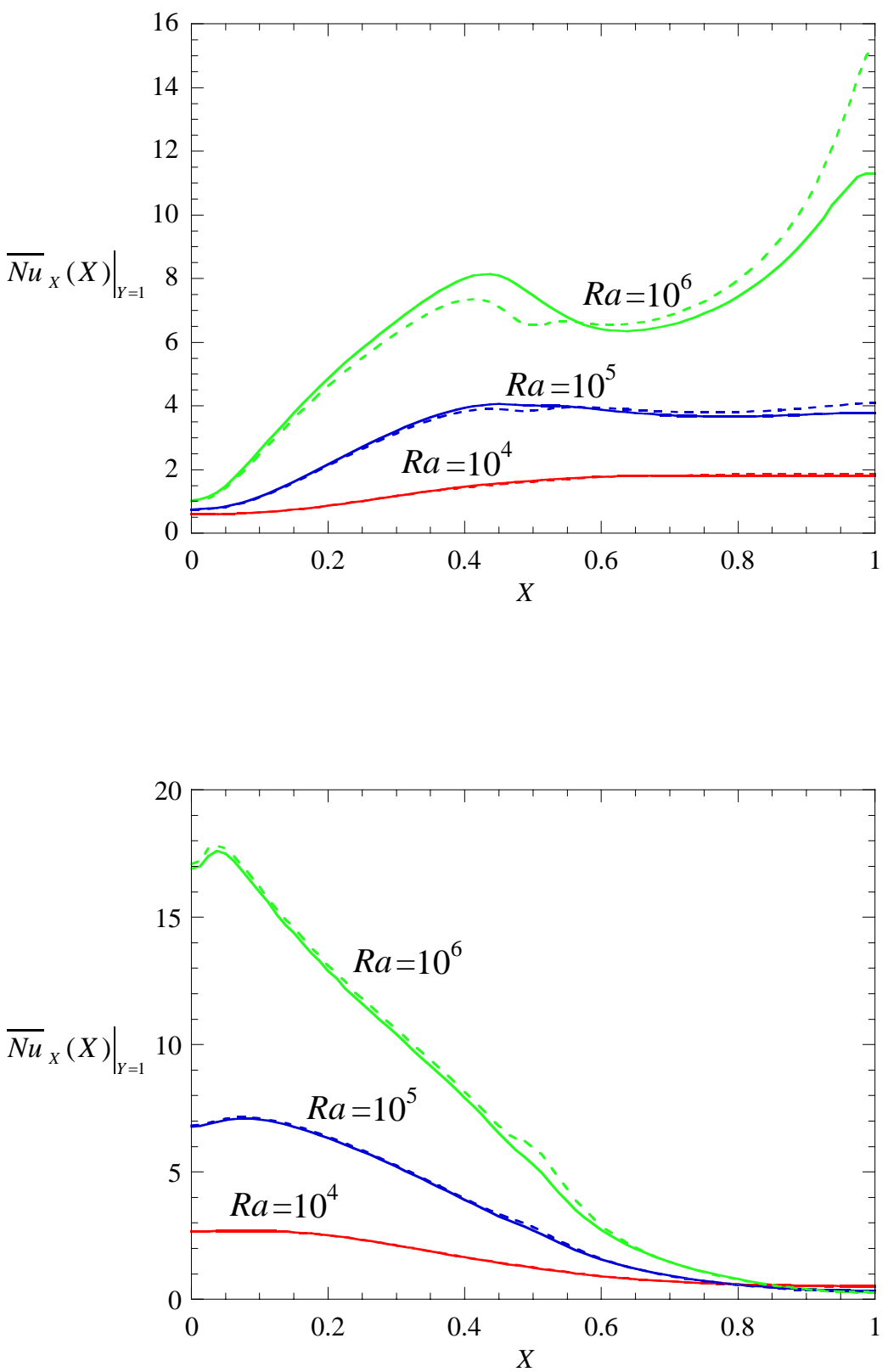

Figura 4.9.- Comparación del número de Nusselt local en la pared fría a)Brinkman b) B y J: $D a=10^{-3}$, parámetro de Beavers y Joseph $=1.0$ 

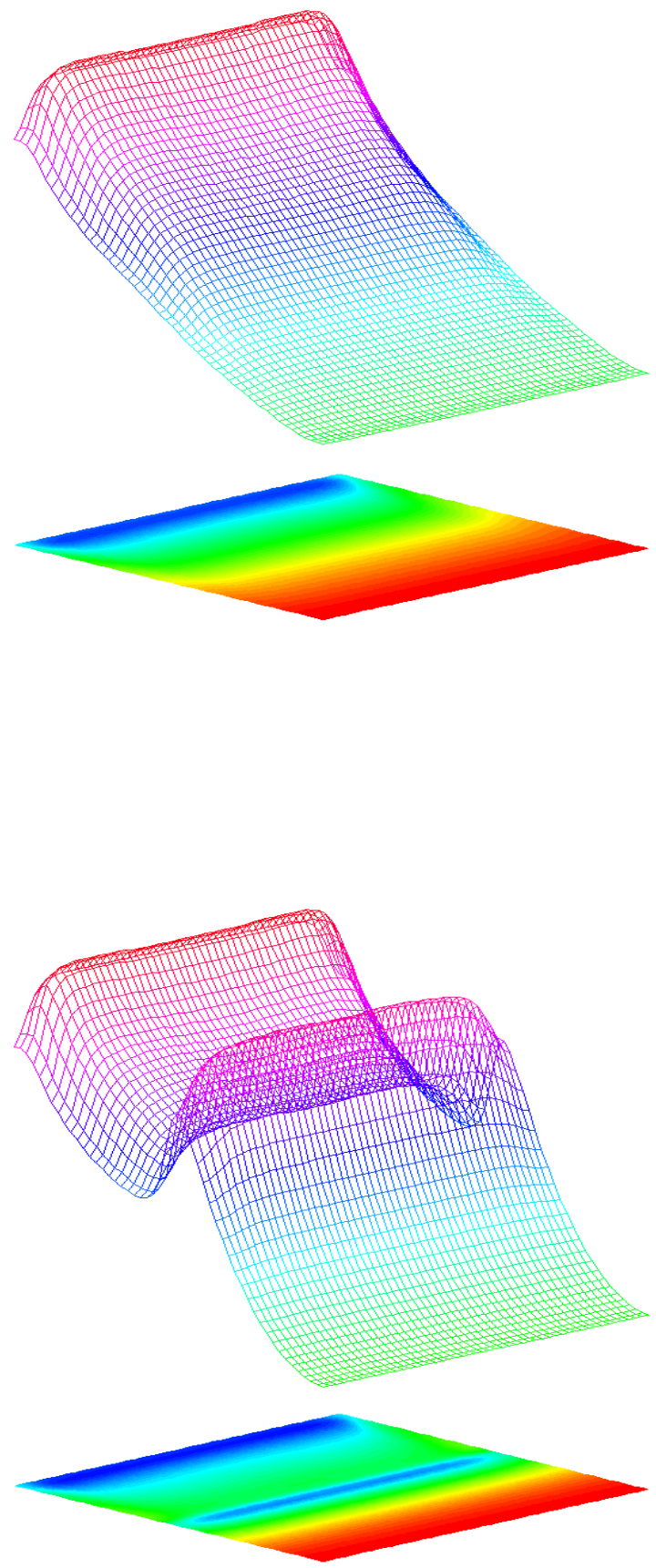

Figura 4.10.- Superficie y mapa de contorno para la componente $Z$ de la velocidad $(w)$ modelos tridimensionales: - - B y J; Brinkman; $X \mathrm{~s}=0.5, R c=1.0$ 
La convergencia del método numérico se muestra en la Tabla 4.5 en términos del número de Nusselt promedio de las paredes fría y caliente, para cada valor de $D a$ y $R a$, el número de Nusselt en ambas paredes tiene una tendencia al valor reportado en la columna "adoptado”. El número de Nusselt que se tomó para la comparación entre las aproximaciones de Darcy y Brinkman y para la prueba de exactitud del balance de calor promedio fue obtenido por una extrapolación lineal del número de Nusselt usando el inverso del tamaño de malla como variable independiente. Como se indica en la Tabla 4.5, para la extrapolación a $R a=10^{+6}$ fueron usados sólo el número de Nusselt calculado para $41^{3}$ y $81^{3}$. La comparación del Nusselt promedio indica que las diferencias entre las predicciones para la situación con $R a$ $=10^{6}$ y $D a=10^{-3}$ con ambos tipos de malla es $3.3 \%$. Esta diferencia es mucho más pequeña que la obtenida entre la malla que utiliza espaciamiento constante obtenidos del modelo que utiliza el modelo de Darcy con la extensión de Brinkman (4.6\%) reportado en la tabla, por tanto, la precisión de los resultados mostrados en la Tabla 4.1 son suficientes para el propósito de comparar ambas aproximaciones del modelado de la transferencia de cantidad de movimiento en la región porosa.

Los resultados de la Tabla 4.5 muestran que para cualquier valor establecido del $R a$ y Da, el balance global de energía en el sistema es satisfecho con un error menor al 2.2\%, lo anterior debido a que el Nusselt promedio de las paredes fría y caliente tienen una tendencia al mismo valor conforme se incrementa el número de puntos en la malla.

En la Tabla 4.6 se presenta la comparación del número de Nusselt promedio obtenido en el presente trabajo respecto al reportado por Singh y col. 1993 las diferencias son mínimas lo que lleva a concluir la fuerte concordancia con los resultados en el modelo que utiliza la corrección de Brinkman a la ecuación de Darcy en comparación con la formulación de Beavers y Joseph la cual tiene la dependencia de los parámetros semiempíricos. 


\begin{tabular}{|c|c|c|c|c|c|c|c|c|}
\hline \multirow{2}{*}{$\begin{array}{l}\text { Puntos } \\
\text { en la } \\
\text { malla }\end{array}$} & \multirow[t]{2}{*}{$\mathrm{Ra}$} & \multirow[t]{2}{*}{$\mathrm{Da}$} & \multicolumn{3}{|c|}{ Brinkman } & \multicolumn{3}{|c|}{$\begin{array}{c}\text { Beavers y Joseph con } \\
\alpha L_{x} / \sqrt{P_{\gamma \omega}}=1.0\end{array}$} \\
\hline & & & Caliente & Frío & Adoptado & Caliente & Frío & Adoptado \\
\hline
\end{tabular}

\begin{tabular}{|c|c|c|c|c|c|c|c|c|}
\hline $11^{3}$ & $10^{4}$ & $10^{-4}$ & $1.42^{*}$ & 1.78 & & $1.26^{*}$ & $1.62^{*}$ & \\
\hline $21^{3}$ & $10^{4}$ & $10^{-4}$ & $1.42 *$ & $1.60 *$ & & $1.36^{*}$ & $1.54^{*}$ & \\
\hline $41^{3}$ & $10^{4}$ & $10^{-4}$ & $1.42 *$ & $1.50 *$ & & $1.40 *$ & $1.48 *$ & \\
\hline $81^{3}$ & $10^{4}$ & $10^{-4}$ & $1.42 *$ & $1.45^{*}$ & & $1.42 *$ & $1.46^{*}$ & \\
\hline$\infty$ & $10^{4}$ & $10^{-4}$ & 1.42 & 1.40 & 1.42 & 1.45 & 1.44 & 1.45 \\
\hline $11^{3}$ & $10^{4}$ & $10^{-3}$ & 1.51 & $1.83^{*}$ & & 1.34 & 1.67 & \\
\hline $21^{3}$ & $10^{4}$ & $10^{-3}$ & $1.52 *$ & $1.66 *$ & & $1.45^{*}$ & $1.60 *$ & \\
\hline $41^{3}$ & $10^{4}$ & $10^{-3}$ & $1.52 *$ & $1.57 *$ & & $1.48^{*}$ & $1.54 *$ & \\
\hline $81^{3}$ & $10^{4}$ & $10^{-3}$ & $1.52 *$ & $1.54 *$ & & $1.50 *$ & $1.52 *$ & \\
\hline$\infty$ & $10^{4}$ & $10^{-3}$ & 1.52 & 1.49 & 1.51 & 1.52 & 1.49 & 1.51 \\
\hline $11^{3}$ & $10^{5}$ & $10^{-4}$ & 3.62 & 3.83 & & 3.28 & 3.59 & \\
\hline $21^{3}$ & $10^{5}$ & $10^{-4}$ & $3.34^{*}$ & $3.66 *$ & & 3.25 & 3.59* & \\
\hline $41^{3}$ & $10^{5}$ & $10^{-4}$ & $3.20 *$ & $3.35 *$ & & $3.19 *$ & $3.34^{*}$ & \\
\hline $81^{3}$ & $10^{5}$ & $10^{-4}$ & $3.17 *$ & $3.22 *$ & & $3.20 *$ & $3.26^{*}$ & \\
\hline$\infty$ & & & 3.10 & 3.07 & 3.09 & 3.21 & 3.14 & 3.18 \\
\hline $11^{3}$ & $10^{5}$ & $10^{-3}$ & 4.02 & 4.10 & & 3.76 & 3.98 & \\
\hline $21^{3}$ & $10^{5}$ & $10^{-3}$ & $3.81 *$ & $4.00 *$ & & 3.92 & $4.16^{*}$ & \\
\hline $41^{3}$ & $10^{5}$ & $10^{-3}$ & $3.64 *$ & $3.72 *$ & & 3.93* & $4.00 *$ & \\
\hline $81^{3}$ & $10^{5}$ & $10^{-3}$ & $3.59 *$ & $3.62 *$ & & $3.93 *$ & $3.94 *$ & \\
\hline$\infty$ & $10^{5}$ & $10^{-3}$ & 3.51 & 3.48 & 3.50 & 3.93 & 3.86 & 3.90 \\
\hline $11^{3}$ & $10^{6}$ & $10^{-4}$ & 6.26 & 5.45 & & 6.17 & 5.13 & \\
\hline $21^{3}$ & $10^{6}$ & $10^{-4}$ & $7.45 *$ & 7.30 & & 7.30 & 7.18 & \\
\hline $41^{3}$ & $10^{6}$ & $10^{-4}$ & $6.85 *$ & $7.11^{*}$ & & $6.86^{*}$ & $7.17^{*}$ & \\
\hline $81^{3}$ & $10^{6}$ & $10^{-4}$ & $6.54 *$ & $6.67 *$ & & $6.70 *$ & $6.84^{*}$ & \\
\hline$\infty$ & $10^{6}$ & $10^{-4}$ & 6.24 & 6.23 & 6.24 & 6.54 & 6.51 & 6.53 \\
\hline $11^{3}$ & $10^{6}$ & $10^{-3}$ & 6.56 & 6.60 & & 6.39* & 6.78 & \\
\hline $21^{3}$ & $10^{6}$ & $10^{-3}$ & 8.74 & 8.73 & & $8.85^{*}$ & 9.92 & \\
\hline $41^{3}$ & $10^{6}$ & $10^{-3}$ & $8.48 *$ & 8.53* & & $10.2^{*}$ & $11.1^{*}$ & \\
\hline $81^{3}$ & $10^{6}$ & $10^{-3}$ & $8.05 *$ & $8.07 *$ & & $11.0^{*}$ & $11.2^{*}$ & \\
\hline$\infty$ & $10^{6}$ & $10^{-3}$ & 7.62 & 7.61 & 7.62 & 11.5 & 11.3 & 11.4 \\
\hline
\end{tabular}

Tabla 4.5 Efecto del número de Rayleigh, Darcy y tamaño de la red sobre el número de Nusselt promedio de las paredes fría y caliente obtenidos con los modelos de Darcy y con la corrección de Brinkman 


\begin{tabular}{|c|c|c|c|c|}
\hline \multirow[b]{2}{*}{$\begin{array}{l}\text { Puntos } \\
\text { en la } \\
\text { malla }\end{array}$} & \multirow[b]{2}{*}{$R a$} & \multirow[b]{2}{*}{$D a$} & \multicolumn{2}{|c|}{ Nusselt promedio } \\
\hline & & & $\begin{array}{l}\text { Singh } y \\
\text { col. } 1993\end{array}$ & $\begin{array}{l}\text { Presente } \\
\text { trabajo }\end{array}$ \\
\hline
\end{tabular}

\begin{tabular}{|l|l|l|l|l|}
\hline $11^{3}$ & $10^{4}$ & $10^{-3}$ & 1.605 & 1.67 \\
\hline $21^{3}$ & $10^{4}$ & $10^{-3}$ & 1.556 & 1.59 \\
\hline & & & & \\
\hline $11^{3}$ & $10^{4}$ & $10^{-4}$ & 1.524 & 1.60 \\
\hline $21^{3}$ & $10^{4}$ & $10^{-4}$ & 1.468 & 1.51 \\
\hline $11^{3}$ & $10^{5}$ & $10^{-3}$ & 4.105 & 4.06 \\
\hline $21^{3}$ & $10^{5}$ & $10^{-3}$ & 3.844 & 3.90 \\
\hline & & & & \\
\hline $11^{3}$ & $10^{5}$ & $10^{-4}$ & 3.774 & 3.72 \\
\hline $21^{3}$ & $10^{5}$ & $10^{-4}$ & 3.408 & 3.50 \\
\hline & & & & \\
\hline $11^{3}$ & $10^{6}$ & $10^{-4}$ & 6.146 & 5.86 \\
\hline $21^{3}$ & $10^{6}$ & $10^{-4}$ & 7.526 & 7.35 \\
\hline & & & & \\
\hline
\end{tabular}

Tabla 4.6 Comparación del Nusselt promedio con los reportados con Singh y col. 1993

En la Figura 4.11 se muestran los mapas de contorno para las líneas de corriente (componente “Z” del vector potencial), en los mapas de contorno se muestran simultáneamente los resultados para el modelo que utiliza la corrección de Brinkman a la ecuación de Darcy (con líneas continuas) y el modelo que utiliza la condición de Beavers y Joseph (líneas segmentadas), de esta manera se puede realizar una comparación simultánea entre ambos modelos para dos números adimensionales.

Respecto a las líneas de corriente, se puede observar al igual que para las isotermas, la convección natural comienza a ser fuerte al aumentar el número de Rayleigh (pasando de $10^{4}$ a $10^{6)}$, esto se hace evidente con la deformación de la célula (mínimo) en la región fluida, llegando al grado de tener dos células asimétricas para un $R a$ de $10^{6} \mathrm{y} \mathrm{Da} 10^{-3}$, una comparación de las figuras indican que las líneas de corriente son más densas cerca de las paredes caliente y fría a $R a=10^{5}$ y $10^{6}$ como una consecuencia del incremento de las fuerzas de flotación asociadas con el incremento del $R a$; y que en la región porosa se tiene una contribución conductiva a bajos $R a$, esto se hace patente al presentar el máximo en la región fluida. De igual manera para el efecto del Número de Darcy, al incrementarse de $10^{-4}$ a $10^{-3}$ se puede observar que los efectos de la convección natural también comienzan a hacerse significativos en la región porosa. Notándose que para un Número de Darcy fijo, la velocidad del flujo que penetra desde la región fluida a la región porosa queda en función del Número de Rayleigh. 

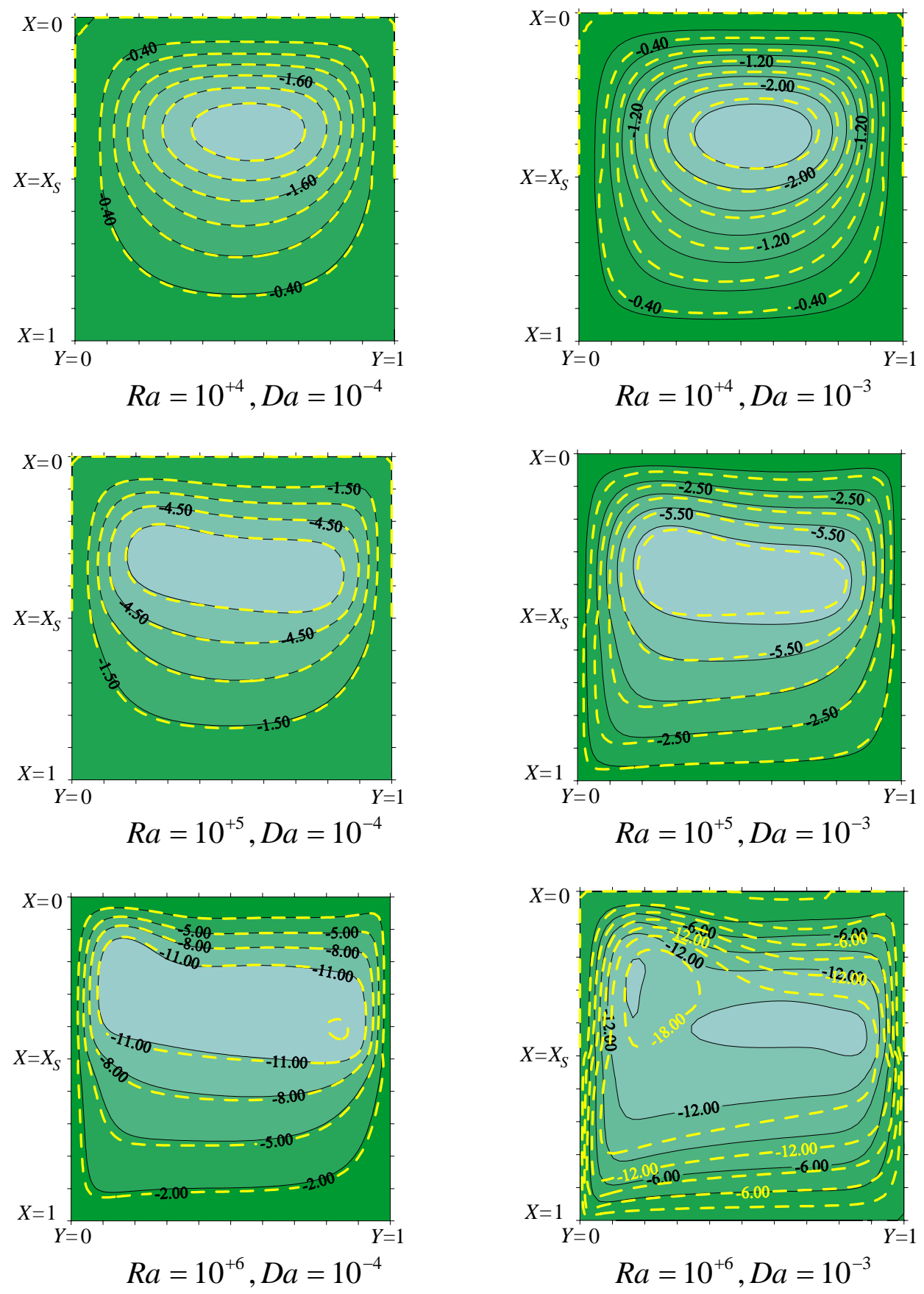

Figura 4.11.- Mapas de contorno para el potencial $\left(\Psi_{z}\right)$ modelos tridimensionales: - - B y J; Brinkman; Xs=0.5, $R c=1.0$ 
Conforme el $\mathrm{Da}$ se incrementa de $10^{-4}$ a $10^{-3}$ se puede observar como los efectos convectivos comienzan a ser más significativos sobre todo la contribución de transferencia de calor en el medio poroso, este fenómeno reflejado en la componente del vector potencial, indicando una alta actividad convectiva a altos valores de los números de Rayleigh y Darcy.

En cuanto a la comparación entre los modelos de Brinkman y las condiciones de Beavers y Joseph se puede observar que para valores bajos de $R a$ las líneas de corriente son iguales en ambas regiones, sin embargo conforme se aumenta el $\mathrm{Da}$ los efectos muestran diferencias, sobre todo en la región porosa, lo anterior por los efectos de la permeabilidad. El caso más drástico se tiene para $R a=10^{6}, D a=10^{-3}$ en la que los efectos de permeabilidad la región porosa y de términos de flotación en la región fluida son de gran importancia.

La comparación de los modelos en tres dimensiones se presenta en la Figura 4.12 en los planos $Y=0.25$ y $Y=0.5$ para la componente " $Z$ " del vector potencial, las condiciones extremas de $R a=10^{6}$ y $D a=10^{-3}$ para mostrar las comparaciones, en la figura se puede observar como no existen diferencias entre el mismo modelo para los diferentes planos, sin embargo la diferencia entre modelos es muy notoria sobre todo en las partes cercanas a la interregión.

En la Figura 4.13 se presenta la comparación de la temperatura para los modelos en dos y tres dimensiones mostrando isotermas a $Y=0.25$, en ella se observa que las diferencias se notan entre los modelos mientras que estas diferencias son mínimas para la misma formulación sin importar las dimensiones. Las diferencias más notorias se ubican cerca de la interregión, mientras que en las paredes los valores de temperatura son muy cercanos, lo anterior nuevamente hace notar que para valores altos de Rayleigh y Darcy las formulaciones muestran gran discrepancia en la cercanía de la interregión, esto indica que las discrepancias no son afectadas por resolver el modelo en tres dimensiones sino por la forma de acoplar las ecuaciones de transferencia de cantidad de movimiento, lo anterior sugiere un análisis más detallado en esta región, es en estas condiciones donde las condiciones de salto serían útiles para definir los efectos interregionales

La comparación en tres dimensiones para las condiciones de $R a$ y $D a$ antes mencionadas se muestran en la Figura 4.14, en ella se nota como a $Y=0$ los perfiles de temperatura muestran valores mayores para el modelo que considera la ecuación de Beavers y Joseph para el acoplamiento de la ecuación de cantidad de movimiento. 


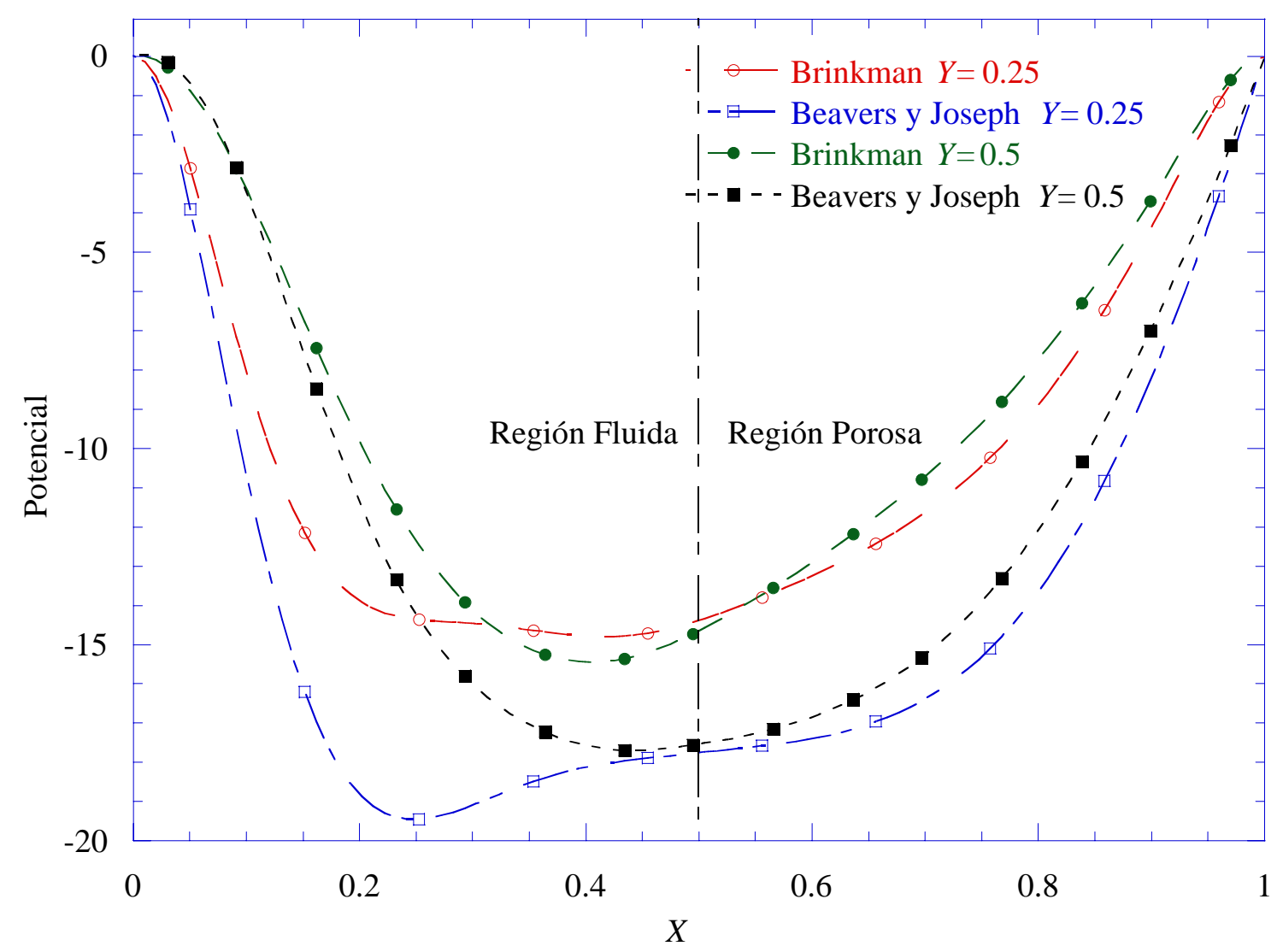

Figura 4.12.- Comparación de los perfiles del potencial $\left(\Psi_{z}\right) Y=0.25$ y 0.5, Modelos en 3D, $R a=10^{6}, D a=10^{-3}$ 


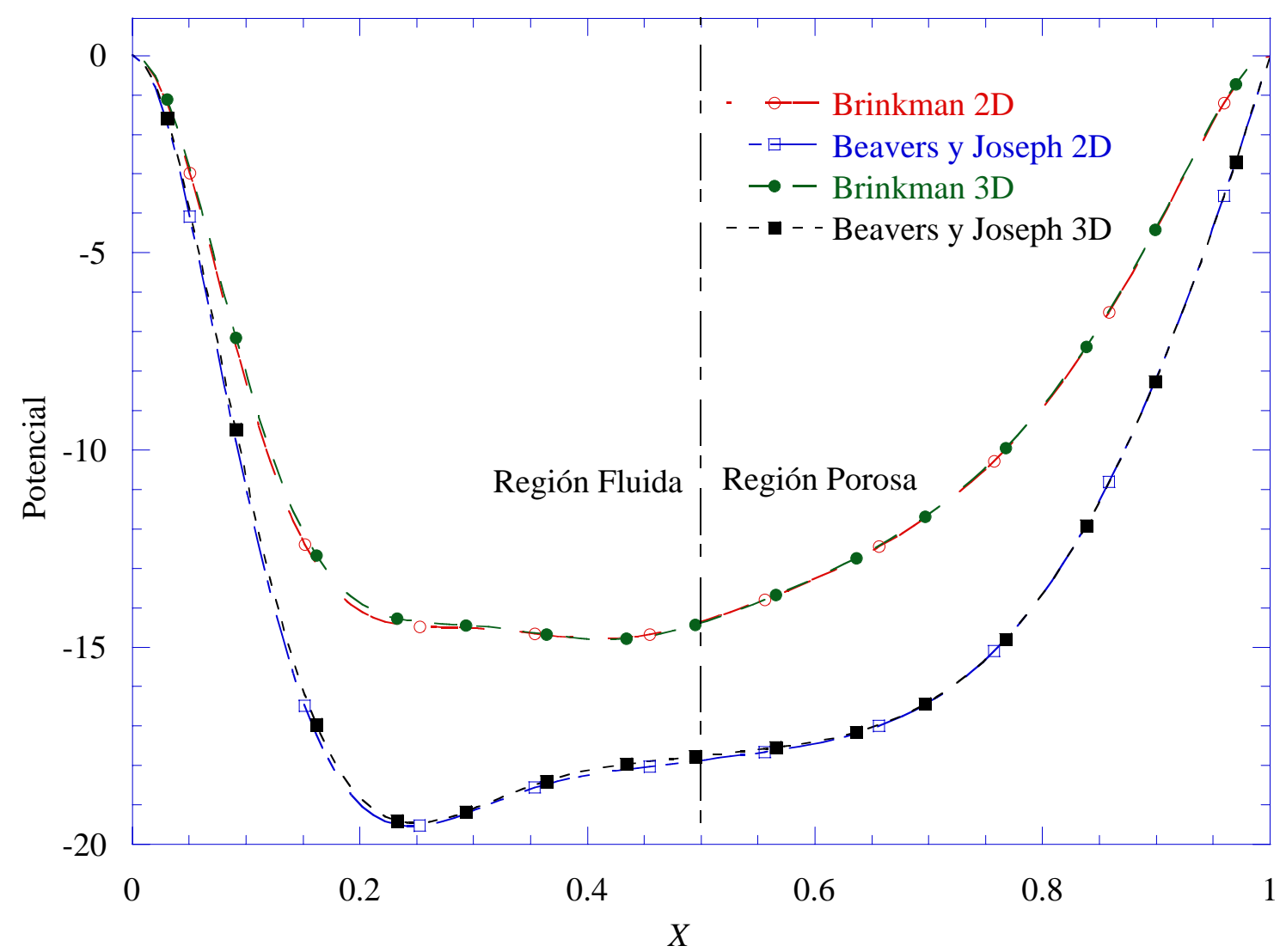

Figura 4.13.- Comparación de los perfiles del potencial $\left(\Psi_{z}\right) Y=0.25$, Modelos en 2 y 3D, Rayleigh $10^{6}$, Darcy $10^{-3}$ 


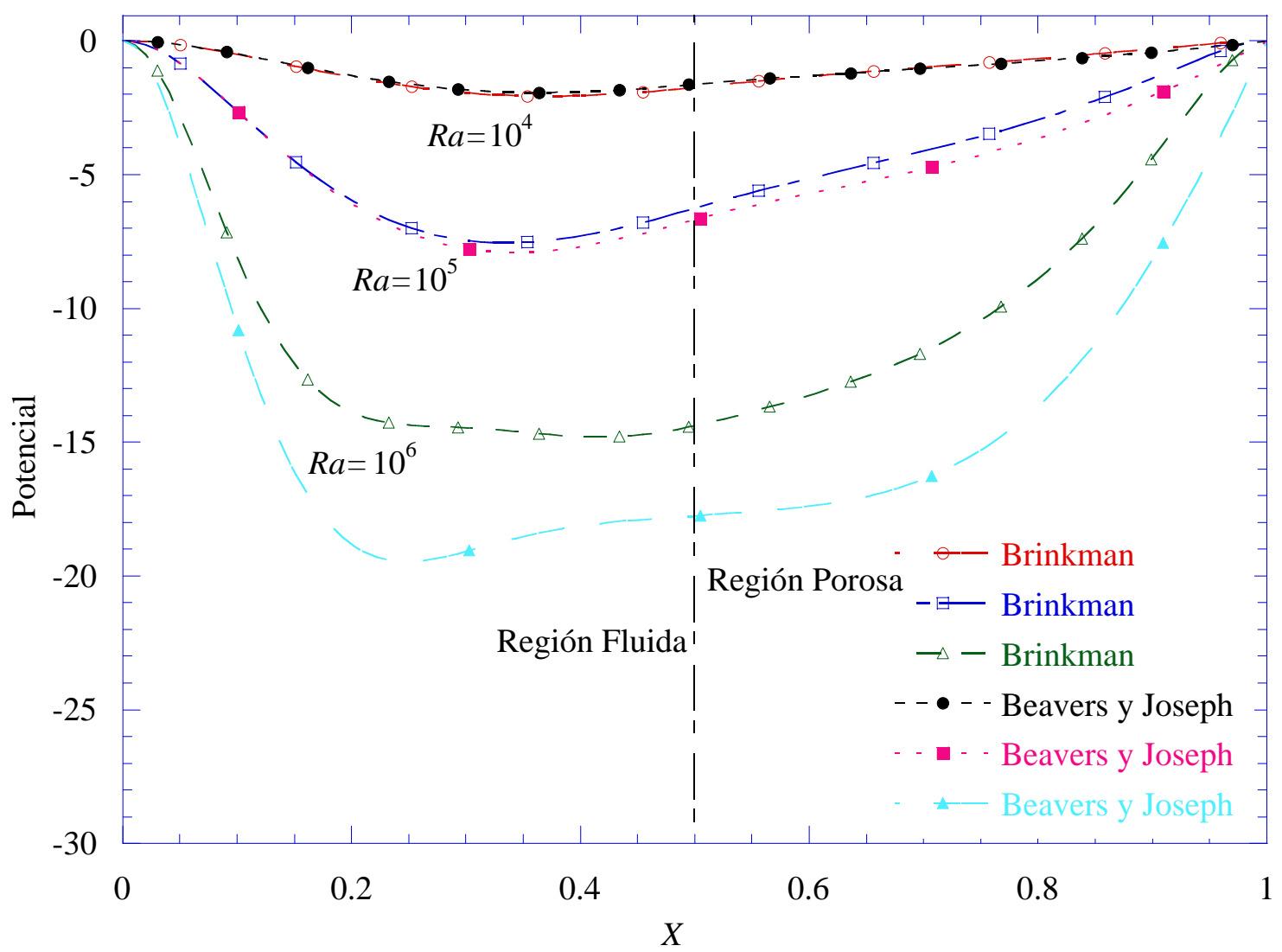

Figura 4.14.- Comparación de los perfiles del potencial $\left(\Psi_{z}\right) Y=0.25$, Modelos 3D, Darcy $=10^{-3}$ 

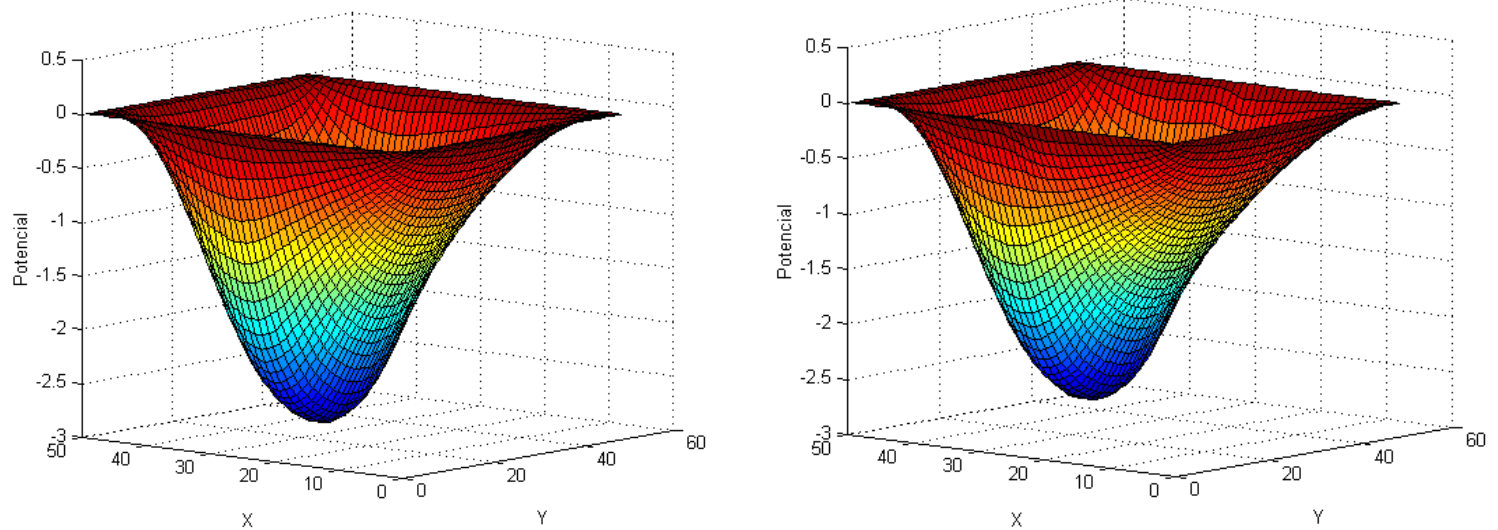

Rayleigh $10^{4}$, Darcy $10^{-3}$
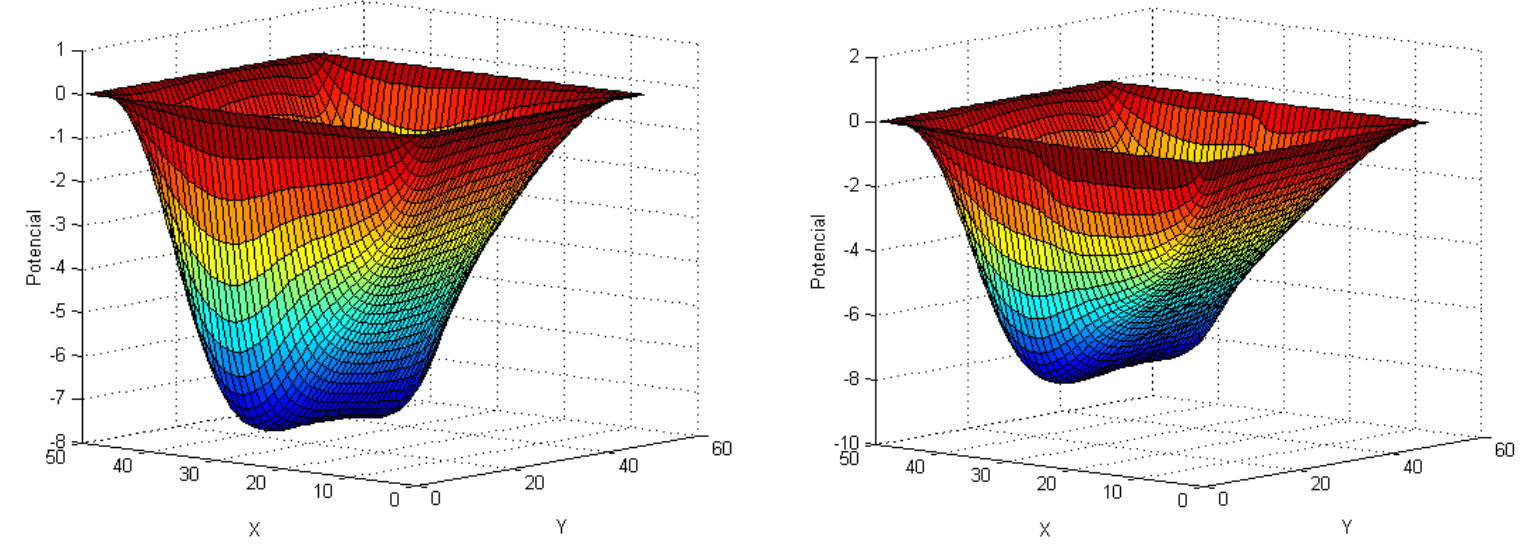

Rayleigh $10^{5}$, Darcy $10^{-3}$

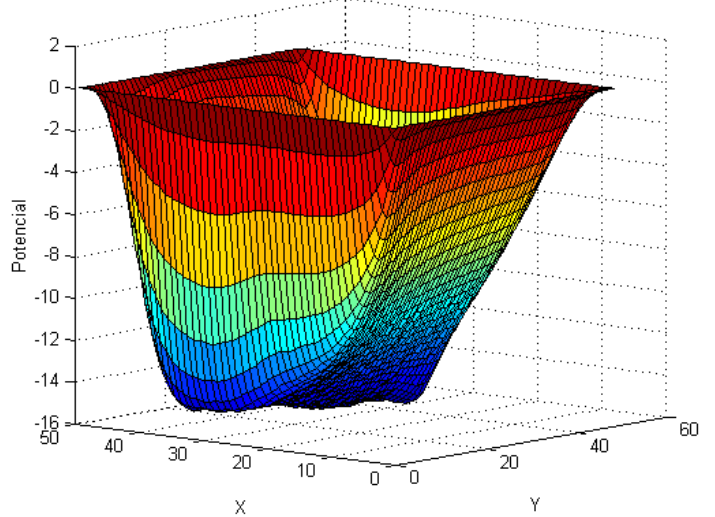

a)

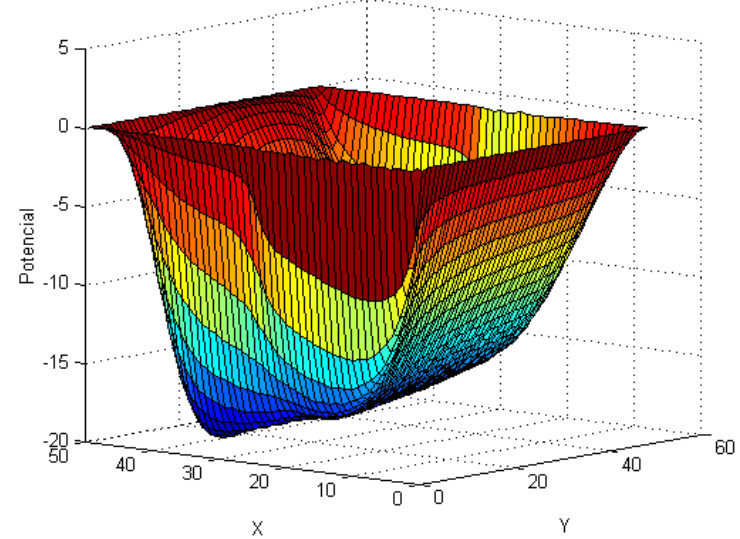

b)

Figura 4.15.- Superficies, para el potencial $\left(\Psi_{z}\right)$ modelos tridimensionales a) Brinkman b)Beavers y Joseph 
Para mostrar los efectos sobre el vector velocidad se evaluaron los componentes en cada dirección mediante la ecuación (2-38), la evaluación se lleva a cabo al final del proceso iterativo, hay que recordar que el vector velocidad no se considera como variable de iteración (ya que se sustituyó la formulación en términos de variables primitivas por la formulación vorticidad-vector potencial), de acuerdo a las condiciones de frontera utilizadas sólo la componente en la dirección “ $Z$ ” $\left(\Psi_{Z}\right)$ muestra variaciones importantes.

En la Figura 4.16 se muestran los mapas de contorno la componente " $Z$ ” del vector velocidad, en los mapas de contorno se muestran simultáneamente los resultados para el modelo que utiliza la corrección de Brinkman a la ecuación de Darcy (con líneas continuas) y el modelo que utiliza la condición de Beavers y Joseph (líneas segmentadas), de esta manera se puede realizar una comparación simultánea entre ambos modelos para la variación de dos números adimensionales.

Respecto a la componente " $Z$ " del vector velocidad se puede observar que la convección natural tiene su influencia conforme aumenta el Número de Rayleigh, esto es al pasar $10^{4}$ a $10^{6}$, lo anterior se hace evidente con la deformación de la célula (máximo) en la región fluida, llegando al grado de ubicarse el máximo en la parte superior izquierda para un $R a$ de $10^{6}$ y Da $10^{-3}$, una comparación de las figuras indican que los perfiles de velocidad son más densas en la región fluida cerca de las paredes caliente y fría a $R a=10^{5}$ y $10^{6}$ como una consecuencia del incremento de las fuerzas de flotación asociadas con el incremento del $R a, \mathrm{y}$ que en la región porosa se tiene una contribución conductiva a bajos $R a$.

Conforme el Da se incrementa de $10^{-04}$ a $10^{-03}$ se puede observar como los efectos convectivos comienzan a ser importantes en el medio poroso, este fenómeno reflejado en la componente del vector velocidad,, indicando una alta actividad convectiva a altos valores de los números de Rayleigh y Darcy. 

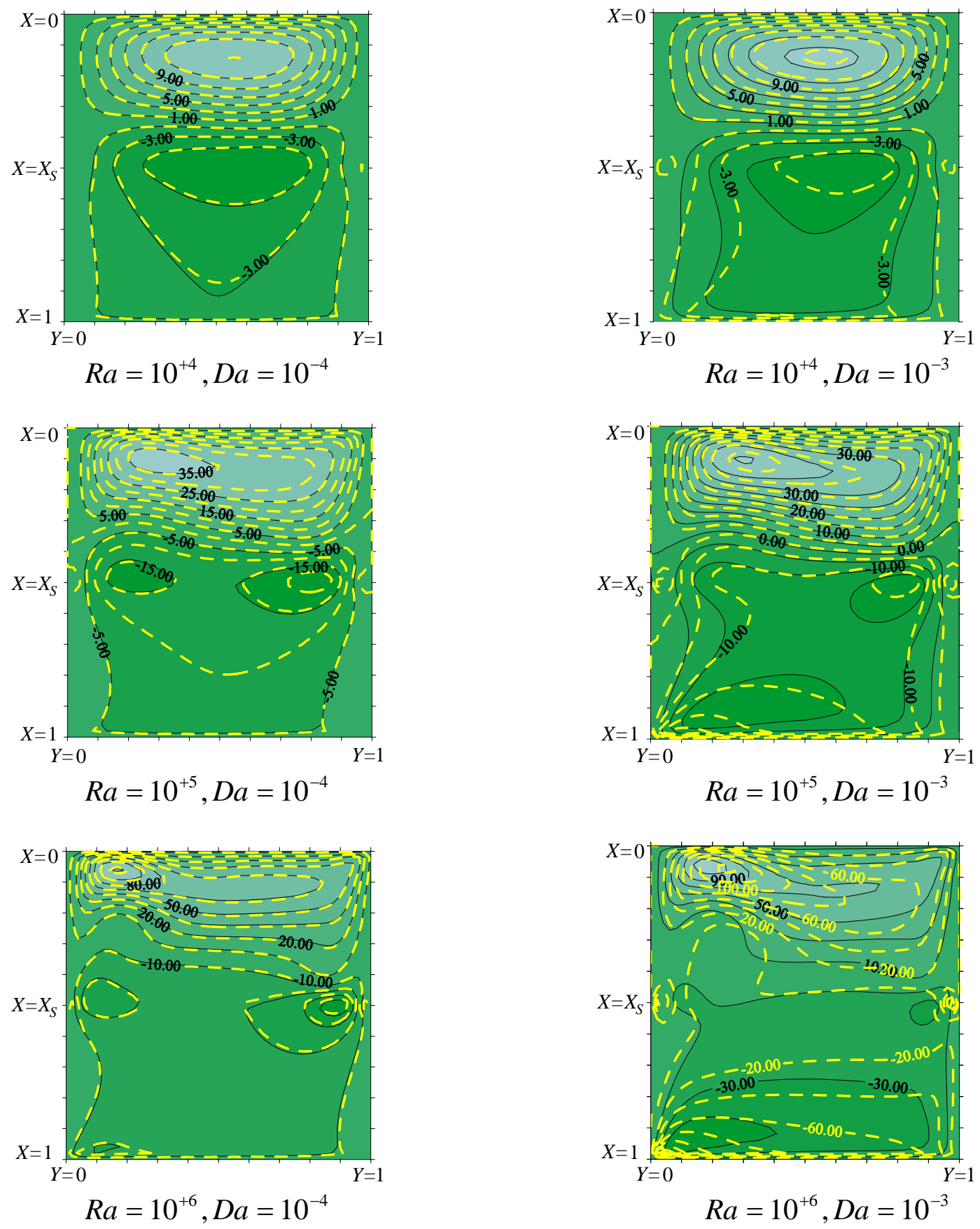

Figura 4.16.-Mapas de contorno para la componente $\mathrm{z}$ de la velocidad $(w)$ modelos tridimensionales: - - B y J; $\quad$ Brinkman, $X s=0.5, R c=1.0$ 
En cuanto a la comparación entre los modelos de Brinkman y las condiciones de Beaver y Joseph se puede observar que para valores bajos de $R a$ los perfiles para la componente de la velocidad son iguales en ambas regiones, sobre todo para el modelo que considera la corrección de Brinkman, sin embargo esta similitud es menor conforme se aumenta el $D a$, sobre todo en la región porosa, lo anterior por los efectos de la permeabilidad. Para caso de $R a=10^{6}, D a=10^{-3}$ en la que los efectos de permeabilidad la región porosa y de términos de flotación en la región fluida las diferencias son muy marcadas. Otro aspecto para considerar es la necesidad de utilizar una mejor interpolación en para la evaluación en los pontos interregionales sobre las paredes caliente y fría para el modelo que considera las condiciones de Beavers y Joseph (ver células en el mapa de contorno para $R a=10^{6}, D a=10^{-3}$ en la Figura 4.16)

En la Figura 4.17 se presenta la comparación para el perfil de la componente “Z” del vector velocidad para los modelos en dos y tres dimensiones mostrando los perfiles a $Y=0.25$ y 0.5 , en ella se observa que las diferencias se notan entre los modelos, mientras que estas diferencias son mínimas para la misma formulación. Las diferencias más notorias se ubican en la región fluida en la parte en donde la velocidad adquiere el máximo valor, lo anterior nuevamente hace notar que las discrepancias no son afectadas por resolver el modelo en tres dimensiones sino por la forma de acoplar las ecuaciones de transferencia de cantidad de movimiento.

La comparación de los modelos en tres dimensiones se presenta en la Figura 4.18 en los planos $Y=0.25$ y $Y=0.5$ para la componente " $Z$ " del vector velocidad, se tomaron las condiciones de $R a=10^{6}$ y $D a=10^{-3}$ para mostrar las comparaciones, en la figura se puede observar como las diferencias son notorias al comparar la formulación. 


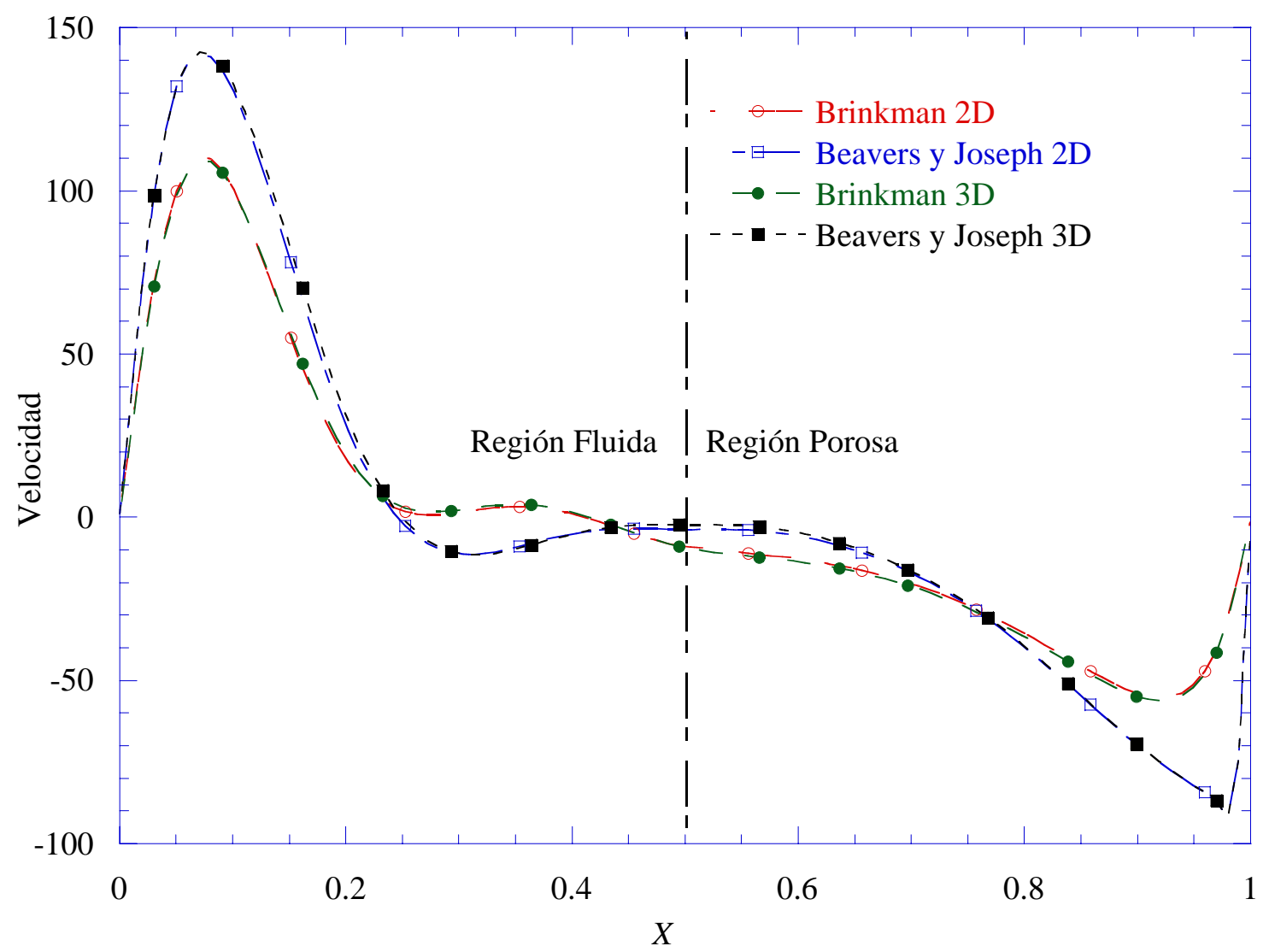

Figura 4.17.- Comparación de los perfiles de la componente $Z$ de la velocidad a $Y=0.25, Y=0.5$ Modelos en 2 y 3D, $R a=10^{6}$, $D a=10^{-3}$. 


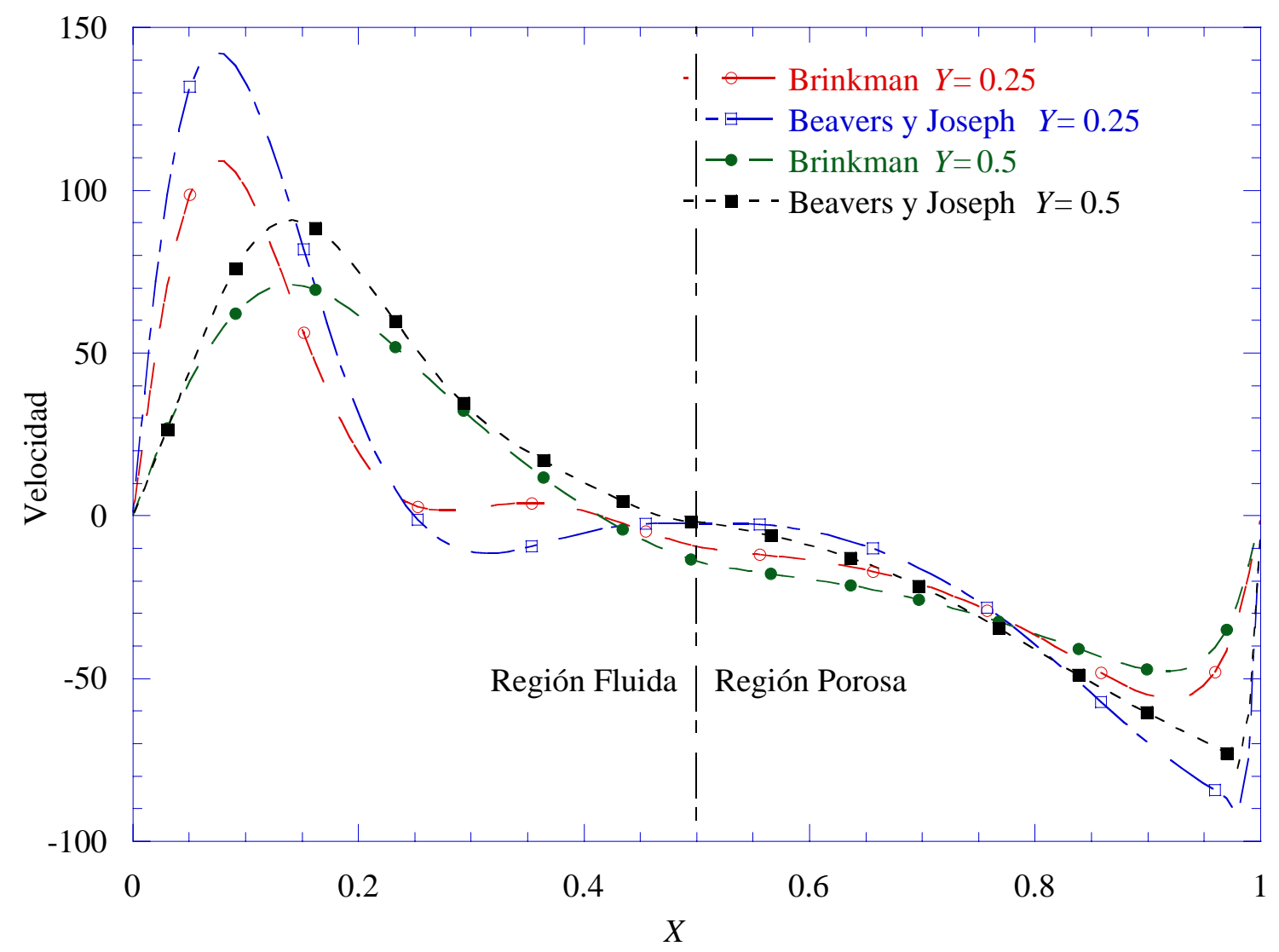

Figura 4.18.- Comparación de los perfiles de la componente $Z$ de la velocidad ( $w)$ a $Y=0.25$, $Y=0.5$; Modelos en 3D, $R a=10^{6}$, $D a=10^{-3}$. 
El efecto del $R a$ para ambos modelos se presenta en la Figura 4.19, los perfiles de velocidad se ubican a $Y=0.50$, en ella se observa que las diferencias entre modelos para un $D a$ constante son mínimas cuando se comparan diferentes $R a$, se esperaría que los efectos interregionales para la velocidad son importantes al incrementar el valor del $D a$ o bien la relación de las conductividades entre el fluido y el medio poroso, esto último se analizará en la siguiente sección.

La comparación en tres dimensiones para las condiciones de $R a$ y $D a$ antes mencionadas se muestran en la Figura 4.20, en ella se nota como a $Y=0$ para $R a=10^{6}, D a=10^{-3}$ los perfiles de velocidad muestran asimetría en ambos modelos con valores extremos en la formulación que utiliza las condiciones de Beavers y Joseph. 


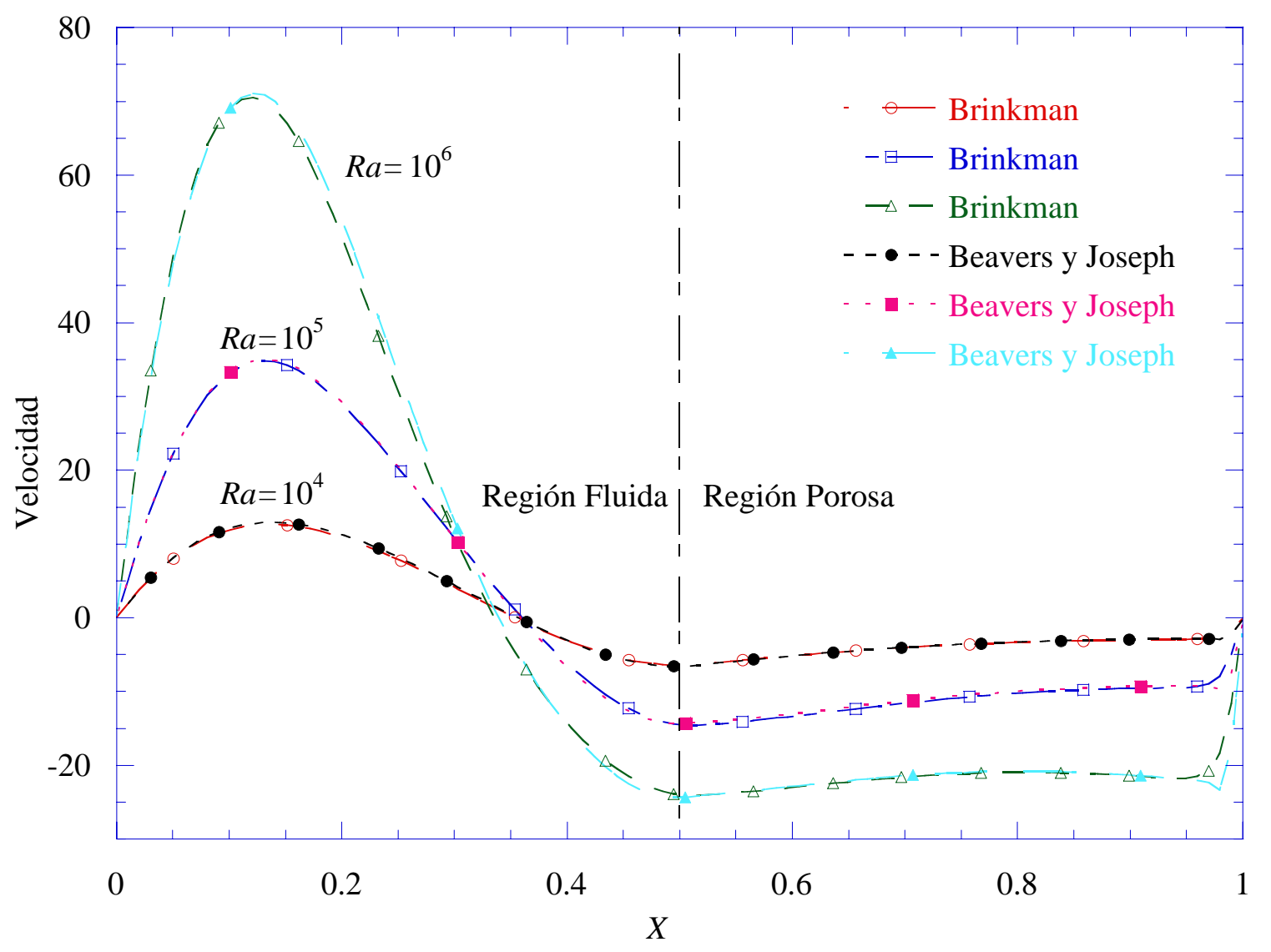

Figura 4.19.- Comparación de los perfiles de la componente $Z$ de la velocidad ( $w$ ) a $Y=0.5$, Modelos 3D, $D a=10^{-4}$. 

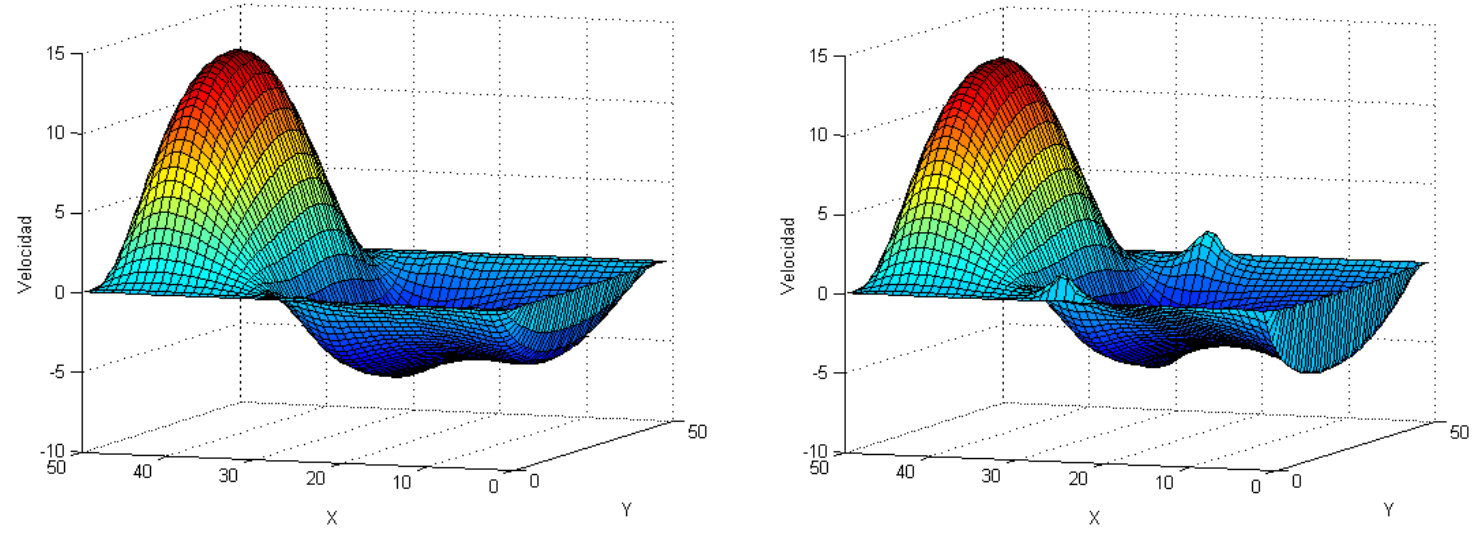

Rayleigh $10^{4}$, Darcy $10^{-3}$
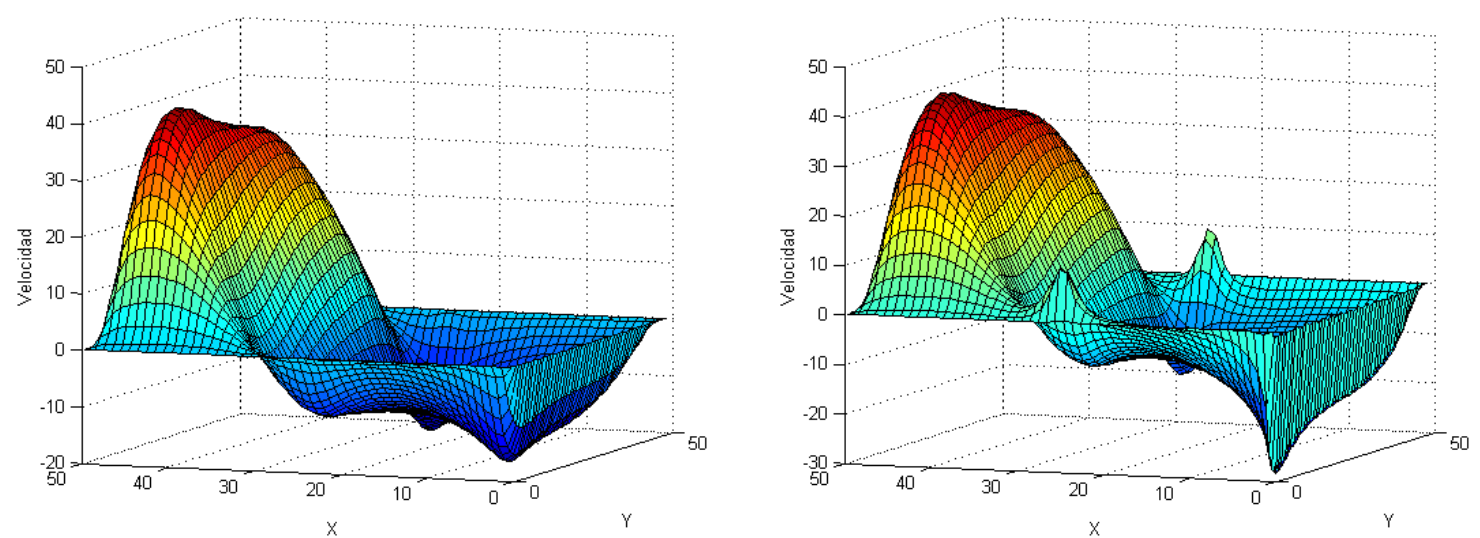

Rayleigh $10^{5}$, Darcy $10^{-3}$

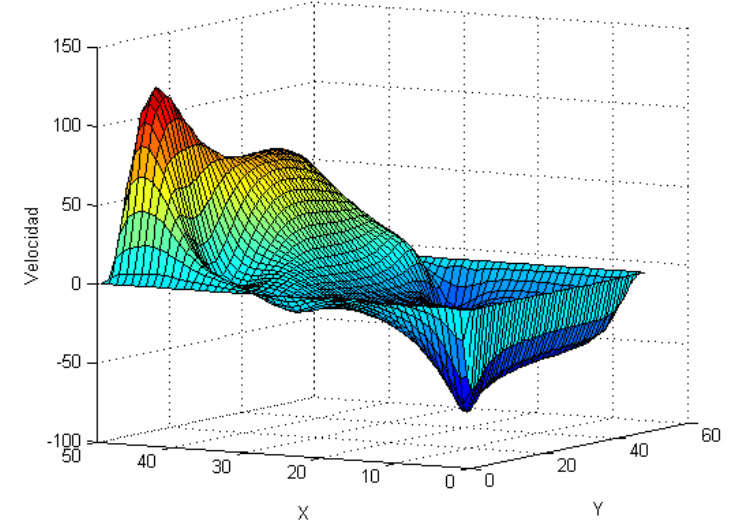

Rayleigh $10^{6}$ Darcy $10^{-3}$

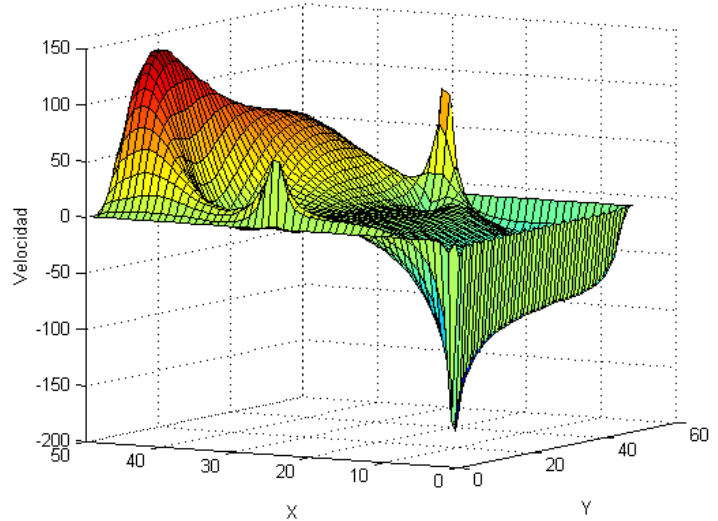

b)Beavers y Joseph

Figura 4.20.- Superficies para la componente $Z$ del vector velocidad (w), variación del $R a$ y $\mathrm{Da}$, modelos tridimensionales 


\subsection{Variación de la Relación de Conductividades.}

Autores como Le Peutrec y Lauriat (1990), han concluido que para fluidos de baja conductividad térmica se tienen pérdidas despreciables de calor y que su influencia comienza a ser considerable, en cuanto se incrementa el Número de Rayleigh. Todo lo anterior fue concluido para un sistema tridimensional y considerando una sola región, en la presente sección se muestran las gráficas realizadas para analizar el efecto en la relación de conductividades cuando se tienen dos regiones en una cavidad tridimensional; los valores utilizados para la variación de la relación de conductividades $(R c)$ son: 1, 5 y 10.

En la figura 4.21 se muestran los mapas de contorno para las isotermas, comparando la formulación que consideran la correcciones de Brinkman a la ecuación de Darcy con la que utiliza las ecuaciones de Beavers y Joseph, los mapas de contorno se muestran para un $\mathrm{Da}$ fijo y haciendo variar el $R a$ desde $10^{4}$ a $10^{6}$ y una relación de conductividades que van desde 1 hasta 10, Incrementando el valor de la relación de conductividades resulta en un claro dominio de la transferencia conductiva de calor en la región porosa, mientras que la convección continua dominando en la región fluida. La diferencia entre los tipos de transferencia de calor son notorios inclusive a bajos $R a$, el efecto que tiene un valor grande de conductividad en la región porosa afecta los perfiles de temperatura en la región fluida, así pues para el mapa de contorno a $R a=10^{6}$ y $R c=10$ se observa como no existe una diferencia importante entre modelos en la región porosa, sin embargo en la región fluida se tienen isotermas que comienzan a alejarse a partir de la interregión, nuevamente surge la necesidad de utilizar condiciones interregionales que garanticen el adecuado acoplamiento entre las ecuaciones en el fluido y medio poroso, en este aspecto los resultados reportados por Ochoa y Whitaker (1997; 1998 a,b) serán de gran utilidad. 

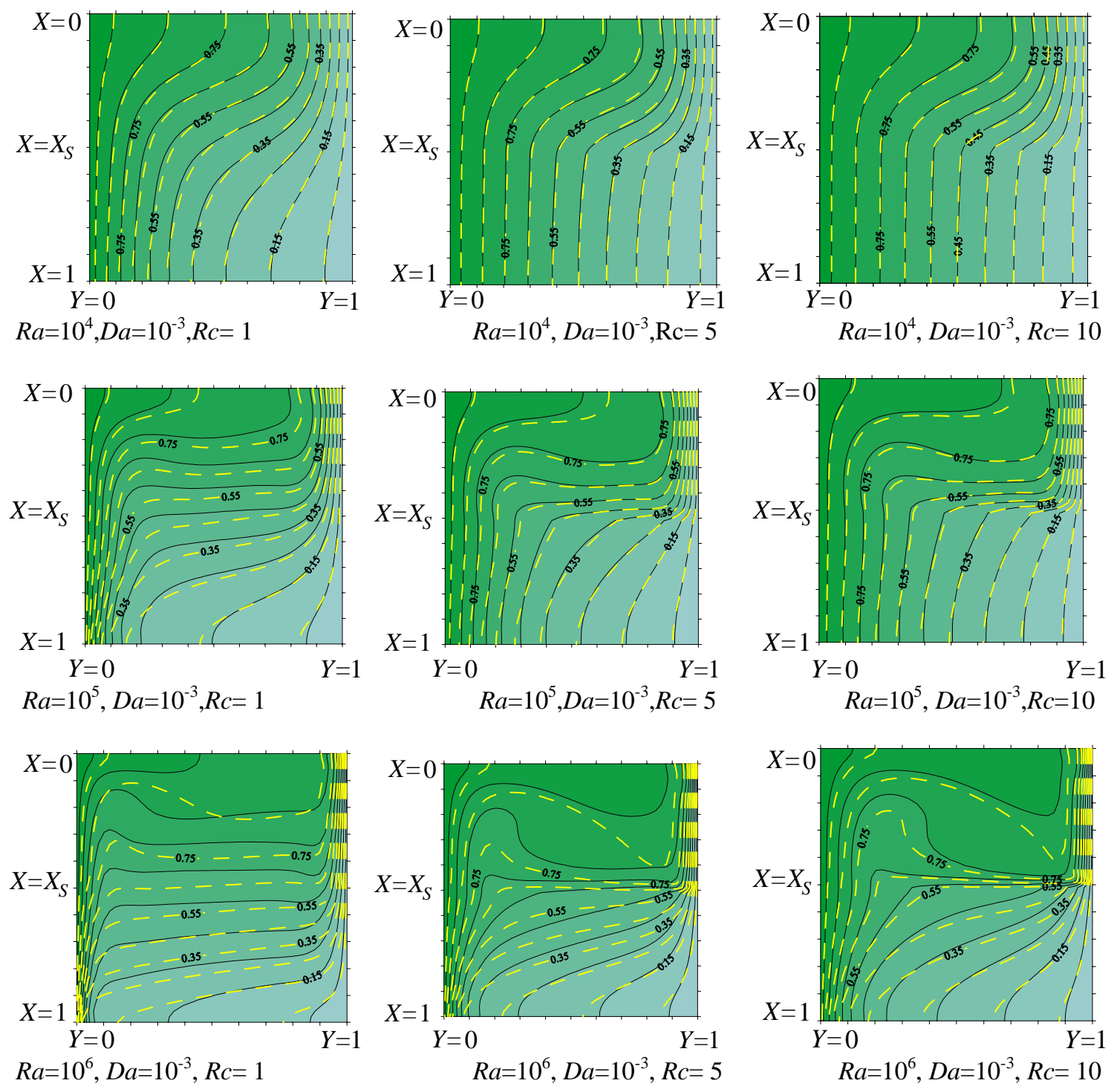

Figura 4.21- Isotermas para analizar la relación de conductividades, modelos tridimensionales: - B y J.; Brinkman. 


\subsection{Variación de la interregión.}

Como se ha mencionado en las pruebas anteriores los efectos convectivos se presentan en la región fluida mientras que en el medio poroso a bajos $R a$ el fenómeno predominante es el conductivo, el objetivo de variar la interregión es analizar la influencia de los efectos conductivos sobre la región fluida, con un $R a$ constante se comienza con una cavidad llena de medio poroso hasta terminar con una cavidad vacía (ver Figura 4.22)

En la Figura 4.23 se ilustra el efecto de la posición de la interfase fluido-medio poroso sobre las isotermas, velocidad, líneas de corriente y Número de Nusselt. Cuando se tiene únicamente medio poroso en la cavidad, se forma flujo unicelular (ver líneas de corriente) el cual empieza a ubicarse en la región fluida conforme se presenta la región fluida. La magnitud de las líneas de corriente va disminuyendo a medida que aumenta la posición de la interregión, lo que es consecuencia de la resistencia de flujo mucho más lento que se forma cerca de la frontera en $Y=0$.

El efecto sobre las isotermas no es considerable ya que se utilizó una relación de conductividades $=1$, de igual forma los perfiles para la componente " $Z$ " de la velocidad no muestran gran diferencia entre modelos.

Como consecuencia de agregar un término de segundo orden a la ecuación de transferencia de cantidad de movimiento en el modelo de Brinkman, los mapas de contorno para la componente $Z$ del vector potencial muestran diferencias significantes en para la cavidad completamente llena de medio poroso, este mismo efecto se observa para los mapas de contorno de la velocidad.

Para realizar pruebas al modelo numérico, se comparó con datos proporcionados por el Dr. Jiménez-Islas para la cavidad cúbica llena de medio poroso en donde se usó la ley de Darcy con la extensión de Brinkman. Esta cavidad está calentada diferencialmente como se muestra en la Figura 4.1 y en ambos casos se nutilizó el criterio potencial-vorticidad, en cuanto al método numérico el Dr. Jiménez resolvió el sistema de ecuaciones diferenciales mediante el método Runge-Kutta-Fehlberg con control adaptivo de etapa. El problema lo considera un $\operatorname{Pr}=0.71, R a=10^{6}$ y $D a=10^{-4}$ con $15 \times 1 \times 15$ puntos de diferencias finitas en malleo constante. En la Figura 4.24 se muestra la compración de ambos modelos observándose diferencias insignificativas resultado del método numérico y tipo de malla utilizada. 

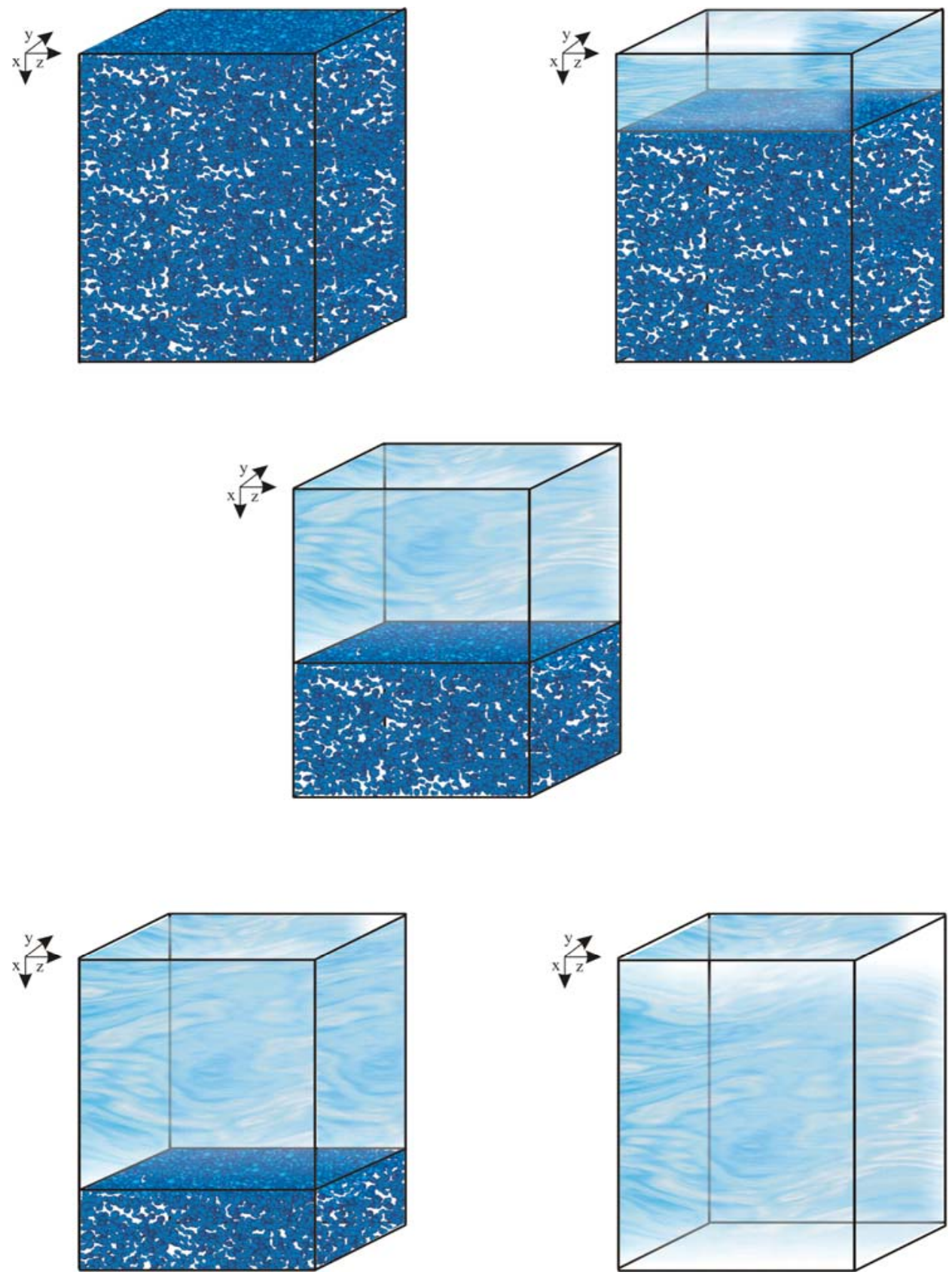

Figura 4.22- Posición de la interregión para las pruebas en tres dimensiones. 

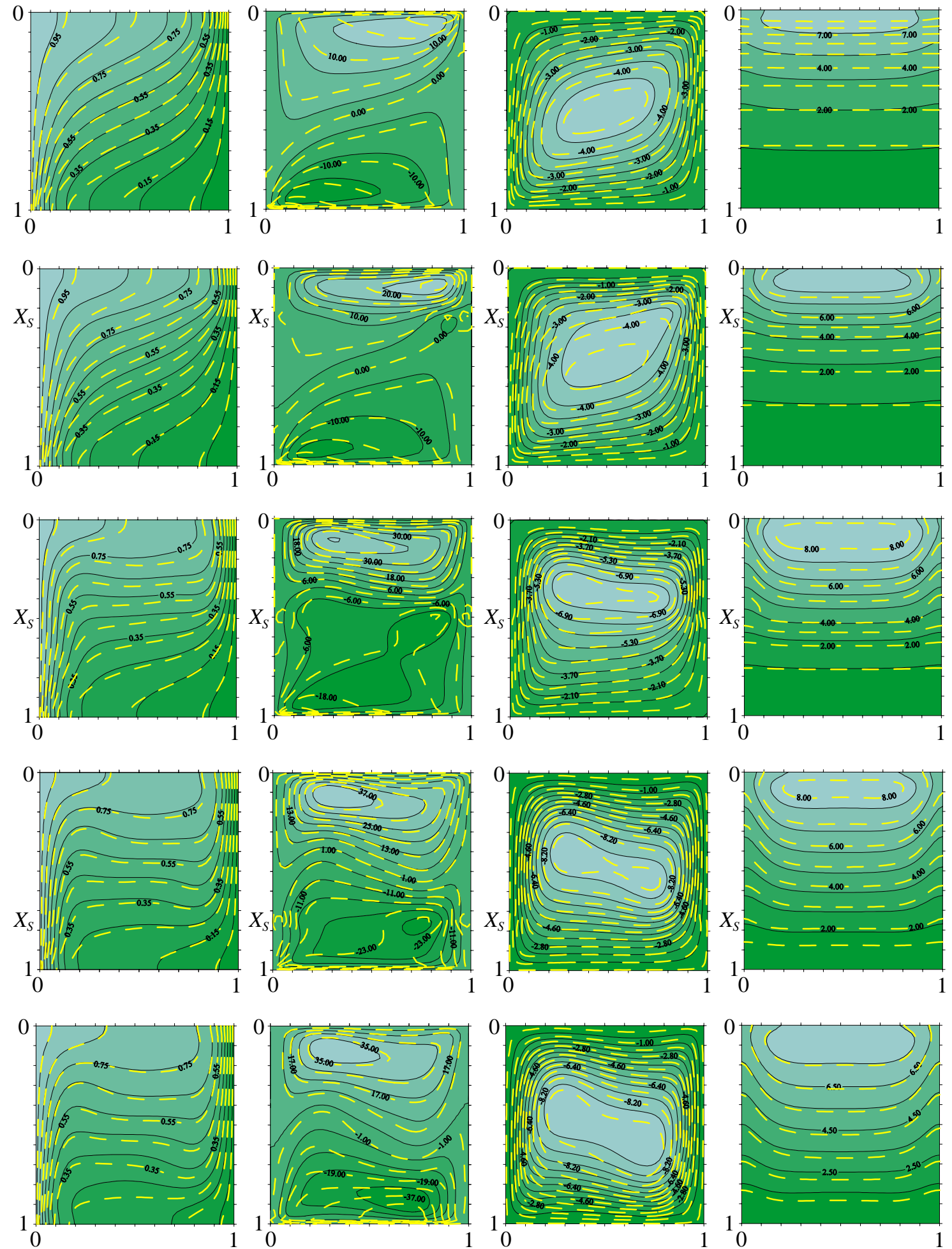

b)

c)

d)

Figura 4.23- Variación de la interregión: a)Isotermas b)Componente $Z$ del vector velocidad c)líneas de corriente d)Número de Nusselt, modelos tridimensionales: - - B y J; Brinkman, $R c=1.0$ 

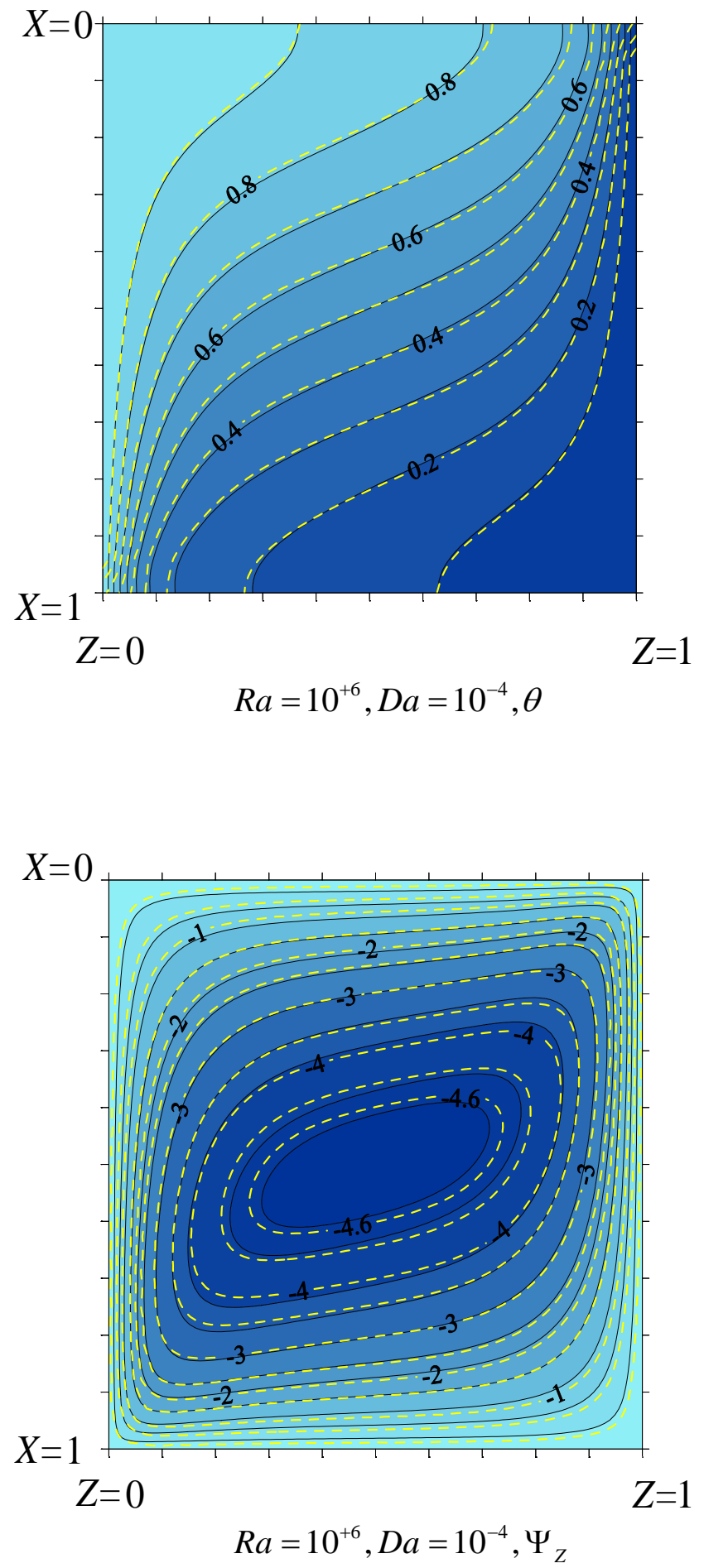

Figura 4.24.-Mapas de contorno para la componente $\mathrm{z}$ de la velocidad $(w)$ modelos tridimensionales: - - Jiménez-Islas, Brinkman, Xs=0.5, $R c=1.0$ 


\subsection{Efecto del Término convectivo}

Todos los resultados mostrados previamente no toman en consideración los efectos inerciales en el medio poroso, en la Figura 4.24 se comparan los mapas de contorno de temperatura y la componente $Z$ del vector potencial, a $Y=0.5$ incluyendo los efectos inerciales en ambos modelos tridimensionales, la comparación muestra que las predicciones utilizando la corrección de Brinkman es menos afectada por la inclusión de los efectos inerciales. Los resultados sugieren que los efectos que la adición del término convectivo en la ecuación de momentum para la región porosa puede tener efectos importantes en la transferencia de energía, por lo que se necesitaría realizar un estudio sistemático de los efectos convectivos en la región porosa, sin embargo, de acuerdo con desarrollos realizados por Whitaker (1996) y Ochoa-Tapia y Whitaker (1998a), demuestran que previamente deben de agregarse otros términos a la condición interregional.

Varios autores ( Lauriat y Prasad 1987; Jiménez-Islas y col. 1999) han concluido que los efectos viscosos en el medio poroso son importantes a partir de valores del número de Darcy de $10^{-4}$, en las figuras 4.26 y 4.27 se comprueba lo anterior, aunque en la Figura 4.26 no es muy notoria la conclusión anterior, en la Figura 4.27 se muestran los perfiles para la velocidad, en ellas se observa como a valores de $10^{-2}$ y $10^{-3}$ existen cambios importantes entre modelos lo anterior es insignificante a partir de valores de $D a=10^{-4}$ 

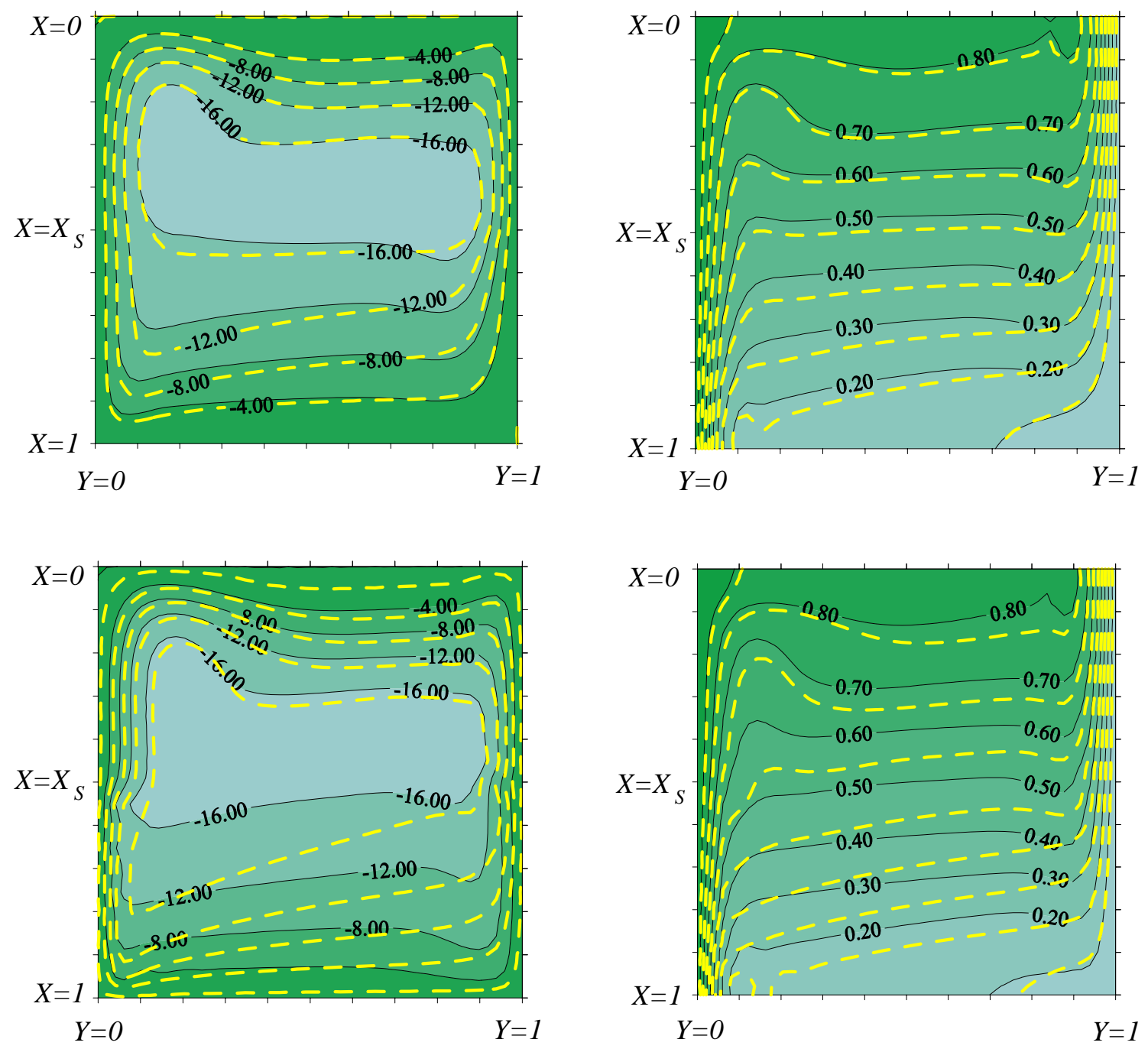

Figura 4.25.- Término convectivo: mapas de contorno para la componente $\mathrm{Z}$ de la velocidad (w) modelos tridimensionales: - - B y J; Brinkman. 

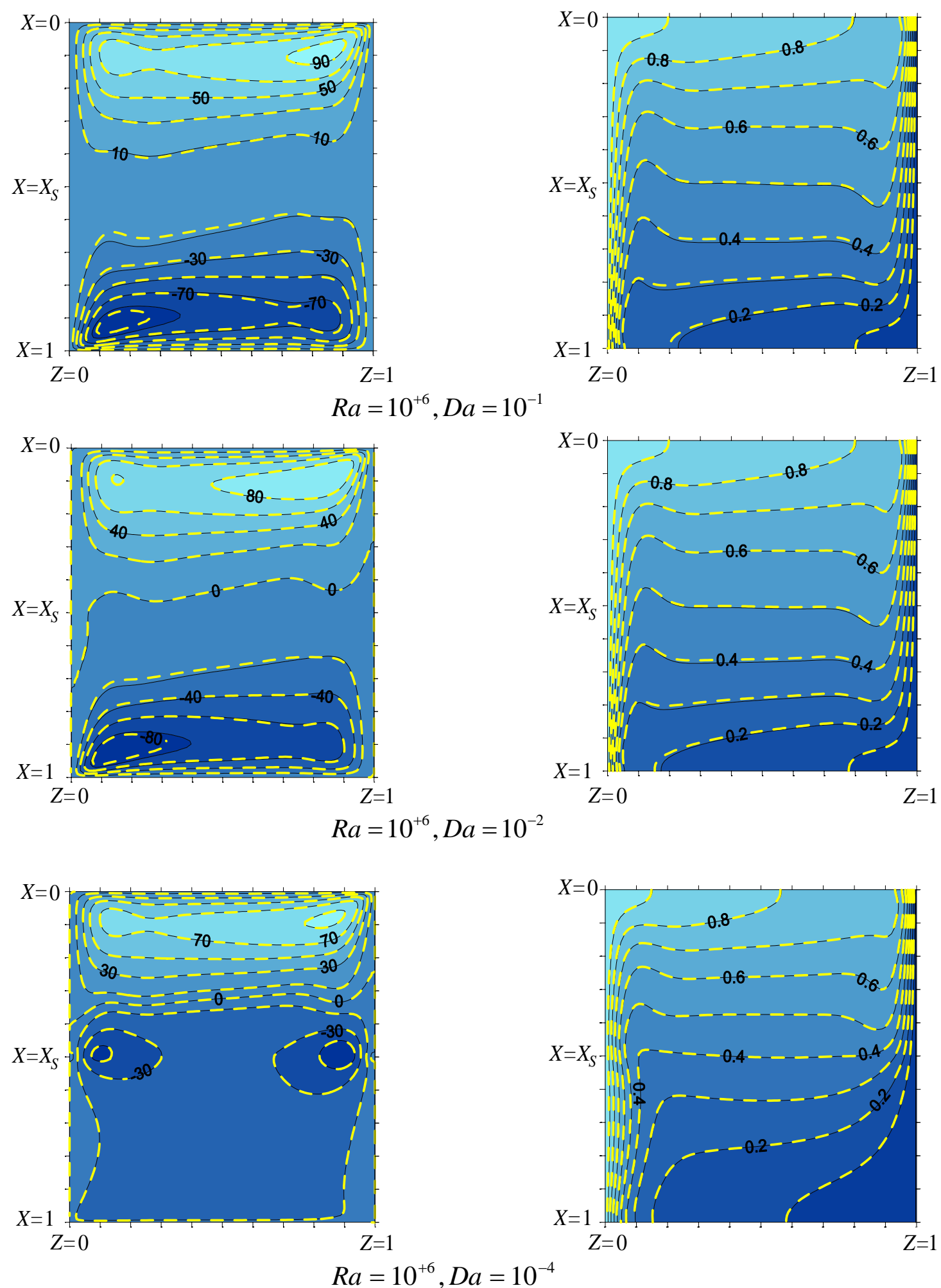

Figura 4.26.- Mapas de contorno para la componente $\mathrm{Z}$ de la velocidad (w) e isotermas Con término convectivo : - - Sin término convectivo: Modelo de Brinkman. 

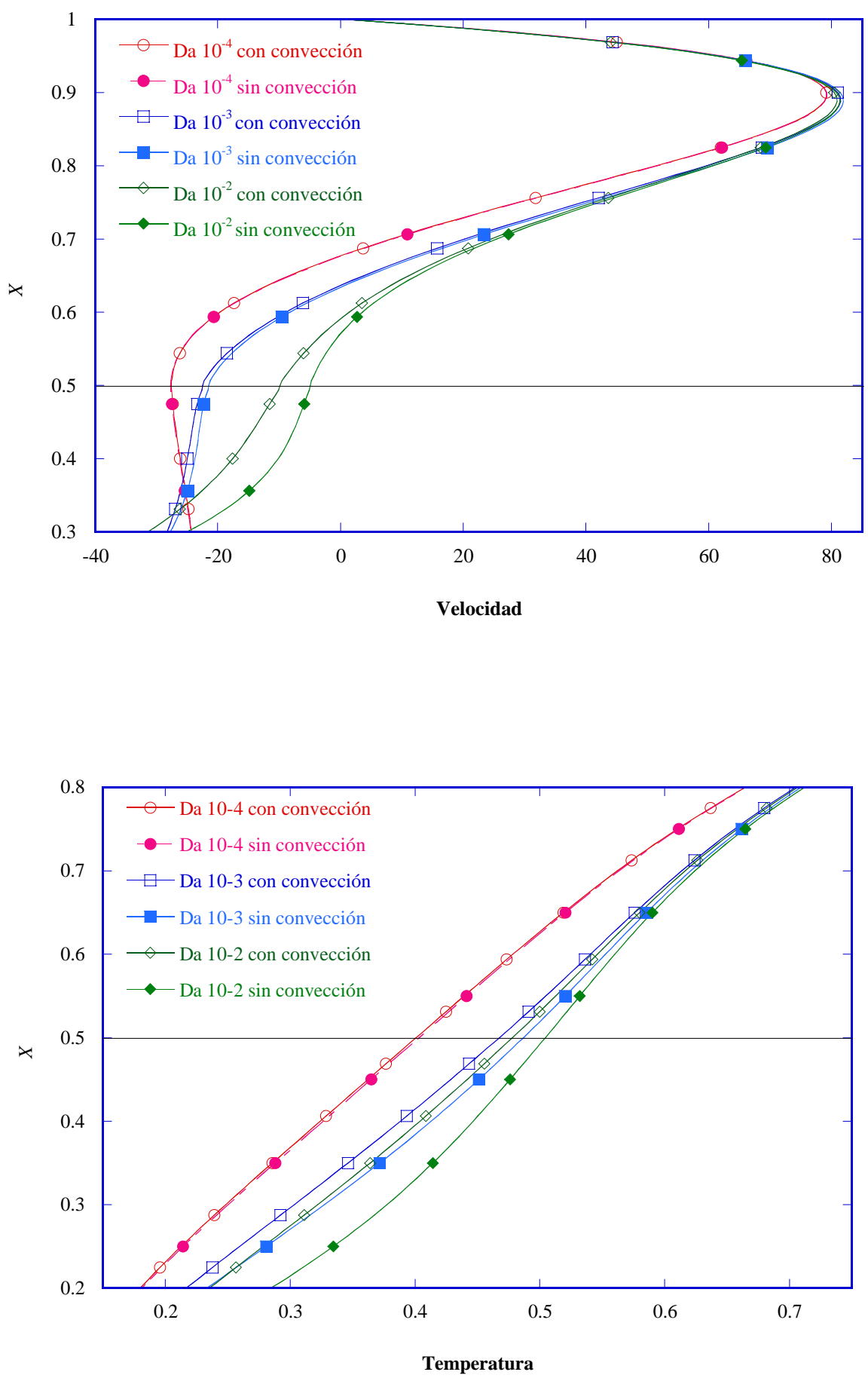

Figura 4.27.- Influencia del término convectivo: Componente $\mathrm{Z}$ de la velocidad $(w)$. 


\subsection{Pruebas considerando efectos tridimensionales.}

En la mayor parte de las pruebas se utilizan las condiciones de frontera especificadas en la Figura 4.1, ya sea en su forma tridimensional o en la simplificación para el modelo en dos dimensiones, lo anterior para analizar los efectos de las fuerzas de flotación. La siguiente prueba tiene como objetivo analizar los efectos tridimensionales en una cavidad completamente llena de medio poroso con las condiciones de frontera especificadas en la Figura 4.28, los resultados se comparan con los reportados por Jiménez (1999) para ello se utilizan números de Rayleigh de medio poroso, $R a_{p}=R a D a$ de 10 y 1000 , con una malla de $25^{3}$ nodos.

En la Figura 4.29 se muestran los mapas de contorno para las isotermas, y las componentes $X$ y $Z$ del vector potencial, ambos modelos muestran resultados similares con algunas diferencias en la prueba de Rap=1000, aún más en las isotermas ya que a este valor del Rayleigh las fuerzas de flotación son muy importantes, las diferencias se hacen notar an la parte superior de la gráfica y se le atribuyen a cuestiones numéricas, sobre todo por la utilización de espaciamiento constante. 


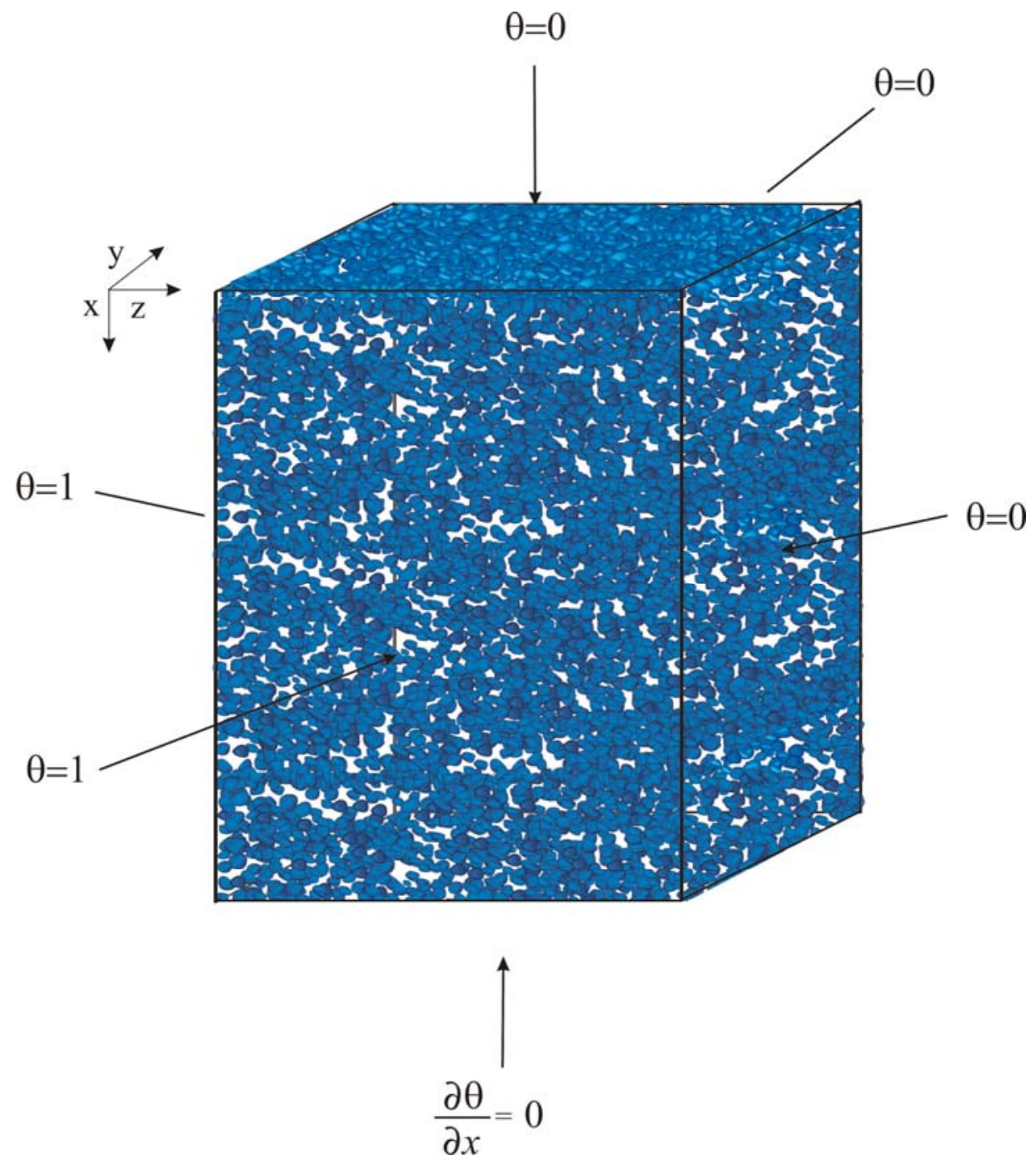

Figura 4.28 Condiciones de frontera para analizar efectos tridimensionales. 

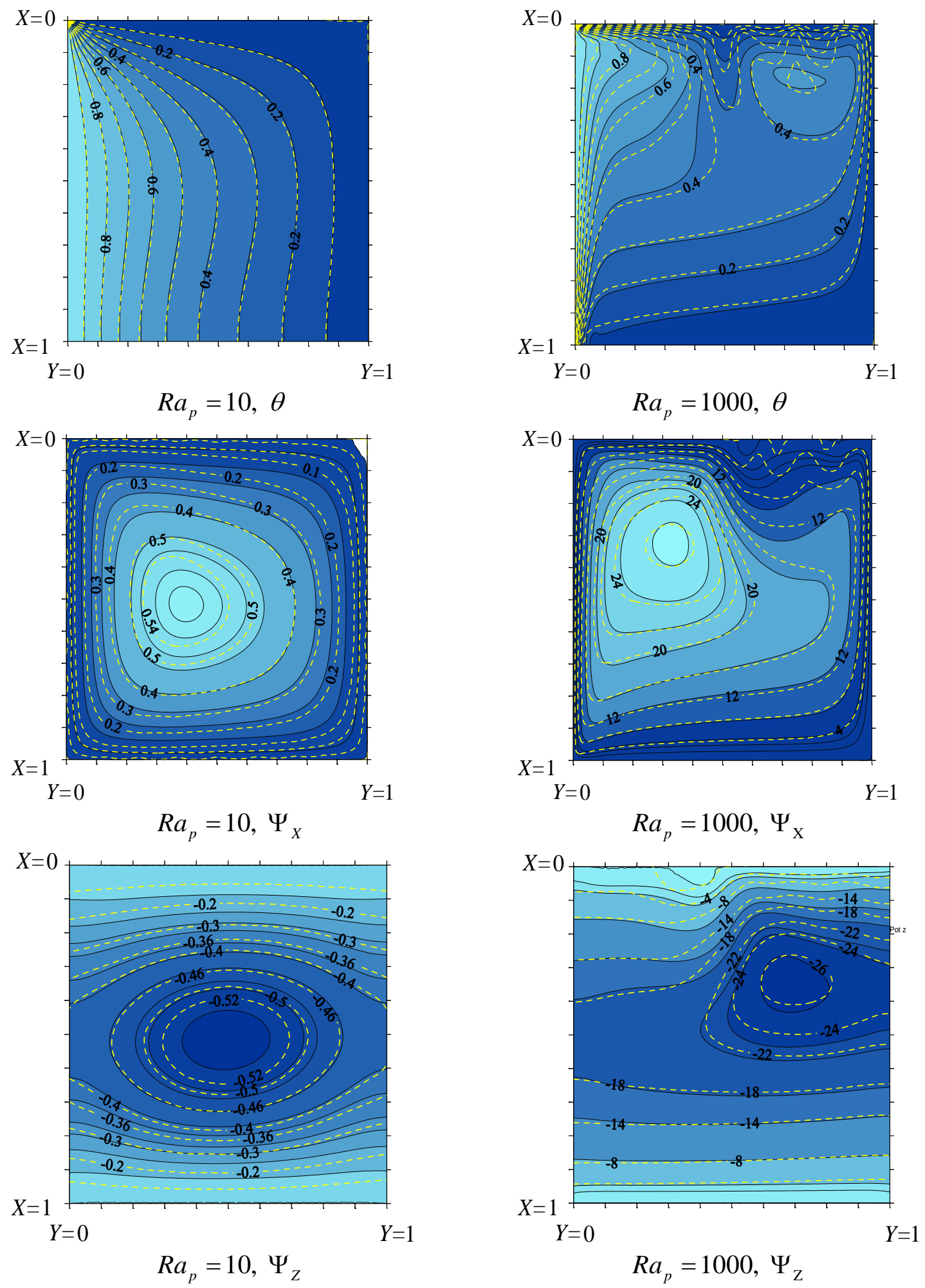

Figura 4.29.- Mapas de contorno para la temperatura y las componente $X$ y $Y$ del potencial: Presente trabajo (Brinkman), -- Jiménez (1999). 


\subsection{Pruebas al modelo de difusión doble}

\subsubsection{Variación de la interregión}

En pruebas anteriores se ha demostrado que los efectos convectivos adquieren importancia en la región fluida y se ven influenciados en gran medida por la forma de acoplar las ecuaciones en ambas regiones, para analizar los efectos del término de flotación (combinado) con efectos térmicos y másicos, se presenta la siguiente prueba que consiste en incrementar la posición de la interregión fluido-medio poroso, la variación del espesor se lleva a cabo considerando primeramente una cavidad completamente llena por el medio poroso, con paredes horizontales a diferentes temperatura y concentración, la segunda serie de resultado se obtiene con una cavidad compuesta por tres cuartas partes de medio poroso y una cuarta parte de fluido en la parte superior, y así sucesivamente hasta tener la cavidad con fluido, como se muestra en la Figura 4.30.

En la Figura 4.31 se muestra el efecto de la posición de la interfase fluido-medio poroso sobre las isotermas, líneas de concentración, líneas de corriente y velocidad, la prueba se llevó a cabo para un valor $R a=10^{4}, D a=10^{-4}$, en la figura puede observar que cuando se tiene únicamente medio poroso en la cavidad, se forma flujo unicelular centrado (ver líneas de corriente) el cual empieza a subir conforme se presenta la región fluida. La magnitud de las líneas de corriente va disminuyendo a medida que aumenta la posición de la interregión, lo que es consecuencia de la resistencia de flujo mucho más lento que se forma cerca de la frontera en $Y=0$. La figura muestra también como manteniendo todos los parámetros constantes los perfiles de temperatura y principalmente los perfiles de concentración tienen cambios considerables, alcanzando los valores máximos cuando se tiene una cavidad llena de fluido, los perfiles para esta situación coinciden con los presentados por Sezai y Mohamad (1999; 2000) 

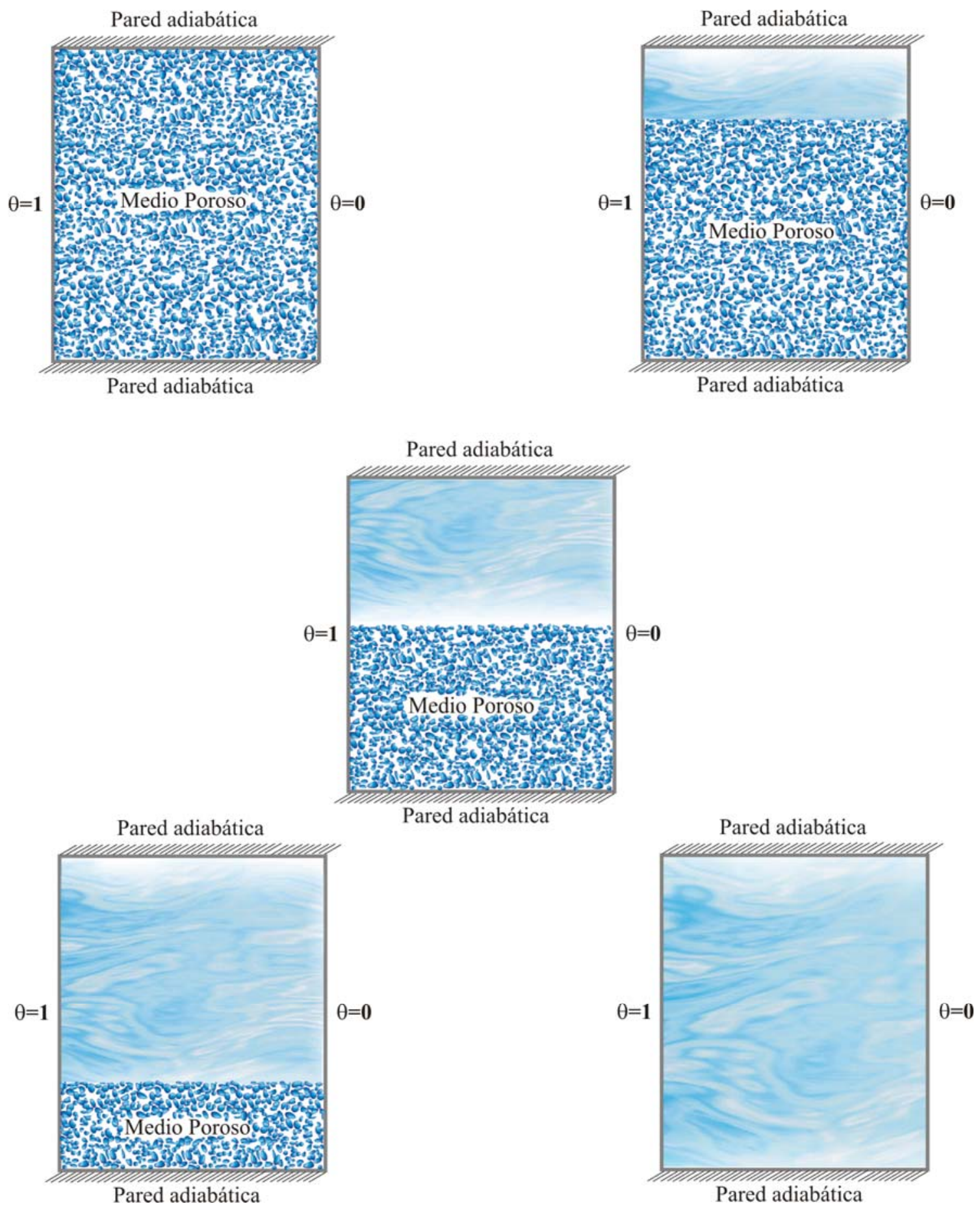

Figura 4.30.- Condición de frontera para la variación de la interregión, modelo bidimensional. 

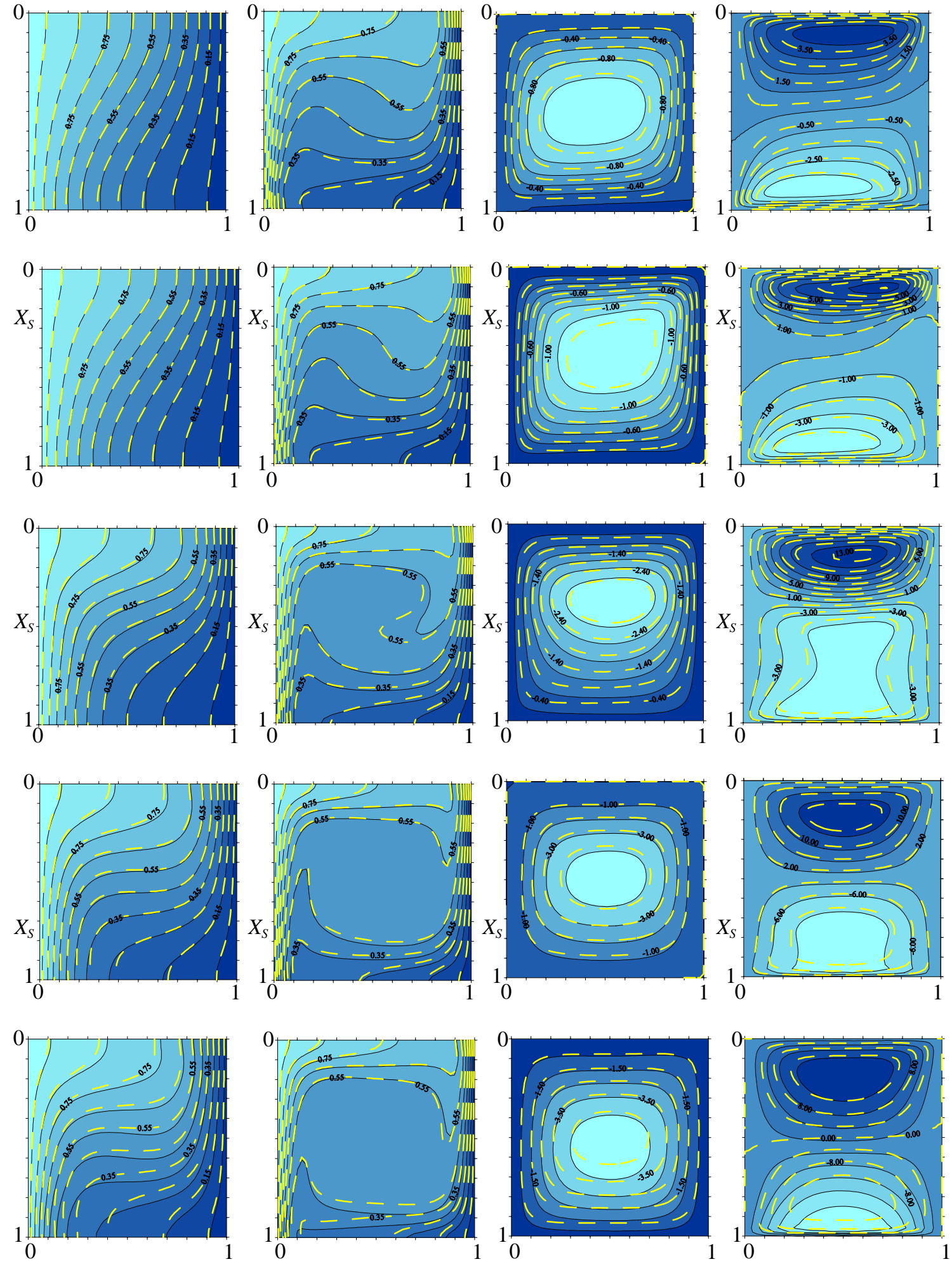

Figura 4.31.- Mapas de contorno para la prueba de la variación de la interregión: a)Isotermas b)líneas de concentración c)líneas de corriente d) velocidad. 


\subsubsection{Variación del Número de Flotación.}

El flujo convectivo considerado en difusión doble puede ser dividido en dos tipos:

a) Fuerzas de ocasionado por la transferencia de calor, por ejemplo flujos dominados por los efectos de flotación debido a los cambios de temperatura, y

b) flujo por transferencia de masa, donde los efectos de flotación son dominados por cambios de la densidad asociados con variaciones de concentración.

En el primer caso el número de flotación es igual a cero (ver ecuación 2.48), esto significa que los campos de temperatura están acoplados a los campos de flujo y no a los campos de concentración, sin embargo, el campo de concentración depende de los campos de temperatura y de flujo, la diferencia entre los campos de temperatura y concentración están indicadas por el número de Lewis (ver ecuación 2.17), en otras palabras, los campos de temperatura y concentración son idénticos sólo en el caso en que el Lewis sea igual a la unidad.

Para llevar a cabo la prueba se considera una cavidad completamente llena de medio poroso con las condiciones de frontera mostradas en la Figura 4.32 y haciendo variar el número de flotación mediante la modificación del número de Rayleigh másico y fijando el Rayleigh térmico en un valor de $10^{4}$, lo anterior para poder realizar la comparación con trabajos previos (Trevisan y Bejan, 1985; 1987)

La característica más impactante del efecto de $N$ es la supresión de la convección como mecanismo de transporte: la supresión es más dramática en la vecindad de $N=1.03$, esto es, en flujos donde los efectos de flotación por temperatura y concentración son del mismo orden de magnitud y en direcciones opuestas.

La Figura 4.33 muestra el paso del flujo convectivo ocasionado por el término térmico, al flujo por fuerzas másicas y en sentido opuesto, esto es claro en las isotermas y en las líneas de concentración, observándose un movimiento de las líneas en el sentido contrario a las manecillas del reloj, este efecto es más claro en la Figura 4.34, en la cual se muestran las superficies para la componente "Z” del vector potencial, observándose como a un valor del número de flotación igual a 1 , los efectos se anulan quedando sin efecto los términos convectivos. 


\section{Pared adiabática}

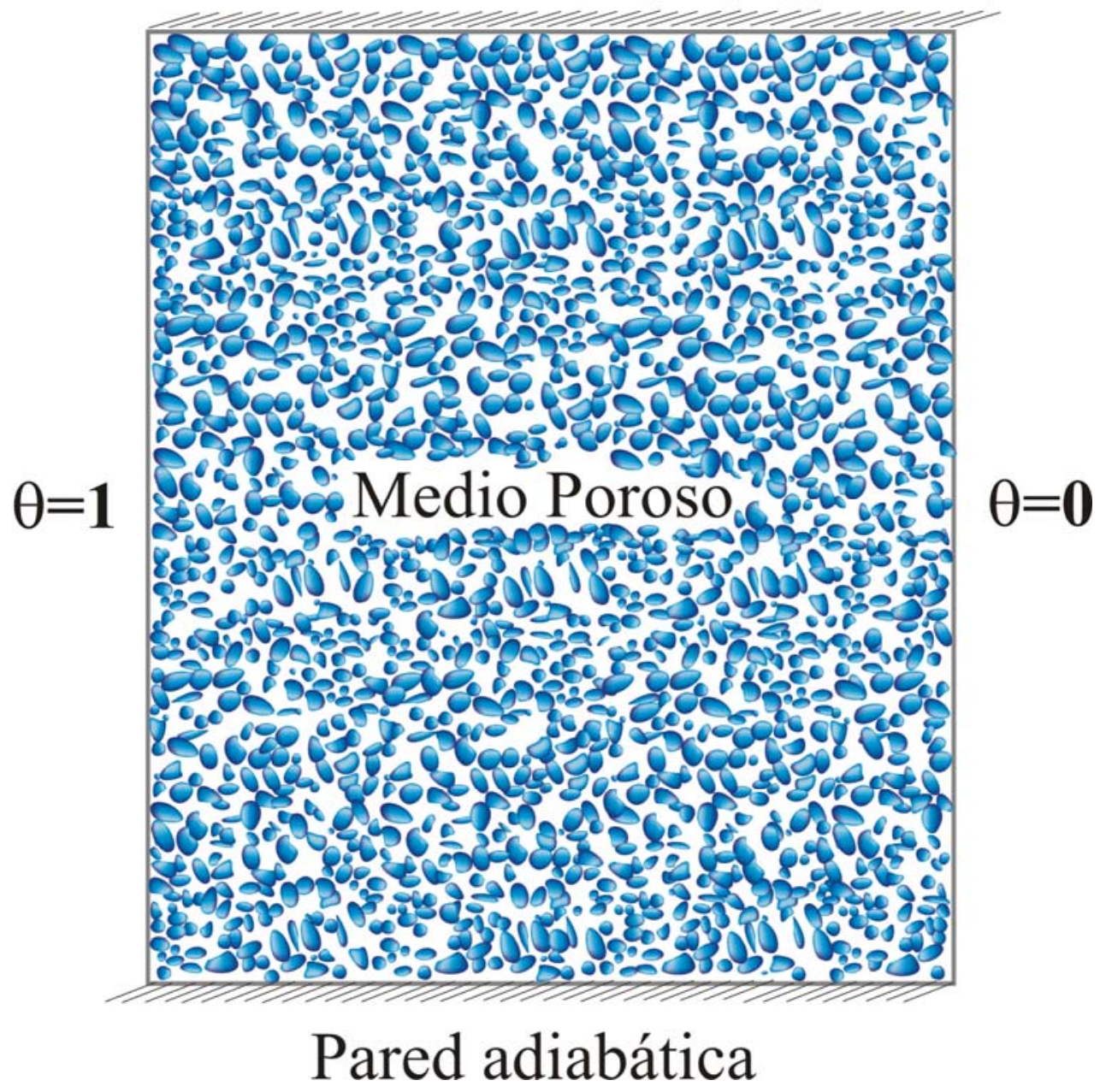

Figura 4.32.- Condiciones de frontera para el análisis de fuerzas de flotación. 

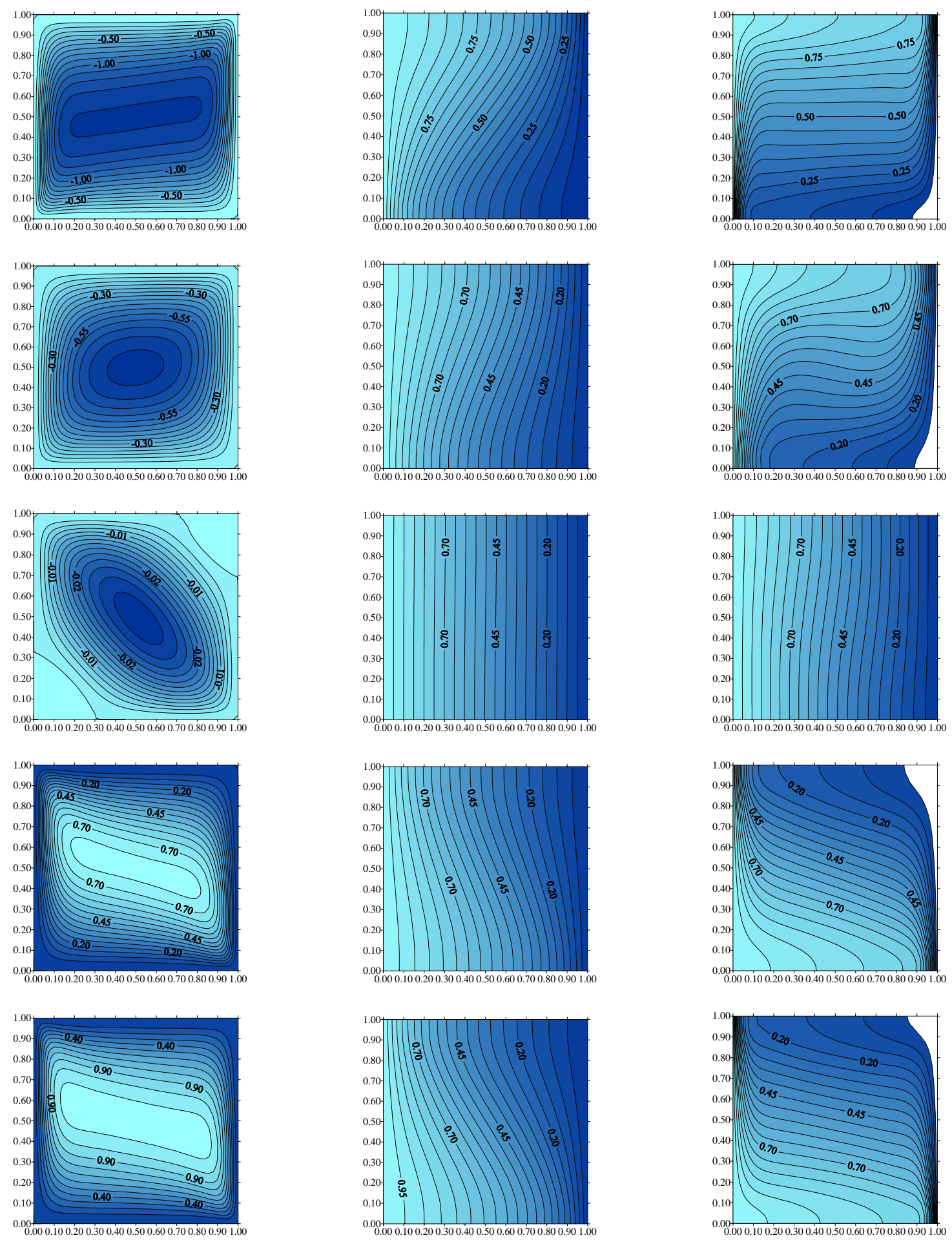

a)

b)

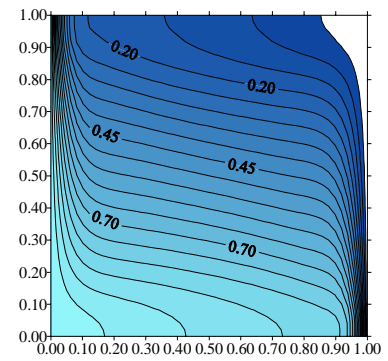

c)

Figura 4.33.- Mapas de contorno para a) Función de corriente, b)Isotermas y c)Líneas de isoconcentración, flujo inverso, $R a=10^{4}, \operatorname{Pr}=10.0, \mathrm{X}_{\mathrm{I}}=0.0$ 


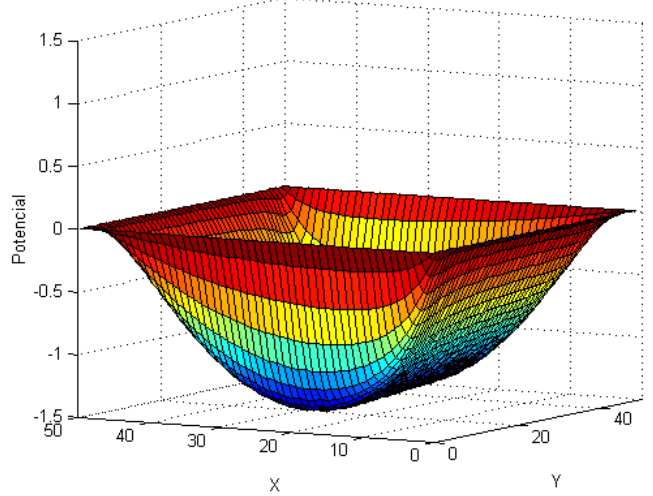

$\mathrm{N}=0.1$

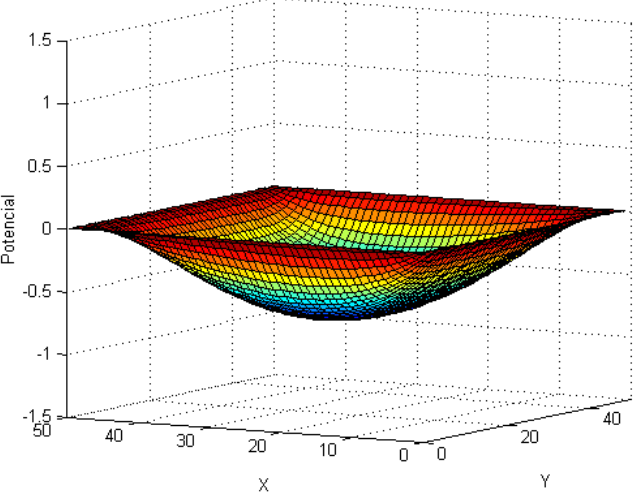

$\mathrm{N}=0.8$

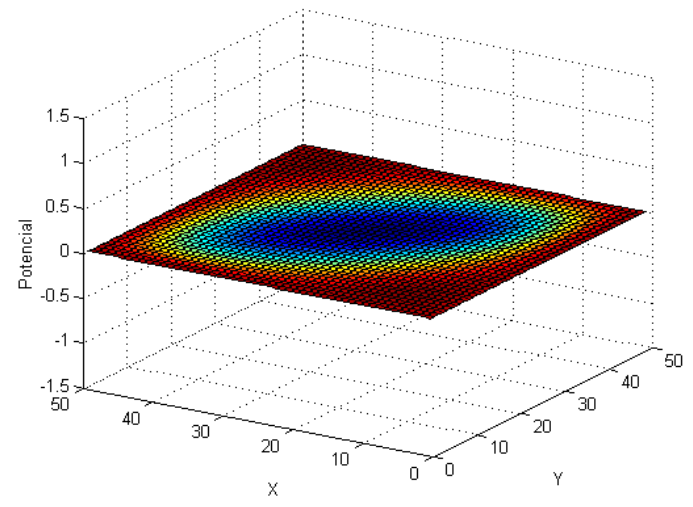

$\mathrm{N}=1.0$

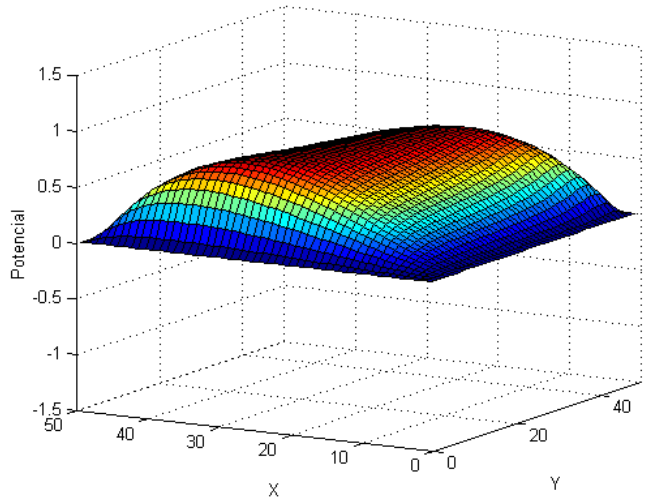

$\mathrm{N}=-0.2$

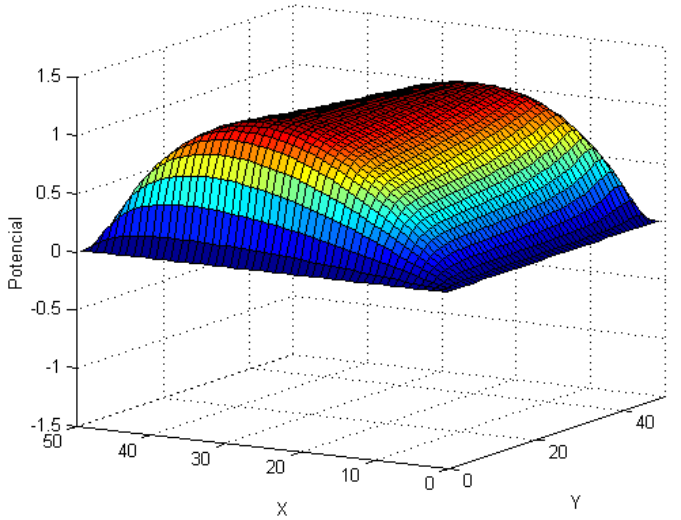

$\mathrm{N}=-0.1$

Figura 4.34.- Superficies para la función de corriente, prueba para analizar el flujo inverso. 


\section{Capítulo 5}

\section{Conclusiones y recomendaciones}

\subsection{Conclusiones}

Se presentó la formulación para el fenómeno de convección natural ocasionada por gradientes de temperatura y concentración para una cavidad rectangular en dos y tres dimensiones conteniendo dos regiones, una fluida y un medio poroso, concluyendo en la necesidad de utilizar condiciones de salto para acoplar las ecuaciones constitutivas en ambas regiones, de estas condiciones se desarrolló la que acopla la transferencia de masa, se realizaron pruebas a los modelos concluyendo que se cuenta con una herramienta confiable para posteriores estudios que consideren los fenómenos interregionales.

Se desarrolló un modelo que considera los efectos tridimensionales en la formulación vorticidad - vector potencial para el modelado de flujo convectivo en cavidades fluido-medio poroso, la alternativa que se presenta al utilizar las condiciones interregionales propuestas por Beavers y Joseph no muestra cambios significativos en cuanto al comportamiento de los perfiles de temperatura comparada con las formulaciones que utilizan la corrección de Brinkman a la ecuación de Darcy en la región porosa para cavidades rectangulares, sin embargo las comparaciones sobre los perfiles de velocidad muestran la necesidad de utilizar ecuaciones con un fundamento más sólido, sobre todo a valores grandes del número de Rayleigh, que es cuando los el término de flotación toma importancia..

De los modelos probados, se concluye que aquel que utiliza la corrección de Brinkman a la ecuación de Darcy presenta mejor estabilidad, una mejor convergencia numérica y resultados continuos en la interregión comparados con aquellos que se obtienen al utilizar las condiciones de Beavers y Joseph, lo anterior no implica una conclusión contundente en cuanto 
a su utilización, lo que queda claro con la comparación es que existen diferencias y se requiere de una solución teórica para respaldar el uso de cualquiera de los modelos presentados. Lo anterior queda respaldado con los resultados presentados por Goyeau y col. 2003, al concluir que los modelos de Brinkman y las condiciones de Beavrs y Joseph no muestran diferencias al considerarse un medio poroso homogéneo.

Se presentaron pruebas para la comparación de cuatro modelos que consideran cavidades fluido-medio poroso: dos tridimensionales y dos bidimensionales, en ellos se incluye el transporte de calor por fuerzas de flotación. En la primera de las aproximaciones el transporte de cantidad de movimiento fue modelado en términos de la ecuación de Navier Stokes y la ley de Darcy para la región fluida y porosa respectivamente. Una extensión de la condición semiempírica propuesta por Beavers y Joseph fue utilizada para acoplar las ecuaciones del fluido y del medio poroso. La segunda aproximación considera la corrección de Brinkman a la ecuación de Darcy. En este caso la continuidad de la velocidad y el vector de esfuerzos fueron usados como condiciones interfaciales.

La comparación de las predicciones entre ambas formulaciones muestran claras diferencias en los detalles de los mapas de contorno para temperatura y velocidad, Las diferencias comienzan a ser importantes cuando el número de Nusselt local y promedio es comparado para valores altos del número de Rayleigh, mostrando una clara indicación que la transferencia de calor en las paredes del contenedor predichas por ambos modelos muestran grandes diferencias cuando el $R a$ y $D a$ son suficientemente grandes.

Se cuenta con una formulación confiable para hacer las pruebas a la condición de salto que se desarrolló, así como las ya existentes, este modelo tiene la limitante de que considera el estado estacionario, pero la estabilidad del mismo en la validación de las condiciones de salto será de gran ayuda para tener el modelo final.

Se realizó un análisis para la ecuación de transferencia de masa (considerando difusión y adsorción) en la frontera entre un fluido homogéneo y un medio poroso, partiendo de las ecuaciones usualmente utilizadas, el desarrollo ha impuesto la condición de continuidad en la frontera para la concentración del soluto y se derivó una condición de salto para el flux que asegura el cumplimiento de la ecuación generalizada para el transporte de masa. 


\subsection{Perspectivas.}

Con el modelo tridimensional se pretende hacer pruebas para determinar la influencia del término convectivo en la ecuación de transferencia de cantidad de movimiento en la región porosa, además de la inclusión de la corrección de Fochheimer (Whitaker, 1996) a la ecuación de Darcy en la ecuación de transferencia de cantidad de movimiento en la región porosa.

El modelo no considera los cambios de porosidad en las proximidades de las paredes por lo que se sugiere desarrollar las condiciones de salto para la interregión sólido-medio poroso.

Los resultados muestran la necesidad de un programa experimental para aclarar cual es la forma correcta de modelar la ecuación de transferencia de cantidad de movimiento en un medio poroso. El contar con el programa experimental podría ser orientado a obtener medidas de la velocidad temperatura promedio.

Con el modelo tridimensional se puede llevar a cabo la evaluación de la transferencia de calor y masa mediante el Número de Nusselt y Sherwood local y global en la interregión fluido-medio poroso y con ello la evaluación de coeficientes de transferencia de calor y masa interregionales.

Parte importante del desarrollo del modelo tridimensional es probar las condiciones de salto entre un fluido y un medio poroso previamente desarrolladas para las ecuaciones de transferencia de energía, cantidad de movimiento y transferencia de masa. Se pretenden realizar dichas pruebas utilizando el modelo mostrado en el presente trabajo. 


\section{Bibliografía:}

Alazmi B. and Vafai K., 2001, "Analisys of Fluid Flow and Heat Transfer Interfacial Conditions Between a Porous Medium and a Fluid Layer”, International Journal of Heat and Mass Transfer, 44, 1735-1749.

Ames, W. F., 1992, “Numerical Methods for Partial Differential Equations”, Academics Press., USA.

Aris, R., 1959, “The Longitudinal Diffusion Coefficient in Flow Through a Tube with Stagnant Pockets”, Chemical Engineering Science, 11, 194-198.

Aziz K. and Hellums J. D., 1985, "Numerical Solution of the Dimensional Equation of Motion for Laminar Natural Convection”, Phys. Fluids, 10, 314-324.

Balakotaiah, V. and Luss D., 1981, "Effect of Flow Direction on Conversion in Isothermal Radial Flow Fixed-bed Reactors ”, AIChE Journal, 27, 442-450.

Barakos G. K. Mitsoulis E. and Assimacopoulos D., 1994, "Natural Convection Flow in a Square Cavity Revisited: Laminar and Turbulent Models with Wall Functions”, International Journal for Numerical Methods in Fluids, 18, 695-719.

Batchelor G. K., 1954, "Heat Transfer by Free Convection Across Cavity Between Vertical Boundaries at Different Temperatures”, Quarterly of Applied Mathematics, 12, 209-233.

Bera P. and Khalili., 2002, "Double-diffusive Natural Convection in an Anisotropic Porous Cavity with Opposing Bouyancy Forces: Multi-Solutions and Oscillations”, International Journal of Heat and Mass Transfer, 45, (15), 3205-3222.

Beavers G. S. and Joseph D. D., 1967, "Boundary Conditions at a Naturally Permeable Wall”, Journal of Fluid Mech., 30, 197-207.

Beckermann C., Ramadhyani S. and Viskanta R., 1987, "Natural Convection Flow and Heat Transfer Between a Fluid and a Porous Layer Inside a Rectangular Enclosure”, ASME Journal of Heat Transfer, 109, 363-370.

Beckermann C., Viskanta R. and Ramadhyani S., 1988, "Natural Convection in Vertical Enclosures Containing Simultaneously Fluid and Porous Layers”, Journal of Fluid Mechanics, 186, 257-284.

Behnia M.; Stella F. and Guj G., 1995, “A Numerical Study of Three-Dimensional Combined Bouyancy and Thermocapillary Convection”, International Journal of Multipase Flow, 27, (5), 717. 
Bejan A. and Poulikakos D., 1984, “The Non-Darcy Regime for Vertical Boundary Layer Natural Convection in a Porous Medium”, International Journal of Heat and Mass Transfer, 27, (5), 717.

Bennacer R. and Gobin D., 1996, “Cooperating Thermosolutal Convection in Enclosures -I. Scale Analysis and Mass Transfer”, International Journal of Heat and Mass Transfer, 39, (13), 2671-2681.

Bian W., Vasseur P. and Bilgen E., 1994, "Boundary-Layer Analysis for Natural Convection in a Vertical Porous Layer Filled with a Non-Newtonian Fluid”, International Journal of Heat and Fluid Flow, 15, (5), 384-391.

Bird R. Byron, Steward Warren E. and Lightfoot Edwin N., 2002, “Transport Phenomena”, John Wiley and Sons Inc., $2^{\text {nd }}$ Edition, USA.

Brinkman H. C., 1947, "A Calculation of Viscous Force Excerted by Flowing Fluid on a Dense Swarm of Particles”, Applied Scientific Research, A1, 27-34.

Brockwell P. J. and Davids R. A., 2002, "Introduction to Time Series and Forecasting", Springer., $2^{\text {nd }}$ Edition, USA.

Bubnovich V., Rosas C., Santander R. and Cáceres G., 2002, “Computation of Transsient Natural Convection in a Square Cavity by an Implicit Finite-Difference Acheme in Terms of the Stream Function and Temperature", Numerical Heat Transfer Part A: Applications, 42, (4), 401-425.

Carbonell R. G. and Whitaker S., 1983, “Dispersion in Pulsed Systems II: Theoretical Developments for Passive Dispersion in Porous Media ", Chemical Engineering Science, 38, 1975-1802.

Carbonell R. G. and Whitaker S., 1984, "Heat and Mass Transport in Porous Media”, Mechanics of Fluid in Porous Media, J Bear y MY Corapcioglu (Eds) (Martinus Nijhoff, Bruselas, 1984) 121-198.

Catton I., 1978, "Natural Convection in Enclosures ", Proc. $6^{\text {th }}$ Int. Heat Transfer Conf., 6, 1331.

Ciofalo M. and Karayiannis T. G., 1991, "Natural Convection Heat Transfer in a Partially -or Complety- Partitioned Vertical Rectangular Enclosures”, International Journal of Heat and Mass Transfer, 34, 167-179.

Chakraborty S. and Dutta P., 2003, "Three-Dimensional Double-Diffusive Convection and Macrosegregation During Non-equilibrium Solidification of Binary Mixtures”, International Journal of Heat and Mass Transfer, 46, 2115-2134.

Chamkha A.J., 2003, "Double-diffusive Convection in a Porous Enclosure with Cooperating Temperature and Concentration Gradients and Heat Generation or Adsorption Effects” Numerical Heat Transfer, Part A, 41, 65-87.

Chan B. K. C., Ivey C. M. and Barry J. M., 1970, "Natural Convection in Enclosed Porous Media with Rectangular Boundaries”, ASME Journal of Heat Transfer, 92, 21-27.

Chen H.-L. and Hou K. C., 1985, “Bioseparation by Cartridge Chomatography.”, In Annual Reports on Fermentation Processes (Edited by G. T. Tsao), 8, 59-71, Academic Press, New York.

Chen F. and Chen C. F., 1988, "Onset of Finger Convection in a Horizontal Porous Layer Underlying a Fluid Layer ”, ASME Journal of Heat Transfer, 110, 403-409. 
Colomer G., Costa M., Consul R. and Oliva A., 2004, "Three-Dimensional Numerical Simulation of Convection and Radiation in a Differentially Heated Cavity Using the Discrete Ordinates Method”, International Journal of Heat and Mass Transfer, 42,(2), 257-269.

Costa V. A. F., 2004, "Double-Diffusive Natural Convection in Parallelogrammic Enclosures Filled with Fluid-Satured Porous Media”, International Journal of Heat and Mass Transfer, 47, 2699-2714.

Dagtekin I. and Oztop H. F., 2001, "Natural Convection Heat Transfer by Heated Partitions within Enclosure", International Communications in Heat and Mass Transfer, 28, (6); 823-834.

Dai W., Zhang Y. and Nassar R., 2000, “A Hybrid Finite Element-Alternating Direction Implicit Method for Solving Parabolic Differential Equations on Multilayers with Irregular Geometry”, Journal of Computational and Applied Mathematics, 117, (1), 116.

Dash R. K., Mehta K.N. and Jayaraman G., 1996, "Casson Fluid flow in a Pipe Filled with a Homogeneous Porous Medium”, International Journal of Engineering Science, 34, 1145-1156.

De Lemos M. J. S. and Mesquita M.S.Kalla., 2003, “Turbulent Mass Transport in Satured rigid Porous Media”, International Communications in Heat Mass Transfer, 30, (1), 105-113.

Diersch H.-J. G. and Kolditz O., 1998, “Coupled Groundwater Flow and Transport: 2. Thermohaline and 3D Convection Systems”, Advances in Water Resources, 21, (8), 401425.

Ding H., Shu C., Yeo K. S. and Xu D., 2004, “Development of Least-Square-Based twoDimensional finite-Difference Schemes and their Application to Simulate Natural Convection in a Cavity”, Computers \& Fluids, 33, 137-154.

Dong S.-F. and Li Y.-T., 2004, “ Conjugate of Natural Convection and Conduction in a Complicated Enclosure ”, International Journal of Heat and Mass Transfer, 47, 22332239.

Duval F., Fichot F. and Quintard M., 2004, “A Local Thermal Non-equilibrium Model for Two-Phase Flows Phase-Change in Porous Media”, International Journal of Heat and Mass Transfer, 47, 613-639.

Ettefagh J., Vafai K. and Kim S. J., 1991, "Non-Darcian Effects in Open-Ended Cavities Filled with a Porous Medium”, ASME Journal of Heat Transfer, 113, 747-756.

Fusegi T. and Hyun J. M., 1994, "Laminar and Transitional Natural Convection in an Enclosure with complex and Realistic Conditions”, International Journal of Heat and Fluid Flow, 15, (4), 258-268.

Gebhart B., Jaluria Y., Mahajan R. L. and Sammakia B., 1988, "Bouyancy-induced Flows and Transport”, Hemisphere Publishing Corporation, New York.

Gibbs J. W., 1928, “The Collected Works of J. Willard Gibbs”, Vol 1, Yale University Press, New Haven, Connecticut.

Gobin D., Goyeau B. and Neculae A., 2005, "Convective Heat and Solute Transfer in Partially Porous Cavities”, International Journal of Heat and Mass Transfer, 48, 1898-1908. 
Gobin D., Goyeau B. and Songbe J. -P., 1998, "Double Diffusive Natural Convection in a Composite Fluid-Porous Layer”, Journal of Heat Transfer, 120, 234-242.

Goyeau B., Lhuillier D., Gobin D., and Velarde M. G., 2003, “Momentum Transport at a Fluid-Porous Interface”, International Journal of Heat and Mass Transfer, 46, 40714081.

Gray W. G., 1975, “A Derivation of the Equations for Multi-Phase Transport ”, Chemical Engineering Science, 30, 229-233

Gray D. D. and Giorgini A., 1976, "The Validity of the Boussinesq Aproximation for Liquids and Gases ”, International Journal of Heat and Mass Transfer, 19, 545-551.

Greenkorn R. A., 1983, "Flow Phenomena in Porous Media”, Marcel Dekker Inc. N.Y..

Haddad O.M., Al-Nimr M.A. and Al-Khateeb A.N., 2004, "Validation of the Local Thermal Equilibrium Assumtion in Natural Convection from a Vertical Plate Embedded in Porous Medium: Non-Darcian Model”, International Journal of Heat and Mass Transfer, 47, 2037-2042.

Hanjalic K., 1994, “Advanced Turbulence Closure Models: a View of Current Status and Future Prospects”, International Journal of Heat and Fluid Flow, 15, (3), 178-203.

Hellums J. D. and Churchill S. W., 1962, "Transient and Steady State Free and Natural Convection, Numerical Solutions”, AIChE Journal, 8, 690-695.

Hickox C. E., Gartling D. K., Givler R. C. and Altobelli S. A., 1996, “Issues Involved in the Numerical Simulation of Coupled Viscous and Porous Flow ”, International Conference on Porous Media, June 21, Kona, Hawaii.

Hirt C. W. and Cook J. L., 1972, "Transient Three-Dimensional Natural Convection in Confined Porous Medium”, International Journal Heat and Mass Transfer, 15, 73-89.

Hirasaki G. J. and Hellums, 1968, “A General Formulation of the Boundary Conditions on the Vector Potential in Three-Dimensional Hydrodynamics”, Qnarterly od Applied Mathematics, XXVI, 331-342.

Hirasaki G. J. and Hellums, 1970, "Boundary Conditions on the Vector and Scalar Potentials in Viscous Three-Dimensional Hydrodynemics”, Qnarterly od Applied Mathematics, XXVIII, 293-297.

Holst P. H. and Aziz K., 1972, “Transient Three-Dimensional Natural Convection in Confined Porous Medium”, International Journal of Heat and Mass Transfer, 15, 73-89.

Hoogendoorn C. J., 1986, “Natural Convection in Enclosures”, Proc. $8^{\text {th }}$ Int. Heat Transfer Conf., 1, 111-120.

Howes F. A. and Whitaker S., 1985, “The Spatial Averaging Theorem Revisited”, Chemical Engineering Science, 40, 1387-1392.

Hsu C.T. and Cheng P., 1985, “The Brinkman Model for Natural Convection about a Semiinfinite Vertical Flat Plate in a Porous Medium”, International Journal of Heat and Mass Transfer, 28, (3), 683.

Huang H. S., Roy S., Hou K. C. and Tsao G. T., 1988, "Scaling-up of Affinity Chromatography by Radial-flow Cartridges ”, Biotechnol. Prog., 4, 159-165.

Ingam D.B. and Pop I., 1998, “Transport Phenomena in Porous Media”, Pergamon 
Jiménez-Islas H., Navarrete-Bolaños J.L. y Botello-Álvarez E., 2004, “Estudio Numérico de la Convección Natural de Calor y Masa 2-D en Granos Almacenados en Silos Cilíndricos”, Agrociencia, 38, (3), 325-342.

Jiménez Islas Hugo, 1999, “Modelamiento Matemático de los Procesos de Transferencia de Momentum, Calor y Masa en Medios Porosos”, Tesis de Doctorado Universidad Autónoma Metropolitana-Iztapalapa, México.

Jiménez-Islas H., López-Isunza F. and Ochoa-Tapia J.A., 1999, "Natural Convection in a Cylindrical Porous Cavity with Internal Heat Source: A numerical Study with Brinkman-Extended darcy Model”, International Journal of Heat and Mass Transfer, 42, 4185-4195

Kalla L., Vasseur P., Beji H. and Duval R., 2001a, "Double Diffusive Convection within a Horizontal Porous Layer Salted from the Bottom and Heated Horizontally", International Communications in Heat Mass Transfer, 16, (1), 1-10.

Kalla L., Mamou M., Vasseur P. and Robillard L., 2001b, "Multiple Solutions for DoubleDiffusive Convection in a Shallow Porous Cavity with Vertical Fluxes of Heat and Mass”, International Journal of Heat and Mass Transfer, 44, 4493-4504.

Kaviany M., 1991, "Principles of Heat Transfer in Porous Media”, Springer, New York.

Kelkar K. M. and Patankar S. V., 1990, "Numerical Prediction of Natural Convection in Square Partitioned Enclosures”, Journal of Fluid Mechanics, 17, 269-285.

Khan A. A. and Zebib A., 1981, "Double Diffusive Instabilitiy in a Vertical Layer of a Porous Medium”, ASME Journal of Heat Transfer, 103, 553-560.

Khaled A.-R.A. and Vafai K., 2003, "The Role of Porous Media in Modeling flow and Heat Transfer in Biological Tissue”, International Journal of Heat and Mass Transfer, 46, 4989-5003.

Kim S. J. and Vafai K., 1989, "Analysis of Natural Convection about a Vertical Plate Embedded in a Porous medium”, International Journal of Heat and Mass Transfer, 32, (4), 665.

Kuznetsov A. V., 1996, “Analytical Investigation of the Fluid Flow in the Interface Region Between a Porous Medium and a Clear Fluid in Channels Partially Filled With a Porous Medium”, Applied Scientific Research, 56, 53-67.

Kuznetsov A. V., 1997, "Influence of the Stress Jump Condition at the PorousMedium/Clear-Fluid Interface on a Flow at a Porous Wall”, International Communications in Heat Mass Transfer, 24, 401-410.

Kuznetsov A. V., 1998a, "Analytical Investigation of Couette Flow in a Composite Channel Partially Filled With a Porous Medium and Partially Filled with a Clear Fluid”, International Journal of Heat and Mass Transfer, 41, 2556-2560.

Kuznetsov A. V., 1998b, "Analytical Study of Fluid flow and Heat Transfer During Forced Convection in a Composite Chennel Partly Filled With a Brinkman-Forchheimer Porous Medium, Flow”, Turbulence Combust, 60, 173-192.

Kuznetsov A. V., 1999, "Fluid Mechanics and Heat Transfer in the Interface Region Between a Porous Medium and a Fluid Layer: A Boundary Layer Solution”, Journal of Porous Media, 2, (3), 309-321. 
Kuznetsov A. V. and Jiang N., 2001, "Numerical Investigation of Bioconvection of Gravitactic Microorganisms in an isotropic Porous Medium”, International Communications in Heat Mass Transfer, 28, 877-886.

Kuznetsov A. V. and Avramenko A. A., 2002, “A 2D Analysis of Stability of Bioconvection in a Fluid Saturated Porous Medium-Estimation of the Critical Permeability Value”, International Communications in Heat Mass Transfer, 29, 175-184.

Lapidus L. and Pinder G. F., 1982, “Numerical Solutions of Partial Differential Equations in Science and Engineering”, John Wiley and Sons, New York, USA.

Lauriat G. and Prasad V., 1987, "Natural Convection in a Vertical Porous Cavity: a Numerical Study for Brinkman-Extended Darcy Formulation”, ASME Journal of Heat Transfer, 109, 687-696.

Lauriat G., 1989, "Non-Darcian Effects on Natural Convection in a Vertical Porous Enclosure”, International Journal of Heat and Mass Transfer, 22, (11), 2135-2148.

Le Peutrec Y. and Lauriat G., 1990, "Effects of the Heat Transfer at the Slide Wall on Natural Convection in Cavities”, ASME, Journal of Heat Transfer, 112, 370-378

Lee J., Hyun M. T and Kim K.W., 1988, "Natural Convection in Confined Fluid With Combined Horizontal Temperature and Concentration Gradient”, International Journal of Heat and Mass Transfer, 31, 1969.

Lee W.-C., Tsai, G.-J. and Tsao G. T., 1990, "Radial-flow Affinity Chromatography for Trypsin Purification”, ACS Symp. Ser., 427, 104-117.

Lenhoff A. M. and Lightfoot E. N., 1984, “ The Effects of axial Diffusion and Permeability Barriers on the transient response of tissue cylinders. II. Solution in time domain”, $J$. theor. Biol., 106, 207-238.

Lenhoff A. M. and Lightfoot E. N., 1986, "Convective Dispersion and Interphase Mass Transfer”, Chemical Engineering Science, 41, (11), 2795-2810.

Leonardi E., 1984, "A Numerical Study of the Effects of Fluid properties on Natural Convection”, Ph.D. thesis, School of Mechanical and Industrial Engineering, The University of New South Wales.

Liao S. J., 1995, “An Approximate Solution Technique which does not Depend upon Small Parameters: A Special Example”, International Journal of Non-linear Mechanics, 30, 371-380.

Liao S. J., 1997, “A Kind of Approximate Solution Technique which does not Depend upon Small Parameters (part 2): An Application in Fluid Mechanics”, International Journal of Non-linear Mechanics, 32, 815-822.

Liao S. J., 1999a, “A Uniformly valid Analytic Solution of Two-dimensional Viscous flow over a semi-infinite flat Plate”, Journal of Fluid Mechanics, 385, 101-128.

Liao S. J., 1999b, “An Explicit, Totally Analytic Approximate Solution for Blasius’ Viscous flow Problems”, International Journal of Non-linear Mechanics, 34, 759-778.

Liao S. J., 2002, “An Analytic Approximation of the Drag Coefficient for the Viscous flow past a Sphere”, International Journal of Non-linear Mechanics, 37, 1-18.

Liao S. J. and Campo A., 2002, "Analytic Solutions of the Temperature Distribution in Blasius Viscous flow Problems”, Journal of Fluid Mechanics, 453, 411-425. 
Mahidjiba A., Mamou M. and Vasseur P., 2000, “Onset of Double-Diffusive Convection in a Rectangular Porous Cavity Subject to Mixed Boundary Conditions”, International Journal of Heat and Mass Transfer, 43, 1505-1522.

Mallinson G. D. and De Vahl Davis G., 1973, "The Method of the False Transient for the Solution of Coupled Elliptic Equations”, Journal of Computational Physics, 12, 435461.

Mallinson G. D. and De Vahl Davis G., 1977, “Three-Dimensional Natural Convection in a Box: a Numerical Study”, Journal of Fluid Mechanics., 83, 1-31.

Mamou M., Vasseur P. and Hasnaoui M., 2001, “On Numerical Stability Analysis of DoubleDiffusive Convection in Confined Enclosures”, Journal of Fluids Mechanics, 433, 209250.

Mamou M., Vasseur P. and Bilgen E., 1998, "Double-diffusive Convection Instability in a Vertical Porous Enclusure”, Journal of Fluids Mechanics, 368, 263-289.

Martini W. R. and Churchill S. W., 1960, "Natural Convection Inside a Horizontal Cylinder”, AIChE Journal, 6, 251-257.

Martins-Costa M. L. and Saldanha de Gama R. M., 1994, "A Local Model for the Heat Transfer Process in Two Distinct Flow Regions”, International Journal of Heat and Fluid Flow, 15, (6), 477-485.

Murray B. T. and Chen C. F., 1989, “Double-Diffusive Convection in a Porous Medium”, Journal of Fluids Mechanics, 201, 147-166.

Neale G. and Nader W., 1974, "Practical Significance of Brinkman's Extension of Darcy's Law: Coupled Parallel Flows within a Channel and Bounding Porous Medium”, Can. J. Chem. Engrg., 52, 475-478.

Nazar R., Amin N., Filip D. and Pop I., 2003, “The Brinkman Model for the Mixed Convection Boundary Layer Flow Past a Horizontal Circular Cylinder in a Porous Medium”, International Journal of Heat and Mass Transfer., 46, (17), 3167-3178.

Nield D. A., 1968, “Onset of Thermohaline Convection in a Porous Medium”, Water Resources Research, 4, 553-560.

Nield D. A., 1977, "Onset of Convection in a Fluid Layer Overlaying a Layer of Porous Medium”, Journal of Fluid Mechanics., 81, 513-522.

Nield D. A. and Bejan A., 1992, “Convection in Porous Media”, Springer, New York.

Nield D. A., Kuznetsov A.V. and Avramenko A. A., 2004, "The Onset of Bioconvection in a Horizontal Porous-Medium Layer”, Transport in Porous Media, 54, 335-344.

Nishimura T., Takumi T., Shiraishi M., Kawamura Y. and Ozoe H., 1986, "Numerical Analysis of Natural convection in a Rectangular enclosure Horizontally Divided into Fluid and Porous Regions”, International Journal of Heat and Mass Transfer, 29, 889898.

Nozad I., Carbonell R. G. and Whitaker S., 1985, “Heat Conduction in Multiphases Systems: Theory and Experiment for Two-Phase Systems", Chemical Engineering Science, 40, (5), 843-855.

Ochoa-Tapia J. A., Del Río P. J. A. and Whitaker S., 1993, "Bulk and Surface Diffusion in Porous Media: An Application of the Surface Averaging Theorem”, Chem. Engng. Sci., 48, 2061-2082. 
Ochoa T. J. A. y Soria A., 1995, "Emulsiones Dobles: Condiciones de Salto para el Transporte de Masa en la Frontera entre la Región Membrana y la Región Externa”, XVI Encuentro Nacional de la Ecademia de Docencia e Investigación en Ingeniería Química (AMIDIQ).

Ochoa T. J. A., Stroeve P. and Whitaker S., 1994, "Diffusive Transport in Two-Phase Media: Spatially Periodic Models and Maxwel's Theory for Isotropic and Anisotropic Systems" , Chemical Engineering Science, 49, (5), 709-726.

Ochoa T. J. A. and Whitaker S., 1995a, "Momentum Transfer at the Boundary Between a Porous Medium and a Homogeneous Fluid-I. Theoretical Development”, International Journal of Heat and Mass Transfer, 38, (14), 2635-2646.

Ochoa T. J. A. and Whitaker S., 1995b, "Momentum Transfer at the Boundary Between a Porous Medium and a Homogeneous Fluid-II. Comparison with Experiment”, International Journal of Heat and Mass Transfer, 38, (14), 2647-2655.

Ochoa T. J. A. y Whitaker S., 1995c, "El Método del Promedio Volumétrico para el Estudio de Difusión en Sistemas de Varias Componentes”, Revista Mexicana de Física, 41, (3), 451-470.

Ochoa T. J. A. and Whitaker S., 1997, "Heat Transfer at the Boundary Between a Porous Medium and a Homegeneous Fluid ”, Int. J. Heat Mass Transfer, 40, (11), 2691-2707.

Ochoa-Tapia J. A. and Whitaker S., 1998a, “ Momentum Jump Condition at the Boundary Between a Porous Medium and a Homogeneous Fluid: Inertial Effects”, Journal of Porous Media, 1, 201-217.

Ochoa T. J. A. and Whitaker S., 1998b, “ Heat Transfer at the Boundary Between a Porous Medium and a Homegeneous Fluid: The One-Equation Model”, Journal of Porous Media, 1, (1) 31-46.

Ostrach J., 1967, “Completely Confined Natural Convection”, Developments in Mechanics, Proc. Tenth Midwestern Mechanics Conf., 4, Johnson, Chicago, IL, 53-81.

Ostrach J., 1988, "Natural Convection in Enclusures”, ASME Journal of Heat Transfer, 110, 1175-1190.

Ozoe H. Yamamoto K. and Churchill S. W., 1979, “Three- Dimensional Numerical Analysis of Natural Convection in an Inclined with a Square Cross Section”, AIChE Journal, 25, (4), 709-716.

Patankar S. V., 1980, “Numerical Heat Transfer and Fluid Flow”, Hemisphere, Washington, D.C., USA.

Peaceman D.W. and Rachford D.D., 1955, “The Numerical Solutions of Parabolic and Elliptic Differential Equations”, Society for Industrial and Applied Mathematics Journal, 3, 2847.

Peng Y., Shu C. and Chew Y.T., 2003, “A 3D Incompressible Thermal Lattice Boltzmann Model and its Application to Simulate Natural Convection in a Cubic Cavity”, Journal of Computational Physics, 193, 206-274.

Plumb O. A. and Huenefeld J. C., 1981, "Non-Darcy Natural Convection from Heated Surfaces in Saturated Porous Media”, International Journal of Heat and Mass Transfer, 24, (4) 765.

Poots G., 1962, "Heat Transfer by Laminar Free Convection in Enclosed Plane Gas Layers ”, Quarterly of Applied Mathematics, 11, 257-273. 
Postelnicu A., 2004, "Influence of a Magnetic Field on Heat and Mass Transfer by Natural Convection from Vertical Surfaces in Porous Media Considering Soret and Dufuor Effects”, International Journal of Heat and Mass Transfer, 47, 1467-1472.

Poujol F.T., Rojas J. and Ramos E., 2000, "Natural Convection of a High Prandtl Number in a Cavity”, International Communications in Heat Mass Transfer, 27, (1), 109-1118.

Poulikakos D., Bejan A., 1983, “Unsteady Natural Convection in a Porous Layer”, Phys. Fluids, 26, 1183-1191.

Poulikakos D., 1986, “Double Diffusive Convection in a Horizontal Sparsely Porous Layer”, International Communications in Heat Mass Transfer, 13, 587-598.

Poulikakos D., Bejan A., Selimos B. and Blake K. R., 1986, "High Rayleigh Number Convection in a Fluid Overlaying a Porous Bed”, International Journal of Heat and Fluid Flow, 7, 109-116.

Prasad V., 1987, "Thermal Convection in a Rectangular Cavity Filled With a HeatGenerating, Darcy Porous Medium”, ASME Journal of Heat Transfer, 109, 697-703.

Prasad V. and Chui A., 1989, "Natural Convection in a Cylindrical Porous Enclosure With Internal Heat Generation”, ASME Journal of Heat Transfer, 111, 916-925.

Prasad V. and Kulacki F. A., 1984a, "Convective Heat Transfer in a Rectangular Porous Cavity. Effect on Aspect Radio on Flow Structure and Heat Transfer”, ASME Journal of Heat Transfer, 106, 158-165.

Prasad V. and Kulacki F. A., 1984b, "Natural Convection in a Rectangular Porous Cavity with Constant Heat Flux on one Vertical Wall”, ASME Journal of Heat Transfer, 106, 152-157.

Prasad V., Kulacki F. A. and Keyhani M., 1985, "Natural Convection in Porous Media”, Journal of Fluid Mechanics, 150, 89-119.

Preziosi L. and Farina A., 2002, “On Darcy's law for Growing Porous Media ”, International Journal of Non-linear Mechanics, 37, 485-491.

Provitsky A., 2002, "Parallel ADI Solver Based on Processor Scheduling”, Applied Mathematicxs and Computation, 133, (1), 43-81.

Provitsky A. and Morris P. J., 2000; "A Higher-Order Compact Method in Space and Time Based on Parallel Implementation of the Thomas Algorithm”, Journal of Computational Physics, 161, (1), 182-203.

Prud'homme M., Bougherara H. and Bahloul A., 2003, "Convection in a Vertical Cavity Submitted to Crossed Uniform Heat Fluxes”, International Journal of Heat and Mass Transfer, 46, 3831-3840.

Quintard M. and Whitaker S., 1990, “Two-Phase Flow in Heterogeneous Porous Media I: The Influence of Large Spatial and Temporal Gradients”, Transport in Porous Media, 5, 341-379.

Quintard M. and Whitaker S., 1994a, “Transport in Ordered and Disordered Porous Media I: The Cellular Average and the Use of Weighting Functions”, Transport in Porous Media, 14, 163-177.

Quintard M. and Whitaker S., 1994b, “Transport in Ordered and Disordered Porous Media II: Generalized Volume Averaging”, Transport in Porous Media, 14, 177-206. 
Quintard M. and Whitaker S., 1994c, “Transport in Ordered and Disordered Porous Media III: Closure and Comparison Between Theory and Experiment”, Transport in Porous Media, 15, 31-49.

Quintard M. and Whitaker S., 1994d, “Transport in Ordered and Disordered Porous Media IV: Computer Generated Porous Media for Three-Dimensional Systems”, Transport in Porous Media, 15, 51-70.

Quintard M. and Whitaker S., 1994e, “Transport in Ordered and Disordered Porous Media V: Geometrical Result for Two-Dimensional Systems”, Transport in Porous Media, 15, 183-196.

Quintard M. and Whitaker S., 1995, "Local Thermal Equilibrium for Transient Heat Conduction I: Theory and Comparison with Numerical Experiment”, International Journal of Heat and Mass Transfer, 38, 2779-2796.

Raul R., Bernard P. S. and Buckley F. T. Jr., 1990, "An Aplication of the Vorticity-Vector Potential Method to Laminar Cube Flow”, International Journal for Numerical Methods in Fluids, 10, 875-888.

Ravi M. R., Henkes R. A. W. M. and Hoogendoorn C. J., 1994, "On the High-RayleighNumber Structure of Steady Laminar Natural-Convection Flow in a Square Enclosure”, Journal of Fluid Mechanics, 262, 325-351.

Roache P.J., 1972, “Computational Fluid Dynamics”, Hermosa Publishers, Albuquerque, N.M., USA.

Rosenberg N. D. and Spera F. J., 1992, "Thermohaline convection in a porous media heated from below”, International Journal of Heat and Mass Transfer, 35, 1261-1273.

Rudraiah N., Srimani P.K. and Friedrich R., 1982, "Finite Amplitude Convection in a twoComponent fluid Saturated Porous Layer”, International Journal of Heat and Mass Transfer, 25, 715-722.

Saeid N.H. and Pop I., 2004, "Transient Free Convection in a Square Cavity Filled with a Porous Medium”, International Journal of Heat and Mass Transfer, 47, 1917-1924.

Samarskii A. A. and Andreyev V. B., 1963, "On a high-accurancy diference scheme for an elliptic equation with several space variables”, URSS Comp Math. And Math. Phys, 3, (6), 1373-1382.

Sathe S. B., Lin W.-Q. and Tong T.W., 1988, "Natural Convection in Enclosures Containing and Insulation With a Permeable Fluid-Porous Interface”, International Journal of Heat and Fluid Flow, 9 ,(4), 389-395.

Samuels R.M. and Churchill W.S., 1967, "Stability of Fluid in a Rectangular Region Heated From Below”, AIChE Journal, 13, (1), 77-85.

Saxena V. and Weil, A. E., 1987, "Radial Flow Columns a New Aproach to Scaling-up Biopurifications”, BioChromatography, 2, 90-97.

Saxena V., Weil A. E., Kawahata R. T., McGregor W. C. and Chandler M., 1987, "Applications of Radial-flow Columns for Fast Affinity Chromatography", Am. Lab. (Fairfield, Conn.), 19, 112-120.

Sezai I. and Mohamad A. A., 1999, "Three-dimensional Double-Diffusive Convection in a Porous Cubic Enclosure due to Opposing Gradients of Temperature and Concentration”, Journal of Fluids Mechanics, 400, 333-353. 
Sezai I. and Mohamad A. A., 2000, "Double Diffusive Convection in a Cubic Enclosure with Opposing Temperature and Concentration Gradients”, Physics of Fluids, 12, (9), 22102223.

Shih T.C., Kou H.S. and Lin W. L., 2002, "Effect of Effective Tissue Conductivity on Thermal Dose Distributions of Living Tissue with Directional Blood Flow During Thermal Therapy”, International Communications in Heat Mass Transfer, 29, 115-126.

Silva R.A., De Lemos M.J.S. and Braga E.J., 2003, "Modeling of Turbulent Natural Convection in Porous Media”, International Communications in Heat Mass Transfer, 30, 615-624.

Silva R. A. and De Lemos M. J. S., 2003, “Turbulent Flow in a Channel Occupied by a Porous Layer Considering the Stress Jump at the Interface”, International Journal of Heat and Mass Transfer, 46, 5113-5121.

Singh A . K., Leonardi E. and Thorpe G. R., 1993, “Three-Dimensional Natural Convection in a Confined Fluid Overlaying a Porous Layer”, ASME Journal of Heat Transfer, 115, 631-638.

Slattey J. C., 1990, “Interfacial Transport Phenomena ”, Springer-Verlang, New York.

Soria A., Whitaker S. and Ochoa-Tapia, J. A., 1997, “The Mass transfer Jump Condition at Boundary Between the Liquid Membrane and the External Regions of Double Emulsion systems”, The First European Congress on Chemical Engineering, Florence, Italy, May 4-7, Proceeding Vol.2, 1295-1298.

Strada, M. and Heinrich J.C., 1982, "Heat Transfer Rates in Natural Convection at High Rayleigh Numbers in Rectangular Enclosures: A Numerical Study” Numerical Heat Transfer, 5, 81-93.

Tan K-K. T., Sam T. and Jamaludin H., 2003, “The onset of Transient Convection in Bottom Heated Porous Media”, International Journal of Heat and Mass Transfer, 46, 28572873.

Tien, C. L. and Hong J.T., 1985, "Natural Convection in Porous Media Under non Darcian and non-uniform Permeability Conditions”, in Natural Convection, S. Kakac et al. eds., Hemisphere, Washington. D.C.

Tong T. W. and Subramanian E., 1986, "Nutural Convection in Rectangular Enclosures Partially Filled with a Porous Medium”, International Journal of Heat and Fluid Flow, 7, (1), 3-10.

Trevisan O. V. and Bejan A., 1985, "Natural Convection with Combined Heat and Mass Transfer Bouyancy Effects in Porous Medium”, International Journal of Heat and Mass Transfer, 28,(8), 1597-1611.

Trevisan O. V. and Bejan A., 1987 "Mass and Heat Transfer by High Rayleigh Number Convection in a Porous Medium Heated from Below", International Journal of Heat and Mass Transfer, 30, 2341-2356.

Vafai K. and Kim S. J., 1990, "Fluid Mechanics of the Interface Region Between a Porous Medium and a Fluid Layer -An Exact Solution”, International Heat Fluid Flow, 11, 254-256.

Vafai K., 2000, “Handbook of Porous Media”, Marcel Dekker Inc., New York. 
Valencia López J.J., 1993, "Modelamiento Matemático de Flujo Convectivo en Almacenamiento de Granos Alimenticios”, Tesis de Maestría, Instituto Tecnológico de Celaya, México.

Wang C., Liao S. and Zhu J., 2003, “An Explicit Solution for the Cobined heat and Mass Transfer by Natural Convection from a Vertical wall in a Non-Darcy Porous Medium”, International Journal of Heat and Mass Transfer, 46, 4813-4822.

Wakashima S. and Saitoh T.S., 2004, "Benchmark Solutions for Natural Convection in a Cubic Cavity Using the High-Order Time-Space Method”, International Journal of Heat and Mass Transfer, 47, 853-864.

Wakitani S., 2001, "Numerical Study of Tree-Dimensional Oscillatory Natural Convection at Low Prandtl Number in Rectangular Enclosures”, Journal of Heat Transfer, 123, 77-83.

Whitaker S., 1966, "The Equation of Motion in Porous Media”, Chemical Engineering Science, 21, 291-300.

Whitaker S., 1967, "Diffusion and Dispersion in Porous Media”, AIChE Journal, 13, 143-183.

Whitaker S., 1969, “Advances in Theory of Fluid Motion in Porous Media”, Flow Through Porous Media Symposium, 61, (12), 14-28.

Whitaker S., 1986a, "Flow in Porous media I: A Theoretical Derivation of Darcy's Law", Transport in Porous Media, 1, 3-25.

Whitaker S., 1986b, "Local Thermal Equilibrium: an application to packed bed catalytic design”, Chemical Engineerinf Science, 41, 2029-20939.

Whitaker S., 1987, "Mass transport and reaction in catalyst pellets", Transport in Porous Media, 22, 269-299.

Whitaker S., 1991, “Improve Constrains for the Principle of Local Thermal Equilibrium”, Ind. \& Eng. Chem., 30, 983-997.

Whitaker S., 1992a, "The Species Mass Jump Condition at a Singular Surface”, Chemical Engineering Science, 47, (7), 1677-1685.

Whitaker S., 1992b, “Improved Constraints for the Principle of Local Thermal Equilibrium”, Ind. \& Eng. Chem., 30, 983-997.

Whitaker S., 1996, “The Forchheimer Equation: A Theoretical Development”, Transport in Porous Media, 25, 27-61

Whitaker S., 1999, “The Methods of Volume Averaging”, Kluwer Academic, Dordrecht, The Netherlands

Woods L. C., 1954, "A Note on the Numerical Solution of Fourth Order Differential Equations”, Aero. Quart., 5, 176-184.

Zanotti F. and Carbonell R. G., 1984a, "Development of Transport Equations for Multiphase Systems I: General Development for Two-Phase Systems”, Chemical Engineering Science, 39, 263-278.

Zanotti F. and Carbonell R. G., 1984b, "Development of Transport Equations for Multiphase Systems II: Application to One-Dimensional Axisymetric flow of Two-Phases”, Chemical Engineering Science, 39, 279-297.

Zanotti F. and Carbonell R. G., 1984c, "Development of Transport Equations for Multiphase Systems III: Application to Heat Transfer in Packed Beds", Chemical Engineering Science, 39, 299-311. 


\section{Apéndice A}

\section{Condición de salto para el transporte de masa en la frontera de un medio poroso y un fluido homogéneo}

Para el modelado de la transferencia de masa en el sistema utilizado en este trabajo se usan ecuaciones de medio efectivo para cada una de las regiones homogéneas o multifásicas. Una opción para obtener estas ecuaciones que gobiernan las concentraciones promedio es el método del promedio volumétrico. Para acoplar estas ecuaciones y analizar los cambios en la interregión se generarán condiciones de salto en la zona fluido-medio poroso (interregión). El objetivo del presente apéndice es el mostrar el desarrollo de la condición de salto para el transporte de un soluto, la forma final de la condición de salto se mostró en el Capítulo 2.

El proceso de transporte de masa en la frontera de un medio poroso y un fluido homogéneo ocurre en una amplia variedad de aplicaciones tecnológicas y por lo tanto ha sido objeto de gran variedad de estudios. Como ejemplos se tienen las columnas de adsorción (Aris, 1959), y el fenómeno de dispersión convectiva que resulta como consecuencia de la transferencia de masa interfacial en cavidades cilíndricas (Lenhoff y Lightfoot, 1984;1986)

Diferentes tipos de condiciones interregionales entre un medio poroso y un fluido fueron analizadas por Alasmi y Vafai (2001). Estos autores mostraron que, en general, las diferencias tienden a mostrar efectos mas pronunciados en los campos de velocidad y efectos substancialmente pequeños en los campos de temperatura y aún más pequeños en la distribución del número de Nusselt.

El procedimiento para obtener la condición de salto para transferencia de masa involucra el desarrollo de una ecuación general promedio, esto es, ella es válida en cualquier parte del sistema fluido-medio poroso. Una vez encontrada esta ecuación se seguirá un procedimiento análogo al establecido para la derivación de la condición de salto interfacial entre fases homogéneas (Slattery, 1990; Whitaker, 1992a). Dicho procedimiento fue propuesto y usado con anterioridad para analizar la transferencia de cantidad de movimiento entre un fluido y un medio poroso Ochoa-Tapia y Whitaker (1995a,b). Posteriormente para la 
transferencia de calor en el mismo sistema Ochoa-Tapia y Whitaker (1998b) y de igual forma para la transferencia de masa en sistemas de emulsiones dobles Ochoa-Tapia y Soria(1995), Soria y col.(1997).

\section{A.1 Ecuaciones puntuales}

Bajo la suposición de solución diluida para un sistema que sólo considera la adsorción, la ecuación gobernante para el transporte del soluto $A$ en el fluido que conforma la fase $\beta$ y que se localiza en ambas regiones $\omega$ y $\eta$ (ver Figura A.1) es:

$$
\frac{\partial C_{A_{\beta}}}{\partial t}+\nabla \cdot\left(C_{A_{\beta}} \mathbf{v}_{\beta}\right)=\nabla \cdot\left(D_{\beta} \nabla C_{A_{\beta}}\right)
$$

La solución de la ecuación (A-1) está sujeta a la siguiente condición de frontera en la interface fluido-sólido:

$$
-\mathbf{n}_{\beta \sigma} \cdot \mathrm{D}_{\beta} \nabla C_{A_{\beta}}=\frac{\partial C_{A_{S}}}{\partial t} \quad \text { en } \mathrm{A}_{\beta \sigma}
$$

Es a través de esta expresión que se consideran el fenómeno de adsorción. Aquí $\mathbf{n}_{\beta \sigma}$ es el vector unitario normal dirigido de la fase $\beta$ a la fase $\sigma$, $\mathrm{A}_{\beta \sigma}$ representa cualquier punto del área interfacial entre el sólido y el fluido, y $C_{A_{s}}$ representa la concentración molar superficial. Esta concentración está relacionada a la del fluido por la siguiente isoterma de adsorción lineal:

$$
C_{A_{s}}=K_{e q} C_{A_{\beta}} \quad \text { en } A_{\beta \sigma}
$$

en donde $K_{e q}$ es el coeficiente en la relación de distribución lineal de equilibrio. Es claro que se está considerando el caso más sencillo posible ya que la isoterma de adsorción es lineal.

De igual forma, la solución de la ecuación (A-1) está sujeta a las condiciones de frontera y a la siguiente condición inicial:
Condición de frontera
$C_{A_{\beta}}=\mathrm{F} \quad(\mathbf{r}, t)$
en $A_{\beta e}$
Condición inicial
$C_{A_{\beta}}=G(\mathbf{r})$
a $\quad t=0$ 


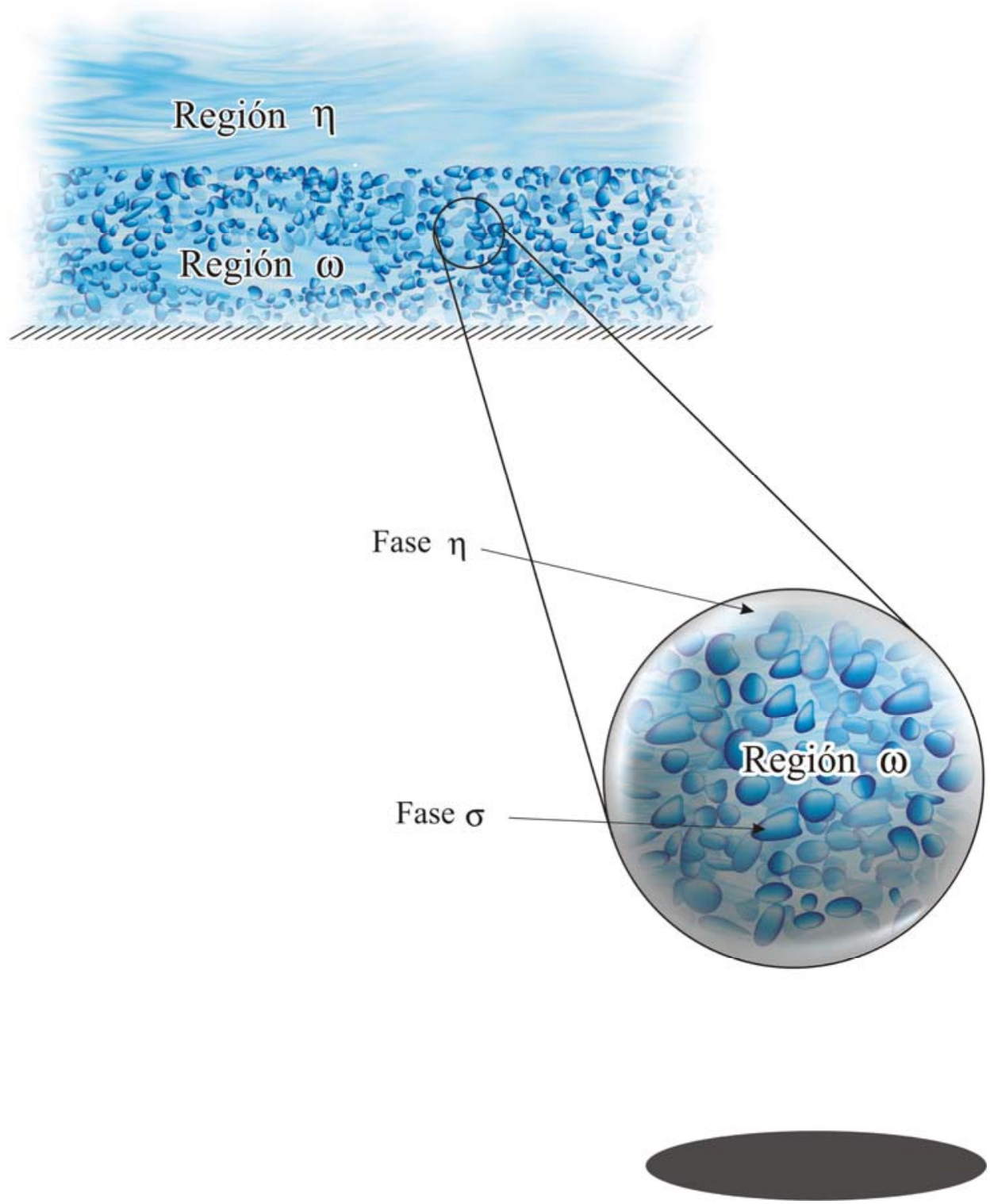

Figura A-1 Sistema considerado. 
En la ecuación (A-4)F $(\mathbf{r}, t)$ representa una funcionalidad en el área de entradas y salidas $A_{\beta e}$ definida por el vector de posición $\mathbf{r}$ en la frontera de la región macroscópica del promedio. Similarmente G (r) representa la funcionalidad de la condición inicial definida en cualquier punto del sistema multifásico.

Adicionalmente a las condiciones dadas por las ecuaciones (A-2) a (A-5), la solución de la ecuación (A-1) requiere conocer el campo de velocidad $\mathbf{v}_{\beta}$ resultante de la solución de las ecuaciones de continuidad

$$
\nabla \cdot \mathbf{v}_{\beta}=0 \quad \text { en la fase } \beta
$$

y Navier-Stokes.

$$
\rho_{\beta}\left(\frac{\partial \mathbf{v}_{\beta}}{\partial t}+\mathbf{v}_{\beta} \cdot \nabla \mathbf{v}_{\beta}\right)=-\nabla p_{\beta}+\rho_{\beta} \mathbf{g}+\mu_{\beta} \nabla^{2} \mathbf{v}_{\beta} \quad \text { en la fase } \beta
$$

El desarrollo de la condición de salto para las ecuaciones de continuidad y de momentum fue realizado por Ochoa-Tapia y Whitaker (1995a,b) y Whitaker (1996). Los resultados obtenidos por esos investigadores permiten la completa definición del problema del movimiento del fluido en términos de las ecuaciones promedio de continuidad y cantidad de movimiento (ecuación de Darcy con la corrección de Brinkman).

Con el propósito tener un modelo factible de solución para el proceso de transferencia de masa en un sistema como el mostrado en la Figura A1, es conveniente obtener las ecuaciones (A-1), (A-2) y (A-3) en su forma volumétricamente promediada en la región $\omega$. Para el seno del lecho poroso, el desarrollo del modelo matemático es relativamente directo ya que las restricciones en las longitudes de escala características establecidas por el método del promedio volumétrico en general son satisfechas facilmente (Zanotti y Carbonell, 1984a,b,c; Carbonell y Whitaker, 1984). Sin embargo, en las vecindades de la interregión dichas restricciones en las longitudes no son satisfechas (ver Figura A.2), obligando a buscar otras opciones para acoplar las ecuaciones de transporte de ambas regiones.

\section{A.2 Volumen Promedio.}

El método del promedio volumétrico es una técnica que puede ser usada para derivar de forma rigurosa las ecuaciones del continuo para sistemas multifásicos. Esto significa que las ecuaciones que son válidas dentro de una fase particular pueden ser homogeneizadas espacialmente para obtener ecuaciones que son válidas en cualquier lugar. Por ejemplo en un sistema fluido-sólido, es necesario saber como el fluido es transportado a través de los poros. El análisis directo de este proceso, en términos de ecuaciones de transporte que son válidas 
dentro de los poros, es esencialmente imposible por lo complejo de la estructura del medio poroso. En lugar de atacar este problema en términos de ecuaciones y condiciones de frontera que son válidas en los poros, se hará uso de la información a escala del poro para derivar las ecuaciones en cualquier parte.

En el planteamiento del modelo para la región porosa se requieren ecuaciones homogeneizadas espacialmente, tomando las ecuaciones que son válidas dentro de una fase particular y de esta manera obtener ecuaciones válidas en cualquier punto, la técnica que se utiliza para desarrollar de manera rigurosa estas ecuaciones es conocida como el método del promedio volumétrico.

Para el sistema bifásico bajo consideración el volumen promedio $\mathrm{V}$ (ver Figura A-2) puede contener las dos fases, y esto se indica mediante:

$$
\mathrm{V}=V_{\beta}(\mathbf{x})+V_{\sigma}(\mathbf{x})
$$

En donde $V_{\beta}$ y $V_{\sigma}$ representan el volumen ocupado por la fase $\beta$ y $\sigma$ dentro del volumen promedio. En términos de estos volúmenes la porosidad o fracción hueca está definida por:

$$
\varepsilon_{\beta}(\mathbf{x})=\frac{V_{\beta}(\mathbf{x})}{\mathrm{V}}
$$

En la Figura A-2, se muestra que esta propiedad promedio del medio poroso, formado por las fases fluida y sólida es función de la posición, aún si el medio poroso es homogéneo.

Para el proceso de promediado se usarán los promedios superficial e intrínseco definidos por:

Concentración promedio superficial $\quad\left\langle C_{A_{\beta}}\right\rangle=\frac{1}{V} \int_{V_{\beta}(x)} C_{A_{\beta}}\left(x+y_{\beta}\right) d V_{y}$

Concentración promedio intrínseca $\quad\left\langle C_{A_{\beta}}\right\rangle^{\beta}=\frac{1}{V_{\beta}} \int_{V_{\beta}} C_{A_{\beta}} d V$ 


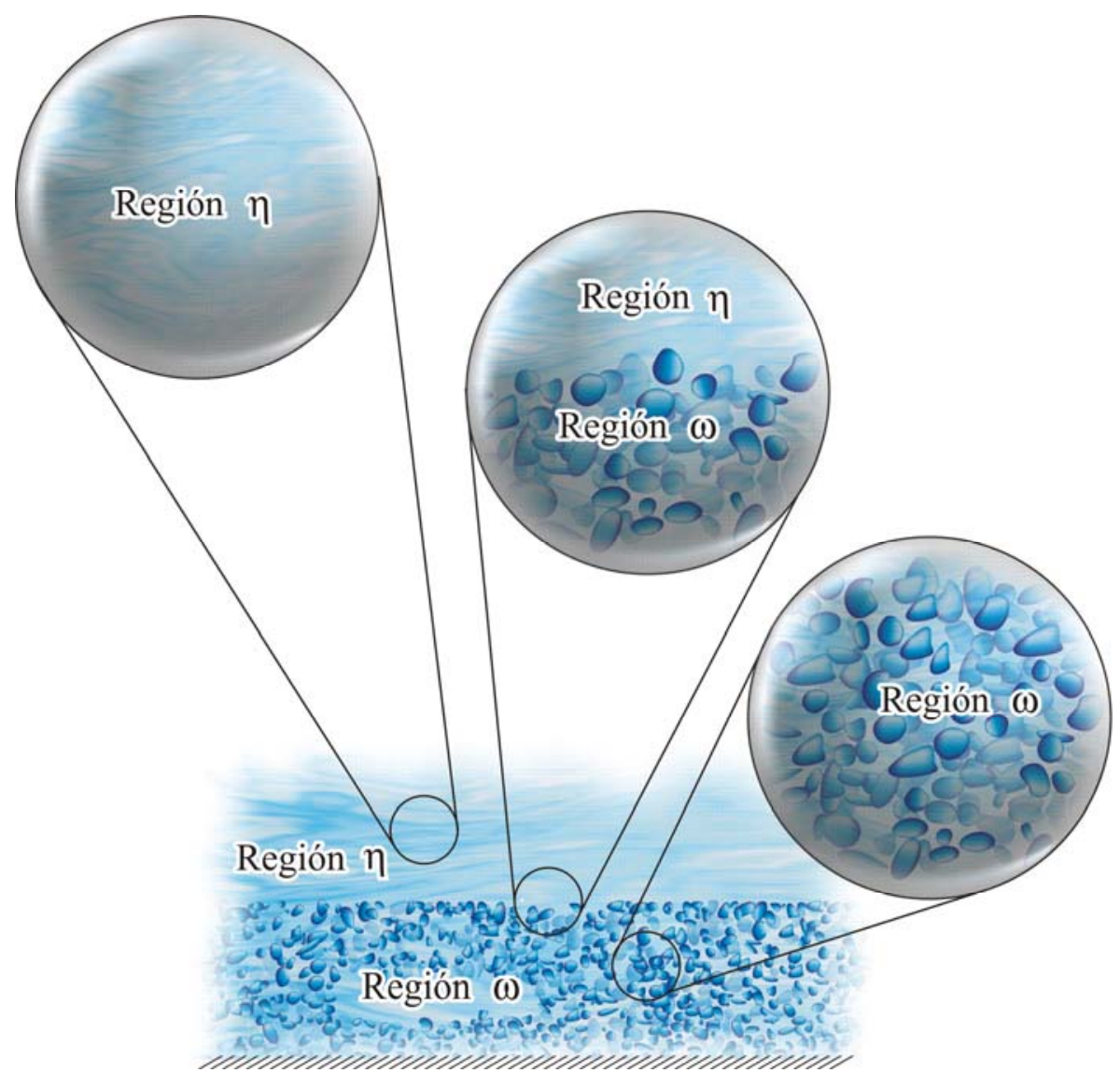

Figura A-2. Posible ubicación del volumen promedio que muestra que la fracción volumétrica en función de la posición del volumen promedio. 
En el presente estudio se desarrollará la ecuación volumétricamente promediada de transferencia de masa válida en cualquier parte del sistema ilustrado en la Figura A-1. Este procedimiento lo iniciamos al reconocer que el volumen promedio puede estar localizado en cualquier parte del espacio en consideración. Hay tres posibilidades claramente diferentes y ellas son mostradas en la Figura A-2. Los detalles del volumen promedio y los vectores involucrados que definen su posición y puntos dentro de él son presentados en la Figura A-3. En ésta se incluyen el vector de posición $\mathbf{r}$ que localiza cualquier punto en el espacio tridimensional $\mathbf{R}^{3}$, el vector de posición $\mathbf{x}$ que localiza el centroide del volumen promedio, el cual puede ubicarse tanto en la fase fluida $(\beta)$ como en la sólida $(\sigma)$, y el vector de posición $\mathbf{y}_{\beta}$ que es usado para representar puntos en la fase $\beta$ relativos al centroide del volumen promedio.

Los dos tipos de concentraciones promedio están relacionadas por la fracción de huecos mediante:

$$
\left\langle C_{A_{\beta}}\right\rangle=\varepsilon_{\beta}\left\langle C_{A_{\beta}}\right\rangle^{\beta}
$$

Cabe hacer notar que en la frontera de las regiones $\omega$ y $\eta$ la fracción volumétrica $\left(\varepsilon_{\beta}\right)$ experimenta cambios significativos como consecuencia de las variaciones del radio del volumen promedio $r_{0}$.

El procedimiento para la obtención de la ecuación generalizada se comienza al aplicar el operador que define el promedio superficial de la ecuación ecuación (A-1):

$$
\underbrace{\frac{1}{\mathrm{~V}} \int_{V_{\beta}} \frac{\partial C_{A_{\beta}}}{\partial t} d V}_{\text {Acumulación }}+\underbrace{\frac{1}{\mathrm{~V}} \int_{V_{\beta}} \nabla \cdot\left(\mathbf{v}_{\beta} C_{A_{\beta}}\right) d V}_{\text {Convección }}=\underbrace{\frac{1}{\mathrm{~V}} \int_{V_{\beta}} \nabla \cdot\left(\mathrm{D}_{\beta} \nabla C_{A_{\beta}}\right) d V}_{\text {Difusión }}
$$

De ésta, el término de acumulación puede escribirse como

$$
\frac{1}{\mathrm{~V}} \int_{V_{\beta}} \frac{\partial C_{A_{\beta}}}{\partial t} d V=\frac{\partial}{\partial t}\left(\frac{1}{\mathrm{~V}} \int_{V_{\beta}} C_{A_{\beta}} d V\right)=\frac{\partial\left\langle C_{A_{\beta}}\right\rangle}{\partial t}
$$

ya que $V_{\beta}$ es función de la posición, $(\mathbf{x})$, pero no del tiempo. 


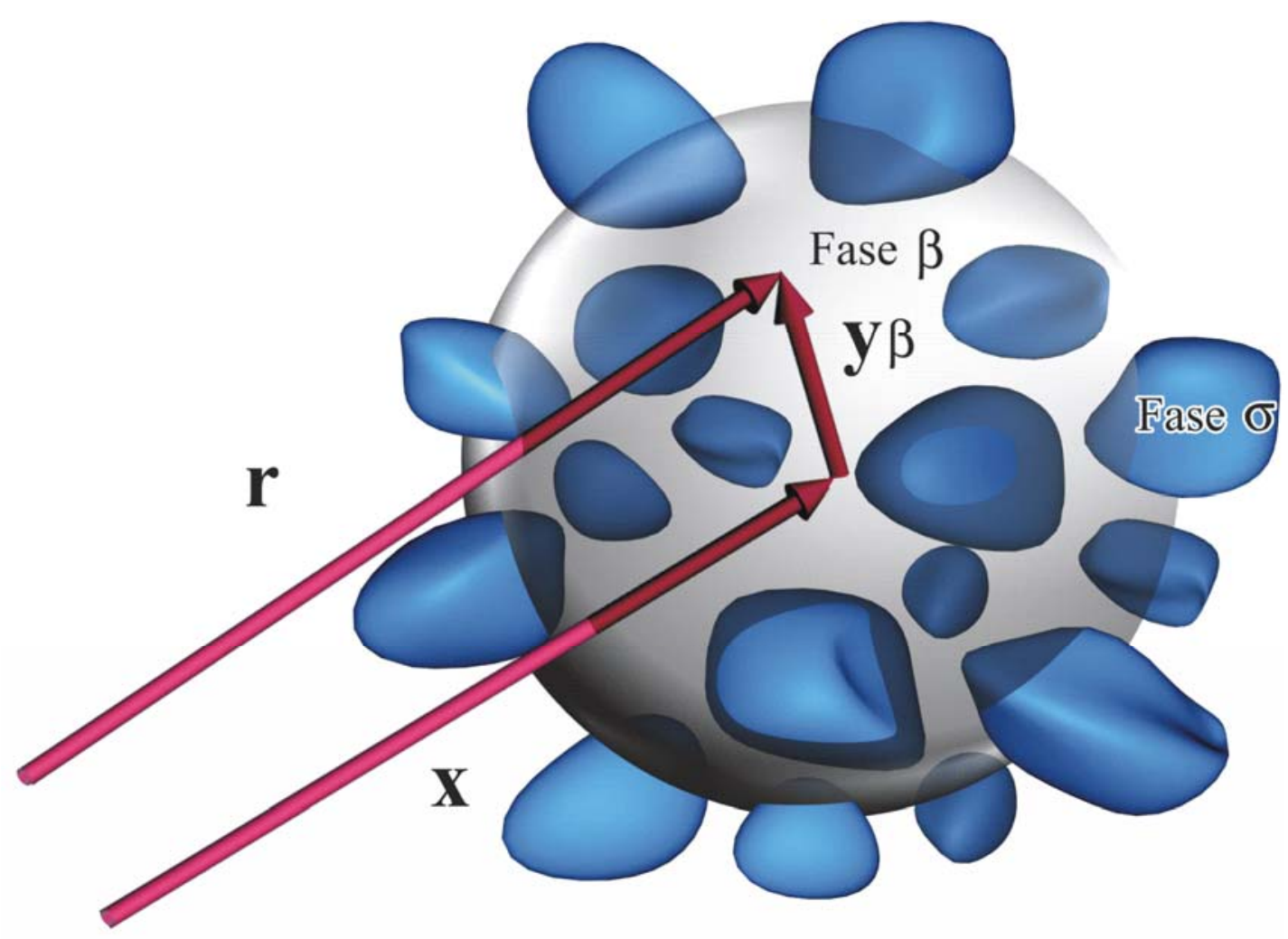

Figura A-3. Volumen promedio y vectores de posición asociados para el sistema fluido-medio poroso. 
Al introducir la relación entre el promedio superficial y el promedio intrínseco, dado por la ecuación (A-10), y restringir el estudio a un medio poroso rígido el término de acumulación toma la forma

$$
\frac{\partial\left\langle C_{A_{\beta}}\right\rangle}{\partial t}=\varepsilon_{\beta} \frac{\partial\left\langle C_{A_{\beta}}\right\rangle^{\beta}}{\partial t}
$$

Por ello la ecuación (A-11) puede escribirse como:

$$
\underbrace{\varepsilon_{\beta} \frac{\partial\left\langle C_{A_{\beta}}\right\rangle^{\beta}}{\partial t}}_{\text {Acumulación }}+\underbrace{\left\langle\nabla \cdot\left(C_{A_{\beta}} \mathbf{v}_{\beta}\right)\right\rangle}_{\text {Convección }}=\underbrace{\left\langle\nabla \cdot\left(D_{\beta} \nabla C_{A_{\beta}}\right)\right\rangle}_{\text {Difusión }}
$$

Con el fin de intercambiar integración y diferenciación en los términos convectivo y difusivo, se hará uso del teorema del promedio volumétrico (Howes y Whitaker, 1985). Este indica que en el sistema de dos fases, $\beta$ y $\sigma$, cualquier variable vectorial $\xi_{\beta}$ asociada con la fase $\beta$, puede expresarse como:

$$
\left\langle\nabla \cdot \xi_{\beta}\right\rangle=\nabla \cdot\left\langle\xi_{\beta}\right\rangle+\frac{1}{V} \int_{A_{\beta \sigma}} \mathbf{n}_{\beta \sigma} \cdot \xi_{\beta} d A
$$

En donde $A_{\beta \sigma}$ representa el área de la interfase $\beta \sigma$ contenida dentro del volumen promedio V (Whitaker, 1967). A continuación se procederá al desarrollo de las ecuaciones promedio de forma separada de los términos convectivo y difusivo.

Desarrollo del término de transporte convectivo

Una primera aplicación del teorema del promedio volumétrico al término convectivo lleva a la siguiente ecuación:

$$
\left\langle\nabla \cdot\left(C_{A_{\beta}} \mathbf{v}_{\beta}\right)\right\rangle=\nabla \cdot\left\langle\mathbf{v}_{\beta} C_{A_{\beta}}\right\rangle+\frac{1}{\mathrm{~V}} \int_{A_{\beta \sigma}} \mathbf{n}_{\beta \sigma} \cdot\left(\mathbf{v}_{\beta} C_{A_{\beta}}\right) d A
$$

Aquí es momento de hacer notar que la fase $\sigma$ es un sólido impermeable, y por ello para la velocidad se tiene:

$$
\mathbf{n}_{\beta \sigma} \cdot \mathbf{v}_{\beta}=0 \quad \text { en la interfase } \beta \sigma
$$


Al aplicar esta condición de frontera en la ecuación (A-16), ella se simplifica a:

$$
\left\langle\nabla \cdot\left(\mathbf{v}_{\beta} C_{A_{\beta}}\right)\right\rangle=\nabla \cdot\left\langle\mathbf{v}_{\beta} C_{A_{\beta}}\right\rangle
$$

La forma tradicional de representar el flux convectivo en términos de desviaciones espaciales (Carbonell y Whitaker, 1983) está dada por:

$$
\left\langle\mathbf{v}_{\beta} C_{A_{\beta}}\right\rangle=\varepsilon_{\beta}\left\langle\mathbf{v}_{\beta}\right\rangle^{\beta}\left\langle C_{A_{\beta}}\right\rangle^{\beta}+\left\langle\tilde{\mathbf{v}}_{\beta} \tilde{C}_{A_{\beta}}\right\rangle
$$

En donde las definiciones para las desviaciones espaciales de velocidad y concentración (Gray, 1975) están dadas por las siguientes expresiones:

$$
\begin{aligned}
& C_{A_{\beta}}=\left\langle C_{A_{\beta}}\right\rangle^{\beta}+\tilde{C}_{A_{\beta}} \\
& \mathbf{v}_{\beta}=\left\langle\mathbf{v}_{\beta}\right\rangle^{\beta}+\tilde{\mathbf{v}}_{\beta}
\end{aligned}
$$

Sin embargo, el uso de la ecuación (A-19) requiere de la imposición de restricciones de longitud de escala (Carbonell y Whitaker, 1984; Quintard y Whitaker, 1994a,b,c,d,e). Para establecer la condición de salto se necesita desarrollar una ecuación que no involucre tales restricciones ya que debe poder usarse en las vecindades de la frontera entre las regiones $\omega$ y $\eta$. Con este propósito se define el flujo dispersivo de exceso, en la misma forma de los trabajos de Ochoa y Whitaker (1997, 1998a), mediante:

$$
\left\langle\mathbf{v}_{\beta} C_{A_{\beta}}\right\rangle_{e x}=\left\langle\mathbf{v}_{\beta} C_{A_{\beta}}\right\rangle-\varepsilon_{\beta}\left\langle\mathbf{v}_{\beta}\right\rangle^{\beta}\left\langle C_{A_{\beta}}\right\rangle^{\beta}-\left\langle\tilde{\mathbf{v}}_{\beta} \tilde{C}_{A_{\beta}}\right\rangle
$$

Con la idea que:

$$
\left\langle\mathbf{v}_{\beta} C_{A \beta}\right\rangle_{e x}=0, \quad \text { en la región homogénea. }
$$

El flujo dispersivo de exceso definido por la ecuación (A-22) no será igual a cero en la frontera $\omega-\eta$, donde las restricciones de longitud de escala desarrolladas por Carbonell y Whitaker (1984) no son válidas.

Entonces, el término convectivo puede expresarse como: 


$$
\nabla \cdot\left\langle\mathbf{v}_{\beta} C_{A_{\beta}}\right\rangle=\underbrace{\nabla \cdot\left(\varepsilon_{\beta}\left\langle\mathbf{v}_{\beta}\right\rangle^{\beta}\left\langle C_{A_{\beta}}\right\rangle^{\beta}\right)}_{\text {Transporte convectivo }}+\underbrace{\nabla \cdot\left\langle\tilde{\mathbf{v}}_{\beta} \tilde{C}_{A_{\beta}}\right\rangle}_{\text {Transporte dispersivo }}+\underbrace{\nabla \cdot\left\langle\mathbf{v}_{\beta} C_{A_{\beta}}\right\rangle_{e x}}_{\text {Dispersión no-local }}
$$

Ahora se procederá al desarrollo detallado del término difusivo.

Desarrollo del término de transporte difusivo

La aplicación del teorema del promedio volumétrico sobre el lado derecho de la ecuación (A-14), lleva al resultado

$$
\left\langle\nabla \cdot\left(\mathrm{D}_{\beta} \nabla C_{A_{\beta}}\right)\right\rangle=\nabla \cdot\left\langle\mathrm{D}_{\beta} \nabla C_{A_{\beta}}\right\rangle+\underbrace{\frac{1}{\mathrm{~V}} \int_{A_{\beta \sigma}} \mathbf{n}_{\beta \sigma} \cdot \mathrm{D}_{\beta} \nabla C_{A_{\beta}} d A}_{\text {Flux interfacial }}
$$

El término integral superficial en esta ecuación puede modificarse, al aplicar la condiciones de frontera interfacial dada por las ecuaciones (A-2) y (A-3), para obtener

$$
\left\langle\nabla \cdot\left(\mathrm{D}_{\beta} \nabla C_{A_{\beta}}\right)\right\rangle=\nabla \cdot\left\langle\mathrm{D}_{\beta} \nabla C_{A_{\beta}}\right\rangle-\frac{1}{\mathrm{~V}} \int_{A_{\beta \sigma}}\left(K_{e q} \frac{\partial C_{A_{\beta}}}{\partial t}\right) d A
$$

En este punto, primero se hace notar que los cambios de la difusividad molecular con la presión, temperatura y concentración, son muy pequeños en longitudes equiparables a la del tamaño del volumen promedio. Así es razonable ignorar las variaciones de este coeficiente molecular de transporte dentro del volumen, V . A continuación, una nueva aplicación del teorema del promedio volumétrico, ahora al primer término del miembro derecho de la ecuación. (A-25), promedio, lleva a:

$$
\left\langle\nabla \cdot\left(\mathrm{D}_{\beta} \nabla C_{A_{\beta}}\right)\right\rangle=\nabla \cdot\left[\mathrm{D}_{\beta}\left(\nabla\left\langle C_{A_{\beta}}\right\rangle+\frac{1}{\mathrm{~V}} \int_{A_{\beta \sigma}} \mathbf{n}_{\beta \sigma} C_{A_{\beta}} d A\right)\right]-\frac{1}{\mathrm{~V}} \int_{A_{\beta \sigma}}\left(K_{e q} \frac{\partial C_{A_{\beta}}}{\partial t}\right) d A
$$

Ahora es conveniente reemplazar la concentración promedio superficial en términos de su promedio intrínseco, y así se obtiene:

$$
\left\langle\nabla \cdot\left(\mathrm{D}_{\beta} \nabla C_{A_{\beta}}\right)\right\rangle=\nabla \cdot\left[\mathrm{D}_{\beta}\left(\varepsilon_{\beta} \nabla\left\langle C_{A_{\beta}}\right\rangle^{\beta}+\left\langle C_{A_{\beta}}\right\rangle^{\beta} \nabla \varepsilon_{\beta}+\frac{1}{\mathrm{~V}} \int_{A_{\beta \sigma}} \mathbf{n}_{\beta \sigma} C_{A_{\beta}} d A\right)\right]
$$




$$
-\frac{1}{\mathrm{~V}} \int_{A_{\beta \sigma}}\left(K_{e q} \frac{\partial C_{A_{\beta}}}{\partial t}\right) d A
$$

Al momento, se ha logrado expresar parte del término derecho de esta ecuación en función de cantidades promedio. No así en los términos que involucran integrales en la superficie interfacial, ya que contienen la concentración puntual. Estos son:
a) $\frac{1}{\mathrm{~V}} \int_{A_{\beta \sigma}} \mathbf{n}_{\beta \sigma} C_{A_{\beta}} d A$
b) $\frac{1}{\mathrm{~V}} \int_{A_{\beta \sigma}}\left(K_{e q} \frac{\partial C_{A_{\beta}}}{\partial t}\right) d A$

Cuando se desarrollan ecuaciones en las que no se toma en cuenta la interregión, el término que considera la concentración puntual se puede desarrollar en series de Taylor, establecer restricciones de escala y poder despreciar los términos de orden superior (Whitaker, 1999). Para evitar esta limitación en el desarrollo, primeramente se hace notar que a partir del teorema del promedio volumétrico se puede establecer que:

$$
\frac{1}{\mathrm{~V}} \int_{A_{\beta \sigma}} \mathbf{n}_{\beta \sigma} d A=-\nabla \varepsilon_{\beta}
$$

La cual es una expresión que no tiene restricciones adicionales, este resultado nos lleva a escribir:

$$
\left.\frac{1}{\mathrm{~V}} \int_{A_{\beta \sigma}} \mathbf{n}_{\beta \sigma}\left\langle C_{A_{\beta}}\right\rangle^{\beta}\right|_{\mathbf{x}} d A=-\left.\left\langle C_{A_{\beta}}\right\rangle^{\beta}\right|_{\mathbf{x}} \nabla \varepsilon_{\beta}=-\left\langle C_{A_{\beta}}\right\rangle^{\beta} \nabla \varepsilon_{\beta}
$$

Es necesario aclarar que las cantidades promedio fuera de una integral son evaluadas en el centroide $\mathbf{x}$ del volumen promedio (Quintard y Whitaker, 1990), pero obviaremos esta notación a menos que se crea indispensable. Usando la ecuación (A-30) en la (A-28) se tiene la siguiente expresión para el término difusivo: 


$$
\begin{array}{r}
\left\langle\nabla \cdot\left(\mathrm{D}_{\beta} \nabla C_{A_{\beta}}\right)\right\rangle=\nabla \cdot\left\{\mathrm{D}_{\beta}[\varepsilon_{\beta} \nabla\left\langle C_{A_{\beta}}\right\rangle^{\beta}+\underbrace{+\frac{1}{\mathrm{~V}} \int_{A_{\beta \sigma}} \mathbf{n}_{\beta \sigma}\left(C_{A_{\beta}}-\left.\left\langle C_{A_{\beta}}\right\rangle^{\beta}\right|_{\mathbf{x}}\right)}_{\text {Difusión no-local }}] d A\right] \\
-\frac{1}{\mathrm{~V}} \int_{A_{\beta \sigma}}\left(K_{e q} \frac{\partial C_{A_{\beta}}}{\partial t}\right) d A
\end{array}
$$

Esta forma de la ecuación para el flux es similar a la obtenida para el de conducción de calor (Nozad y col., 1985; Kaviany 1991; Ochoa y Whitaker 1998). El último término de la ecuación (A-31) puede rescribirse como:

$$
\begin{array}{r}
\left\langle\nabla \cdot\left(\mathrm{D}_{\beta} \nabla C_{A_{\beta}}\right)\right\rangle=\nabla \cdot\left\{\mathrm{D}_{\beta}[\varepsilon_{\beta} \nabla\left\langle C_{A_{\beta}}\right\rangle^{\beta}+\underbrace{\frac{1}{\mathrm{~V}} \int_{A_{\beta \sigma}} \mathbf{n}_{\beta \sigma}\left(C_{A_{\beta}}-\left.\left\langle C_{A_{\beta}}\right\rangle^{\beta}\right|_{\mathbf{x}}\right.}_{\text {Difusión no-local }}] d A\right] \\
-K_{e q} a_{v} \frac{\partial\left\langle C_{A_{\beta}}\right\rangle_{\beta \sigma}}{\partial t}
\end{array}
$$

Aquí se ha supuesto que las variaciones de $K_{e q}$ dentro del volumen promedio son despreciables, y se han introducido las siguientes definiciones:

Concentración promedio interfacial $\quad\left\langle C_{A_{\beta}}\right\rangle_{\beta \sigma}=\frac{1}{A_{\beta \sigma}} \int_{A_{\beta \sigma}} C_{A_{\beta \sigma}} d A$

Área volumétrica interfacial $\quad a_{v}(\mathbf{x})=\frac{A_{\beta \sigma}(\mathbf{x})}{\mathrm{V}}$

Para no expresar la ecuación (A-32) en términos de concentraciones puntuales se hace uso de la definición de desviación espacial para la concentración dada por la ecuación (A-20), y así se llega a: 


$$
\begin{aligned}
& \left\langle\nabla \cdot\left(\mathrm{D}_{\beta} \nabla C_{A_{\beta}}\right)\right\rangle= \\
& \nabla \cdot\left\{\mathrm{D}_{\beta}[\varepsilon_{\beta} \nabla\left\langle C_{A_{\beta}}\right\rangle^{\beta}+\frac{1}{\mathrm{~V}} \int_{A_{\beta \sigma}} \mathbf{n}_{\beta \sigma} \tilde{C}_{A_{\beta}} d A+\underbrace{\left.\frac{1}{\mathrm{~V}} \int_{A_{\beta \sigma}} \mathbf{n}_{\beta \sigma}\left(\left.\left\langle C_{A_{\beta}}\right\rangle^{\beta}\right|_{\mathbf{x}+\mathbf{y}_{\beta}}-\left.\left\langle C_{A_{\beta}}\right\rangle^{\beta}\right|_{\mathbf{x}}\right) d A\right]}_{\text {Difusión no-local }}]\right\} \\
& -K_{e q} a_{v} \frac{\partial\left\langle C_{A_{\beta}}\right\rangle_{\beta \sigma}}{\partial t}
\end{aligned}
$$

Ahora se pueden usar en la ecuación (A-14) los resultados parciales para los términos convectivo y difusivo, ecuaciones (A-23) y (A-35), para obtener:

$$
\begin{aligned}
& \varepsilon_{\beta} \frac{\partial\left\langle C_{A_{\beta}}\right\rangle^{\beta}}{\partial t}+\nabla \cdot\left(\varepsilon_{\beta}\left\langle\mathbf{v}_{\beta}\right\rangle^{\beta}\left\langle C_{A_{\beta}}\right\rangle^{\beta}\right)+\underbrace{\nabla \cdot\left\langle\tilde{\mathbf{v}}_{\beta} \tilde{C}_{A_{\beta}}\right\rangle}_{\text {Dispersión }}+\underbrace{\nabla \cdot\left\langle\mathbf{v}_{\beta} C_{A_{\beta}}\right\rangle_{e x}}_{\text {Dispersión no-local }} \\
& =\nabla \cdot\left\{\mathrm{D}_{\beta}[\varepsilon_{\beta} \nabla\left\langle C_{A_{\beta}}\right\rangle^{\beta}+\frac{1}{\mathrm{~V}} \int_{A_{\beta \sigma}} \mathbf{n}_{\beta \sigma} \tilde{C}_{A_{\beta}} d A+\underbrace{\frac{1}{\mathrm{~V}} \int_{A_{\beta \sigma}} \mathbf{n}_{\beta \sigma}\left(\left.\left\langle C_{A_{\beta}}\right\rangle^{\beta}\right|_{\mathbf{x}+\mathbf{y}_{\beta}}-\left.\left\langle C_{A_{\beta}}\right\rangle^{\beta}\right|_{\mathbf{x}}\right) d A}_{\text {difusión no-local }}]\right\} \\
& -K_{e q} a_{v} \frac{\partial\left\langle C_{A_{\beta}}\right\rangle_{\beta \sigma}}{\partial t}
\end{aligned}
$$

Reordenando la ecuación (A-36) para que tome la forma similar de las ecuaciones de transporte de un soluto en un medio poroso con adsorción, se obtiene

$$
\begin{aligned}
\varepsilon_{\beta}\left(1+\frac{a_{v} K_{e q}}{\varepsilon_{\beta}}\right) \frac{\partial\left\langle C_{A_{\beta}}\right\rangle^{\beta}}{\partial t}+\nabla \cdot\left(\varepsilon_{\beta}\left\langle\mathbf{v}_{\beta}\right\rangle^{\beta}\left\langle C_{A_{\beta}}\right\rangle^{\beta}\right) & =\nabla \cdot\left[\mathbf{D}^{*} \cdot \nabla\left\langle C_{A_{\beta}}\right\rangle^{\beta}\right]-K_{e q} k_{\sigma} a_{v}\left\langle C_{A_{\beta}}\right\rangle^{\beta} \\
& -\underbrace{-K_{e q} a_{v}\left[\frac{\partial\left\langle C_{A_{\beta}}\right\rangle_{\beta \sigma}}{\partial t}-\frac{\partial\left\langle C_{A_{\beta}}\right\rangle^{\beta}}{\partial t}\right]}_{\text {fuente de no-equilibrio }}
\end{aligned}
$$

En la ecuación anterior se ha introducido las siguientes definiciones: 
Tensor de dispersión efectiva total

$$
\mathbf{D}^{*}=\mathbf{D}_{\text {eff }}+\mathbf{D}_{D}
$$

Que involucra al Tensor de difusividad efectiva $\left(\mathbf{D}_{\text {eff }}\right)$

$$
\begin{aligned}
\varepsilon_{\beta} \mathbf{D}_{e f f} \cdot \nabla\left\langle C_{A_{\beta}}\right\rangle=\varepsilon_{\beta} \mathbf{D}_{\beta}\left[\nabla\left\langle C_{A_{\beta}}\right\rangle^{\beta}\right. & +\frac{1}{V} \int_{A_{\beta \sigma}} \mathbf{n}_{\beta \sigma} \tilde{C}_{A_{\beta}} d A \\
& +\underbrace{\frac{1}{V} \int_{A_{\beta \sigma}} \mathbf{n}_{\beta \sigma}\left(\left.\left\langle C_{A_{\beta}}\right\rangle^{\beta}\right|_{\mathbf{x}+\mathbf{y}_{\beta}}-\left.\left\langle C_{A_{\beta}}\right\rangle^{\beta}\right|_{\mathbf{x}}\right)}_{\text {difusion no-local }}] d A]
\end{aligned}
$$

y al Tensor de dispersión

$$
\varepsilon_{\beta} \mathbf{D}_{D} \cdot \nabla\left\langle C_{A_{\beta}}\right\rangle=-\varepsilon_{\beta}\left(\nabla \cdot\left\langle\tilde{\mathbf{v}}_{\beta} \tilde{C}_{A_{\beta}}\right\rangle-\nabla \cdot\left\langle\mathbf{v}_{\beta} C_{A_{\beta}}\right\rangle_{e x}\right)
$$

Fuente de no-equilibrio

$$
\Phi=-K_{e q} a_{v}\left[\frac{\partial\left\langle C_{A_{\beta}}\right\rangle_{\beta \sigma}}{\partial t}-\frac{\partial\left\langle C_{A_{\beta}}\right\rangle^{\beta}}{\partial t}\right]
$$

Nótese que se han introducido en ambos miembros de la ecuación (A-39) el término $a_{v} K_{e q} \frac{\partial\left\langle C_{A_{\beta}}\right\rangle^{\beta}}{\partial t}$ para dar lugar a la contribución denominada fuente de no-equilibrio. Esto porque en el desarrollo de la condición de salto dicha fuente involucra diferencias entre la concentración interfacial promedio y su promedio intrínseco que llevará a un resultado con un significado físico más claro.

Cuando las restricciones de tamaño son satisfechas la ec. (A-37) se reduce al siguiente resultado:

$$
\varepsilon_{\beta \omega}\left(1+\frac{a_{v \omega} K_{e q}}{\varepsilon_{\beta \omega}}\right) \frac{\partial\left\langle C_{A_{\beta}}\right\rangle_{\omega}^{\beta}}{\partial t}+\nabla \cdot\left(\varepsilon_{\beta \omega}\left\langle\mathbf{v}_{\beta}\right\rangle_{\omega}^{\beta}\left\langle C_{A_{\beta}}\right\rangle_{\omega}^{\beta}\right)=\nabla \cdot\left(\varepsilon_{\beta \omega} \mathbf{D}_{\omega}^{*} \cdot \nabla\left\langle C_{A_{\beta}}\right\rangle_{\omega}^{\beta}\right)
$$


En el Apéndice $B$ se presenta el desarrollo en detalle de la ecuación promedio válida en la región homogénea del medio poroso (región $\omega$ ). En esta ecuación el subíndice $\omega$ en $a_{v \omega}$, $\varepsilon_{\beta \omega}$ y $\mathbf{D}_{\omega}^{*}$ indica que los parámetros son los correspondientes a la parte homogénea del medio poroso (región $\omega$ ). En el Apéndice $B$, además de la deducción de la ecuación se presentan las restricciones que las longitudes características deben satisfacer, y se deduce el método para la predicción de los coeficientes de transporte efectivo $\mathbf{D}_{D \omega}$ y $\mathbf{D}_{\text {eff } \omega}$, y por lo tanto $\mathbf{D}_{\omega}^{*}$.

Por otro lado en la parte homogénea del fluido (región $\eta$ )

$$
\frac{\partial\left\langle C_{A_{\beta}}\right\rangle_{\eta}^{\beta}}{\partial t}+\nabla \cdot\left(\left\langle\mathbf{v}_{\beta}\right\rangle_{\eta}^{\beta}\left\langle C_{A_{\beta}}\right\rangle_{\eta}^{\beta}\right)=\nabla \cdot\left(\mathrm{D}_{\beta} \nabla \cdot\left\langle C_{A_{\beta}}\right\rangle_{\eta}^{\beta}\right)
$$

La fracción volumétrica de la fase $\beta\left(\varepsilon_{\beta \eta}\right)$ es igual a uno, esto implica que $a_{v \eta}=0 \mathrm{y}$ el término no-local es nulo, aquí el subíndice $\eta$ indica que la velocidad y la concentración corresponden a la solución de las ecuaciones de transferencia de cantidad de movimiento (Ochoa y Whitaker, 1995) y continuidad del soluto $A$ válidas en el seno del medio poroso. En el Apéndice $B$ también se presentan las restricciones que deben satisfacerse para que la ecuación (A-46) pueda reemplazar a (A-34) sin error significativo. En este punto del análisis contamos con lo necesario para comenzar el desarrollo de la condición de salto que permitirá acoplar las ecuaciones. (A-42) y (A-43).

Los coeficientes efectivos y la fuente de no-equilibrio en la ecuación generalizada (A34), sufrirán variaciones bruscas en la inter-región, lo cual significará una gran complicación en la solución de esa ecuación. Para evitar este problema se usarán las ecuaciones que son válidas estrictamente en las zonas homogéneas de cada una de las regiones. Así, esto significa que los valores obtenidos, $\left\langle C_{A \beta}\right\rangle_{\omega}^{\beta} \mathrm{y}\left\langle C_{A \beta}\right\rangle_{\eta}^{\beta}$, de las ecuaciones (A-42) y (A-43) no serán iguales en la inter-región a los de $\left\langle C_{A \beta}\right\rangle^{\beta}$, obtenidos de la solución de (A-37). Esta idea se muestra esquemáticamente en la Figura A-4 para los casos $\mathbf{n}_{\omega \eta} \cdot \mathbf{D}^{*} \cdot \mathbf{n}_{\omega \eta}>\mathrm{D}_{\beta} \quad \mathrm{y}$ $\mathbf{n}_{\omega \eta} \cdot \mathbf{D}^{*} \cdot \mathbf{n}_{\omega \eta}<\mathrm{D}_{\beta}$. Es importante comprender que los valores de $\left\langle C_{A \beta}\right\rangle_{\omega}^{\beta} \mathrm{y}\left\langle C_{A \beta}\right\rangle_{\eta}^{\beta}$, obtenidos por el procedimiento descrito no serán extrapolaciones, sino soluciones de las ecuaciones que son válidas en las regiones homogéneas $\eta$ y $\omega$ pero que se aplicarán a todos los puntos de la región correspondiente, incluidos los de la inter-región. Debe enfatizarse también que la fracción volumétrica $\varepsilon_{\beta}$ es una función continua de la posición, que toma el valor $\varepsilon_{\beta \omega}$ en la zona homogénea del medio poroso y $\varepsilon_{\beta \eta}$ en la parte homogénea del fluido. Por ello la 
concentración superficial $\left\langle C_{A \beta}\right\rangle$, es también una función continua en cualquier lugar del sistema de dos fases, incluyendo puntos en la inter-región.

Desarrollo de la condición de salto para el flux de A

Para la condición de salto se considera un volumen promedio a gran escala mostrado en la Figura A-5 y recordando que dentro del cual es válida la ecuación de transferencia de masa obtenida en la sección anterior en cualquiera de sus puntos, ya que no se establecieron restricciones de escala en el desarrollo de la ecuación general, está limitada exclusivamente por la forma de las ecuaciones puntuales y por las condiciones de frontera.

Durante el siguiente desarrollo se hace uso de la idea que la ecuación generalizada de transferencia de masa debe de satisfacerse en promedio dentro del volumen macroscópico mostrado en la Figura A-5. Los volúmenes de las regiones $\omega$ y $\eta$ contenidas dentro de $\mathrm{V}_{\infty}$ se designan por $V_{\omega}$ y $V_{\eta}$ respectivamente, de tal forma que:

$$
\mathrm{V}_{\infty}=V_{\omega}+V_{\eta}
$$

Nótese que estos tres volúmenes son independientes del tiempo y por ello la velocidad de desplazamiento de las superficies relacionadas por $\mathrm{A}_{\infty}=A_{\omega}+A_{\eta}$ es nula.

El procedimiento para el desarrollo de la condición de salto asociada con la frontera $\omega-\eta$ incluye los siguientes pasos:

- Integración de la ecuación de transporte generalizada sobre el volumen total $\left(\mathrm{V}_{\infty}\right)$.

Integración de las ecuaciones que son válidas en las regiones homogéneas $\omega$ y $\eta$ sobre sus correspondientes volúmenes $\left(V_{\omega}\right.$ y $\left.V_{\eta}\right)$. La definición de regiones homogéneas $\omega$ y $\eta$ se refiere a las partes que no son afectadas por los cambios rápidos en la estructura cerca de las fronteras físicas del sistema y ello incluye la inter-región. 

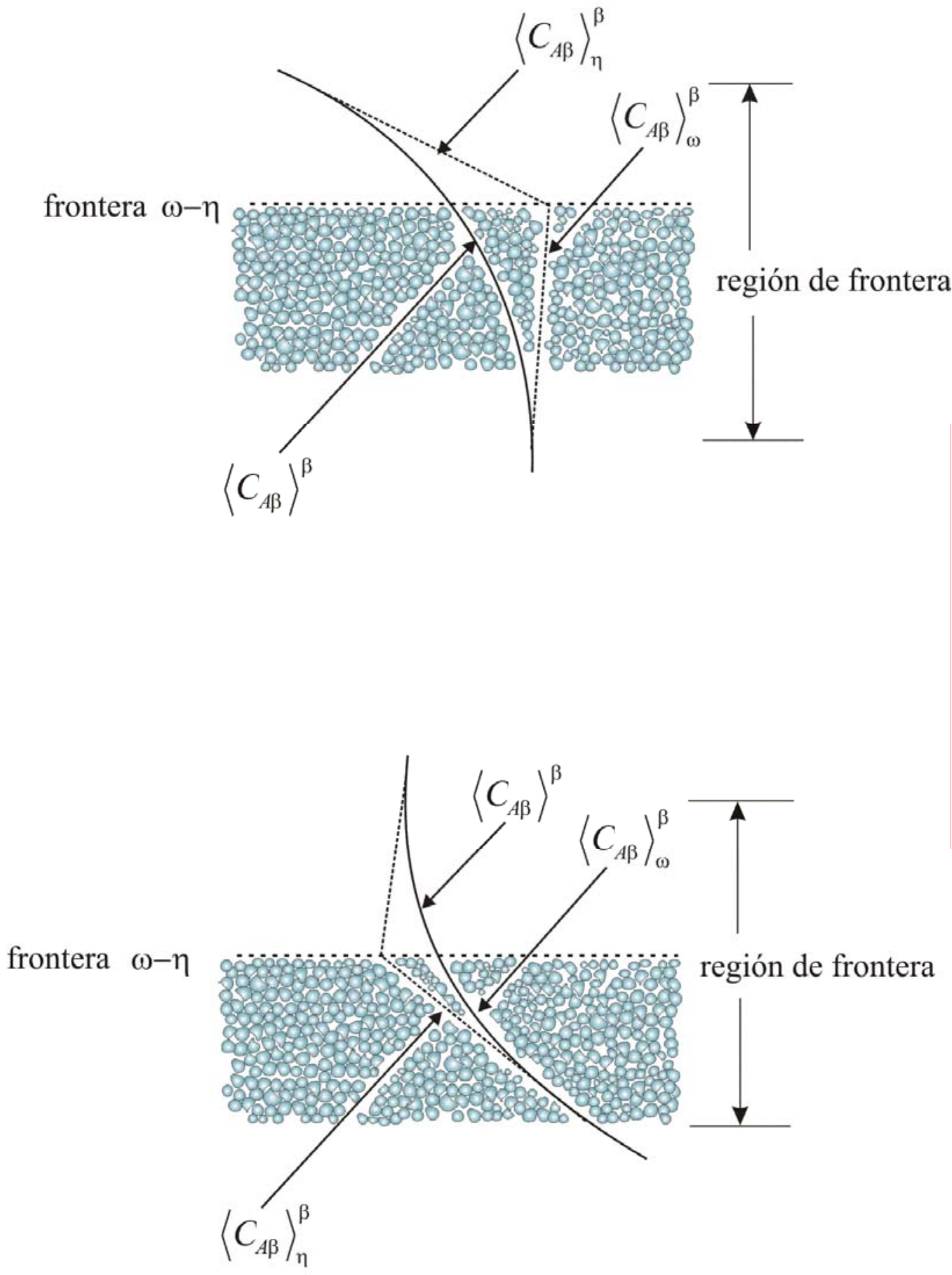

Figura A-4.Continuidad del campo intrínseco y la aproximación en la solución de las ecuaciones válidas en la parte homogénea de las regiones. 
Ahora se procede a realizar tales pasos:

Así se comenzará por la integración de (A-37), en la totalidad del volumen macroscópico, para obtener el resultado:

$$
\begin{gathered}
\int_{V_{\omega}} \varepsilon_{\beta}\left(1+\frac{a_{v} K_{e q}}{\varepsilon_{\beta}}\right) \frac{\partial\left\langle C_{A \beta}\right\rangle^{\beta}}{\partial t} d V+\int_{V_{\eta}} \varepsilon_{\beta}\left(1+\frac{a_{v} K_{e q}}{\varepsilon_{\beta}}\right) \frac{\partial\left\langle C_{A \beta}\right\rangle^{\beta}}{\partial t} d V \\
+\int_{A_{\omega}} \mathbf{n}_{\omega} \cdot\left(\varepsilon_{\beta}\left\langle\mathbf{v}_{\beta}\right\rangle^{\beta}\left\langle C_{A \beta}\right\rangle^{\beta}\right) d A+\int_{A_{\eta}} \mathbf{n}_{\eta} \cdot\left(\varepsilon_{\beta}\left\langle\mathbf{v}_{\beta}\right\rangle^{\beta}\left\langle C_{A \beta}\right\rangle^{\beta}\right) d A \\
=\int_{A_{\omega}} \mathbf{n}_{\omega} \cdot\left(\mathbf{D}^{*} \cdot \nabla\left\langle C_{A \beta}\right\rangle\right) d A+\int_{A_{\eta}} \mathbf{n}_{\eta} \cdot\left(\mathbf{D}^{*} \cdot \nabla\left\langle C_{A \beta}\right\rangle\right) d A \\
-\int_{V_{\omega}} \Phi d V-\int_{V_{\eta}} \Phi d V
\end{gathered}
$$

Para obtener este resultado se ha utilizado el teorema de la divergencia para cambiar varias de las integrales de volumen que originalmente contenían divergencias de flux convectivo y del difusivo. También, se involucró la descomposición de la superficie $A_{\infty}$ en $A_{\omega} \mathrm{y} A_{\eta}$.

La descomposición de la integral mencionada para el flux $\xi_{\beta}$, se expresa como

$$
\int_{V_{\infty}} \nabla \cdot \xi_{\beta} d V=\int_{A_{\omega}} \mathbf{n}_{\omega} \cdot \xi_{\beta} d A+\int_{A_{\eta}} \mathbf{n}_{\eta} \cdot \xi_{\beta} d A
$$

Los vectores unitarios involucrados en las ecuaciones (A-45) y (A-46) se muestran en la Figura A-5. Ahora se procede a integrar las ecuaciones (A-42) y (A-43) en los volúmenes $V_{\omega}$ y $V_{\eta}$ respectivamente. Después de aplicar el teorema de la divergencia en los términos pertinentes se procede a la adición de las dos ecuaciones resultantes para obtener: 


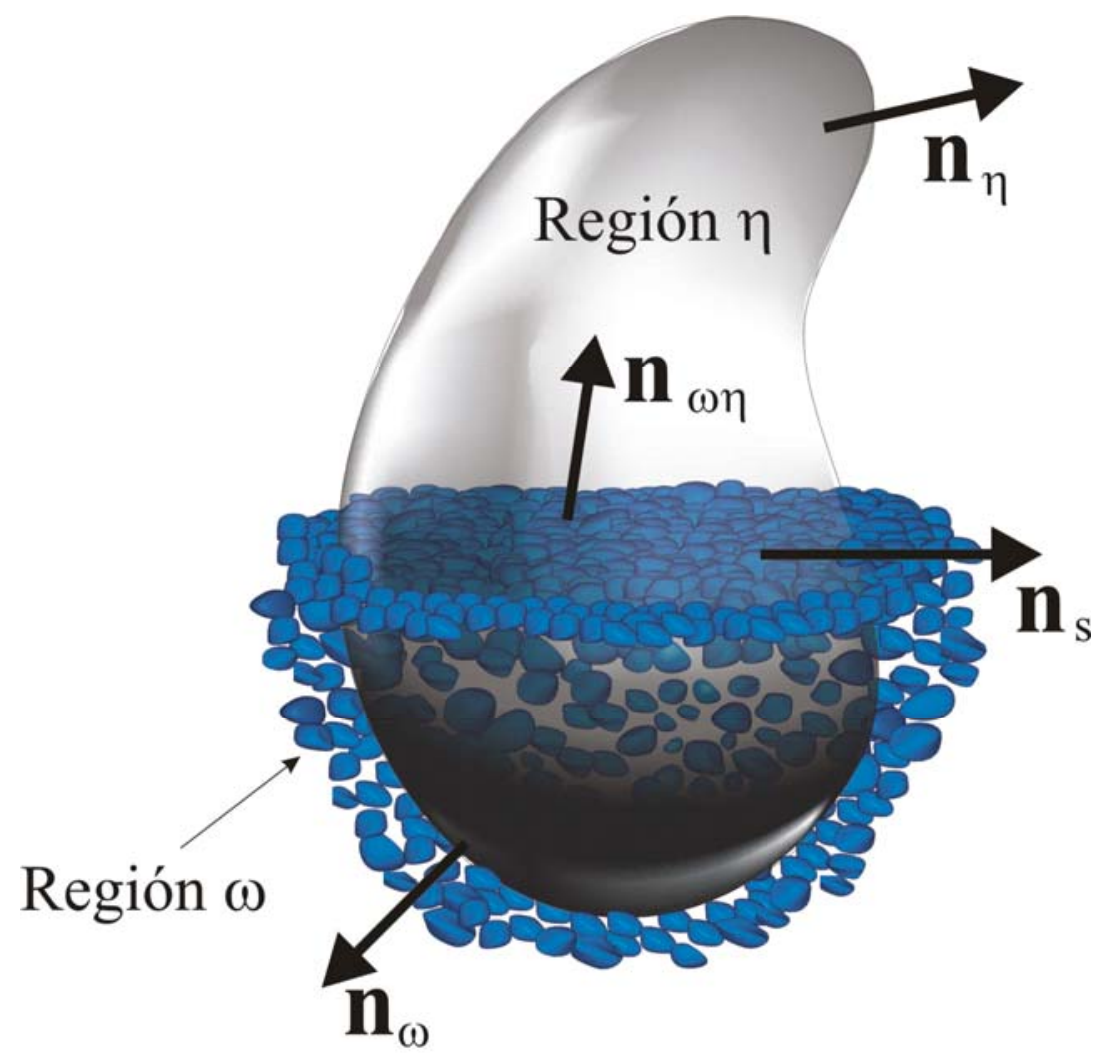

Figura A-5. Volumen promedio a gran escala en la interregión fluido - medio poroso. 


$$
\begin{gathered}
\int_{V_{\omega}} \varepsilon_{\beta \omega}\left(1+\frac{a_{v} K_{e q}}{\varepsilon_{\beta \omega}}\right) \frac{\partial\left\langle C_{A \beta}\right\rangle_{\omega}^{\beta}}{\partial t} d V+\int_{V_{\eta}} \frac{\partial\left\langle C_{A \beta}\right\rangle_{\eta}^{\beta}}{\partial t} d A \\
+\int_{A_{\omega}} \mathbf{n}_{\omega} \cdot\left(\varepsilon_{\beta \omega}\left\langle\mathbf{v}_{\beta}\right\rangle_{\omega}^{\beta}\left\langle C_{A \beta}\right\rangle_{\omega}^{\beta}\right) d A+\int_{A_{\eta}} \mathbf{n}_{\eta} \cdot\left(\left\langle\mathbf{v}_{\beta}\right\rangle_{\eta}^{\beta}\left\langle C_{A \beta}\right\rangle_{\eta}^{\beta}\right) d A \\
+\int_{A_{\omega \eta}} \mathbf{n}_{\omega \eta} \cdot\left(\varepsilon_{\beta \omega}\left\langle\mathbf{v}_{\beta}\right\rangle_{\omega}^{\beta}\left\langle C_{A \beta}\right\rangle_{\omega}^{\beta}-\left\langle\mathbf{v}_{\beta}\right\rangle_{\eta}^{\beta}\left\langle C_{A \beta}\right\rangle_{\eta}^{\beta}\right) d A \\
=\int_{A_{\omega}} \mathbf{n}_{\omega} \cdot\left(\varepsilon_{\beta \omega} \mathbf{D}_{\omega}^{*} \cdot \nabla\left\langle C_{A \beta}\right\rangle_{\omega}^{\beta}\right) d A+\int_{A_{\eta}} \mathbf{n}_{\eta} \cdot\left(D_{\eta} \nabla\left\langle C_{A \beta}\right\rangle_{\eta}^{\beta}\right) d A \\
\quad+\int_{A_{\omega \eta}} \mathbf{n}_{\omega \eta} \cdot\left(\varepsilon_{\beta \omega} \mathbf{D}_{\omega}^{*} \cdot \nabla\left\langle C_{A \beta}\right\rangle_{\omega}^{\beta}-D_{\eta} \nabla\left\langle C_{A \beta}\right\rangle_{\eta}^{\beta}\right) d A
\end{gathered}
$$

En este resultado $A_{\omega \eta}=A_{\eta \omega}$ para representar el área de la superficie que separa las regiones $\omega$ y $\eta$ dentro del volumen $\mathrm{V}_{\infty}$. También se ha utilizado la siguiente nomenclatura para representar el vector unitario en esa superficie, $\mathbf{n}_{\omega \eta}=-\mathbf{n}_{\eta \omega}$.

Restando (A-47) de (A-45) se obtiene:

$$
\begin{gathered}
\int_{V_{\omega}}\left[\varepsilon_{\beta}\left(1+\frac{a_{v} K_{e q}}{\varepsilon_{\beta}}\right) \frac{\partial\left\langle C_{A \beta}\right\rangle^{\beta}}{\partial t}-\varepsilon_{\beta \omega}\left(1+\frac{a_{v} K_{e q}}{\varepsilon_{\beta \omega}}\right) \frac{\partial\left\langle C_{A \beta}\right\rangle_{\omega}^{\beta}}{\partial t}\right] d V \\
+\int_{V_{\eta}}\left[\varepsilon_{\beta}\left(1+\frac{a_{v} K_{e q}}{\varepsilon_{\beta}}\right) \frac{\partial\left\langle C_{A \beta}\right\rangle^{\beta}}{\partial t}-\frac{\partial\left\langle C_{A \beta}\right\rangle_{\eta}^{\beta}}{\partial t}\right] d V \\
+\int_{A_{\omega}} \mathbf{n}_{\omega} \cdot\left(\varepsilon_{\beta}\left\langle\mathbf{v}_{\beta}\right\rangle^{\beta}\left\langle C_{A \beta}\right\rangle^{\beta}-\varepsilon_{\beta \omega}\left\langle\mathbf{v}_{\beta}\right\rangle_{\omega}^{\beta}\left\langle C_{A \beta}\right\rangle_{\omega}^{\beta}\right) d A \\
+\int_{A_{\eta}} \mathbf{n}_{\eta} \cdot\left(\varepsilon_{\beta}\left\langle\mathbf{v}_{\beta}\right\rangle^{\beta}\left\langle C_{A \beta}\right\rangle^{\beta}-\left\langle\mathbf{v}_{\beta}\right\rangle_{\eta}^{\beta}\left\langle C_{A \beta}\right\rangle_{\eta}^{\beta}\right) d A \\
-\int_{A_{\omega \eta}} \mathbf{n}_{\omega \eta} \cdot\left(\varepsilon_{\beta \omega}\left\langle\mathbf{v}_{\beta}\right\rangle_{\omega}^{\beta}\left\langle C_{A \beta}\right\rangle_{\omega}^{\beta}-\left\langle\mathbf{v}_{\beta}\right\rangle_{\eta}^{\beta}\left\langle C_{A \beta}\right\rangle_{\eta}^{\beta}\right) d A \\
=\int_{A_{\omega}} \mathbf{n}_{\omega} \cdot\left(\mathbf{D}^{*} \cdot \nabla\left\langle C_{A \beta}\right\rangle-\varepsilon_{\beta \omega} \mathbf{D}_{\omega}^{*} \cdot \nabla\left\langle C_{A \beta}\right\rangle_{\omega}^{\beta}\right) d A \\
+\int_{A_{\eta}} \mathbf{n}_{\eta} \cdot\left(\mathbf{D}^{*} \cdot \nabla\left\langle C_{A \beta}\right\rangle-\mathrm{D}_{\eta} \nabla\left\langle C_{A \beta}\right\rangle_{\eta}^{\beta}\right) d A \\
-\int_{A_{\omega \eta}} \mathbf{n}_{\omega \eta} \cdot\left(\varepsilon_{\beta \omega} \mathbf{D}_{\omega}^{*} \cdot \nabla\left\langle C_{A \beta}\right\rangle_{\omega}^{\beta}-\mathrm{D}_{\eta} \nabla\left\langle C_{A \beta}\right\rangle_{\eta}^{\beta}\right) d A \\
-\int_{V_{\omega}} \Phi d V-\int_{V_{\eta}} \Phi d V
\end{gathered}
$$


El tipo de términos contenidos en las integrales en las superficies $A_{\omega}$ y $A_{\eta}$ es la característica que Gibbs (1928) definió como una función de exceso en la interfase, una explicación que es aplicable al problema bajo investigación es dado por Ochoa-Tapia y Whitaker 1995a . Introduciendo las definiciones de exceso como:

Acumulación superficial de exceso

$$
\begin{aligned}
\underbrace{\underbrace{}_{A_{\text {ol }}} \varepsilon_{\beta s} \frac{\partial\left\langle C_{A_{\beta}}\right\rangle_{s}^{\beta}}{\partial t} d A}_{\text {Acumulación superficial de exceso }}=\int_{V_{\omega}}\left[\varepsilon_{\beta}\left(1+\frac{a_{v} K_{e q}}{\varepsilon_{\beta}}\right) \frac{\partial\left\langle C_{A_{\beta}}\right\rangle^{\beta}}{\partial t}-\varepsilon_{\beta \omega}\left(1+\frac{a_{v} K_{e q}}{\varepsilon_{\beta \omega}}\right) \frac{\partial\left\langle C_{A_{\beta}}\right\rangle_{\omega}^{\beta}}{\partial t}\right] d V \\
+\int_{V_{\eta}}\left[\varepsilon_{\beta}\left(1+\frac{a_{v} K_{e q}}{\varepsilon_{\beta}}\right) \frac{\partial\left\langle C_{A_{\beta}}\right\rangle^{\beta}}{\partial t}-\frac{\partial\left\langle C_{A_{\beta}}\right\rangle_{\eta}^{\beta}}{\partial t}\right] d V
\end{aligned}
$$

Donde $\varepsilon_{\beta s}$ representa la porosidad superficial de exceso y $\left\langle C_{A_{\beta}}\right\rangle_{S}$ es la concentración superficial.

Transporte convectivo superficial de exceso

$$
\begin{aligned}
& \oint_{C} \mathbf{n}_{s} \cdot \varepsilon_{\beta s}\left\langle\mathbf{v}_{\beta}\right\rangle_{s}^{\beta}\left\langle C_{A \beta}\right\rangle_{s}^{\beta} d \sigma \\
&=\int_{A_{\omega}} \mathbf{n}_{\omega} \cdot\left(\varepsilon_{\beta}\left\langle\mathbf{v}_{\beta}\right\rangle^{\beta}\left\langle C_{A \beta}\right\rangle^{\beta}-\varepsilon_{\beta \omega}\left\langle\mathbf{v}_{\beta}\right\rangle_{\omega}^{\beta}\left\langle C_{A \beta}\right\rangle_{\omega}^{\beta}\right) d A \\
& \quad+\int_{A_{\eta}} \mathbf{n}_{\eta} \cdot\left(\varepsilon_{\beta}\left\langle\mathbf{v}_{\beta}\right\rangle^{\beta}\left\langle C_{A \beta}\right\rangle^{\beta}-\left\langle\mathbf{v}_{\beta}\right\rangle_{\eta}^{\beta}\left\langle C_{A \beta}\right\rangle_{\eta}^{\beta}\right) d A
\end{aligned}
$$

En esta ecuación como puede verse en la Figura A-5, $C$ representa una curva cerrada a lo largo de la línea divide la superficie $A_{\omega \eta}$, mientras $\mathbf{n}_{s}$ representa el vector unitario que es tangente a la frontera $\omega-\eta$ y normal a la curva $C$, y $\sigma$ representa la longitud del arco a lo largo de esta curva. La velocidad superficial es representada por $\varepsilon_{\beta s}\left\langle v_{\beta}\right\rangle_{s}^{\beta}$ (Ochoa-Tapia y Whitaker, 1995a) 
Transporte difusivo superficial de exceso

$$
\begin{aligned}
\oint_{C} \mathbf{n}_{s} \cdot \varepsilon_{\beta s} \mathbf{D}_{s}^{*} \cdot \nabla_{s}\left\langle C_{A \beta}\right\rangle_{s}^{\beta} d \sigma & \\
& =\int_{A_{\omega}} \mathbf{n}_{\omega} \cdot\left(\mathbf{D}^{*} \cdot \nabla\left\langle C_{A \beta}\right\rangle-\varepsilon_{\beta \omega} \mathbf{D}_{\omega}^{*} \cdot \nabla\left\langle C_{A \beta}\right\rangle_{\omega}^{\beta}\right) d A \\
& +\int_{A_{\eta}} \mathbf{n}_{\eta} \cdot\left(\mathbf{D}^{*} \cdot \nabla\left\langle C_{A \beta}\right\rangle-\mathrm{D}_{\eta} \nabla\left\langle C_{A \beta}\right\rangle_{\eta}^{\beta}\right) d A
\end{aligned}
$$

Aquí se uso $\nabla_{s}$ para representar el operador gradiente superficial y está asociado con el operador nabla por $\nabla_{s}=\left(\mathbf{I}-\mathbf{n}_{\omega \eta} \mathbf{n}_{\eta \omega}\right) \cdot \nabla$ (Ochoa-Tapia y col., 1993)

No-equilibrio superficial de exceso

$$
\int_{A_{\omega \eta}} \Phi_{s} d A=\int_{V_{\omega}} \Phi d V+\int_{V_{\eta}} \Phi d V
$$

En donde $\Phi$ queda definido por la ecuación (A-41). Esta definición de acuerdo con trabajos previos (Ochoa-Tapia y Whitaker, 1998b) sugiere que el término de no-equilibrio superficial de exceso puede ser representado como:

$$
\Phi_{s}=\delta \Phi
$$

Donde $\delta$ representa el espesor asociado con la región de frontera y puede ser estimada como la raíz de la norma del tensor permeabilidad en la ley de Darcy.

Substituyendo las ecuaciones. (A-49)-(A-52) en la ecuación (A-48), dando el siguiente resultado:

$$
\begin{aligned}
& \int_{A_{\omega \eta}} \varepsilon_{\beta s} \frac{\partial\left\langle C_{A \beta}\right\rangle_{s}^{\beta}}{\partial t} d A+\oint_{C} \mathbf{n}_{s} \cdot \varepsilon_{\beta s}\left\langle\mathbf{v}_{\beta}\right\rangle_{s}^{\beta}\left\langle C_{A \beta}\right\rangle_{s}^{\beta} d \sigma \\
&-\int_{A_{\omega \eta}} \mathbf{n}_{\omega \eta} \cdot\left(\varepsilon_{\beta \omega}\left\langle\mathbf{v}_{\beta}\right\rangle_{\omega}^{\beta}\left\langle C_{A \beta}\right\rangle_{\omega}^{\beta}-\left\langle\mathbf{v}_{\beta}\right\rangle_{\eta}^{\beta}\left\langle C_{A \beta}\right\rangle_{\eta}^{\beta}\right) d A \\
&=-\int_{A_{\omega \eta}} \mathbf{n}_{\omega \eta} \cdot\left(\varepsilon_{\beta \omega} \mathbf{D}_{\omega}^{*} \cdot \nabla\left\langle C_{A \beta}\right\rangle_{\omega}^{\beta}-D_{\eta} \nabla\left\langle C_{A \beta}\right\rangle_{\eta}^{\beta}\right) d A \\
&+\oint_{C} \mathbf{n}_{s} \cdot \varepsilon_{\beta s} \mathbf{D}_{s}^{*} \cdot \nabla_{s}\left\langle C_{A \beta}\right\rangle_{s}^{\beta} d \sigma-\int_{A_{\omega \eta}} \Phi_{s} d A
\end{aligned}
$$


Usando el teorema de la divergencia superficial (Ochoa-Tapia y col., 1993) para escribir todos los términos bajo la misma área de integración, y entonces el integrando es igual a cero, se obtiene una ecuación de transporte superficial o condición de salto:

$$
\begin{gathered}
\underbrace{\underbrace{\frac{\partial\left\langle C_{A \beta}\right\rangle_{s}^{\beta}}{\partial t}}_{\beta s}+\nabla_{s} \cdot\left[\varepsilon_{\beta S}\left\langle\mathbf{v}_{\beta}\right\rangle_{s}^{\beta}\left\langle C_{A \beta}\right\rangle_{s}^{\beta}-\varepsilon_{\beta S} \mathbf{D}_{s}^{*} \cdot \nabla_{s}\left\langle C_{A \beta}\right\rangle_{s}^{\beta}\right]}_{\text {transporte superficial de exceso }} \\
\underbrace{-\mathbf{n}_{\omega \eta} \cdot\left(\varepsilon_{\beta \omega}\left\langle\mathbf{v}_{\beta}\right\rangle_{\omega}^{\beta}\left\langle C_{A \beta}\right\rangle_{\omega}^{\beta}-\left\langle\mathbf{v}_{\beta}\right\rangle_{\eta}^{\beta}\left\langle C_{A \beta}\right\rangle_{\eta}^{\beta}\right)}_{\text {Transporte convectivo }} \\
=\underbrace{-\mathbf{n}_{\omega \eta} \cdot\left(\varepsilon_{\beta \omega} \mathbf{D}_{\omega}^{*} \cdot \nabla\left\langle C_{A \beta}\right\rangle_{\omega}^{\beta}-D_{\eta} \nabla\left\langle C_{A \beta}\right\rangle_{\eta}^{\beta}\right)}_{\text {transporte difusivo }}-\underbrace{\Phi_{s}}_{\text {no-equilibrio superficial de exceso }}
\end{gathered}
$$

Se debe recordar que hasta este punto no se ha impuesto ninguna condición sobre la concentración promedio en la superficie divisoria del medio poroso y la región fluida. Entonces, basado en la continuidad de los campos $\left\langle C_{A \beta}\right\rangle$ y $\left\langle C_{A \beta}\right\rangle^{\beta}$ mostrados en la Figura A-4, se elige la imposición de continuidad del volumen intrínseco de la continuidad en la frontera $\omega-\eta$.

$$
\left\langle C_{A \beta}\right\rangle_{\omega}^{\beta}=\left\langle C_{A \beta}\right\rangle_{\eta}^{\beta}, \quad \text { en la frontera } \omega-\eta
$$

Lo anterior también implica que la concentración superficial debe satisfacer:

$$
\left\langle C_{A \beta}\right\rangle_{s}^{\beta}=\left\langle C_{A \beta}\right\rangle_{\omega}^{\beta}=\left\langle C_{A \beta}\right\rangle_{\eta}^{\beta}, \quad \text { en la frontera } \omega-\eta
$$

La ecuación (A-61) junto con la continuidad de la velocidad superficial genera la siguiente ecuación:

$$
\mathbf{n}_{\omega \eta} \cdot\left(\varepsilon_{\beta \omega}\left\langle\mathbf{v}_{\beta}\right\rangle_{\omega}^{\beta}\left\langle C_{A \beta}\right\rangle_{\omega}^{\beta}-\left\langle\mathbf{v}_{\beta}\right\rangle_{\eta}^{\beta}\left\langle C_{A \beta}\right\rangle_{\eta}^{\beta}\right)=0 \quad \text { en la frontera } \omega-\eta
$$

Con lo anterior, la ecuación (A-55) se reduce a:

$$
\begin{aligned}
& \underbrace{\varepsilon_{\beta s} \frac{\partial\left\langle C_{A \beta}\right\rangle_{s}^{\beta}}{\partial t}+\nabla_{s} \cdot\left[\varepsilon_{\beta s}\left\langle\mathbf{v}_{\beta}\right\rangle_{s}^{\beta}\left\langle C_{A \beta}\right\rangle_{s}^{\beta}-\varepsilon_{\beta s} \mathbf{D}_{s}^{*} \cdot \nabla_{s}\left\langle C_{A \beta}\right\rangle_{s}^{\beta}\right]}_{\text {Transporte superficial de exceso }} \\
& =\underbrace{-\mathbf{n}_{\omega \eta} \cdot\left(\varepsilon_{\beta \omega} \mathbf{D}_{\omega}^{*} \cdot \nabla\left\langle C_{A \beta}\right\rangle_{\omega}^{\beta}-\mathbf{D}_{\eta} \nabla\left\langle C_{A \beta}\right\rangle_{\eta}^{\beta}\right)}_{\text {Transporte Difusivo }}-\underbrace{\Phi_{s}}_{\text {no-equilibrio superficial de exceso }}
\end{aligned}
$$

Entonces, si el transporte superficial de exceso y el término de no-equilibrio son despreciables, la condición toma la siguiente forma: 


$$
-\mathbf{n}_{\omega \eta} \cdot\left(\varepsilon_{\beta \omega} \mathbf{D}_{\omega}^{*} \cdot \nabla\left\langle C_{A \beta}\right\rangle_{\omega}^{\beta}-\mathbf{D}_{\eta} \nabla\left\langle C_{A \beta}\right\rangle_{\eta}^{\beta}\right)=0
$$

Aunque ésta es una ecuación simplificada, se debe de recordar que debe de ser utilizada junto con las condiciones de velocidad y concentración.

Forma general de la condición de salto

A continuación se seguirán las ideas propuestas por Ochoa-Tapia y Whitaker (1995b) para el estudio del trasporte de cantidad de movimiento. Con base en las definiciones de los términos superficiales de exceso dados por las ecuaciones (A-49)-(A-52) se proponen las siguientes representaciones.

\section{Acumulación superficial de exceso}

$$
\varepsilon_{\beta s} \frac{\partial\left\langle C_{A \beta}\right\rangle_{s}^{\beta}}{\partial t}=\gamma_{a} \varepsilon_{\beta} \frac{\partial\left\langle C_{A \beta}\right\rangle_{\omega}^{\beta}}{\partial t}
$$

\section{Transporte difusivo superficial de exceso}

$$
\nabla_{s} \cdot\left(\varepsilon_{\beta s} \mathbf{D}_{s}^{*} \cdot \nabla_{s}\left\langle C_{A \beta}\right\rangle_{s}^{\beta}\right)=\gamma_{d} \mathbf{n}_{\omega \eta} \cdot\left(\varepsilon_{\beta \omega} \mathbf{D}_{\omega}^{*} \cdot \nabla\left\langle C_{A \beta}\right\rangle_{\omega}^{\beta}-D_{\eta} \nabla\left\langle C_{A \beta}\right\rangle_{\eta}^{\beta}\right)
$$

\section{No-equilibrio superficial de exceso}

$$
\Phi_{s}=\gamma_{s} a_{v} K_{e q} \frac{\partial\left\langle C_{A \beta}\right\rangle_{\omega}^{\beta}}{\partial t}
$$

Note que debido a la forma de la ecuación (A-58), no es necesario introducir una ecuación para el transporte convectivo superficial de exceso. En las ecuaciones. (A-61)-(A-63), $\gamma_{a}, \gamma_{d}$ y $\gamma_{s}$ son parámetros adimensionales de orden uno.

Usando las ecuaciones (A-61)-(A-63) en la ecuación (A-59) se tiene:

$$
\begin{gathered}
\underbrace{\gamma_{a} \varepsilon_{\beta} \frac{\partial\left\langle C_{A \beta}\right\rangle_{\omega}^{\beta}}{\partial t}}_{\text {Acumulación superficial de exceso }}+\underbrace{\gamma_{d} \mathbf{n}_{\omega \eta} \cdot\left(\varepsilon_{\beta \omega} \mathbf{D}_{\omega}^{*} \cdot \nabla\left\langle C_{A \beta}\right\rangle_{\omega}^{\beta}-D_{\eta} \nabla\left\langle C_{A \beta}\right\rangle_{\eta}^{\beta}\right)}_{\text {Transporte superficial de exceso }} \\
=-\underbrace{\mathbf{n}_{\omega \eta} \cdot\left(\varepsilon_{\beta \omega} \mathbf{D}_{\omega}^{*} \cdot \nabla\left\langle C_{A \beta}\right\rangle_{\omega}^{\beta}-D_{\eta} \nabla\left\langle C_{A \beta}\right\rangle_{\eta}^{\beta}\right)}_{\text {Transporte difusivo }}-\underbrace{\gamma_{s} a_{v} K_{e q} \frac{\partial\left\langle C_{A \beta}\right\rangle_{\omega}^{\beta}}{\partial t}}_{\text {no-equilibrio superficial de exceso }}
\end{gathered}
$$

La cual se puede escribir en términos de solo un parámetro ajustable

$$
-\mathbf{n}_{\omega \eta} \cdot\left(\varepsilon_{\beta \omega} \mathbf{D}_{\omega}^{*} \cdot \nabla\left\langle C_{A \beta}\right\rangle_{\omega}^{\beta}-D_{\eta} \nabla\left\langle C_{A \beta}\right\rangle_{\eta}^{\beta}\right)=\gamma a_{v} K_{e q} \frac{\partial\left\langle C_{A \beta}\right\rangle_{\omega}^{\beta}}{\partial t}
$$


En esta ecuación el parámetro $\gamma$ involucra los valores adimensionales $\gamma_{a}, \gamma_{d} \mathrm{y} \gamma_{s}$. Es claro que para las condiciones de estado estable la condición se reduce a aquella que considera la suposición de efectos superficiales de exceso despreciables dado por la ecuación (A-60). En este punto finalmente se debe de reconocer que el término de acumulación de exceso en la ecuación (A-65) es importante. 


\section{Apéndice B}

\section{Problema de cerradura para la ecuación de transferencia de masa en un medio poroso.}

En el Apéndice A se mostró el desarrollo de una ecuación para la condición de salto válida en cualquier región del sistema fluido-medio poroso. Parte importante al aplicar el procedimiento consiste en tener en cuenta los cambios de porosidad al acercarse a la interregión, al final, la ecuación general podría reducirse a la ecuación puntual en la región fluida o bien a la ecuación volumétricamente promediada en la región porosa. El objetivo del presente apéndice es mostrar la aplicación tradicional del método del promedio volumétrico cuando el sistema consiste sólo del medio poroso, muchas de las ecuaciones ya se mencionaron al desarrollar la condición de salto interregional, por lo que se omitirán detalles y sólo se realizará referencia a las ecuaciones del Apéndice A, enfocando el desarrollo detallado al problema de cerradura para la ecuación de transferencia de masa en el medio poroso, así como las restricciones de longitud de escala mencionadas con anterioridad en el Apéndice A.

El sistema considerado es la región porosa mostrada en el la Figura B.1, consisten de una fase sólida inmersa en un fluido. Las ecuaciones consideradas para la transferencia de masa en el medio poroso, se presentaron en el Capítulo 2 y el Apéndice A.

$$
\begin{array}{rlrl}
\frac{\partial C_{A_{\beta}}}{\partial t}+\nabla & \cdot\left(C_{A_{\beta}} \mathbf{v}_{\beta}\right)=\nabla \cdot\left(\mathrm{D}_{\beta} \nabla C_{A_{\beta}}\right) & \\
-\mathbf{n}_{\beta \sigma} \cdot \mathrm{D}_{\beta} \nabla C_{A_{\beta}}=\frac{\partial C_{A_{S}}}{\partial t} & & \text { en } \mathrm{A}_{\beta \sigma} \\
C_{A_{s}} & =K_{e q} C_{A_{\beta}} & & \text { en } \mathrm{A}_{\beta \sigma} \\
C_{A_{\beta}} & =\mathrm{F}(\mathbf{r}, t) & & \text { en } \mathrm{A}_{\beta e} \\
C_{A_{\beta}} & =\mathrm{G}(\mathbf{r}) & & \text { a } t=0
\end{array}
$$

Condición de frontera

Condición inicial 


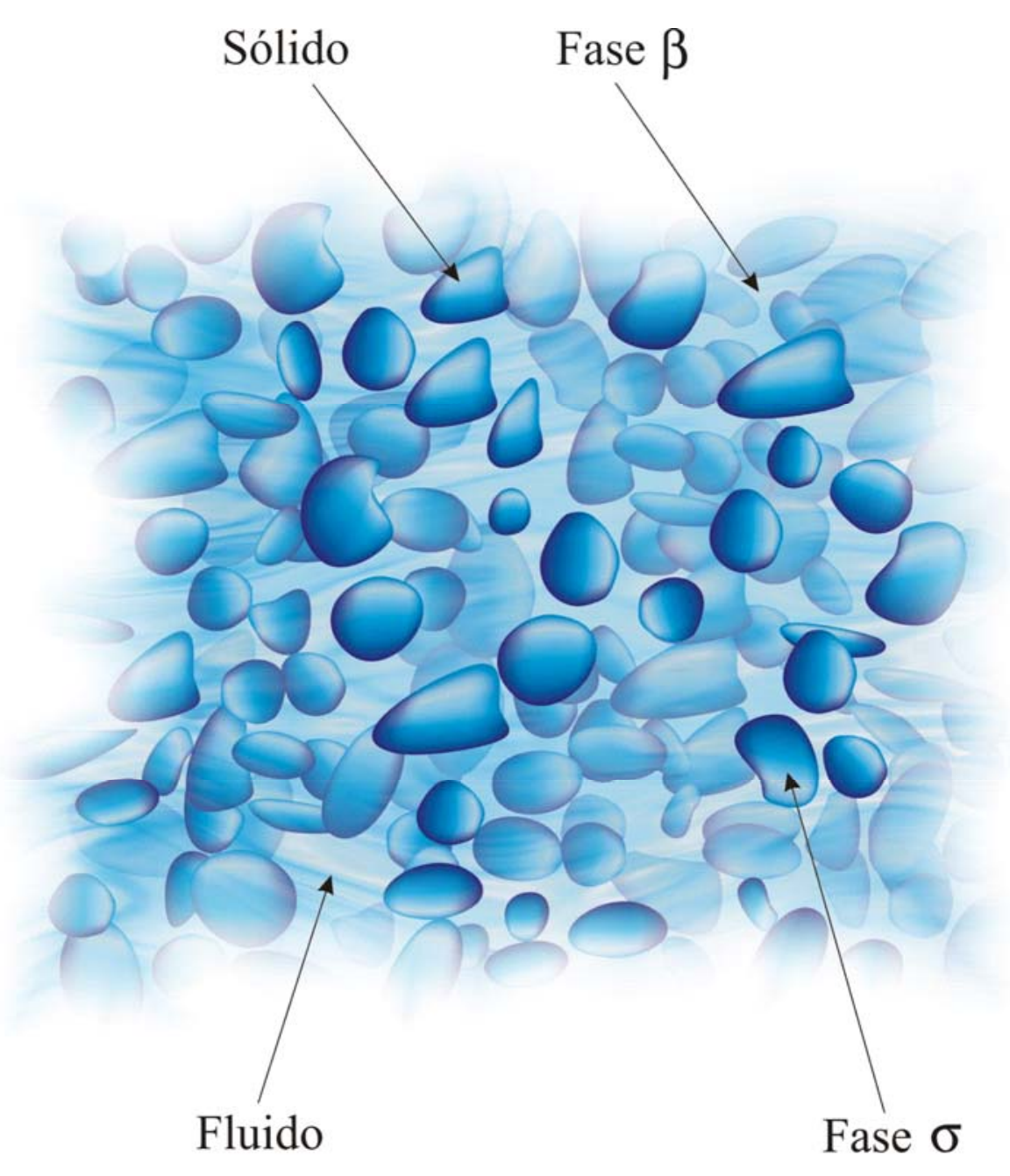

Figura B-1 Transporte convectivo en un sistema poroso (Fluido-solido) 
De acuerdo con el desarrollo presentado en el Apéndice A, para el término convectivo se tiene la siguiente expresión:

$$
\underbrace{\varepsilon_{\beta} \frac{\partial\left\langle C_{A_{\beta}}\right\rangle^{\beta}}{\partial t}}_{\text {Acumulación }}+\underbrace{\left\langle\nabla \cdot\left(C_{A_{\beta}} \mathbf{v}_{\beta}\right)\right\rangle}_{\text {Convección }}=\underbrace{\left\langle\nabla \cdot\left(D_{\beta} \nabla C_{A_{\beta}}\right)\right\rangle}_{\text {Difusión }}
$$

\section{B.1 Transporte convectivo.}

Después de aplicar el promedio volumétrico y la condición de frontera la ecuación para el término convectivo es:

$$
\left\langle\nabla \cdot\left(\mathbf{v}_{\beta} C_{A_{\beta}}\right)\right\rangle=\nabla \cdot\left\langle\mathbf{v}_{\beta} C_{A_{\beta}}\right\rangle
$$

En donde las definiciones para las desviaciones espaciales de velocidad y concentración (Gray, 1975) están dadas por las siguientes expresiones:

$$
\begin{aligned}
& C_{A_{\beta}}=\left\langle C_{A_{\beta}}\right\rangle^{\beta}+\tilde{C}_{A_{\beta}} \\
& \mathbf{v}_{\beta}=\left\langle\mathbf{v}_{\beta}\right\rangle^{\beta}+\tilde{\mathbf{v}}_{\beta}
\end{aligned}
$$

Por lo que el término convectivo expresado en función de desviaciones (Carbonell y Whitaker, 1983) queda expresado mediante:

$$
\left\langle\mathbf{v}_{\beta} C_{A_{\beta}}\right\rangle=\left\langle\left\langle\mathbf{v}_{\beta}\right\rangle^{\beta}\left\langle C_{A_{\beta}}\right\rangle^{\beta}+\tilde{\mathbf{v}}_{\beta}\left\langle C_{A_{\beta}}\right\rangle^{\beta}+\left\langle\mathbf{v}_{\beta}\right\rangle^{\beta} \tilde{C}_{A_{\beta}}+\tilde{\mathbf{v}}_{\beta} \tilde{C}_{A_{\beta}}\right\rangle
$$

En este punto se hace uso de la hipótesis de despreciar las variaciones de las cantidades promedio dentro del promedio, quedando:

$$
\left\langle\mathbf{v}_{\beta} C_{A_{\beta}}\right\rangle=\varepsilon_{\beta}\left\langle\mathbf{v}_{\beta}\right\rangle^{\beta}\left\langle C_{A_{\beta}}\right\rangle^{\beta}+\left\langle\tilde{\mathbf{v}}_{\beta}\right\rangle\left\langle C_{A_{\beta}}\right\rangle^{\beta}+\left\langle\mathbf{v}_{\beta}\right\rangle^{\beta}\left\langle\tilde{C}_{A_{\beta}}\right\rangle+\left\langle\tilde{\mathbf{v}}_{\beta} \tilde{C}_{A_{\beta}}\right\rangle
$$

Utilizando las siguientes definiciones:

$$
\begin{aligned}
& \left\langle\tilde{\mathbf{v}}_{\beta}\right\rangle=0 \\
& \left\langle\tilde{C}_{A_{\beta}}\right\rangle=0
\end{aligned}
$$

De tal manera que la ecuación B-4 se reduce a: 


$$
\left\langle\mathbf{v}_{\beta} C_{A_{\beta}}\right\rangle=\varepsilon_{\beta}\left\langle\mathbf{v}_{\beta}\right\rangle^{\beta}\left\langle C_{A_{\beta}}\right\rangle^{\beta}+\left\langle\tilde{\mathbf{v}}_{\beta} \tilde{\mathrm{C}}_{A_{\beta}}\right\rangle
$$

De donde la ecuación (A-18) adquiere la siguiente forma:

$$
\nabla \cdot\left\langle\mathbf{v}_{\beta} C_{A_{\beta}}\right\rangle=\underbrace{\nabla \cdot\left(\varepsilon_{\beta}\left\langle\mathbf{v}_{\beta}\right\rangle^{\beta}\left\langle C_{A_{\beta}}\right\rangle^{\beta}\right)}_{\text {Transporte Convectivo }}+\underbrace{\nabla \cdot\left\langle\tilde{\mathbf{v}}_{\beta} \tilde{C}_{A_{\beta}}\right\rangle}_{\text {Transporte dispersivo }}
$$

En este punto sólo queda determinar para el termino convectivo, el problema para las desviaciones (problema de cerradura), sin embargo antes analizaremos las restricciones asociadas con la ecuación (B-8).

\section{B.2 Transporte difusivo.}

El desarrollo término difusivo se tomará del Apéndice A hasta la ecuación (A-27), la cual tiene la siguiente forma:

$$
\begin{array}{r}
\left\langle\nabla \cdot\left(\mathrm{D}_{\beta} \nabla C_{A_{\beta}}\right)\right\rangle=\nabla \cdot\left[\mathrm{D}_{\beta}\left(\varepsilon_{\beta} \nabla\left\langle C_{A_{\beta}}\right\rangle^{\beta}+\left\langle C_{A_{\beta}}\right\rangle^{\beta} \nabla \varepsilon_{\beta}+\frac{1}{\mathrm{~V}} \int_{A_{\beta \sigma}} \mathbf{n}_{\beta \sigma} C_{A_{\beta}} d A\right)\right] \\
-\frac{1}{\mathrm{~V}} \int_{A_{\beta \sigma}}\left(K_{e q} \frac{\partial C_{A_{\beta}}}{\partial t}\right) d A
\end{array}
$$

Al momento, se ha logrado expresar parte del término derecho de esta ecuación en función de cantidades promedio. No así en los términos que involucran integrales en la superficie interfacial, ya que contienen la concentración puntual y son:
a) $\frac{1}{\mathrm{~V}} \int_{A_{\beta \sigma}} \mathbf{n}_{\beta \sigma} C_{A_{\beta}} d A$
b) $\frac{1}{\mathrm{~V}} \int_{A_{\beta \sigma}}\left(K_{e q} \frac{\partial C_{A_{\beta}}}{\partial t}\right) d A$

Utilizando la descomposición de la concentración en términos de desviaciones mostrada en la ecuación (B-1) sobre la primera integral y la definición de concentración promedio interfacial expresado por la ecuación (A-33) sobre la segunda integral se tiene:

$\left\langle\nabla \cdot\left(\mathrm{D}_{\beta} \nabla C_{A_{\beta}}\right)\right\rangle=$ 


$$
\begin{array}{r}
\nabla \cdot\left[\mathrm{D}_{\beta}\left(\varepsilon_{\beta} \nabla\left\langle C_{A_{\beta}}\right\rangle^{\beta}+\left\langle C_{A_{\beta}}\right\rangle^{\beta} \nabla \varepsilon_{\beta}+\frac{1}{\mathrm{~V}} \int_{A_{\beta \sigma}} \mathbf{n}_{\beta \sigma}\left\langle C_{A_{\beta}}\right\rangle^{\beta} d A+\frac{1}{\mathrm{~V}} \int_{A_{\beta \sigma}} \mathbf{n}_{\beta \sigma} \tilde{C}_{A_{\beta}} d A\right)\right] \\
-K_{e q} a_{v} \frac{\partial\left\langle C_{A_{\beta}}\right\rangle_{\beta \sigma}}{\partial t}
\end{array}
$$

Whitaker (1999) ha demostrado que la concentración superficial promedio es esencialmente igual a la concentración promedio siempre y cuando se cumplan las siguientes restricciones de escala.

$$
\left(\frac{r_{0}}{L_{C}}\right) \ll 1 \quad\left(\frac{r_{0}^{2}}{L_{C 1} L_{C}}\right) \ll 1
$$

Además que los términos $\left\langle C_{A_{\beta}}\right\rangle^{\beta} \nabla \varepsilon_{\beta} \quad$ y $\frac{1}{\mathrm{~V}} \int_{A_{\beta \sigma}} \mathbf{n}_{\beta \sigma}\left\langle C_{A_{\beta}}\right\rangle^{\beta} d A$ pueden despreciarse cuando se cumplen las siguientes restricciones:

$$
\left(\frac{I_{\beta}}{r_{0}}\right) \ll 1 \quad\left(\frac{r_{0}^{2}}{L_{C 1} L_{\varepsilon}}\right) \ll 1
$$

De tal manera que la ecuación para el término difusivo queda:

$$
\left\langle\nabla \cdot\left(\mathrm{D}_{\beta} \nabla C_{A_{\beta}}\right)\right\rangle=\nabla \cdot\left[\mathrm{D}_{\beta}\left(\varepsilon_{\beta} \nabla\left\langle C_{A_{\beta}}\right\rangle^{\beta}+\frac{1}{\mathrm{~V}} \int_{A_{\beta \sigma}} \mathbf{n}_{\beta \sigma} \tilde{C}_{A_{\beta}} d A\right)\right]-K_{e q} a_{v} \frac{\partial\left\langle C_{A_{\beta}}\right\rangle^{\beta}}{\partial t}
$$

Sustituyendo los términos convectivo (B-8) y difusivo (B-12) en la ecuación (A-14)

$$
\begin{aligned}
\underbrace{\varepsilon_{\beta} \frac{\partial\left\langle C_{A_{\beta}}\right\rangle^{\beta}}{\partial t}}_{\text {Acumulación }}+\underbrace{\nabla \cdot\left(\varepsilon_{\beta}\left\langle\mathbf{v}_{\beta}\right\rangle^{\beta}\left\langle C_{A_{\beta}}\right\rangle^{\beta}\right)}_{\text {Transporte Convectivo }}+\underbrace{\nabla \cdot\left\langle\tilde{\mathbf{v}}_{\beta} \tilde{C}_{A_{\beta}}\right\rangle}_{\text {Transporte dispersivo }} \\
=\nabla \cdot\left[\mathrm{D}_{\beta}\left(\varepsilon_{\beta} \nabla\left\langle C_{A_{\beta}}\right\rangle^{\beta}+\frac{1}{\mathrm{~V}} \int_{A_{\beta \sigma}} \mathbf{n}_{\beta \sigma} \tilde{C}_{A_{\beta}} d A\right]-K_{e q} a_{v} \frac{\partial\left\langle C_{A_{\beta}}\right\rangle^{\beta}}{\partial t}\right.
\end{aligned}
$$

Reagrupando (B-13) 


$$
\begin{aligned}
\left.\varepsilon_{\beta}\left(1+\frac{a_{v} k_{e q}}{\varepsilon_{\beta}}\right) \frac{\partial\left\langle C_{A_{\beta}}\right\rangle^{\beta}}{\partial t}\right|_{\mathbf{x}} & +\underbrace{\nabla \cdot\left(\varepsilon_{\beta}\left\langle\mathbf{v}_{\beta}\right\rangle^{\beta}\left\langle C_{A_{\beta}}\right\rangle^{\beta}\right)}_{\text {Transporte Convectivo }}+\underbrace{\nabla \cdot\left\langle\tilde{\mathbf{v}}_{\beta} \tilde{C}_{A_{\beta}}\right\rangle}_{\text {Transporte dispersivo. }} \\
& =\nabla \cdot\left\{\mathrm{D}_{\beta}\left[\varepsilon_{\beta} \nabla\left\langle C_{A_{\beta}}\right\rangle^{\beta}+\frac{1}{\mathrm{~V}} \int_{A_{\beta \sigma}} \mathbf{n}_{\beta \sigma} \tilde{C}_{A_{\beta}} d A\right]\right\}
\end{aligned}
$$

En este punto sólo queda determinar el problema para las desviaciones (problema de cerradura).

\section{B.3 Problema de cerradura.}

El objetivo del problema de cerradura es encontrar una expresión para la ecuación gobernante de las desviaciones de la concentración, primeramente hay que recordar la definición de las desviaciones:

$$
\tilde{C}_{A_{\beta}}=C_{A_{\beta}}-\left\langle C_{A_{\beta}}\right\rangle^{\beta}
$$

La ecuación puntual esta definida por (A-1) y la ecuación promedio por la (B-12), dividiendo esta última entre $\varepsilon_{\beta}$

$$
\begin{aligned}
\frac{\partial\left\langle C_{A_{\beta}}\right\rangle^{\beta}}{\partial t} & +\frac{1}{\varepsilon_{\beta}} \nabla \cdot\left(\varepsilon_{\beta}\left\langle\mathbf{v}_{\beta}\right\rangle^{\beta}\left\langle C_{A_{\beta}}\right\rangle^{\beta}\right)+\frac{1}{\varepsilon_{\beta}} \nabla \cdot\left\langle\tilde{\mathbf{v}}_{\beta} \tilde{C}_{A_{\beta}}\right\rangle \\
& =\frac{1}{\varepsilon_{\beta}} \nabla \cdot\left[\mathrm{D}_{\beta}\left(\varepsilon_{\beta} \nabla\left\langle C_{A_{\beta}}\right\rangle^{\beta}+\frac{1}{\mathrm{~V}} \int_{A_{\beta \sigma}} \mathbf{n}_{\beta \sigma} \tilde{C}_{A_{\beta}} d A\right)\right]-\frac{a_{v} K_{e q}}{\varepsilon_{\beta}} \frac{\partial\left\langle C_{A_{\beta}}\right\rangle^{\beta}}{\partial t}
\end{aligned}
$$

Donde el término convectivo es:

$$
\frac{1}{\varepsilon_{\beta}} \nabla \cdot\left(\varepsilon_{\beta}\left\langle\mathbf{v}_{\beta}\right\rangle^{\beta}\left\langle C_{A_{\beta}}\right\rangle^{\beta}\right)=\nabla \cdot\left\langle\mathbf{v}_{\beta}\right\rangle^{\beta}\left\langle C_{A_{\beta}}\right\rangle^{\beta}+\frac{1}{\varepsilon_{\beta}}\left\langle\mathbf{v}_{\beta}\right\rangle^{\beta}\left\langle C_{A_{\beta}}\right\rangle^{\beta} \cdot \nabla \varepsilon_{\beta}
$$

y el difusivo

$$
\begin{aligned}
\frac{1}{\varepsilon_{\beta}} \nabla \cdot\left[\mathrm { D } _ { \beta } \left(\varepsilon_{\beta} \nabla\left\langle C_{A_{\beta}}\right\rangle^{\beta}\right.\right. & \left.\left.+\frac{1}{\mathrm{~V}} \int_{A_{\beta \sigma}} \mathbf{n}_{\beta \sigma} \tilde{C}_{A_{\beta}} d A\right)\right]=\nabla \cdot\left(\mathrm{D}_{\beta} \nabla\left\langle C_{A_{\beta}}\right\rangle^{\beta}\right) \\
& +\varepsilon_{\beta}^{-1} \nabla \varepsilon_{\beta} \cdot\left(\mathrm{D}_{\beta} \nabla\left\langle C_{A_{\beta}}\right\rangle^{\beta}\right)+\varepsilon_{\beta}^{-1} \nabla \cdot\left[\frac{\mathrm{D}_{\beta}}{\mathrm{V}} \int_{A_{\beta \sigma}} \mathbf{n}_{\beta \sigma} \tilde{C}_{A_{\beta}} d A\right]
\end{aligned}
$$

Sustituyendo (B-16) y (B-17) en (B-15) se tiene: 


$$
\begin{aligned}
\frac{\partial\left\langle C_{A_{\beta}}\right\rangle^{\beta}}{\partial t}+\nabla \cdot\left(\left\langle\mathbf{v}_{\beta}\right\rangle^{\beta}\left\langle C_{A_{\beta}}\right\rangle^{\beta}\right)+ & \varepsilon_{\beta}^{-1} \nabla \varepsilon_{\beta} \cdot\left\langle\mathbf{v}_{\beta}\right\rangle^{\beta}\left\langle C_{A_{\beta}}\right\rangle^{\beta}=\nabla \cdot\left(\mathrm{D}_{\beta} \nabla\left\langle C_{A_{\beta}}\right\rangle^{\beta}\right) \\
& +\varepsilon_{\beta}^{-1} \nabla \varepsilon_{\beta} \cdot\left(\mathrm{D}_{\beta} \nabla\left\langle C_{A_{\beta}}\right\rangle^{\beta}\right)+\varepsilon_{\beta}^{-1} \nabla \cdot\left[\frac{\mathrm{D}}{\mathrm{V}} \int_{A_{\beta \sigma}} \mathbf{n}_{\beta \sigma} \tilde{C}_{A_{\beta}} d A\right] \\
& -\frac{a_{v} K_{e q}}{\varepsilon_{\beta}} \frac{\partial\left\langle C_{A_{\beta}}\right\rangle^{\beta}}{\partial t}-\varepsilon_{\beta}^{-1} \nabla \cdot\left\langle\tilde{\mathbf{v}}_{\beta} \tilde{C}_{A_{\beta}}\right\rangle
\end{aligned}
$$

De acuerdo con la definición de la ecuación (B-14), la ecuación para las desviaciones se obtienen restando (A-1) de (B-18):

$$
\begin{aligned}
& \frac{\partial \tilde{C}_{A_{\beta}}}{\partial t}+\nabla \cdot\left(\mathbf{v}_{\beta} C_{A_{\beta}}-\left\langle\mathbf{v}_{\beta}\right\rangle^{\beta}\left\langle C_{A_{\beta}}\right\rangle^{\beta}\right)-\varepsilon_{\beta}^{-1} \nabla \varepsilon_{\beta} \cdot\left\langle\mathbf{v}_{\beta}\right\rangle^{\beta}\left\langle C_{A_{\beta}}\right\rangle^{\beta}=\underbrace{\nabla \cdot\left(\mathrm{D}_{\beta} \nabla \tilde{C}_{A_{\beta}}\right)}_{\text {Termino Difusivo }} \\
& -\underbrace{\varepsilon_{\beta}^{-1} \nabla \varepsilon_{\beta} \cdot\left(\mathrm{D}_{\beta} \nabla\left\langle C_{A_{\beta}}\right\rangle^{\beta}\right)}_{\text {Fuente Difusivo }}-\underbrace{\varepsilon_{\beta}^{-1} \nabla \cdot\left[\frac{\mathrm{D}_{\beta}}{\mathrm{V}} \int_{A_{\beta \sigma}} \mathbf{n}_{\beta \sigma} \tilde{C}_{A_{\beta}} d A\right]}_{\text {Termino no local }} \\
& +\underbrace{\frac{a_{v} k_{e q}}{\varepsilon_{\beta}} \frac{\partial\left\langle C_{A_{\beta}}\right\rangle^{\beta}}{\partial t}}_{\text {Fuente Adsortiva }}+\underbrace{\varepsilon_{\beta}^{-1} \nabla \cdot\left\langle\tilde{\mathbf{v}}_{\beta} \tilde{C}_{A_{\beta}}\right\rangle}_{\text {Termino no local }}
\end{aligned}
$$

En la ecuación anterior se tienen dos términos no locales, llamados así porque involucran a la variable dependiente evaluada a lo largo del volumen promedio, no en el centroide. El segundo término en la ecuación (B-19) puede ser reordenado mediante:

$$
\nabla \cdot\left(C_{A_{\beta}} \mathbf{v}_{\beta}-\left\langle C_{A_{\beta}}\right\rangle^{\beta}\left\langle\mathbf{v}_{\beta}\right\rangle^{\beta}\right)=\nabla \cdot\left(\left\langle C_{A_{\beta}}\right\rangle^{\beta} \tilde{\mathbf{v}}_{\beta}\right)+\nabla \cdot\left(\tilde{C}_{A_{\beta}} \mathbf{v}_{\beta}\right)
$$

Desarrollando el lado derecho de la ecuación y aplicando $\nabla \cdot \mathbf{v}_{\beta}=0$

$$
\nabla \cdot\left(C_{A_{\beta}} \mathbf{v}_{\beta}-\left\langle C_{A_{\beta}}\right\rangle^{\beta}\left\langle\mathbf{v}_{\beta}\right\rangle^{\beta}\right)=\left\langle C_{A_{\beta}}\right\rangle^{\beta} \nabla \cdot \tilde{\mathbf{v}}_{\beta}+\tilde{\mathbf{v}}_{\beta} \cdot \nabla\left\langle C_{A_{\beta}}\right\rangle^{\beta}+\mathbf{v}_{\beta} \cdot \nabla \tilde{C}_{A_{\beta}}
$$

De la ecuación de continuidad en términos del promedio intrínseco:

$$
\nabla \cdot \mathbf{v}_{\beta}=\nabla \cdot\left\langle\mathbf{v}_{\beta}\right\rangle^{\beta}+\nabla \cdot \widetilde{\mathbf{v}}_{\beta}=-\varepsilon_{\beta}^{-1}\left\langle\mathbf{v}_{\beta}\right\rangle^{\beta} \cdot \nabla \varepsilon_{\beta}+\nabla \cdot \tilde{\mathbf{v}}_{\beta}=0
$$

Para la desviación de la velocidad. 


$$
\nabla \cdot \widetilde{\mathbf{v}}_{\beta}=\varepsilon_{\beta}^{-1}\left\langle\mathbf{v}_{\beta}\right\rangle^{\beta} \cdot \nabla \varepsilon_{\beta}
$$

Por lo que (B-20) queda:

$$
\nabla \cdot\left(C_{A_{\beta}} \mathbf{v}_{\beta}-\left\langle C_{A_{\beta}}\right\rangle^{\beta}\left\langle\mathbf{v}_{\beta}\right\rangle^{\beta}\right)=\varepsilon_{\beta}^{-1}\left\langle C_{A_{\beta}}\right\rangle^{\beta}\left\langle\mathbf{v}_{\beta}\right\rangle^{\beta} \cdot \nabla \varepsilon_{\beta}+\tilde{\mathbf{v}}_{\beta} \cdot \nabla\left\langle C_{A_{\beta}}\right\rangle^{\beta}+\mathbf{v}_{\beta} \cdot \nabla \tilde{C}_{A_{\beta}}
$$

Sustituyendo (B-24) en (B-19):

$$
\begin{aligned}
& \underbrace{\frac{\partial \tilde{C}_{A_{\beta}}}{\partial t}}_{\text {Acumulación }}+\underbrace{\tilde{\mathbf{v}}_{\beta} \cdot \nabla\left\langle C_{A_{\beta}}\right\rangle^{\beta}}_{\text {Fuente Convectiva }}+\underbrace{\mathbf{v}_{\beta} \cdot \nabla \tilde{C}_{A_{\beta}}}_{\text {Término Convectivo }}=\underbrace{\nabla \cdot\left(\mathrm{D}_{\beta} \nabla \tilde{C}_{A_{\beta}}\right)}_{\text {Término Difusivo }} \\
& -\underbrace{\varepsilon_{\beta}^{-1} \nabla \varepsilon_{\beta} \cdot\left(\mathrm{D}_{\beta} \nabla\left\langle C_{A_{\beta}}\right\rangle^{\beta}\right)}_{\text {Fuente Difusivo }}-\underbrace{\varepsilon_{\beta}^{-1} \nabla \cdot\left[\frac{\mathrm{D}}{\mathrm{V}} \int_{A_{\beta \sigma}} \mathbf{n}_{\beta \sigma} \tilde{C}_{A_{\beta}} d A\right]}_{\text {Termino no local }} \\
& +\underbrace{\frac{a_{v} K_{e q}}{\varepsilon_{\beta}} \frac{\partial\left\langle C_{A_{\beta}}\right\rangle^{\beta}}{\partial t}}_{\text {Fuente Adsortiva }}+\underbrace{\varepsilon_{\beta}^{-1} \nabla \cdot\left\langle\tilde{\mathbf{v}}_{\beta} \tilde{C}_{A_{\beta}}\right\rangle}_{\text {Termino no local }}
\end{aligned}
$$

Igualmente para la condición de frontera (A-2) se usa la definición de desviaciones:

$$
\begin{aligned}
\left(\tilde{C}_{A_{\beta}}=C_{A_{\beta}}-\left\langle C_{A_{\beta}}\right\rangle^{\beta}\right) & : \\
& -\mathbf{n}_{\beta \sigma} \cdot D_{\beta} \nabla\left\langle C_{A_{\beta}}\right\rangle^{\beta}-\mathbf{n}_{\beta \sigma} \cdot \mathrm{D}_{\beta} \nabla \tilde{C}_{A_{\beta}}=k_{e q} \frac{\partial\left\langle C_{A_{\beta}}\right\rangle^{\beta}}{\partial t}+k_{e q} \frac{\partial \tilde{C}_{A_{\beta}}}{\partial t}
\end{aligned}
$$

\section{SIMPLIFICACIONES}

Las ecuaciones (B-25) y (B-26) muestran el problema para las desviaciones con todos los términos involucrados, sin embargo alguno de los términos pueden despreciarse al establecer restricciones de longitud de escala. La primer simplificación se realizará al término de acumulación al limitar el problema de cerradura a la condición de quasi-estado estacionario, esto significa establecer las siguientes restricciones (Whitaker, 1999):

$$
\begin{array}{ll}
\frac{\partial \tilde{C}_{A_{\beta}}}{\partial t}<<\nabla \cdot\left(D_{\beta} \nabla \tilde{C}_{A_{\beta}}\right) & \text { Para la ecuación gobernante } \\
k_{e q} \frac{\partial \tilde{C}_{A_{\beta}}}{\partial t}<<D_{\beta} \nabla \tilde{C}_{A_{\beta}} & \text { Para la condición de frontera }
\end{array}
$$


Realizando una análisis de orden de magnitud para los términos en las ecuaciones (B27) y (B-28) se tiene:

$$
\begin{aligned}
\frac{\partial \tilde{C}_{A_{\beta}}}{\partial t} & =\mathbf{O}\left(\frac{\tilde{C}_{A_{\beta}}}{t^{*}}\right) \\
\nabla \tilde{C}_{A_{\beta}} & =\mathbf{O}\left(\frac{\tilde{C}_{A_{\beta}}}{l_{\beta}}\right) \\
\nabla^{2} \tilde{C}_{A_{\beta}} & =\mathbf{O}\left(\frac{\tilde{C}_{A_{\beta}}}{l_{\beta}^{2}}\right)
\end{aligned}
$$

Por lo que las restricciones de escala para los términos de acumulación son:

$$
\begin{array}{ll}
\frac{\mathrm{D}_{\beta} t^{*}}{l_{\beta}^{2}}>>1 & \text { Para la ecuación gobernante } \\
\frac{\mathrm{D}_{\beta} t^{*}}{k_{\text {eq. }} l_{\beta}}>>1 & \text { Para la condición de frontera }
\end{array}
$$

Con lo anterior el sistema de ecuación gobernante (B-25) y condición de frontera (B-26) quedan:

$$
\begin{aligned}
\underbrace{\tilde{\mathbf{v}}_{\beta} \cdot \nabla\left\langle C_{A_{\beta}}\right\rangle^{\beta}}_{\text {Fuente Convectiva }}+\underbrace{\mathbf{v}_{\beta} \cdot \nabla \tilde{C}_{A_{\beta}}}_{\text {Término Convectivo }} & =\underbrace{\nabla \cdot\left(\mathrm{D}_{\beta} \nabla \tilde{C}_{A_{\beta}}\right)}_{\text {Término Difusivo }} \\
& -\underbrace{\varepsilon_{\beta}^{-1} \nabla \varepsilon_{\beta} \cdot\left(\mathrm{D}_{\beta} \nabla\left\langle C_{A_{\beta}}\right\rangle^{\beta}\right)}_{\text {Fuente Difusiva }}-\underbrace{\varepsilon_{\beta}^{-1} \nabla \cdot\left[\frac{\mathrm{D}_{\beta}}{\mathrm{V}} \int_{A_{\beta \sigma}} \mathbf{n}_{\beta \sigma} \tilde{C}_{A_{\beta}} d A\right]}_{\text {Término no local }} \\
+ & \underbrace{\underbrace{a_{e q}}_{\varepsilon_{\beta}} \frac{\partial\left\langle C_{A_{\beta}}\right\rangle^{\beta}}{\partial t}}_{\text {Fuente Adsortiva }}+\underbrace{\varepsilon_{\beta}^{-1} \nabla \cdot\left\langle\tilde{\mathbf{v}}_{\beta} \tilde{C}_{A_{\beta}}\right\rangle}_{\text {Término no local }} \\
-\mathbf{n}_{\beta \sigma} \cdot \mathrm{D}_{\beta} \nabla \tilde{C}_{A_{\beta}} & =\mathbf{n}_{\beta \sigma} \cdot \mathrm{D}_{\beta} \nabla\left\langle C_{A_{\beta}}\right\rangle^{\beta}+k_{e q} \frac{\partial\left\langle C_{A_{\beta}}\right\rangle^{\beta}}{\partial t} \quad \text { en } A_{\beta \sigma}
\end{aligned}
$$

El siguiente término para analizar es el difusivo no local, para ello primeramente se hace notar el orden de magnitud para la integral:

$$
\left[\frac{1}{\mathrm{~V}} \int_{A_{\beta \sigma}} \mathbf{n}_{\beta \sigma} \tilde{C}_{A_{\beta}} d A\right]=\mathbf{O}\left(a_{v} \tilde{C}_{A_{\beta}}\right)
$$


Y la divergencia como:

$$
\nabla \cdot\left[\frac{1}{\mathrm{~V}} \int_{A_{\beta \sigma}} \mathbf{n}_{\beta \sigma} \tilde{C}_{A_{\beta}} d A\right]=\mathbf{O}\left(\frac{a_{v} \tilde{C}_{A_{\beta}}}{L}\right)
$$

Para la mayoría de los medios porosos, un estimado razonable del orden de magnitud del área por unidad de volumen está dado por:

$$
a_{v}=\mathbf{O}\left(l_{\beta}^{-1}\right)
$$

Con lo anterior el término no local está dado por

$$
\varepsilon_{\beta}^{-1} \nabla \cdot\left[\frac{\mathrm{D}_{\beta}}{\mathrm{V}} \int_{A_{\beta \sigma}} \mathbf{n}_{\beta \sigma} \tilde{C}_{A_{\beta}} d A\right]=\mathrm{O}\left(\frac{\varepsilon_{\beta}^{-1} \mathrm{D}_{\beta} \tilde{C}_{A_{\beta}}}{l_{\beta} L}\right)
$$

Por otro lado el orden de magnitud del término difusivo queda expresado por:

$$
\nabla \cdot\left(\mathrm{D}_{\beta} \nabla \tilde{C}_{A_{\beta}}\right)=\mathbf{O}\left(\frac{D_{\beta} \tilde{C}_{A_{\beta}}}{l_{\beta}^{2}}\right)
$$

Haciendo una comparación del orden de magnitud se puede despreciar el término difusivo no local de acuerdo con:

$$
\varepsilon_{\beta}^{-1} \nabla \cdot\left[\frac{\mathrm{D}_{\beta}}{V} \int_{A_{\beta \sigma}} \mathbf{n}_{\beta \sigma} \tilde{C}_{A_{\beta}} d A\right]<<\nabla \cdot\left(\mathrm{D}_{\beta} \nabla \tilde{C}_{A_{\beta}}\right)
$$

Es fácil demostrar que el término de la fuente difusiva pude ser despreciada en relación con la fuente convectiva, esto es:

$$
\underbrace{\tilde{\mathbf{v}}_{\beta} \cdot \nabla\left\langle C_{A_{\beta}}\right\rangle^{\beta}}_{\text {Fuente Convectiva }} \gg \underbrace{\varepsilon_{\beta}^{-1} \nabla \varepsilon_{\beta} \cdot\left(\mathrm{D}_{\beta} \nabla\left\langle C_{A_{\beta}}\right\rangle^{\beta}\right)}_{\text {Fuente Difusiva }}
$$

Estimando $\widetilde{\mathbf{v}}_{\beta}$ como $\left\langle\mathbf{v}_{\beta}\right\rangle^{\beta}$ ya que la condición de no desplazamiento requiere que $\widetilde{\mathbf{v}}_{\beta} \mathrm{y}$ $\left\langle\mathbf{v}_{\beta}\right\rangle^{\beta}$ sean del mismo orden de magnitud, se tiene la siguiente restricción:

$$
\left\langle\mathbf{v}_{\beta}\right\rangle^{\beta} \gg \varepsilon_{\beta}^{-1} \frac{\varepsilon_{\beta}}{L_{\varepsilon}} D_{\beta}
$$


Multiplicando por $l_{\beta}$ y reagrupando (B-43)

$$
\frac{\left\langle\mathbf{v}_{\beta}\right\rangle^{\beta} l_{\beta}}{D_{\beta}} \gg \frac{l_{\beta}}{L_{\varepsilon}}
$$

Donde $P e=\frac{\left\langle v_{\beta}\right\rangle^{\beta} l_{\beta}}{D_{\beta}}$, por lo que la restricción para despreciar la fuente difusiva es

$$
P e>>\frac{l_{\beta}}{L_{\varepsilon}}
$$

Esta restricción es automáticamente satisfecha en la región homogénea del medio poroso ya $L_{\varepsilon}$ que es infinito.

Al despreciar los términos difusivo no local y la fuente difusiva, la ecuación gobernante para las desviaciones de la concentración es:

$$
\underbrace{\tilde{\mathbf{v}}_{\beta} \cdot \nabla\left\langle C_{A_{\beta}}\right\rangle^{\beta}}_{\text {Fuente Convectiva }}+\underbrace{\mathbf{v}_{\beta} \cdot \nabla \tilde{C}_{A_{\beta}}}_{\text {Término Convectivo }}=\underbrace{\nabla \cdot\left(D_{\beta} \nabla \tilde{C}_{A}\right)}_{\text {Término Difusivo }}+\underbrace{\frac{a_{v} k_{e q}}{\varepsilon_{\beta}} \frac{\partial\left\langle C_{A_{\beta}}\right\rangle^{\beta}}{\partial t}}_{\text {Fuente Adsortiva }}+\underbrace{\varepsilon_{\beta}^{-1} \nabla \cdot\left\langle\tilde{\mathbf{v}}_{\beta} \tilde{C}_{A_{\beta}}\right\rangle}_{\text {Término no local }}
$$

El siguiente término a analizar es el término no local de transporte dispersivo, la comparación con la parte difusiva es:

$$
\varepsilon_{\beta}^{-1} \nabla \cdot\left\langle\tilde{\mathbf{v}}_{\beta} \tilde{C}_{A_{\beta}}\right\rangle \ll \nabla \cdot\left(\mathbf{D}_{\beta} \nabla \tilde{C}_{A_{\beta}}\right)
$$

El orden de magnitud del transporte no local puede ser estimado acorde con:

$$
\varepsilon_{\beta}^{-1} \nabla \cdot\left\langle\tilde{\mathbf{v}}_{\beta} \tilde{C}_{A_{\beta}}\right\rangle=\mathbf{O}\left(\frac{\left\langle\mathbf{v}_{\beta}\right\rangle^{\beta} \tilde{C}_{A_{\beta}}}{L}\right)
$$

En el resultado anterior nuevamente se estimó $\widetilde{\mathbf{v}}_{\beta}$ como $\left\langle\mathbf{v}_{\beta}\right\rangle^{\beta}$, el orden de magnitud de la parte difusiva queda expresado por (B-40) quedando:

$$
\frac{\varepsilon_{\beta}^{-1} \tilde{C}_{A_{\beta}}\left\langle\mathbf{v}_{\beta}\right\rangle^{\beta}}{L} \ll \frac{\mathrm{D}_{\beta} \tilde{C}_{A_{\beta}}}{\delta_{\beta}^{2}}
$$


Donde $\delta_{\beta}$ es la pequeña longitud de escala asociada con $\widetilde{C}_{A}$, cuando el transporte es puramente difusivo se puede concluir que:

$$
\delta_{\beta}=l_{\beta} \quad \text { Proceso difusivo }
$$

Reagrupando (B-49) en términos del Número de Peclet, la restricción para despreciar el término no local de transporte difusivo es:

$$
P e<<\frac{l_{\beta} L}{\delta_{\beta}^{2}}
$$

Al despreciar este ultimo término, la ecuación gobernante y condiciones de frontera para las desviaciones son:

$$
\begin{aligned}
& \underbrace{\tilde{\mathbf{v}}_{\beta} \cdot \nabla\left\langle C_{A_{\beta}}\right\rangle^{\beta}}_{\text {Fuente Convectiva }}+\underbrace{\mathbf{v}_{\beta} \cdot \nabla \tilde{C}_{A_{\beta}}}_{\text {Termino Convectivo }}=\underbrace{\nabla \cdot\left(\mathrm{D}_{\beta} \nabla \tilde{C}_{A_{\beta}}\right)}_{\text {Termino Difusivo }}+\underbrace{\frac{a_{v} k_{e q}}{\varepsilon_{\beta}} \frac{\partial\left\langle C_{A_{\beta}}\right\rangle^{\beta}}{\partial t}}_{\text {Fuente Adsortiva }} \\
& -\mathbf{n}_{\beta \sigma} \cdot \mathrm{D}_{\beta} \nabla \tilde{C}_{A_{\beta}}=\underbrace{\mathbf{n}_{\beta \sigma} \cdot \mathrm{D}_{\beta} \nabla\left\langle C_{A_{\beta}}\right\rangle^{\beta}}_{\text {Fuente Difusiva }}+\underbrace{k_{e q} \frac{\partial\left\langle C_{A_{\beta}}\right\rangle^{\beta}}{\partial t}}_{\text {Fuente Adsortiva }} \\
& C_{A_{\beta}}=\mathrm{F} \quad(\mathbf{r}, \mathrm{t}) \quad \text { en } A_{\beta e} \\
& C_{A_{\beta}}=\mathrm{G}(\mathbf{r}) \quad \text { a } \mathrm{t}=0
\end{aligned}
$$

Los términos diferentes de las desviaciones $\left(\tilde{C}_{A_{\alpha}}\right)$ o sus derivadas o combinaciones de estas son: $\frac{\partial\left\langle C_{A_{\beta}}\right\rangle^{\beta}}{\partial t}$ y $\nabla\left\langle C_{A_{\beta}}\right\rangle^{\beta}$. Lo cual sugiere que la solución para las desviaciones debe de ser una combinación de la forma:

$$
\tilde{C}_{A_{\beta}}=\mathbf{b} \cdot \nabla\left\langle C_{A_{\beta}}\right\rangle^{\beta}+S\left(\frac{\partial\left\langle C_{A_{\beta}}\right\rangle^{\beta}}{\partial t}\right)
$$

Sustituyendo (B-56) en (B-52) se tiene: 


$$
\begin{array}{r}
\mathbf{v}_{\beta} \cdot \nabla\left(\mathbf{b} \cdot \nabla\left\langle C_{A_{\beta}}\right\rangle^{\beta}\right)+\mathbf{v}_{\beta} \cdot \nabla\left[s\left(\frac{\partial\left\langle C_{A_{\beta}}\right\rangle^{\beta}}{\partial t}\right)\right]+\tilde{\mathbf{v}}_{\beta} \cdot \nabla\left\langle C_{A_{\beta}}\right\rangle^{\beta}=\mathrm{D}_{\beta} \nabla^{2} \mathbf{b} \cdot \nabla\left\langle C_{A_{\beta}}\right\rangle^{\beta} \\
+\mathrm{D}_{\beta} \nabla^{2} s\left(\frac{\partial\left\langle C_{A_{\beta}}\right\rangle^{\beta}}{\partial t}\right)+\frac{a_{v} k_{e q}}{\varepsilon_{\beta}} \frac{\partial\left\langle C_{A_{\beta}}\right\rangle^{\beta}}{\partial t}
\end{array}
$$

Obteniéndose los siguientes problemas para b y s:

$$
\begin{aligned}
& \mathbf{v}_{\beta} \cdot \nabla \mathbf{b}+\tilde{\mathbf{v}}_{\beta}=\mathrm{D}_{\beta} \nabla^{2} \mathbf{b} \\
& \mathbf{v}_{\beta} \cdot \nabla s=\mathrm{D}_{\beta} \nabla^{2} s+\frac{a_{v} k_{e q}}{\varepsilon_{\beta}}
\end{aligned}
$$

Para las condiciones frontera se sustituye (B-56) en (B-53)

$$
-\mathbf{n}_{\beta \sigma} \cdot \mathrm{D}_{\beta} \nabla\left(\mathbf{b} \cdot \nabla\left\langle C_{A_{\beta}}\right\rangle^{\beta}\right)-\mathbf{n}_{\beta \sigma} \cdot \mathrm{D}_{\beta} \nabla s\left(\frac{\partial\left\langle C_{A_{\beta}}\right\rangle^{\beta}}{\partial t}\right)=\mathbf{n}_{\beta \sigma} \cdot \mathrm{D}_{\beta} \nabla\left\langle C_{A_{\beta}}\right\rangle^{\beta}+k_{e q} \frac{\partial\left\langle C_{A_{\beta}}\right\rangle^{\beta}}{\partial t}
$$

Por lo que las condiciones de frontera para b y s son:

$$
\begin{array}{ll}
-\mathbf{n}_{\beta \sigma} \cdot \nabla \mathbf{b}=\mathbf{n}_{\beta \sigma} \quad \text { para los términos que involucran } & \nabla\left\langle C_{A_{\beta}}\right\rangle^{\beta} \\
-\mathbf{n}_{\beta \sigma} \cdot \nabla s=\frac{k_{e q .}}{D_{\beta}} \text { para los términos que involucran } & \frac{\partial\left\langle C_{A_{\beta}}\right\rangle^{\beta}}{\partial t}
\end{array}
$$

Las ecuaciones para $\mathbf{b}$ y $s$ incluyendo condiciones de frontera son:

Problema para $\mathbf{b}$

$$
\begin{array}{ll}
\mathbf{v}_{\beta} \cdot \nabla \mathbf{b}+\tilde{\mathbf{v}}_{\beta}=\mathrm{D}_{\beta} \nabla^{2} \mathbf{b} & \\
-\mathbf{n}_{\beta \sigma} \cdot \nabla \mathbf{b}=\mathbf{n}_{\beta \sigma} & \\
\mathbf{b}\left(\mathbf{r}_{i}+\mathbf{l}_{i}\right)=\mathbf{b}(\mathbf{r}) & \text { Periodicidad }
\end{array}
$$

Problema para $S$ 


$$
\begin{aligned}
& \mathbf{v}_{\beta} \cdot \nabla s=\mathrm{D}_{\beta} \nabla^{2} s+\frac{a_{v} K_{e q}}{\varepsilon_{\beta}} \\
& -\mathbf{n}_{\beta \sigma} \cdot \nabla s=\frac{K_{e q}}{\mathrm{D}_{\beta}} \\
& s\left(\mathbf{r}_{i}+\mathbf{l}_{i}\right)=s(\mathbf{r}) \quad \text { Periodicidad }
\end{aligned}
$$

Regresando a (B-13) y aplicando la solución para las desviaciones (B-56)

$$
\begin{aligned}
\varepsilon_{\beta}\left(1+\frac{a_{v} k_{e q}}{\varepsilon_{\beta}}\right) \frac{\partial\left\langle C_{A_{\beta}}\right\rangle^{\beta}}{\partial t}+\nabla \cdot\left(\varepsilon_{\beta}\left\langle\mathbf{v}_{\beta}\right\rangle^{\beta}\left\langle C_{A_{\beta}}\right\rangle^{\beta}\right) \\
+\nabla \cdot\left\langle\mathbf{v}_{\beta}\left(\mathbf{b} \cdot \nabla\left\langle C_{A_{\beta}}\right\rangle^{\beta}+s \frac{\partial\left\langle C_{A_{\beta}}\right\rangle^{\beta}}{\partial t}\right)\right\rangle \\
=\nabla \cdot\left\{\mathrm{D}_{\beta}\left[\varepsilon_{\beta} \nabla\left\langle C_{A_{\beta}}\right\rangle^{\beta}+\frac{1}{\mathrm{~V}} \int_{A_{\beta \sigma}} \mathbf{n}_{\beta \sigma}\left(\mathbf{b} \cdot \nabla\left\langle C_{A_{\beta}}\right\rangle^{\beta}+s \frac{\partial\left\langle C_{A_{\beta}}\right\rangle^{\beta}}{\partial t}\right) d A\right]\right\}
\end{aligned}
$$

Definiendo la difusividad efectiva mediante:

$$
\mathbf{D}_{\text {eff. }}=\mathrm{D}_{\beta}\left(\mathbf{I}+\frac{1}{\mathrm{~V}_{\beta}} \int_{A_{\beta \sigma}} \mathbf{n}_{\beta \sigma} \mathbf{b} d A\right)
$$

Sustituyendo (B-71) en (B-70) se tiene:

$$
\begin{gathered}
\varepsilon_{\beta}\left(1+\frac{a_{v} k_{e q}}{\varepsilon_{\beta}}\right) \frac{\partial\left\langle C_{A_{\beta}}\right\rangle^{\beta}}{\partial t}+\nabla \cdot\left(\varepsilon_{\beta}\left\langle\mathbf{v}_{\beta}\right\rangle^{\beta}\left\langle C_{A_{\beta}}\right\rangle^{\beta}\right)+\nabla \cdot\left\langle\mathbf{v}_{\beta} s \frac{\partial\left\langle C_{A_{\beta}}\right\rangle^{\beta}}{\partial t}\right\rangle \\
=\nabla \cdot\left(\varepsilon_{\beta} \mathbf{D}_{\text {eff. }} \cdot \nabla\left\langle C_{A_{\beta}}\right\rangle^{\beta}\right)+\nabla \cdot\left(\varepsilon_{\beta} \frac{1}{V_{\beta}} \int_{A_{\beta \sigma}} \mathbf{n}_{\beta \sigma}\left(\mathrm{D}_{\beta} s\right) d A \frac{\partial\left\langle C_{A_{\beta}}\right\rangle^{\beta}}{\partial t}\right) \\
-\nabla \cdot\left\langle\mathbf{v}_{\beta} \mathbf{b} \cdot \nabla\left\langle C_{A_{\beta}}\right\rangle^{\beta}\right\rangle
\end{gathered}
$$

Reordenando (B-72) se tiene: 


$$
\begin{aligned}
\varepsilon_{\beta}\left(1+\frac{a_{v} k_{e q}}{\varepsilon_{\beta}}\right) \frac{\partial\left\langle C_{A_{\beta}}\right\rangle^{\beta}}{\partial t} & +\nabla \cdot\left(\varepsilon_{\beta}\left\langle\mathbf{v}_{\beta}\right\rangle^{\beta}\left\langle C_{A_{\beta}}\right\rangle^{\beta}\right) \\
& =\nabla \cdot\left(\varepsilon_{\beta} \mathbf{D}^{*} \cdot \nabla\left\langle C_{A_{\beta}}\right\rangle^{\beta}\right)+\nabla \cdot\left(\varepsilon_{\beta} \mathbf{u}^{*} \frac{\partial\left\langle C_{A_{\beta}}\right\rangle^{\beta}}{\partial t}\right)
\end{aligned}
$$

Donde:

$$
\begin{gathered}
\mathbf{u}=\frac{1}{V_{\beta}} \int_{A_{\beta \sigma}} \mathbf{n}_{\beta \sigma}\left(\mathrm{D}_{\beta} s\right) d A \\
\mathbf{D}=-\left\langle\mathbf{v}_{\beta} \mathbf{b}\right\rangle^{\beta} \\
\mathbf{D}^{*}=\mathbf{D}_{\text {eff. }}+\mathbf{D} \\
\mathbf{u}^{\prime}=-\left\langle\mathbf{v}_{\beta} s\right\rangle^{\beta} \\
\mathbf{u}^{*}=\mathbf{u}+\mathbf{u}^{\prime}
\end{gathered}
$$

Finalmente se analizará el último término de la ecuación (B-73) para definir si se puede despreciar. De las ecuación (B-68), (B-74) y (B-77) se estiman el orden de magnitud de $s$, u y u' respectivamente:

$$
\begin{aligned}
& \mathbf{s}=\mathbf{O}\left(\frac{K_{e q} l_{\beta}}{\mathrm{D}_{\beta}}\right) \\
& \mathbf{u}=\mathbf{O}\left(K_{e q}\right) \\
& \mathbf{u}^{\prime}=\mathbf{O}\left(\frac{\mathbf{v}_{\beta} K_{e q} l_{\beta}}{\mathrm{D}_{\beta}}\right)
\end{aligned}
$$

Por lo que el orden de magnitud de $\mathbf{u}^{*}$ es:

$$
\mathbf{u}^{*}=\mathbf{O}\left(K_{e q}\left[1 \pm \frac{\mathbf{v}_{\beta} l_{\beta}}{\mathrm{D}_{\beta}}\right]\right)
$$

Ya que $\mathbf{u}^{*} \mathrm{y} \frac{\partial\left\langle C_{A}\right\rangle^{\beta}}{\partial t}$ son cantidades promedio, la longitud característica es $L$, esto da los estimados:

$$
\nabla \cdot\left(\mathbf{u}^{*} \frac{\partial\left\langle C_{A}\right\rangle^{\beta}}{\partial t}\right)=\mathbf{O}\left(\frac{K_{e q}}{L}\left[1 \pm \frac{\mathbf{v}_{\beta} l_{\beta}}{\mathrm{D}_{\beta}}\right] \frac{\partial\left\langle C_{A}\right\rangle^{\beta}}{\partial t}\right)
$$


De esto se puede concluir que el término de transporte convectivo es despreciable en comparación con el término adsortivo.

$$
\nabla \cdot\left(\varepsilon_{\beta} \mathbf{u}^{*} \frac{\partial\left\langle C_{A}\right\rangle^{\beta}}{\partial t}\right)<<a_{v} K_{e q} \frac{\partial\left\langle C_{A}\right\rangle^{\beta}}{\partial t}
$$

La ecuación final queda:

$$
\varepsilon_{\beta}\left(1+\frac{a_{v} K_{e q}}{\varepsilon_{\beta}}\right) \frac{\partial\left\langle C_{A}\right\rangle^{\beta}}{\partial t}+\nabla \cdot\left(\varepsilon_{\beta}\left\langle\mathbf{v}_{\beta}\right\rangle^{\beta}\left\langle C_{A}\right\rangle^{\beta}\right)=\nabla \cdot\left(\varepsilon_{\beta} \mathbf{D}^{*} \cdot \nabla\left\langle C_{A}\right\rangle^{\beta}\right)
$$

Esta última ecuación es la utilizada en el desarrollo de la condición de salto (A-42), con la ventaja que ahora se tienen las restricciones asociadas y una expresión para el tensor asociado con la difusividad. 


\section{Apéndice C}

\section{Desarrollo del modelo en dos dimensiones}

Para el estudio de sistemas fluido-medio poroso en dos dimensiones se cuenta con: trabajos que muestran resultados numéricos involucrando difusión doble para un sistema con un medio poroso en una de las paredes horizontales o bien para sistemas formados por una cavidad con fase fluida, debido a la flexibilidad del modelo tridimensional y al hecho de no contar con resultados que consideren el sistema especificado, en el presente trabajo se desarrolló un modelo en dos dimensiones que considera el proceso de difusión doble para una cavidad rectangular con un sistema fluido-medio poroso, los resultados de este modelo permiten realizar una comparación parcial con el modelo tridimensional.

A continuación se muestra el planteamiento de las ecuaciones del modelo en dos dimensiones así como la implantación del método numérico. El desarrollo se presenta para el modelo que considera la corrección de Brinkman a la ecuación de Darcy en la ecuación de cantidad de movimiento para la región porosa, sin embargo en la sección de condiciones de frontera se presentan las condiciones utilizadas en el modelo bidimensional para sus similares en tres dimensiones presentados en el Capítulo 2.

\section{C.1 Sistema}

El sistema bajo consideración consta de una cavidad rectangular con dimensiones $x_{0}, y_{0}$ como se muestra en la Figura C-1, los ejes $x$ y $y$ están orientados de tal forma que exista una inclinación de $\varphi$ y $\left[\left(\frac{\pi}{2}\right)-\varphi\right]$ grados respectivamente. La parte inferior de la cavidad se encuentra llena de un medio poroso, se asume fluido homogéneo. El medio poroso es saturado por el mismo fluido que se encuentra ubicado en la parte superior de la cavidad. El flujo bidimensional se asume laminar e incompresible, el fluido binario es Newtoniano, las fronteras de la cavidad son estacionarias, rígidas e impermeables, por lo que la masa total en la cavidad permanece constante y no se consideran fuentes internas de generación de calor en la cavidad. 


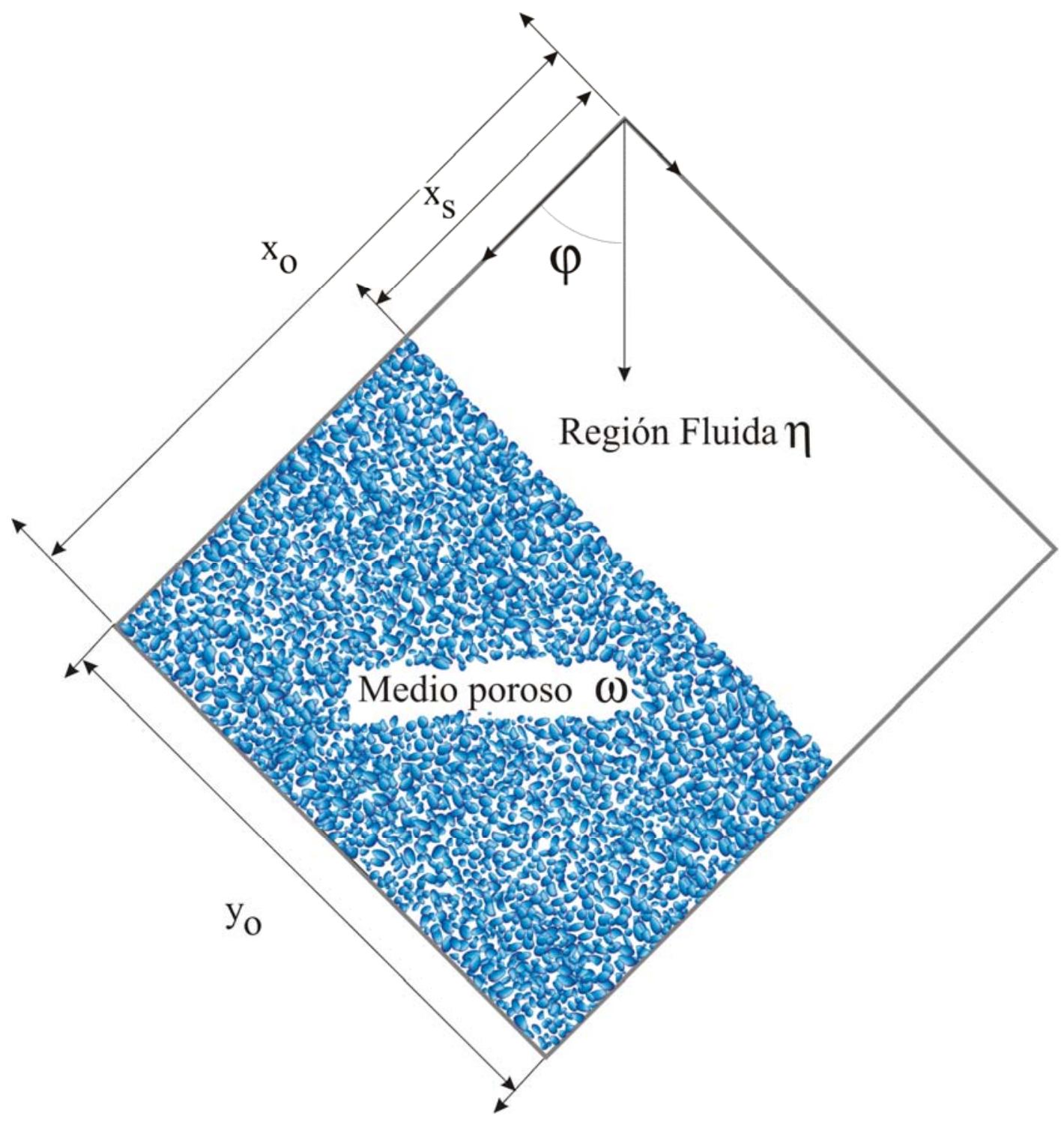

Figura C-1.- Sistema en dos dimensiones con inclinación $\varphi$. 


\section{C.2 Formulación matemática}

En e sistema considerado en este estudio, las paredes están sujetas a una diferencia de temperatura $\Delta T$ y concentración $\Delta C$ en la misma dirección. Para los efectos de variación de concentración y temperatura debido a variaciones de densidad se considera la aproximación de Boussinesq (ver sección 2.5), la cual es válida para pequeñas variaciones de la densidad como consecuencia de gradientes de temperatura y/o concentración a presión constante.

Las ecuaciones que se toman en cuenta para la continuidad, conservación de energía, masa y cantidad de movimiento son las versiones bidimensionales del modelo presentado en el Capítulo 2, realizando esta simplificación para cada una de las regiones se tiene las siguientes ecuaciones:

\section{REGIÓN FLUIDA $(\eta)$}

Continuidad:

$$
\frac{\partial\left\langle u_{\gamma}^{\prime}\right\rangle_{\eta}}{\partial x^{\prime}}+\frac{\partial\left\langle v_{\gamma}^{\prime}\right\rangle_{\eta}}{\partial y^{\prime}}=0
$$

Ecuación de energía:

$$
\left\langle u_{\gamma}^{\prime}\right\rangle_{\eta} \frac{\partial\left\langle T^{\prime}\right\rangle_{\eta}}{\partial x^{\prime}}+\left\langle v_{\gamma}^{\prime}\right\rangle_{\eta} \frac{\partial\left\langle T^{\prime}\right\rangle_{\eta}}{\partial y^{\prime}}=k_{\eta} \nabla^{\prime 2}\left\langle T^{\prime}\right\rangle_{\eta}
$$

Ecuación de transferencia de masa:

$$
\left\langle u_{\gamma}^{\prime}\right\rangle_{\eta} \frac{\partial\left\langle C_{A \gamma}^{\prime}\right\rangle_{\eta}}{\partial x^{\prime}}+\left\langle v_{\gamma}^{\prime}\right\rangle_{\eta} \frac{\partial\left\langle C_{A \gamma}^{\prime}\right\rangle_{\eta}}{\partial y^{\prime}}=\mathrm{D}_{\beta} \nabla^{\prime 2}\left\langle C_{A \gamma}^{\prime}\right\rangle_{\eta}
$$

Ecuaciones de movimiento:

Componente $x$

$$
\begin{aligned}
\left\langle u_{\gamma}^{\prime}\right\rangle_{\eta} \frac{\partial\left\langle u_{\gamma}^{\prime}\right\rangle_{\eta}}{\partial x^{\prime}}+\left\langle v_{\gamma}^{\prime}\right\rangle_{\eta} \frac{\partial\left\langle u_{\gamma}^{\prime}\right\rangle_{\eta}}{\partial y^{\prime}}= & -\frac{1}{\rho} \frac{\partial\left\langle p_{\gamma}^{\prime}\right\rangle_{\eta}}{\partial x^{\prime}}+\frac{\mu}{\rho} \nabla^{\prime 2}\left\langle u_{\gamma}^{\prime}\right\rangle_{\eta} \\
& -g \cos (\varphi)\left[\beta_{\theta}\left(\left\langle T^{\prime}\right\rangle-\left\langle T_{2}^{\prime}\right\rangle\right)+\beta_{\phi}\left(\left\langle C_{A}^{\prime}\right\rangle_{\eta}-\left\langle C_{A 2}^{\prime}\right\rangle_{\eta}\right)\right]
\end{aligned}
$$


Componente $y$

$$
\begin{aligned}
\left\langle u_{\gamma}^{\prime}\right\rangle_{\eta} \frac{\partial\left\langle v_{\gamma}^{\prime}\right\rangle_{\eta}}{\partial x^{\prime}}+\left\langle v_{\gamma}^{\prime}\right\rangle_{\eta} \frac{\partial\left\langle v_{\gamma}^{\prime}\right\rangle_{\eta}}{\partial y^{\prime}}= & -\frac{1}{\rho} \frac{\partial\left\langle p_{\gamma}^{\prime}\right\rangle_{\eta}}{\partial x^{\prime}}+\frac{\mu}{\rho} \nabla^{\prime 2}\left\langle v_{\gamma}^{\prime}\right\rangle_{\eta} \\
& -g \operatorname{sen}(\varphi)\left[\beta_{\theta}\left(\left\langle T^{\prime}\right\rangle-\left\langle T_{2}^{\prime}\right\rangle\right)+\beta_{\phi}\left(\left\langle C_{A}^{\prime}\right\rangle_{\eta}-\left\langle C_{A 2}^{\prime}\right\rangle_{\eta}\right)\right]
\end{aligned}
$$

REGIÓN POROSA $(\omega)$

Continuidad:

$$
\frac{\partial\left\langle u_{\gamma}^{\prime}\right\rangle_{\omega}}{\partial x^{\prime}}+\frac{\partial\left\langle v_{\gamma}^{\prime}\right\rangle_{\omega}}{\partial y^{\prime}}=0
$$

Ecuación de transferencia de energía:

$$
\left\langle u_{\gamma}^{\prime}\right\rangle_{\omega} \frac{\partial\left\langle T^{\prime}\right\rangle_{\omega}}{\partial x^{\prime}}+\left\langle v_{\gamma}^{\prime}\right\rangle_{\omega} \frac{\partial\left\langle T^{\prime}\right\rangle_{\omega}}{\partial y^{\prime}}=k_{\omega} \nabla^{\prime 2}\left\langle T^{\prime}\right\rangle_{\omega}
$$

Ecuación de transferencia de masa:

$$
\left\langle u_{\gamma}^{\prime}\right\rangle_{\omega} \frac{\partial\left\langle C_{A \gamma}^{\prime}\right\rangle_{\omega}}{\partial x^{\prime}}+\left\langle v_{\gamma}^{\prime}\right\rangle_{\omega} \frac{\partial\left\langle C_{A \gamma}^{\prime}\right\rangle_{\omega}}{\partial y^{\prime}}=D_{\beta} \nabla^{\prime 2}\left\langle C_{A \gamma}^{\prime}\right\rangle_{\omega}
$$

Ecuaciones de movimiento:

Componente $x$

$$
\begin{aligned}
\left\langle u_{\gamma}^{\prime}\right\rangle_{\omega} \frac{\partial\left\langle u_{\gamma}^{\prime}\right\rangle_{\omega}}{\partial x^{\prime}}+\left\langle v_{\gamma}^{\prime}\right\rangle_{\omega} \frac{\partial\left\langle u_{\gamma}^{\prime}\right\rangle_{\omega}}{\partial y^{\prime}}= & -\frac{1}{\rho} \frac{\partial\left\langle p_{\gamma}^{\prime}\right\rangle_{\omega}}{\partial x^{\prime}}+\frac{\mu}{\rho} \nabla^{\prime 2}\left\langle u_{\gamma}^{\prime}\right\rangle_{\omega} \\
& -g \cos (\varphi)\left[\beta_{\theta}\left(\left\langle T^{\prime}\right\rangle-\left\langle T_{2}^{\prime}\right\rangle\right)+\beta_{\phi}\left(\left\langle C_{A}^{\prime}\right\rangle_{\omega}-\left\langle C_{A 2}^{\prime}\right\rangle_{\omega}\right)\right]
\end{aligned}
$$

Componente $y$

$$
\begin{aligned}
\left\langle u_{\gamma}^{\prime}\right\rangle_{\omega} \frac{\partial\left\langle v_{\gamma}^{\prime}\right\rangle_{\omega}}{\partial x^{\prime}}+\left\langle v_{\gamma}^{\prime}\right\rangle_{\omega} \frac{\partial\left\langle v_{\gamma}^{\prime}\right\rangle_{\omega}}{\partial y^{\prime}} & =-\frac{1}{\rho} \frac{\partial\left\langle p_{\gamma}^{\prime}\right\rangle_{\omega}}{\partial x^{\prime}}+\frac{\mu}{\rho} \nabla^{\prime 2}\left\langle v_{\gamma}^{\prime}\right\rangle_{\omega} \\
& -g \operatorname{sen}(\varphi)\left[\beta_{\theta}\left(\left\langle T^{\prime}\right\rangle-\left\langle T_{2}^{\prime}\right\rangle\right)+\beta_{\phi}\left(\left\langle C_{A}^{\prime}\right\rangle_{\omega}-\left\langle C_{A 2}^{\prime}\right\rangle_{\omega}\right)\right]
\end{aligned}
$$

Donde $\left\langle T_{\gamma}^{\prime}\right\rangle_{\eta}$ representa la temperatura local de equilibrio del fluido y el medio poroso, (Whitaker, 1992b), mientras que $\left\langle C_{A}^{\prime}\right\rangle$ es la concentración del constituyente de interés, o explícitamente el número de kilogramos de constituyente por unidad de volumen de medio poroso (sólido y fluido) 


\section{C.3 Formulación vorticidad-función de corriente.}

Con la formulación anterior se tendrán las variables $\left\langle u_{\gamma}^{\prime}\right\rangle_{\lambda},\left\langle v_{\gamma}^{\prime}\right\rangle_{\lambda},\left\langle p_{\gamma}^{\prime}\right\rangle_{\lambda},\left\langle T^{\prime}\right\rangle_{\lambda} \mathrm{y}$ $\left\langle C_{A}^{\prime}\right\rangle_{\lambda}$ (variables primitivas), mientras que si no se hubiera utilizado la aproximación de Boussinesq se tendría que incluir la dependencia densidad, además, en un enfoque más riguroso, las propiedades termodinámicas involucradas en las ecuaciones de transporte como la viscosidad, conductividad térmica y difusividad también están en función de la temperatura, por lo que la estructura de las ecuaciones se complicaría mas y como consecuencia su solución numérica.

Para evitar resolver de manera directa el problema de cantidad de movimiento en términos de la presión, se plantean las ecuaciones de movimiento en términos de la vorticidad $\left(\zeta^{\prime}\right)$ y la función de corriente $\left(\Psi^{\prime}\right)$, las relaciones entre estas variables con respecto al vector velocidad en un sistema bidimensional quedan especificadas mediante las siguientes expresiones:

$$
\begin{aligned}
& \zeta_{\lambda}^{\prime}=\frac{\partial\left\langle v_{\gamma}^{\prime}\right\rangle_{\lambda}}{\partial x^{\prime}}-\frac{\partial\left\langle u_{\gamma}^{\prime}\right\rangle_{\lambda}}{\partial y^{\prime}} \quad \text { para } \quad \lambda=\omega, \eta \\
& \left.\begin{array}{l}
\left\langle u_{\gamma}^{\prime}\right\rangle_{\lambda}=\frac{\partial \Psi_{\lambda}^{\prime}}{\partial y^{\prime}} \\
\left\langle v_{\gamma}^{\prime}\right\rangle_{\lambda}=-\frac{\partial \Psi_{\lambda}^{\prime}}{\partial x^{\prime}}
\end{array}\right\} \quad \text { para } \lambda=\omega, \eta
\end{aligned}
$$

Obteniendo una relación de vorticidad con respecto a la función de corriente mediante las ecuaciones (C-11) y (C-12)

$$
\begin{array}{cc}
\zeta_{\lambda}^{\prime}=\frac{\partial^{2}\left\langle\Psi_{\lambda}^{\prime}\right\rangle}{\partial x^{2}}-\frac{\partial^{2}\left\langle\Psi_{\lambda}^{\prime}\right\rangle}{\partial y^{2}} & \text { para } \lambda=\omega, \eta \\
\zeta_{\lambda}^{\prime}=-\nabla^{2} \Psi^{\prime} & \text { para } \lambda=\omega, \eta
\end{array}
$$

La combinación de las ecuaciones de transferencia de movimiento en ambas regiones, por diferenciación cruzada de las ecuaciones (C-4), (C-5), (C-9) y (C-10), la resta junto con la definición bidimensional de vorticidad da como resultado las ecuaciones en términos del vector vorticidad: 
REGIÓN FLUIDA $(\eta)$

$$
\begin{aligned}
\left\langle u_{\gamma}^{\prime}\right\rangle_{\eta} \frac{\partial\left\langle u_{\gamma}^{\prime}\right\rangle_{\eta}}{\partial x^{\prime}}+\left\langle v_{\gamma}^{\prime}\right\rangle_{\eta} \frac{\partial\left\langle u_{\gamma}^{\prime}\right\rangle_{\eta}}{\partial y^{\prime}}= & -\frac{1}{\rho} \frac{\partial\left\langle p_{\gamma}^{\prime}\right\rangle_{\eta}}{\partial x^{\prime}}+\frac{\mu}{\rho} \nabla^{\prime 2}\left\langle u_{\gamma}^{\prime}\right\rangle_{\eta} \\
& -g \cos (\varphi)\left[\beta_{\theta}\left(\left\langle T^{\prime}\right\rangle-\left\langle T_{2}^{\prime}\right\rangle\right)+\beta_{\phi}\left(\left\langle C_{A}^{\prime}\right\rangle_{\eta}-\left\langle C_{A 2}^{\prime}\right\rangle_{\eta}\right)\right]
\end{aligned}
$$

REGIÓN POROSA $(\omega)$

$$
\begin{aligned}
\left\langle u_{\gamma}^{\prime}\right\rangle_{\eta} \frac{\partial\left\langle v_{\gamma}^{\prime}\right\rangle_{\eta}}{\partial x^{\prime}}+\left\langle v_{\gamma}^{\prime}\right\rangle_{\eta} \frac{\partial\left\langle v_{\gamma}^{\prime}\right\rangle_{\eta}}{\partial y^{\prime}}= & -\frac{1}{\rho} \frac{\partial\left\langle p_{\gamma}^{\prime}\right\rangle_{\eta}}{\partial x^{\prime}}+\frac{\mu}{\rho} \nabla^{\prime 2}\left\langle v_{\gamma}^{\prime}\right\rangle_{\eta} \\
& -g \operatorname{sen}(\varphi)\left[\beta_{\theta}\left(\left\langle T^{\prime}\right\rangle-\left\langle T_{2}^{\prime}\right\rangle\right)+\beta_{\phi}\left(\left\langle C_{A}^{\prime}\right\rangle_{\eta}-\left\langle C_{A 2}^{\prime}\right\rangle_{\eta}\right)\right]
\end{aligned}
$$

\section{C.4 Ecuaciones adimensionales.}

El uso de variables adimensionales es deseable ya que esto reduce el número de parámetros independientes que son necesarios (o alternativamente incrementa la generalidad de la solución para un número dado de parámetros).

Para expresar la formulación en forma adimensional se utilizan las variables adimensionales previamente definidas en el Capítulo 2, junto con la adimensionalización de las longitudes:

$$
\begin{aligned}
& x=\frac{x^{\prime}}{L} \\
& y=\frac{y^{\prime}}{L}
\end{aligned}
$$

Las variables y números adimensionales utilizadas previamente, se resumen en las tablas C-1 y C-2.

A partir de aquí y para evitar sobrecargar la nomenclatura las variables promedio se mostrarán en forma simplificada evitando el símbolo “ \langle\rangle ”, de tal manera que las ecuaciones adimensionales en términos de la vorticidad-función de corriente para cada región son: 


\begin{tabular}{|c|c|l|}
\hline Variable & Expresión & Ecuación \\
\hline \hline$\nabla$ & $\nabla=L_{x} \nabla^{\prime}$ & $(2.4 \mathrm{a})$ \\
\hline$\nabla^{2}$ & $\nabla^{2}=L_{x}^{2} \nabla^{\prime 2}$ & $(2.4 \mathrm{~b})$ \\
\hline $\mathbf{v}_{\lambda}$ & $\mathbf{v}_{\lambda}=\frac{\left\langle\mathbf{v}_{\beta}^{\prime}\right\rangle_{\lambda} L_{x}}{\left(\alpha_{\beta}\right)_{\lambda}}$ & $(2.2)$ \\
\hline$\theta_{\lambda}$ & $\theta_{\lambda}=\frac{\left\langle T_{\beta}\right\rangle_{\lambda}-T_{\text {ref }}}{\Delta T}$ & $(2.3)$ \\
\hline$\phi_{\lambda}$ & $\phi_{\lambda}=\frac{\left\langle C_{A \beta}\right\rangle_{\lambda}-C_{A \beta_{\text {ref }}}}{\Delta C}$ & $(2.13)$ \\
\hline$p_{\lambda}$ & $p_{\lambda}=\frac{\left\langle p_{\beta}^{\prime}\right\rangle_{\lambda} L_{x}^{2}}{\left(\alpha_{\beta}\right)_{\lambda} \mu_{\beta}}$ & $(2.21)$ \\
\hline$\zeta_{\lambda}$ & $\zeta_{\lambda}=\nabla \times \mathbf{v}_{\lambda}$ & $(2.37)$ \\
\hline
\end{tabular}

Tabla C-1. Variables adimensionales

\begin{tabular}{|c|c|l|}
\hline No. Adimensional & Expresión & Ecuación \\
\hline \hline$D a$ & $D a=\frac{K}{L_{x}^{2}}$ & $(2.31)$ \\
\hline$R a_{\theta}$ & $R a_{\theta}=\frac{g \beta_{\theta} \Delta T L_{x}^{3}}{\left(\alpha_{\beta}\right)_{\eta} v_{\beta}}$ & $(2.23)$ \\
\hline$R a_{\phi}$ & $R a_{\phi}=\frac{g \beta_{\phi} \Delta C_{A} L_{x}^{3}}{\left(D_{\beta}\right)_{\eta} v_{\beta}}$ & $(2.23 a)$ \\
\hline$P r$ & $P r=\frac{v_{\beta}}{\left(\alpha_{\beta}\right)_{\eta}}=\frac{\rho_{\beta}}{\rho_{\beta}\left(\alpha_{\beta}\right)}$ & $(2.24)$ \\
\hline$L e$ & $L e_{\beta}=\frac{\left(\alpha_{\beta}\right)_{\eta}}{D_{e f f}}$ & $(2.17)$ \\
\hline
\end{tabular}

Tabla C-2. Números adimensionales 


\section{REGIÓN FLUIDA $(\eta)$}

Ecuación de energía:

$$
\frac{\partial\left(u_{\eta} \theta_{\eta}\right)}{\partial x}+\frac{\partial\left(v_{\eta} \theta_{\eta}\right)}{\partial y}=k_{\eta} \nabla^{2} \theta_{\eta}
$$

Ecuación de transferencia de masa:

$$
\frac{\partial\left(u_{\eta} \phi_{\eta}\right)}{\partial x}+\frac{\partial\left(v_{\eta} \phi_{\eta}\right)}{\partial y}=\frac{1}{L e_{\eta}} \nabla^{2} \phi_{\eta}
$$

Ecuación de movimiento:

$$
\begin{aligned}
\frac{1}{\operatorname{Pr}}\left[\frac{\partial\left(u_{\eta} \zeta_{\eta}\right)}{\partial x}+\frac{\partial\left(v_{\eta} \zeta_{\eta}\right)}{\partial y}\right]=\nabla^{2} u_{\eta} & -R_{\theta}\left(\operatorname{sen}(\varphi) \frac{\partial \theta_{\eta}}{\partial y}+\cos (\varphi) \frac{\partial \theta_{\eta}}{\partial x}\right) \\
& -\frac{R a_{\phi}}{L e_{\eta}}\left(\operatorname{sen}(\varphi) \frac{\partial \phi_{\eta}}{\partial y}+\cos (\varphi) \frac{\partial \phi_{\eta}}{\partial x}\right)
\end{aligned}
$$

\section{REGIÓN POROSA $(\omega)$}

Ecuación de energía:

$$
\frac{\partial\left(u_{\omega} \theta_{\omega}\right)}{\partial x}+\frac{\partial\left(v_{\omega} \theta_{\omega}\right)}{\partial y}=k_{\omega} \nabla^{2} \theta_{\omega}
$$

Ecuación de transferencia de masa:

$$
\frac{\partial\left(u_{\omega} \phi_{\omega}\right)}{\partial x}+\frac{\partial\left(v_{\omega} \phi_{\omega}\right)}{\partial y}=\frac{1}{L e_{\omega}} \nabla^{2} \phi_{\omega}
$$

Ecuación de movimiento:

Para la formulación que utiliza la corrección de Brinkman a la ecuación de Darcy (ver sección 2.5.2) se tiene:

$$
\begin{aligned}
\frac{1}{\operatorname{Pr}}\left[\frac{\partial\left(u_{\omega} \zeta_{\omega}\right)}{\partial x^{\prime}}+\frac{\partial\left(v_{\omega} \zeta_{\omega}\right)}{\partial y^{\prime}}\right]=\nabla^{2} u_{\omega} & -R a_{\theta}\left(\operatorname{sen}(\varphi) \frac{\partial \theta_{\omega}}{\partial y}+\cos (\varphi) \frac{\partial \theta_{\omega}}{\partial x}\right) \\
& \left.-\frac{R a_{\phi}\left(\operatorname{sen}(\varphi) \frac{\partial \phi_{\omega}}{\partial y}+\cos (\varphi) \frac{\partial \phi_{\omega}}{\partial x}\right)}{L e_{\omega}}\right)
\end{aligned}
$$




\section{C.5 Condiciones en las paredes del sistema}

Para las condiciones de frontera en las paredes para la temperatura y concentración, el modelo tiene la opción de considerar los mismos tipos de condiciones especificados para el modelo en tres dimensiones (Newman, Dirichlet y Cauchy, ver sección 2.3.3), de tal forma que las condiciones utlizadas para la comparación con el modelo tridimensional son:

$$
\begin{aligned}
& \theta=1, \quad \phi=1 \quad \text { a } \quad Y=0 \quad \text { y } \quad X_{\mathrm{a}} \leq X \leq X_{\mathrm{b}} \\
& \frac{\partial \theta}{\partial Y}=0, \quad \frac{\partial \phi}{\partial Y}=0 \quad \text { a } \quad Y=0 \quad y \quad\left\{\begin{array}{c}
0 \leq X<X_{\mathrm{a}} \\
X_{\mathrm{b}}<X \leq X_{0}
\end{array}\right. \\
& \theta=0, \quad \phi=0 \quad \text { a } \quad Y=1 \quad \text { y } \quad X_{\mathrm{c}} \leq X \leq X_{\mathrm{d}} \\
& \frac{\partial \theta}{\partial Y}=0, \quad \frac{\partial \phi}{\partial Y}=0 \quad \text { a } \quad Y=1 \quad \text { y } \quad\left\{\begin{array}{c}
0 \leq X<X_{\mathrm{c}} \\
X_{\mathrm{d}}<X \leq X_{0}
\end{array}\right.
\end{aligned}
$$

\section{CONDICIONES DE FRONTERA PARA LA FUNCIÓN DE CORRIENTE}

Para el caso en que las paredes son estacionarias, no existe deslizamiento ni penetración, las condiciones de frontera en términos de variables primitivas son:

$$
u_{b}=v_{b}=0
$$

Donde el subíndice $b$ denota el valor en la pared de la cavidad, sustituyendo en la ecuación (C-12) se obtienen las condiciones de frontera en términos de la función de corriente:

$$
\begin{aligned}
& \Psi_{b}=\text { constante } \\
& \left(\frac{\partial \Psi}{\partial n}\right)_{b}=0
\end{aligned}
$$




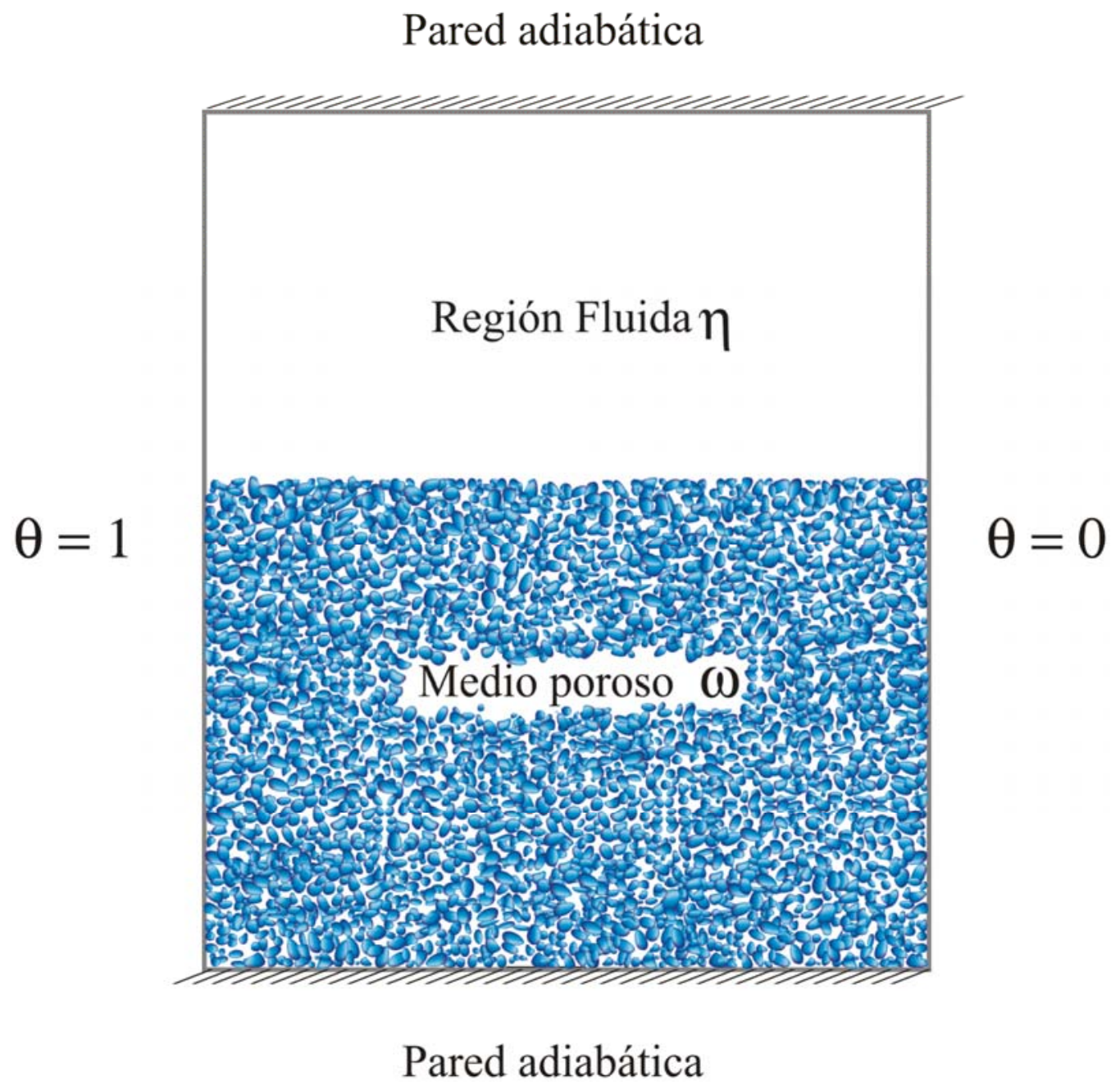

Figura C.2 Notación para condiciones de frontera en la transferencia de energía. 
Donde $n$ indica la dirección normal a la pared, el valor de la constante en la ecuación (C-28) es un valor arbitrario y puede tomar el valor de cero.

\section{CONDICIONES DE FRONTERA PARA LA VORTICIDAD}

Las condiciones de frontera para la vorticidad se obtienen de la ecuación (C-13a), las ecuaciones en las paredes son

$$
\zeta_{b}=-\left(\frac{\partial^{2} \Psi}{\partial f^{2}}+\frac{\partial^{2} \Psi}{\partial n^{2}}\right)_{b}
$$

Donde los subíndices $f$ y $n$ indican las coordenadas tangencial y normal a la pared respectivamente. De la ecuación (C-28) se observa que cualquier derivada de $\Psi$ en la dirección tangencial es cero, con lo anterior la ecuación (C-30) queda:

$$
\left(\frac{\partial \Psi}{\partial f}\right)_{b}=\left(\frac{\partial^{2} \Psi}{\partial f^{2}}\right)_{b}=0
$$

Cuando se sustituye la ecuación (C-31) en (C-30) se obtiene la condición de frontera para la vorticidad en términos de la función de corriente

$$
\zeta_{b}=-\left(\frac{\partial^{2} \Psi}{\partial n^{2}}\right)_{b}
$$

\section{CONDICIONES EN LA INTERREGIÓN.}

Las condiciones interregionales en su forma adimensional para la ecuación de energía quedan determinadas por la continuidad de la temperatura y del flux:

$$
\begin{array}{ll}
\left\langle T^{\prime}\right\rangle_{\omega}=\left\langle T_{\gamma}^{\prime}\right\rangle_{\eta} & \text { en } x^{\prime}=x_{s}^{\prime} \\
-\mathbf{n}_{\omega \eta} \cdot \mathbf{K}_{\omega} \cdot \nabla^{\prime}\left\langle T^{\prime}\right\rangle_{\omega}=-\mathbf{n}_{\omega \eta} \cdot k_{\gamma} \nabla^{\prime}\left\langle T_{\gamma}^{\prime}\right\rangle_{\eta} & \text { en } x^{\prime}=x_{s}^{\prime}
\end{array}
$$

Para la ecuación de cantidad de movimiento las condiciones interregionales están especificadas por la continuidad de la velocidad en la dirección normal para el modelo que utiliza la corrección de Brinkman:

$$
\langle u\rangle_{\omega}=\langle u\rangle_{\eta}
$$


Y para el modelo que utiliza la ecuación de Darcy con la corrección de Beavers y Joseph

$$
\frac{\partial}{\partial y}\langle v\rangle_{\eta}=\alpha\left[\langle v\rangle_{\eta}-\langle v\rangle_{\omega}\right]
$$

Donde

$$
\alpha=\frac{\delta}{\sqrt{K_{\beta \omega}}}
$$

\section{C.6 Método numérico.}

El método numérico que se adoptó para el caso bidimensional tiene mucha semejanza con el desarrollado para el modelo en tres dimensiones, pero también algunas limitaciones, una de ella es el espaciamiento entre nodos, en el caso del modelo bidimensional el espaciamiento es constante, a diferencia del utilizado en el modelo tridimensional, en el cual se puede modificar el espaciamiento, esta diferencia no causa gran problema, ya que los arreglos bidimensionales utilizados no consumen memoria de cómputo considerable.

Se cuenta con una red rectangular y uniforme en cada dirección, la red está compuesta por $m$ nodos en la dirección $X$ y $n$ en la dirección $Y$, el espaciamiento constante en cada dirección queda determinada por:

$$
\begin{aligned}
\Delta X & =\frac{1}{m-1} \\
\Delta Y & =\frac{A}{n-1}
\end{aligned}
$$

Donde $A=\frac{Y_{0}}{X_{0}}$ es la relación de aspecto de la cavidad

\section{DISCRETIZACIÓN DE LA ECUACIÓN DE ENERGÍA}

La ecuación de energía considerando la dependencia artificial del tiempo al utilizar el método del falso transiente (ver sección 3.2), adquiere la siguiente forma para ambas regiones:

$$
\frac{1}{\left(\alpha_{\theta}\right)_{\lambda}} \frac{\partial \theta}{\partial t}=-T_{i}\left\{\frac{\partial}{\partial X}\left(u_{\lambda} \theta_{\lambda}\right)+\frac{\partial}{\partial Y}\left(v_{\lambda} \theta_{\lambda}\right)\right\}+T_{d}\left\{\frac{\partial^{2} \theta_{\lambda}}{\partial X^{2}}\left(u_{\lambda}\right)+\frac{\partial^{2} \theta_{\lambda}}{\partial Y^{2}}\right\}
$$

Donde $\lambda=\eta, \omega$ y los valores de las variables $T_{i}$ y $T_{d}$ son: 


\begin{tabular}{|c|c|c|}
\hline Temperatura & Región $\eta$ & Región $\omega$ \\
\hline \hline$T_{i}$ & 1 & $\frac{k_{\omega}}{k_{\eta}}$ \\
\hline$T_{d}$ & 1 & 1 \\
\hline
\end{tabular}

Tabla C-3 Parámetros utilizados en la ecuación de energía

La forma discretizada de la ecuación (C-39) es la siguiente:

$$
\begin{aligned}
\frac{1}{\left(\alpha_{\theta}\right)_{\lambda}} \frac{\theta^{*}-\theta}{\frac{\Delta t}{2}}= & -T_{i}\left\{\frac{u_{(i+1)_{\lambda}} \theta_{(i+1)_{\lambda}}^{*}-u_{(i-1)_{\lambda}} \theta_{(i-1)_{\lambda}}^{*}}{2 \Delta X}+\frac{v_{(j+1)_{\lambda}} \theta_{(j+1)_{\lambda}}-v_{(j-1)_{\lambda}} \theta_{(j-1)_{\lambda}}}{2 \Delta Y}\right\} \\
& +T_{d}\left\{\frac{\theta_{(i+1)_{\lambda}}^{*}-2 \theta_{(i)_{\lambda}}^{*}+\theta_{(i-1)_{\lambda}}^{*}}{\Delta X^{2}}+\frac{\theta_{(j+1)_{\lambda}}-2 \theta_{(j)_{\lambda}}+\theta_{(j-1)_{\lambda}}}{\Delta Y^{2}}\right\}
\end{aligned}
$$

De acuerdo con la discretización propuesta por Peaceman y Rachford (1955), los valores intermedios(*) son evaluados por una aproximación en diferencias finitas, el cual es implícita en la dirección $X$ y explícito en la dirección $Y$, esto es, las derivadas en la dirección $X$ son encontradas usando los nuevos valores (en el tiempo intermedio) y las derivadas en $Y$ usando los valores anteriores, la ecuación discretizada mediante diferencias finitas esta definida mediante el reagrupamiento: en términos de $\theta^{*}$

$$
\begin{aligned}
&\left\{-\frac{\left(\alpha_{\theta}\right)_{\lambda} T_{i} u_{(i-1)_{\lambda}}}{2 \Delta X}-\frac{\left(\alpha_{\theta}\right)_{\lambda} T_{d}}{\Delta X^{2}}\right\} \theta_{(i-1)_{\lambda}}^{*}+\left\{\frac{2}{\Delta t}+\frac{2\left(\alpha_{\theta}\right)_{\lambda} T_{d}}{\Delta X^{2}}\right\} \theta_{(i)_{\lambda}}^{*} \\
&+\left\{\frac{\left(\alpha_{\theta}\right)_{\lambda} T_{i} u_{(i+1)_{\lambda}}}{2 \Delta X}-\frac{\left(\alpha_{\theta}\right)_{\lambda} T_{d}}{\Delta X^{2}}\right\} \theta_{(i+1)_{\lambda}}^{*}\left\{\frac{\left(\alpha_{\theta}\right)_{\lambda} T_{i} u_{(j-1)_{\lambda}}}{2 \Delta Y}+\frac{\left(\alpha_{\theta}\right)_{\lambda} T_{d}}{\Delta Y^{2}}\right\} \theta_{(j-1)_{\lambda}} \\
&+\left\{\frac{2}{\Delta t}+\frac{2\left(\alpha_{\theta}\right)_{\lambda} T_{d}}{\Delta Y^{2}}\right\} \theta_{(j)_{\lambda}} \\
&+\left\{-\frac{\left(\alpha_{\theta}\right)_{\lambda} T_{i} u_{(j+1)_{\lambda}}}{2 \Delta Y}+\frac{\left(\alpha_{\theta}\right)_{\lambda} T_{d}}{\Delta Y^{2}}\right\} \theta_{(j+1)_{\lambda}}
\end{aligned}
$$

Para cualquier valor de " $j$ " y asumiendo que $\theta_{1}$ y $\theta_{m}$ son conocidos de la condición de frontera en la iteración anterior, la ecuación (C-42) es la $(j-1)^{\text {ava }}$ de un total de $(m-2)$ ecuaciones simultaneas para $(m-2)$ variables. 


$$
A_{i-1} \theta_{i-1}^{*}+B_{i-1} \theta^{*}+C_{i-1} \theta_{i+1}^{*}=D_{i-1} \quad \text { para } i=2, \ldots m-1
$$

Donde:

$$
\begin{array}{ll}
A_{i-1}=-C_{T 1} u_{i-1}-C_{T 2} & \text { para } i=2, \ldots m-1 \\
B_{i-1}=C_{T 3} & \text { para } i=2, \ldots m-1 \\
C_{i-1}=C_{T 1} u_{i+1}-C_{T 2} & \text { para } i=2, \ldots m-1 \\
D_{i-1}=\left(C_{T 4} v_{j-1}+C_{T 5}\right) \theta_{(j-1)_{\lambda}}+C_{T 6} \theta_{\lambda}+\left(-C_{T 4} v_{j+1}+C_{T 5}\right) \theta_{(j+1)_{\lambda}} & \text { para } i=2, \ldots m-1
\end{array}
$$

Donde las constantes que involucran incrementos en la dirección $x$ :

$$
\begin{aligned}
C_{T 1} & =\frac{\alpha_{\theta} T_{i}}{2 \Delta X} \\
C_{T 2} & =\frac{\alpha_{\theta} T_{d}}{\Delta X^{2}} \\
C_{T 3} & =\frac{2\left[\Delta X^{2}+\alpha_{\theta} \Delta t T_{d}\right]}{\Delta X^{2} \Delta t} \\
C_{T 4} & =\frac{\alpha_{\theta} T_{i}}{2 \Delta Y}
\end{aligned}
$$

Y las constantes que involucran incrementos en la dirección y:

$$
\begin{aligned}
C_{T 5} & =\frac{\alpha_{\theta} T_{d}}{\Delta Y^{2}} \\
C_{T 6} & =\frac{2\left[\Delta Y^{2}-\alpha_{\theta} \Delta t T_{d}\right]}{\Delta Y^{2} \Delta t} \\
C_{T 7} & =\frac{2\left[\Delta Y^{2}+\alpha_{\theta} \Delta t T_{d}\right]}{\Delta Y^{2} \Delta t} \\
C_{T 8} & =\frac{2\left[\Delta X^{2}-\alpha_{\theta} \Delta t T_{d}\right]}{\Delta X^{2} \Delta t}
\end{aligned}
$$

El sistema tridiagonal se resuelve mediante el algoritmo de Thomas comenzando un nodo después de la condición de frontera y terminando un nodo antes de $X=X_{s}$ para la parte porosa, para los valores de la interregión se toman los valores obtenidos de la iteración anterior (la actualización de los valores de la interregión se realiza al evaluar las condiciones de frontera), posteriormente se continua con la región fluida hasta un nodo antes de la condición de frontera. Terminado el barrido en la dirección $X$ en la región fluida y con el 
valor del campo para $\theta^{*}$, se continúa con el segundo paso en el método de Peaceman y Rachford (1955), el cual considera las derivadas implícitas en la dirección $Y$.

$$
\begin{aligned}
\frac{1}{\left(\alpha_{\theta}\right)_{\lambda}} \frac{\theta^{k+1}-\theta^{*}}{\frac{\Delta t}{2}=} & -T_{i}\left\{\frac{u_{(i+1)_{\lambda}} \theta_{(i+1)_{\lambda}}^{*}-u_{(i-1)_{\lambda}} \theta_{(i-1)_{\lambda}}^{*}}{2 \Delta X}+\frac{v_{(j+1)_{\lambda}} \theta_{(j+1)_{\lambda}}^{k+1}-v_{(j-1)_{\lambda}} \theta_{(j-1)_{\lambda}}^{k+1}}{2 \Delta Y}\right\} \\
& +T_{d}\left\{\frac{\theta_{(i+1)_{\lambda}}^{*}-2 \theta_{(i)_{\lambda}}^{*}+\theta_{(i-1)_{\lambda}}^{*}}{\Delta X^{2}}+\frac{\theta_{(j+1)_{\lambda}}^{k+1}-2 \theta_{(j)_{\lambda}}^{k+1}+\theta_{(j-1)_{\lambda}}^{k+1}}{\Delta Y^{2}}\right\}
\end{aligned}
$$

Reagrupando en términos de $\theta^{k+1}$ :

$$
\begin{aligned}
\left\{-\frac{\left(\alpha_{\theta}\right)_{\lambda} T_{i} v_{(i-1)_{\lambda}}}{2 \Delta Y}-\frac{\left(\alpha_{\theta}\right)_{\lambda} T_{d}}{\Delta Y^{2}}\right\} \theta_{(j-1)_{\lambda}}^{k+1}+\left\{\frac{2}{\Delta t}+\frac{2\left(\alpha_{\theta}\right)_{\lambda} T_{d}}{\Delta Y^{2}}\right\} \theta_{(i)_{\lambda}}^{k+1} & \\
+\left\{\frac{\left(\alpha_{\theta}\right)_{\lambda} T_{i} v_{(i+1)_{\lambda}}}{2 \Delta Y}-\frac{\left(\alpha_{\theta}\right)_{\lambda} T_{d}}{\Delta Y^{2}}\right\} \theta_{(j+1)_{\lambda}}^{k+1} & =\left\{\frac{\left(\alpha_{\theta}\right)_{\lambda} T_{i} u_{(i-1)_{\lambda}}}{2 \Delta X}+\frac{\left(\alpha_{\theta}\right)_{\lambda} T_{d}}{\Delta X^{2}}\right\} \theta_{(i-1)_{\lambda}}^{*} \\
& +\left\{\frac{2}{\Delta t}+\frac{2\left(\alpha_{\theta}\right)_{\lambda} T_{d}}{\Delta X^{2}}\right\} \theta_{(i)_{\lambda}}^{*} \\
& +\left\{-\frac{\left(\alpha_{\theta}\right)_{\lambda} T_{i} u_{(i+1)_{\lambda}}}{2 \Delta X}+\frac{\left(\alpha_{\theta}\right)_{\lambda} T_{d}}{\Delta X^{2}}\right\} \theta_{(i+1)_{\lambda}}^{*}
\end{aligned}
$$

Para cualquier valor de " $i$ " y asumiendo que $\theta_{1}$ y $\theta_{n}$ son conocidos de la condición de frontera , la ecuación (C-58) es la $(i-1)^{\text {ava }}$ de un total de $(n-2)$ ecuaciones simultaneas para $(n-2)$ variables.

$$
A_{j-1} \theta_{j-1}^{k+1}+B_{j-1} \theta^{k+1}+C_{j-1} \theta_{j+1}^{k+1}=D_{j-1} \quad \text { para } i=2, \ldots m-1
$$

Donde:

$$
\begin{array}{ll}
A_{j-1}=-C_{T 4} u_{j-1}-C_{T 5} & \text { para } i=2, \ldots m-1 \\
B_{j-1}=C_{T 7} & \text { para } i=2, \ldots m-1 \\
C_{j-1}=C_{T 4} v_{j+1}-C_{T 5} & \text { para } i=2, \ldots m-1 \\
D_{j-1}=\left(C_{T 1} u_{i-1}+C_{T 2}\right) \theta_{(i-1)_{\lambda}}^{*}+C_{T 8} \theta_{\lambda}^{*}+\left(-C_{T 1} u_{i+1}+C_{T 2}\right) \theta_{(i+1)_{\lambda}}^{*} & \text { para } i=2, \ldots m-1
\end{array}
$$

\section{DISCRETIZACIÓN DE LA ECUACIÓN DE VORTICIDAD}

La ecuación de vorticidad (C-19), (C-22) incluyendo el término artificial del tiempo, para cualquier región queda: 


$$
\begin{aligned}
& \frac{1}{\alpha_{\zeta}} \frac{\partial \zeta_{\lambda}}{\partial t}+\frac{1}{\operatorname{Pr}}\left[\frac{\partial\left(u_{\lambda} \zeta_{\lambda}\right)}{\partial X}+\frac{\partial\left(v_{\lambda} \zeta_{\lambda}\right)}{\partial Y}\right]=\nabla^{2} u_{\lambda}-\operatorname{Ra}_{\theta}\left(\operatorname{sen}(\varphi) \frac{\partial \theta_{\lambda}}{\partial Y}+\cos (\varphi) \frac{\partial \theta_{\lambda}}{\partial X}\right) \\
& -\frac{R a_{\phi}}{L e}\left(\operatorname{sen}(\varphi) \frac{\partial \phi_{\lambda}}{\partial y}+\cos (\varphi) \frac{\partial \phi_{\lambda}}{\partial x}\right)
\end{aligned}
$$

Las dos ecuaciones resultantes al aplicar la discretización propuesta por PeacemanRachford a la ecuación de vorticidad son:

Implícito en $X$

$$
\begin{aligned}
& \left(-C_{Z 1} u_{(i-1)_{\lambda}}-C_{Z 2}\right) \zeta_{(i-1)_{\lambda}}^{*}+C_{Z 3} \zeta_{(i)_{\lambda}}^{*}+\left(C_{Z 1} u_{(i+1)_{\lambda}}-C_{Z 2}\right) \zeta_{(i+1)_{\lambda}}^{*}=\left(C_{Z 4} v_{(j-1)_{\lambda}}+C_{Z 5}\right) \zeta_{(j-1)_{\lambda}} \\
& +C_{Z 6} \zeta_{(i)_{\lambda}}+\left(-C_{Z 4} u_{(j+1)_{\lambda}}+C_{Z 2}\right) \zeta_{(j+1)_{\lambda}} \\
& +C_{\mathrm{Z8}}\left(\theta_{(j+1)_{\lambda}}-\theta_{(j-1)_{\lambda}}\right) \\
& -C_{Z 7}\left(\theta_{(i+1)_{\lambda}}-\theta_{(i-1)_{\lambda}}\right)
\end{aligned}
$$

Implícito en $Y$

$$
\begin{aligned}
\left(-C_{Z 4} u_{(j-1)_{\lambda}}-C_{Z 5}\right) \zeta_{(j-1)_{\lambda}}^{k+1}+C_{Z 9} \zeta_{(j)_{\lambda}}^{k+1}+\left(C_{Z 4} v_{(j+1)_{\lambda}}-C_{Z 5}\right) \zeta_{(j+1)_{\lambda}}^{k+1} & =\left(C_{Z 1} u_{(i-1)_{\lambda}}+C_{Z 2}\right) \zeta_{(i-1)_{\lambda}}^{*} \\
+C_{Z 10} \zeta_{(i)_{\lambda}}^{*} & +\left(-C_{Z 1} u_{(i+1)_{\lambda}}+C_{Z 2}\right) \zeta_{(i+1)_{\lambda}}^{*} \\
& +C_{Z 8}\left(\theta_{(j+1)_{\lambda}}-\theta_{(j-1)_{\lambda}}\right) \\
& -C_{Z 7}\left(\theta_{(i+1)_{\lambda}}-\theta_{(i-1)_{\lambda}}\right)
\end{aligned}
$$

Donde:

$$
\begin{aligned}
C_{Z 1} & =\frac{\alpha_{\zeta}}{2 \operatorname{Pr} \Delta X} \\
C_{Z 2} & =\frac{\alpha_{\zeta}}{2 \Delta X^{2}} \\
C_{Z 3} & =\frac{2}{\Delta t}+2 C_{Z 2} \\
C_{Z 4} & =\frac{\alpha_{\zeta}}{2 \operatorname{Pr} \Delta Y} \\
C_{Z 5} & =\frac{\alpha_{\zeta}}{\Delta Y^{2}} \\
C_{Z 6} & =\frac{2}{\Delta t}-2 C_{Z 5} \\
C_{Z 7} & =\frac{\alpha_{\zeta} \operatorname{Ra}[\sin (\varphi)]}{2 \Delta X}
\end{aligned}
$$




$$
\begin{aligned}
& C_{Z 8}=\frac{\alpha_{\zeta} R a[\cos (\varphi)]}{2 \Delta Y} \\
& C_{Z 9}=\frac{2}{\Delta t}+2 C_{Z 5} \\
& C_{Z 10}=\frac{2}{\Delta t}-2 C_{Z 2}
\end{aligned}
$$

El método de solución de (C-64) y (C-65) es el mismo al utilizarlo para la ecuación de energía

\section{DISCRETIZACIÓN DE LA ECUACIÓN DE FUNCIÓN DE CORRIENTE}

$$
\frac{1}{\alpha_{\Psi}} \frac{\partial \Psi_{\lambda}}{\partial t}=\frac{\partial^{2} \Psi_{\lambda}}{\partial X^{2}}+\frac{\partial^{2} \Psi_{\lambda}}{\partial Y^{2}}+\zeta_{\lambda}
$$

En la ecuación (C-76) no existen términos convectivos para $\Psi$ ni tampoco existen coeficientes dependientes. Las dos ecuaciones resultantes al aplicar la adimensionalización son:

Implícito en $X$

$$
-C_{P 1} \Psi_{(i-1)_{\lambda}}^{*}+C_{P 2} \Psi_{i}^{*}-C_{P 1} \Psi_{(i+1)_{\lambda}}^{*}=C_{P 3} \Psi_{(j-1)_{\lambda}}+C_{P 4} \Psi_{(j)_{\lambda}}+C_{P 3} \Psi_{(j+1)_{\lambda}}+\alpha_{\Psi} \zeta_{i, j}
$$

Implícito en $Y$

$$
-C_{P 3} \Psi_{(j-1)_{\lambda}}^{k+1}+C_{P 5} \Psi_{i}^{k+1}-C_{P 3} \Psi_{(j+1)_{\lambda}}^{k+1}=C_{P 1} \Psi_{(i-1)_{\lambda}}^{*}+C_{P 6} \Psi_{(i)_{\lambda}}^{*}+C_{P 1} \Psi_{(i+1)_{\lambda}}^{*}+\alpha_{\Psi} \zeta_{i, j}
$$

Donde:

$$
\begin{aligned}
& C_{P 1}=\frac{\alpha_{\Psi}}{\Delta X^{2}} \\
& C_{P 2}=\frac{2}{\Delta t}-2 C_{P 1} \\
& C_{P 3}=\frac{\alpha_{\Psi}}{\Delta Y^{2}} \\
& C_{P 4}=\frac{2}{\Delta t}-2 C_{P 3} \\
& C_{P 5}=\frac{2}{\Delta t}+2 C_{P 3} \\
& C_{P 6}=\frac{2}{\Delta t}-2 C_{P 1}
\end{aligned}
$$


Para las condiciones interregionales utilizando las condiciones de Beavers y Joseph se tiene la siguiente expresión:

$$
\zeta_{N X I, j}=-\alpha\left(v_{\eta}-v_{\omega}\right)-\left(\frac{\partial u}{\partial y}\right)_{\eta}
$$

Sustituyendo la definición de las componentes de la velocidad en función del potencial

$$
\frac{\partial}{\partial X}\left(\frac{\partial \Psi}{\partial X}\right)_{\eta}=\alpha\left[\left(\frac{\partial \Psi}{\partial X}\right)_{\eta}-\left(\frac{\partial \Psi}{\partial X}\right)_{\omega}\right]
$$

Aplicando discretización hacia delante en la región porosa y hacia atrás en la región fluida de acuerdo a los espaciamientos mostrados en la Figura C.3 se tiene la siguiente expresión para la función de corriente:

$$
\begin{aligned}
\Psi_{N X I, j}= & \frac{\alpha \Delta X\left(5 \Psi_{N X I-1}-4 \Psi_{N X I-2}+\Psi_{N X I-3}\right)}{4} \\
& {[\underbrace{\left(3 \Psi_{N X I-1, j}-4 \Psi_{N X I-2, j}+\Psi_{N X I-3, j}\right)}_{\text {Región fluida }}-\underbrace{\left(-\Psi_{N X I+3, j}+4 \Psi_{N X I+2, j}-3 \Psi_{N X I+1, j}\right)}_{\text {Región porosa }}] }
\end{aligned}
$$

Sustituyendo las definiciones de las componentes de la velocidad y de la derivada expresada por la ecuación (C-12) se tiene la condición interregional para la vorticidad

$$
\zeta_{N X I, j}=-\alpha[\underbrace{\left(-\frac{\partial \Psi}{\partial X}\right)_{\eta}}_{\text {Hacia atrás }}-\underbrace{\left(-\frac{\partial \Psi}{\partial X}\right)_{\omega}}_{\text {Hacia adelante }}]-\underbrace{\frac{\partial}{\partial Y}\left(\frac{\partial \Psi}{\partial Y}\right)_{\eta}}_{\text {Hacia atrás }}
$$

Aplicando diferenciación hacia adelante en la región porosa y hacia atrás en la región fluida se tiene la expresión discretizada utilizada en la condición interregional:

$$
\begin{aligned}
\zeta_{N X I, j}= & \frac{\alpha}{2 \Delta X}\left(\frac{2 \Psi_{N X I-1, j}-5 \Psi_{N X I-2, j}+4 \Psi_{N X I-3, j}-\Psi_{N X I-4, j}}{(\Delta X)^{2}}\right)+ \\
& {[\underbrace{\left(3 \Psi_{N X I-1, j}-4 \Psi_{N X I-2, j}+\Psi_{N X I-3, j}\right)}_{\text {Región fluida }}-\underbrace{\left(-\Psi_{N X I+3, j}+4 \Psi_{N X I+2, j}-3 \Psi_{N X I+1, j}\right)}_{\text {Región porosa }}] }
\end{aligned}
$$




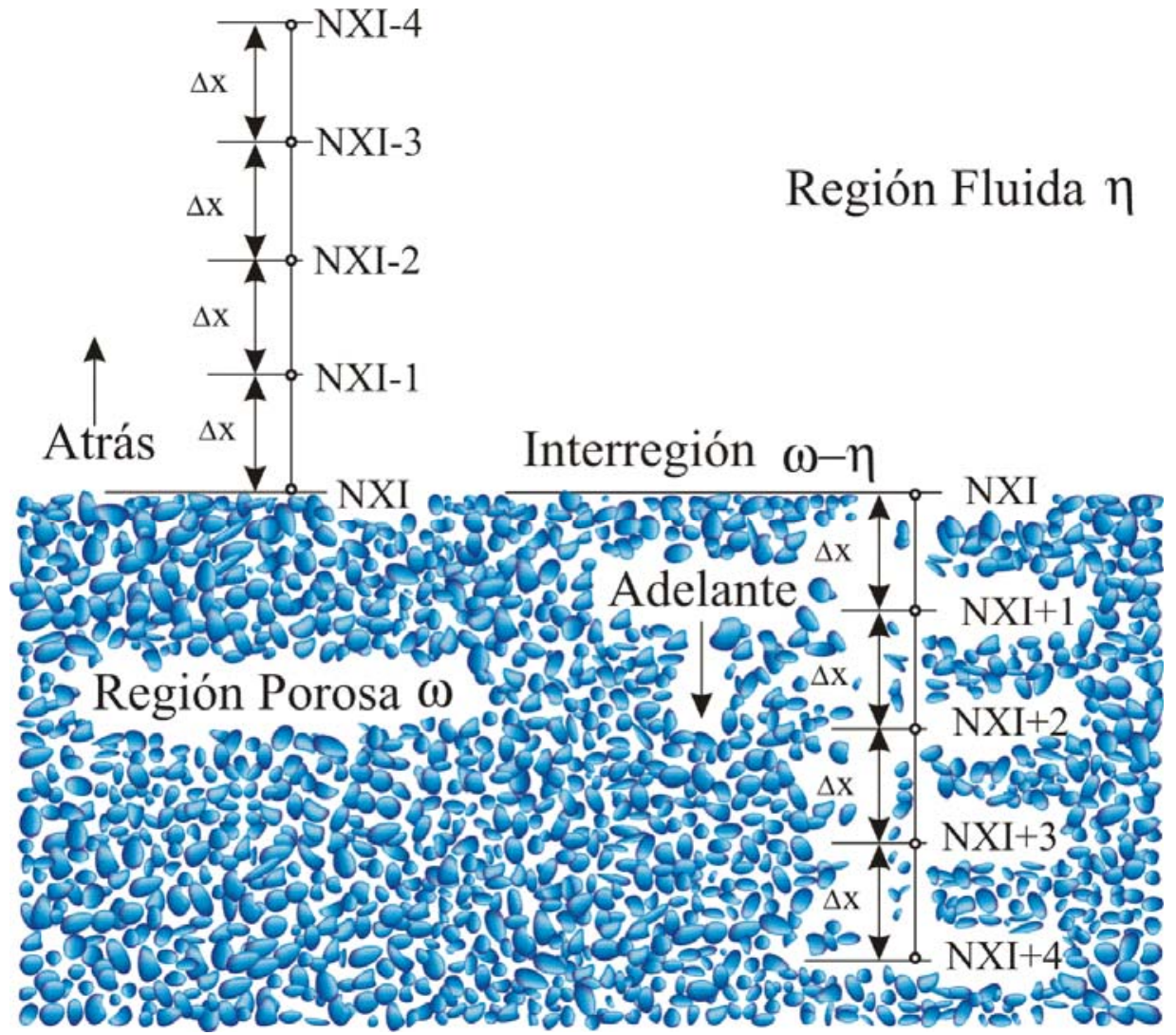

Figura C-3 Espaciamiento en la interregión para la discretización de la condición de Beavers y Joseph 


\section{Apéndice D}

\section{Discretización en diferencias finitas con espaciamiento variable.}

En el Capítulo 3 se presentaron las formas discretizadas de las ecuaciones en diferencias finitas, en ella se utilizaron diferencias centradas, hacia delante y hacia atrás para las primera y segunda derivada, parte importante de las del método numérico radica en la utilización de espaciamientos variables. A continuación se presentan el desarrollo para de las formas discretizadas de las derivadas, en la Figura D.1 se muestra la notación para los incrementos así como los subíndices utilizados:

\section{D.1 Diferenciación centrada.}

Las diferencias centradas se utilizan a partir del segundo nodo y hasta el penúltimo nodo en cada dirección

\section{D.1.1 Primer derivada.}

Para las diferencias centradas se comienza expandiendo en series de Taylor para los puntos alrededor del nodo $i$ en la dirección correspondiente.

$$
\begin{aligned}
& \xi_{i+1}=\xi_{i}+\Delta x_{i} \frac{\partial \xi_{i}}{\partial x}+\frac{\left(\Delta x_{i}\right)^{2}}{2 !} \frac{\partial^{2} \xi_{i}}{\partial x^{2}}+\frac{\left(\Delta x_{i}\right)^{3}}{3 !} \frac{\partial^{3} \xi_{i}}{\partial x^{3}}+\frac{\left(\Delta x_{i}\right)^{4}}{4 !} \frac{\partial^{4} \xi_{i}}{\partial x^{4}}+\ldots \\
& \xi_{i-1}=\xi_{i}-\Delta x_{i-1} \frac{\partial \xi_{i}}{\partial x}+\frac{\left(\Delta x_{i-1}\right)^{2}}{2 !} \frac{\partial^{2} \xi_{i}}{\partial x^{2}}-\frac{\left(\Delta x_{i-1}\right)^{3}}{3 !} \frac{\partial^{3} \xi_{i}}{\partial x^{3}}+\frac{\left(\Delta x_{i-1}\right)^{4}}{4 !} \frac{\partial^{4} \xi_{i}}{\partial x^{4}}+\ldots
\end{aligned}
$$



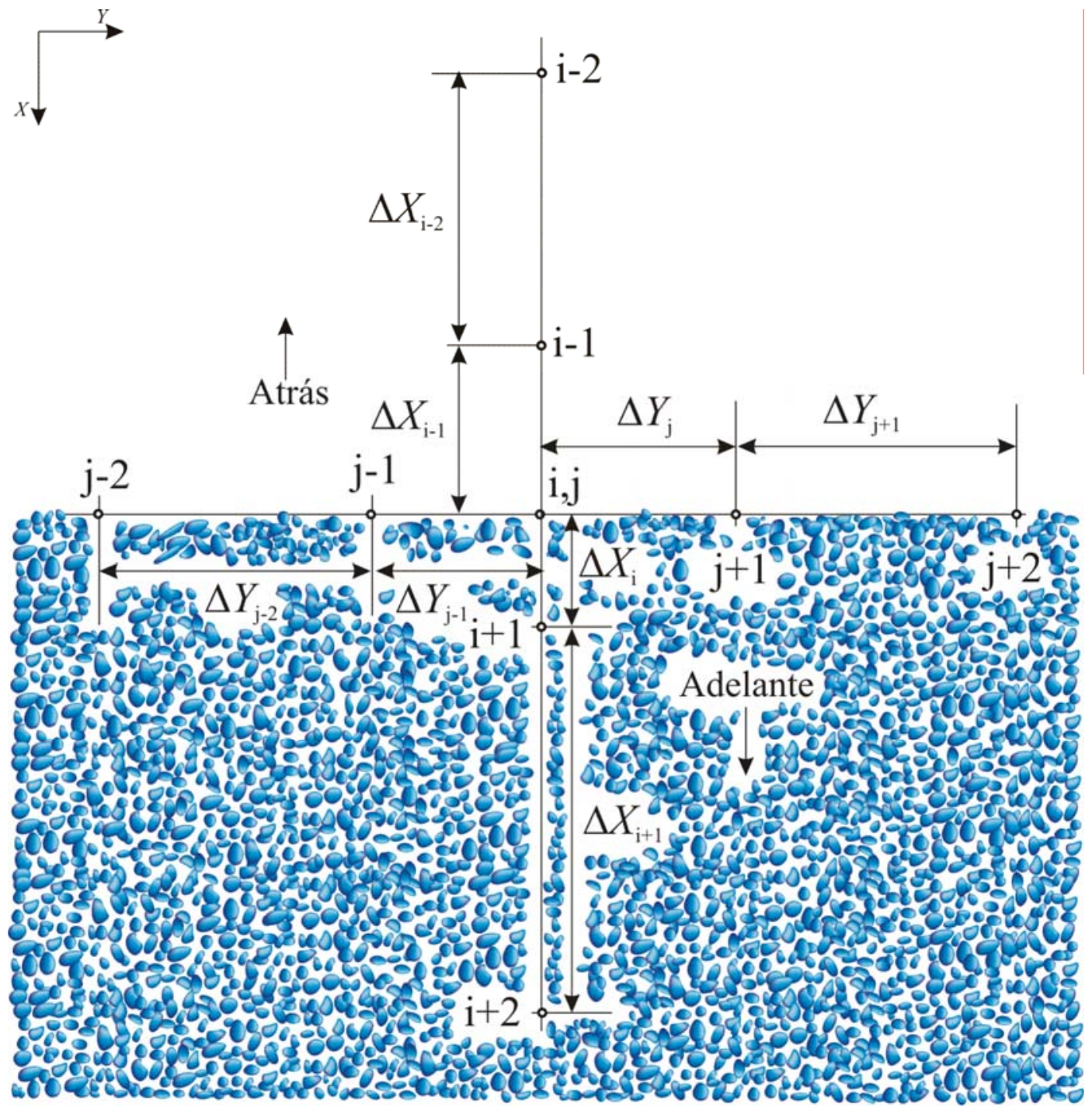

Figura D.1.- Celda computacional utilizada para la discretización de las ecuaciones diferenciales en diferencias finitas. 
Realizando la operación: (D-1)* $\left(\Delta x_{i-1}\right)^{2}-(\mathrm{D}-2) *\left(\Delta x_{i}\right)^{2}$

$$
\begin{aligned}
\left(\Delta x_{i-1}\right)^{2} \xi_{i+1}-\left(\Delta x_{i}\right)^{2} \xi_{i-1}= & \left(\Delta x_{i-1}\right)^{2} \xi_{i}-\left(\Delta x_{i}\right)^{2} \xi_{i}+\Delta x_{i}\left(\Delta x_{i-1}\right)^{2} \frac{\partial \xi_{i}}{\partial x}+\Delta x_{i-1}\left(\Delta x_{i}\right)^{2} \frac{\partial \xi_{i}}{\partial x}+ \\
& \frac{1}{3 !}\left[\left(\Delta x_{i}\right)^{3}\left(\Delta x_{i-1}\right)^{2}+\left(\Delta x_{i-1}\right)^{3}\left(\Delta x_{i}\right)^{2}\right] \frac{\partial^{3} \xi}{\partial x^{3}}
\end{aligned}
$$

Despejando la primer derivada de (D-3)

$$
\begin{aligned}
\frac{\partial \xi}{\partial x}= & \frac{\Delta x_{i-1}}{\Delta x_{i}\left(\Delta x_{i-1}+\Delta x_{i}\right)} \xi_{i+1}+\frac{\left(\Delta x_{i}\right)^{2}-\left(\Delta x_{i-1}\right)^{2}}{\Delta x_{i} \Delta x_{i-1}\left(\Delta x_{i-1}+\Delta x_{i}\right)} \xi_{i}-\frac{\Delta x_{i}}{\Delta x_{i-1}\left(\Delta x_{i-1}+\Delta x_{i}\right)} \xi_{i-1}+ \\
& \sim \underbrace{\left[\frac{\left(\Delta x_{i}\right)^{2}\left(\Delta x_{i-1}\right)^{2}\left(\Delta x_{i}+\Delta x_{i-1}\right)}{\Delta x_{i} \Delta x_{i-1}\left(\Delta x_{i}+\Delta x_{i-1}\right)}\right]}_{\left[\Delta x_{i} \Delta x_{i-1}\right]}
\end{aligned}
$$

Reagrupando términos se tiene la expresión para la primer derivada:

$$
\left.\frac{\partial \xi}{\partial x}\right|_{i}=C_{X 1} \xi_{i-1}+C_{X 2} \xi_{i}+C_{X 3} \xi_{i+1}
$$

Donde:

$$
\begin{aligned}
& C_{X 1}=-\frac{\left(\Delta x_{i}\right)^{2}}{\Delta x_{i}\left(\Delta x_{i-1}\right)^{2}+\Delta x_{i-1}\left(\Delta x_{i}\right)^{2}} \\
& C_{X 2}=\frac{\left(\Delta x_{i}\right)^{2}-\left(\Delta x_{i-1}\right)^{2}}{\Delta x_{i}\left(\Delta x_{i-1}\right)^{2}+\Delta x_{i-1}\left(\Delta x_{i}\right)^{2}} \\
& C_{X 3}=\frac{\left(\Delta x_{i-1}\right)^{2}}{\Delta x_{i}\left(\Delta x_{i-1}\right)^{2}+\Delta x_{i-1}\left(\Delta x_{i}\right)^{2}}
\end{aligned}
$$

\section{D.1.2 Segunda derivada.}

Realizando la operación: (D-1)*( $\left(\Delta x_{i-1}\right)+(\mathrm{D}-2) *\left(\Delta x_{i}\right)$ :

$$
\begin{gathered}
\left(\Delta x_{i-1}\right) \xi_{i+1}+\left(\Delta x_{i}\right) \xi_{i-1}=\left(\Delta x_{i-1}+\Delta x_{i}\right) \xi_{i}+\frac{1}{2 !}\left[\Delta x_{i-1}\left(\Delta x_{i}\right)^{2}+\Delta x_{i}\left(\Delta x_{i-1}\right)^{2}\right] \frac{\partial^{2} \xi_{i}}{\partial x^{2}}+ \\
\frac{1}{3 !}\left[\Delta x_{i-1}\left(\Delta x_{i}\right)^{3}-\Delta x_{i}\left(\Delta x_{i-1}\right)^{3}\right] \frac{\partial^{3} \xi}{\partial x^{3}}
\end{gathered}
$$


Despejando la segunda derivada de la ecuación (D-9)

$$
\begin{gathered}
\frac{\partial^{2} \xi}{\partial x^{2}}=\frac{2 \Delta x_{i-1}}{\left[\Delta x_{i-1} \Delta x_{i}\left(\Delta x_{i-1}+\Delta x_{i}\right)\right]} \xi_{i+1}-\frac{2\left(\Delta x_{i-1}+\Delta x_{i}\right)}{\left[\Delta x_{i-1} \Delta x_{i}\left(\Delta x_{i-1}+\Delta x_{i}\right)\right]} \xi_{i}+ \\
\frac{2 \Delta x_{i}}{\left[\Delta x_{i-1} \Delta x_{i}\left(\Delta x_{i-1}+\Delta x_{i}\right)\right]} \xi_{i-1}+\underset{\sim}{O} \underbrace{\left[\frac{\Delta x_{i-1} \Delta x_{i}\left(\Delta x_{i}^{2}-\Delta x_{i-1}^{2}\right)}{\Delta x_{i-1} \Delta x_{i}\left(\Delta x_{i}-\Delta x_{i-1}\right)}\right]}_{\left[\Delta x_{i}+\Delta x_{i-1}\right]}
\end{gathered}
$$

Reagrupando términos se tiene la expresión para la segunda derivada:

$$
\begin{aligned}
& \left.\frac{\partial^{2} \xi}{\partial x^{2}}\right|_{i}=C_{X 4} \xi_{i-1}+C_{X 5} \xi_{i}+C_{X 6} \xi_{i+1} \\
& C_{X 4}=\frac{2}{\Delta x_{i-1}\left(\Delta x_{i-1}+\Delta x_{i}\right)} \\
& C_{X 5}=-\frac{2}{\Delta x_{i-1} \Delta x_{i}} \\
& C_{X 6}=-\frac{2}{\Delta x_{i}\left(\Delta x_{i-1}+\Delta x_{i}\right)}
\end{aligned}
$$

\section{D.2 Diferenciación hacia adelante.}

Las diferencias hacia delante se utilizan en las paredes cuyo sentido del eje es el mismo a la dirección de la discretización y en la interregión para el medio poroso (ver Figura D.1)

\section{D.2.1 Primer derivada.}

Para las derivadas hacia delante se realiza una expansión en series de Taylor para los nodos $i+1$ e $i+2$ obteniéndose las siguientes expresiones:

$$
\begin{aligned}
\xi_{i+1}= & \xi_{i}+\Delta x_{i} \frac{\partial \xi_{i}}{\partial x}+\frac{\left(\Delta x_{i}\right)^{2}}{2 !} \frac{\partial^{2} \xi_{i}}{\partial x^{2}}+\frac{\left(\Delta x_{i}\right)^{3}}{3 !} \frac{\partial^{3} \xi_{i}}{\partial x^{3}}+\frac{\left(\Delta x_{i}\right)^{4}}{4 !} \frac{\partial^{4} \xi_{i}}{\partial x^{4}}+\ldots \\
\xi_{i+2}= & \xi_{i}+\left(\Delta x_{i}+\Delta x_{i+1}\right) \frac{\partial \xi_{i}}{\partial x}+\frac{\left(\Delta x_{i}+\Delta x_{i+1}\right)^{2}}{2 !} \frac{\partial^{2} \xi_{i}}{\partial x^{2}}+\frac{\left(\Delta x_{i}+\Delta x_{i+1}\right)^{3}}{3 !} \frac{\partial^{3} \xi_{i}}{\partial x^{3}}+ \\
& \frac{\left(\Delta x_{i}+\Delta x_{i+1}\right)^{4}}{4 !} \frac{\partial^{4} \xi_{i}}{\partial x^{4}}+\ldots
\end{aligned}
$$


Realizando la operación: (D-15)* $\left(\Delta x_{i}+\Delta x_{i+1}\right)^{2}-(\mathrm{C}-16)$ por $\left(\Delta x_{i}\right)^{2}$ y despejando la primer derivada se tiene:

$$
\begin{gathered}
\left.\frac{\partial \xi_{i}}{\partial x}\right|_{i}=\frac{\Delta x_{i}\left(\Delta x_{i}+2 \Delta x_{i+1}\right) \xi_{i}-\left(\Delta x_{i}+\Delta x_{i+1}\right)^{2} \xi_{i+1}+\left(\Delta x_{i}\right)^{2} \xi_{i+2}}{-\Delta x_{i} \Delta x_{i+1}\left(\Delta x_{i}+\Delta x_{i+1}\right)}+ \\
\underset{\sim}{O}\left[\frac{\left\{\left(\Delta x_{i+1}\right)^{3}\left(\Delta x_{i}+\Delta x_{i+1}\right)-\left(\Delta x_{i}\right)^{2}\left(\Delta x_{i}+\Delta x_{i+1}\right)^{2}\right\}}{\Delta x_{i} \Delta x_{i+1}}\right]
\end{gathered}
$$

Reagrupando (D-17):

$$
\begin{aligned}
& \frac{\left.\partial \xi_{i}\right|_{\partial x}=C F_{X_{1}} \xi_{i}+C F_{X_{2}} \xi_{i+1}+C F_{X_{3}} \xi_{i+2}}{C F_{X_{1}}=-\frac{\Delta x_{i}+2 \Delta x_{i+1}}{\Delta x_{i+1}\left(\Delta x_{i}+\Delta x_{i+1}\right)}} \\
& C F_{X_{2}}=\frac{\Delta x_{i}+\Delta x_{i+1}}{\Delta x_{i} \Delta x_{i+1}} \\
& C F_{X_{3}}=-\frac{\Delta x_{i+1}}{\Delta x_{i}\left(\Delta x_{i}+\Delta x_{i+1}\right)}
\end{aligned}
$$

\section{D.2.2 Segunda derivada.}

$$
\begin{gathered}
\text { Realizando: (D-15)*( }\left(\Delta x_{i}+\Delta x_{i+1}\right)-(\mathrm{D}-16) *\left(\Delta x_{i}\right) \\
\left(\Delta x_{i}+\Delta x_{i+1}\right) \xi_{i+1}-\left(\Delta x_{i}\right) \xi_{i+2}=\Delta x_{i} \xi_{i}+\frac{1}{2 !}\left(-\Delta x_{i}^{2} \Delta x_{i+1}+\Delta x_{i+1}^{2}\right) \frac{\partial^{2} \xi_{i}}{\partial x^{2}}+ \\
\frac{1}{3 !}\left(-2 \Delta x_{i}^{3} \Delta x_{i+1}-3 \Delta x_{i}^{2} \Delta x_{i+1}-\Delta x_{i+1}^{4}\right) \frac{\partial^{3} \xi_{i}}{\partial x^{3}}
\end{gathered}
$$

Despejando la segunda derivada de la ecuación (D-22):

$$
\begin{gathered}
\left.\frac{\partial^{2} \xi_{i}}{\partial x^{2}}\right|_{i}=\frac{2\left(\Delta x_{i}+\Delta x_{i+1}\right) \xi_{i+1}+2 \Delta x_{i} \xi_{i+2}-2 \Delta x_{i+1} \xi_{i}}{\Delta x_{i+1}\left(\Delta x_{i+1}-\Delta x_{i}^{2}\right)}+ \\
\underset{\sim}{O}\left[\frac{2 \Delta x_{i}^{3} \Delta x_{i+1}+3 \Delta x_{i}^{2} \Delta x_{i+1}+\Delta x_{i+1}^{4}}{\Delta x_{i+1}\left(\Delta x_{i+1}-\Delta x_{i}^{2}\right)}\right]
\end{gathered}
$$


Reagrupando (D-23):

$$
\begin{aligned}
& \left.\frac{\partial^{2} \xi_{i}}{\partial x^{2}}\right|_{i}=C F_{X_{4}} \xi_{i}+C F_{X_{5}} \xi_{i+1}+C F_{X_{6}} \xi_{i+2} \\
& C F_{X_{4}}=-\frac{2}{\Delta x_{i+1}-\Delta x_{i}^{2}} \\
& C F_{X_{5}}=\frac{2\left(\Delta x_{i}+\Delta x_{i+1}\right)}{\Delta x_{i+1}\left(\Delta x_{i+1}-\Delta x_{i}^{2}\right)} \\
& C F_{X_{6}}=\frac{2 \Delta x_{i}}{\Delta x_{i+1}\left(\Delta x_{i+1}-\Delta x_{i}^{2}\right)}
\end{aligned}
$$

\section{D.3 Diferenciación hacia atrás.}

Las diferencias hacia atrás se utilizan en las paredes cuyo sentido del eje es el contrario a la dirección de la discretización y en la interregión para la región fluida (ver Figura D.1)

\section{D.3.1 Primer derivada.}

Para las derivadas hacia atrás se realiza una expansión en series de Taylor para los nodos i-1 e i-2 (ver Figura D-1), obteniéndose las siguientes expresiones:

$$
\begin{aligned}
\xi_{i-1}= & \xi_{i}-\Delta x_{i-1} \frac{\partial \xi_{i}}{\partial x}+\frac{\left(\Delta x_{i-1}\right)^{2}}{2 !} \frac{\partial^{2} \xi_{i}}{\partial x^{2}}-\frac{\left(\Delta x_{i-1}\right)^{3}}{3 !} \frac{\partial^{3} \xi_{i}}{\partial x^{3}}+\frac{\left(\Delta x_{i-1}\right)^{4}}{4 !} \frac{\partial^{4} \xi_{i}}{\partial x^{4}}+\ldots \\
\xi_{i-2}= & \xi_{i}-\left(\Delta x_{i-1}+\Delta x_{i-2}\right) \frac{\partial \xi_{i}}{\partial x}+\frac{\left(\Delta x_{i-1}+\Delta x_{i-2}\right)^{2}}{2 !} \frac{\partial^{2} \xi_{i}}{\partial x^{2}}-\frac{\left(\Delta x_{i-1}+\Delta x_{i-2}\right)^{3}}{3 !} \frac{\partial^{3} \xi_{i}}{\partial x^{3}}+ \\
& \frac{\left(\Delta x_{i-1}+\Delta x_{i-2}\right)^{4}}{4 !} \frac{\partial^{4} \xi_{i}}{\partial x^{4}}+\ldots
\end{aligned}
$$

Realizando la operación: (D-28)*( $\left(\Delta x_{i-1}+\Delta x_{i-2}\right)^{2}-(\mathrm{C}-16)^{*}\left(\Delta x_{i-1}\right)^{2}$ y despejando la primer derivada se tiene:

$$
\left.\frac{\partial \xi_{i}}{\partial x}\right|_{i}=\frac{\Delta x_{i-2}\left(\Delta x_{i-2}+2 \Delta x_{i-1}\right) \xi_{i}+\left(\Delta x_{i-1}\right)^{2} \xi_{i-1}-\left(\Delta x_{i-2}+\Delta x_{i-1}\right)^{2} \xi_{i-2}}{\Delta x_{i-2} \Delta x_{i-1}\left(\Delta x_{i-1}+\Delta x_{i-1}\right)}+
$$




$$
\underset{\sim}{\mathcal{O}}\left[\frac{\left(\Delta x_{i-1}\right)^{2}\left(\Delta x_{i-2}+\Delta x_{i-1}\right)\left(\Delta x_{i-2}\right)}{\Delta x_{i-2} \Delta x_{i-1}\left(\Delta x_{i-2}+\Delta x_{i-1}\right)}\right]
$$

Reagrupando (D-30):

$$
\begin{aligned}
\left.\frac{\partial \xi_{i}}{\partial x}\right|_{i} & =C B_{X_{1}} \xi_{i}+C B_{X_{2}} \xi_{i-1}+C B_{X_{3}} \xi_{i-2} \\
C B_{X_{1}} & =\frac{\Delta x_{i-2}+2 \Delta x_{i-1}}{\Delta x_{i-1}\left(\Delta x_{i-2}+\Delta x_{i-1}\right)} \\
C B_{X_{2}} & =-\frac{\Delta x_{i-2}+\Delta x_{i-1}}{\Delta x_{i-2} \Delta x_{i-1}} \\
C B_{X_{3}} & =\frac{\Delta x_{i-1}}{\Delta x_{i-2}\left(\Delta x_{i-2}+\Delta x_{i-1}\right)}
\end{aligned}
$$

\section{D.3.2 Segunda derivada.}

$$
\begin{gathered}
\text { Realizando (D-28)*( }\left(\Delta x_{i-1}+\Delta x_{i-2}\right)-(\mathrm{D}-29) *\left(\Delta x_{i-1}\right) \\
\begin{aligned}
&\left(\Delta x_{i-1}+\Delta x_{i-2}\right) \xi_{i-1}-\left(\Delta x_{i-1}\right) \xi_{i-2}=\Delta x_{i-2} \xi_{i}+ \\
& \frac{\left(\Delta x_{i-1}\right)^{2}\left(\Delta x_{i-1}+\Delta x_{i-2}\right)+\Delta x_{i-1}\left(\Delta x_{i-1}+\Delta x_{i-2}\right)^{2}}{2 !} \frac{\partial^{2} \xi_{i}}{\partial x^{2}}+ \\
& \frac{1}{3 !}\left(-2 \Delta x_{i}^{3} \Delta x_{i+1}-3 \Delta x_{i}^{2} \Delta x_{i+1}-\Delta x_{i+1}^{4}\right) \frac{\partial^{3} \xi_{i}}{\partial x^{3}}
\end{aligned}
\end{gathered}
$$

Despejando la segunda derivada de (D.42)

$$
\begin{gathered}
\left.\frac{\partial^{2} \xi_{i}}{\partial x^{2}}\right|_{i}=\frac{2\left[\left(\Delta x_{i-1}+\Delta x_{i-2}\right) \xi_{i-1}-\Delta x_{i-1} \xi_{i-1}-\Delta x_{i-2} \xi_{i}\right]}{\left(\Delta x_{i-1}\right)^{2}\left(\Delta x_{i-1}+\Delta x_{i-2}\right)+\left(\Delta x_{i-1}\right)\left(\Delta x_{i-1}+\Delta x_{i-2}\right)^{2}}+ \\
\underset{\sim}{ }\left[\frac{\left(\Delta x_{i-1}-\Delta x_{i-2}\right)^{2}-\left(\Delta x_{i-1}\right)^{2}}{2 \Delta x_{i-1}-\Delta x_{i-2}}\right]
\end{gathered}
$$

Reagrupando (D-43):

$$
\left.\frac{\partial^{2} \xi_{i}}{\partial x^{2}}\right|_{i}=C B_{X_{4}} \xi_{i}+C B_{X_{5}} \xi_{i-1}+C B_{X_{6}} \xi_{i-2}
$$




$$
\begin{aligned}
C B_{X_{4}} & =-\frac{2 \Delta x_{i-2}}{\Delta x_{i-1}\left(\Delta x_{i-1}+\Delta x_{i-2}\right)\left(2 \Delta x_{i-1}+\Delta x_{i-2}\right)} \\
C B_{X_{5}} & =\frac{2}{\Delta x_{i-1}\left(2 \Delta x_{i-1}+\Delta x_{i-2}\right)} \\
C B_{X_{6}} & =-\frac{2}{\left(\Delta x_{i-1}+\Delta x_{i-2}\right)\left(2 \Delta x_{i-1}+\Delta x_{i-2}\right)}
\end{aligned}
$$




\section{Aal}

Casa abierta al tiempo

UNIVERSIDAD AUTONOMA METROPOLITANA

Fecha : 14/07/2005

Página :

$1 / 1$

CONSTANCIA DE PRESENTACION DE EXAMEN DE GRADO La Universidad Autónoma Metropolitana extiende la presente CONSTANCIA DE
PRESENTACION DE EXAMEN DE GRADO de DOCTOR EN CIENCIAS del alumnO JOSE JAVIER VALENCIA LOPEZ, matrícula 96100833, quien cumplió con los 180 créditos correspondientes a las unidades de enseñanza aprendizaje del plan de estudio. Con fecha veintidós de julio del 2005 presentó la DEFENSA de su DISERTACIÓN PÚBLICA cuya denominación es:

ESTUDIO DE LA TRANSFERENCIA DE ENERGIA, MASA Y CANTIDAD DE MOVIMIENTO EN SISTEMAS FLUIDO-MEDIO POROSO.

Cabe mencionar que la aprobación de la Disertación pública tiene un valor de 180 créditos y el programa consta de 360 créditos.

El jurado del examen ha tenido a bien otorgarle la calificación de:

Aprobar

JURADO

Presidente

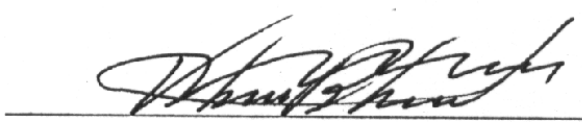

DRA. ROSA MARIA VELASCO BELMONT

Vocal

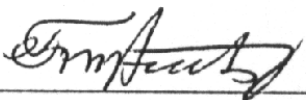

DR. FRANCISCO JAVIER SANCHEZ BERNABE

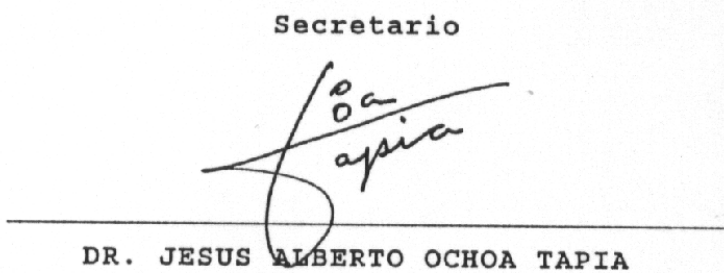

DR. JESUS ALBERTO OCHOA TAPIA

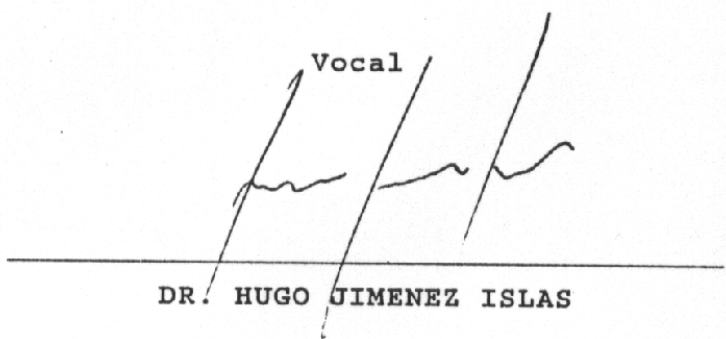

Vocal

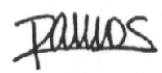

DR. EDUARDO RAMOS MORA

\section{UNIDAD IZTAPALAPA}

Coordinación de Sistemas Escolares

San Bafael Atlixco 186 Col Vicentina México D E 09340 Ando Poctal 555-300-00no 